\title{
Global Mirrors and Discrepant Transformations for Toric Deligne-Mumford Stacks
}

\author{
Hiroshi IRITANI
}

Department of Mathematics, Graduate School of Science, Kyoto University, Kitashirakawa-Oiwake-cho, Sakyo-ku, Kyoto, 606-8502, Japan

E-mail: iritani@math.kyoto-u.ac.jp

URL: https://www.math.kyoto-u.ac.jp/ iritani/

Received June 13, 2019, in final form March 29, 2020; Published online April 22, 2020 https://doi.org/10.3842/SIGMA.2020.032

\begin{abstract}
We introduce a global Landau-Ginzburg model which is mirror to several toric Deligne-Mumford stacks and describe the change of the Gromov-Witten theories under discrepant transformations. We prove a formal decomposition of the quantum cohomology D-modules (and of the all-genus Gromov-Witten potentials) under a discrepant toric wall-crossing. In the case of weighted blowups of weak-Fano compact toric stacks along toric centres, we show that an analytic lift of the formal decomposition corresponds, via the $\widehat{\Gamma}$-integral structure, to an Orlov-type semiorthogonal decomposition of topological $K$ groups. We state a conjectural functoriality of Gromov-Witten theories under discrepant transformations in terms of a Riemann-Hilbert problem.
\end{abstract}

Key words: quantum cohomology; mirror symmetry; toric variety; Landau-Ginzburg model; Gamma-integral structure

2020 Mathematics Subject Classification: 14N35; 14J33; 53D45

\section{Contents}

1 Introduction $\quad 3$

1.1 Quantum cohomology D-modules . . . . . . . . . . . . . . . . . . . 3

1.2 Global Landau-Ginzburg models and toric wall-crossings . . . . . . . . . . . . . 4

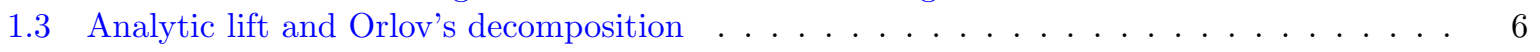

1.4 Related works . . . . . . . . . . . . . . . . . . . . . 7

2 Preliminaries $\quad 8$

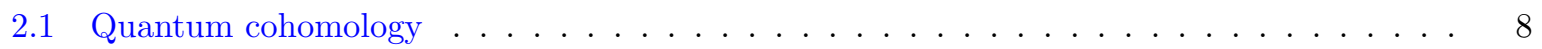

2.2 Kähler moduli space . . . . . . . . . . . . . . . . . . . . . . . . . 10

2.3 Quantum D-module . . . . . . . . . . . . . . . . . . . . . . . . . 12

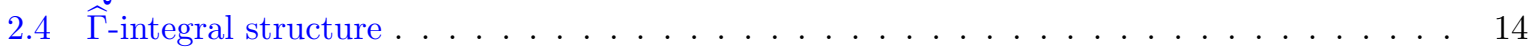

3 Global Landau-Ginzburg mirrors of toric DM stacks $\quad 16$

3.1 Toric data . . . . . . . . . . . . . . . . . . . . . . 16

3.1 .1 Toric DM stacks . . . . . . . . . . . . . . . . . . . . 17

3.1 .2 Picard group and second $($ co)homology . . . . . . . . . . . . . . . . 18

3.1 .3 Orbifold cohomology . . . . . . . . . . . . . . . . . . . . 18

3.2 Landau-Ginzburg model . . . . . . . . . . . . . . . . . . . . . . . . . . . . . . 19

3.3 Extended refined fan sequence and extended Mori cone . . . . . . . . . . . . . . . . 21

3.4 Local charts of the LG model . . . . . . . . . . . . . . . . . . . . . . . 23

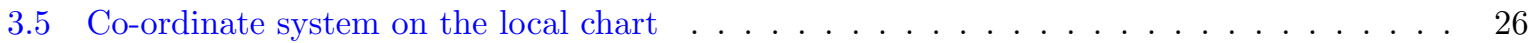

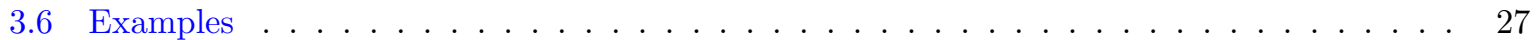

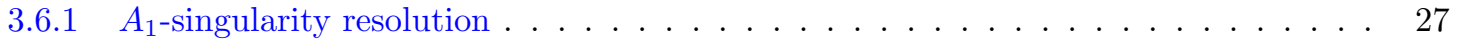

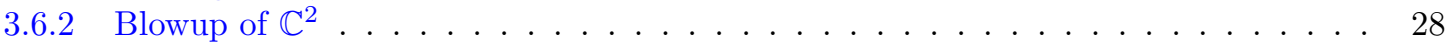

This paper is a contribution to the Special Issue on Integrability, Geometry, Moduli in honor of Motohico Mulase for his 65 th birthday. The full collection is available at https://www.emis.de/journals/SIGMA/Mulase.html 


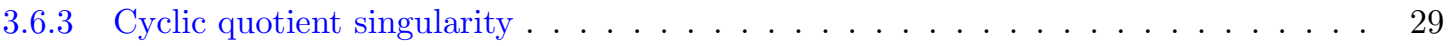

4 Mirror symmetry $\mathbf{3 0}$

4.1 Brieskorn module . . . . . . . . . . . . . . . . . . . . 30

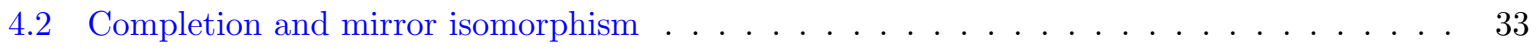

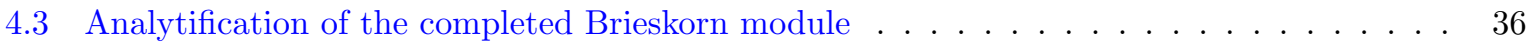

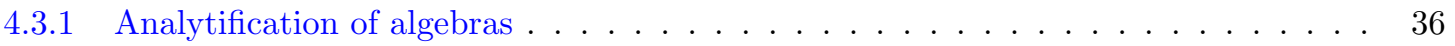

4.3 .2 Analytification of D-modules . . . . . . . . . . . . . . . . 38

4.3.3 The higher residue pairing on the analytified Brieskorn module . . . . . . . . . 41

4.4 Analytic mirror isomorphism . . . . . . . . . . . . . . . . . . . 43

5 Discrepant wall-crossings $\quad \mathbf{4 4}$

5.1 Discrepant transformation of smooth toric DM stacks . . . . . . . . . . . . . . 44

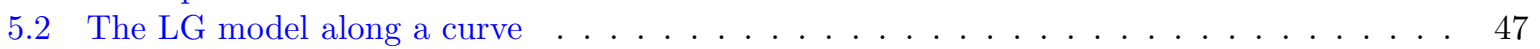

5.3 Decomposition of the Brieskorn module . . . . . . . . . . . . . . . . . . . . 49

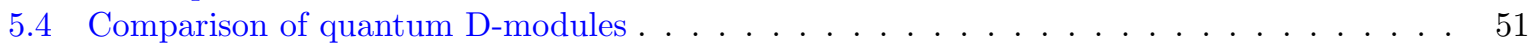

5.5 Comparison of Gromov-Witten theories in all genera . . . . . . . . . . . . . . . . . 52

5.5 .1 Ancestor potentials . . . . . . . . . . . . . . . . . . . . 52

5.5 .2 Givental's quantization formalism . . . . . . . . . . . . . . 53

5.5 .3 A relationship between ancestor potentials . . . . . . . . . . . . . . 54

5.5 .4 Givental's formula and the proof of Theorem $5.19 \ldots \ldots \ldots \ldots$

6 Formal decomposition and analytic lift $\quad \mathbf{5 6}$

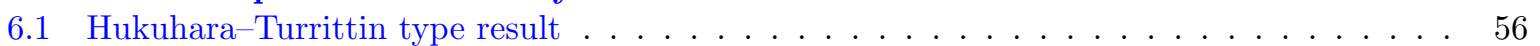

6.2 Asymptotic basis and marked reflection system . . . . . . . . . . . . . . . . . 59

6.3 Sectorial decomposition of the Brieskorn module . . . . . . . . . . . . . . . . . . 61

6.3.1 Formal decomposition of the Brieskorn module . . . . . . . . . . . . . . . 61

6.3.2 Brieskorn module over the small quantum cohomology locus . . . . . . . . . . . . . 62

6.3 .3 Analytic lift of the formal decomposition . . . . . . . . . . . . . . . . 64

7 Functoriality under toric birational morphisms $\quad 66$

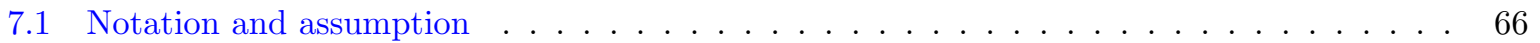

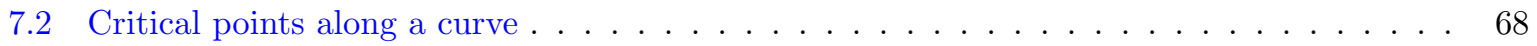

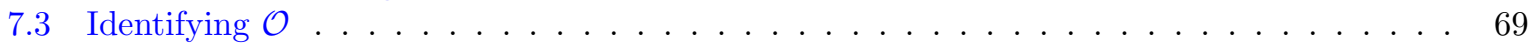

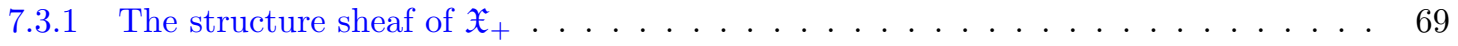

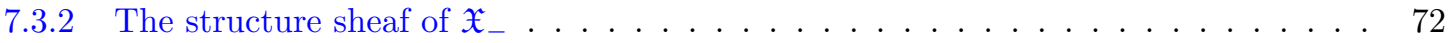

7.4 Inclusion of the local systems of Lefschetz thimbles . . . . . . . . . . . . . . . . 74

7.4.1 Convergent and divergent critical branches . . . . . . . . . . . . . . 74

7.4 .2 Local co-ordinate system around $0_{-}=0_{\boldsymbol{\Sigma}_{-}} \ldots \ldots \ldots \ldots \ldots \ldots$

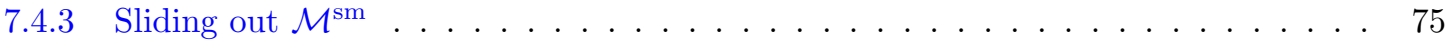

7.4.4 Kouchnirenko's condition and a local system of Lefschetz thimbles . . . . . . . 76

7.4.5 Inclusion of the local systems: statement and proof . . . . . . . . . . . . . 79

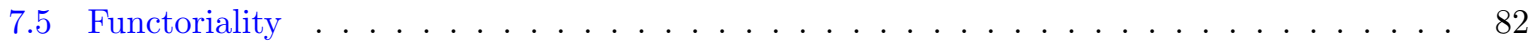

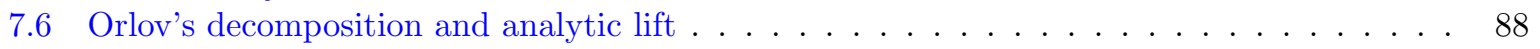

7.6.1 Decomposition of the relative homology mirror to Orlov's decomposition . . . . . . 88

7.6.2 Orlov's decomposition as a sectorial decomposition of the quantum D-module . . . 91

8 Conjecture and discussion $\quad \mathbf{9 6}$

8.1 General conjecture . . . . . . . . . . . . . . . . . . . . . 96

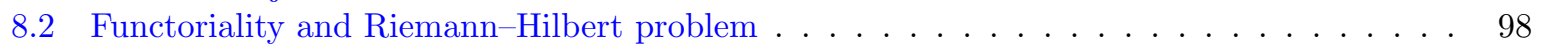

A The Brieskorn module in the weak Fano case 101

A.1 Generators and relations . . . . . . . . . . . . . . . . . . . . . 102

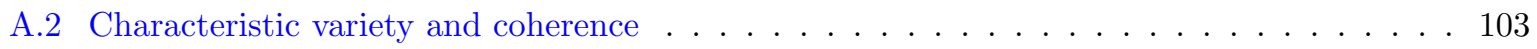

A.3 Locally freeness and $r a n k \ldots \ldots \ldots 4 \ldots \ldots$

B Proof of Lemma 7.24 105

$\begin{array}{ll}\text { References } & 107\end{array}$ 


\section{Introduction}

It is a very interesting problem to study how Gromov-Witten invariants (or quantum cohomology) change under birational transformations. When the birational transformation is crepant (or a K-equivalence), a conjecture of Yongbin Ruan [92] says that the quantum cohomology of $K$-equivalent spaces should be related to each other by analytic continuation in quantum parameters (see, e.g., [20, 30, 32, 77, 80]). In this paper, we are concerned with discrepant transformations, or more precisely, birational maps $\varphi: \mathfrak{X}_{+}-\rightarrow \mathfrak{X}_{-}$between smooth DeligneMumford stacks such that there exist projective birational morphisms $f_{ \pm}: \widehat{\mathfrak{X}} \rightarrow \mathfrak{X}_{ \pm}$satisfying $f_{-}=\varphi \circ f_{+}$and that $f_{+}^{*} K_{\mathfrak{X}_{+}}-f_{-}^{*} K_{\mathfrak{X}_{-}}$is a non-zero effective divisor. In this case, we write " $K_{\mathfrak{X}_{+}}>K_{\mathfrak{X}_{-}}$" by a slight abuse of notation

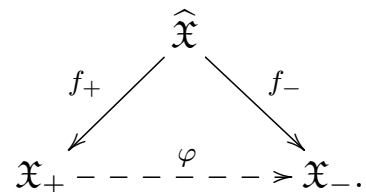

This includes the case where $\varphi$ is a blowup along a smooth subvariey. In this case, we do not expect that the quantum cohomology of $\mathfrak{X}_{+}$and $\mathfrak{X}_{-}$are related by analytic continuation because their ranks are different. Instead, we expect that the quantum cohomology of $\mathfrak{X}_{+}$would contain the quantum cohomology of $\mathfrak{X}_{-}$as a direct summand after analytic continuation. This is analogous to the conjecture that $D^{b}\left(\mathfrak{X}_{+}\right)$contains $D^{b}\left(\mathfrak{X}_{-}\right)$as a semiorthogonal summand $[9,13,71,72]$, where $D^{b}\left(\mathfrak{X}_{ \pm}\right)$denotes the derived category of coherent sheaves on $\mathfrak{X}_{ \pm}$. In this paper, we describe a decomposition of quantum cohomology D-modules (and of all genus Gromov-Witten potentials) for toric Deligne-Mumford stacks under discrepant transformations. Moreover, we show in special cases that the decomposition of quantum cohomology D-modules is induced by a semiorthogonal decomposition of derived categories (or more precisely of topological $K$-groups) via the $\widehat{\Gamma}$-integral structure $[62,70]$. We also formulate a general conjecture in view of our results in the toric case.

\subsection{Quantum cohomology D-modules}

Our central objects of study are quantum (cohomology) D-modules. Let $\mathfrak{X}$ be a smooth DeligneMumford stack. The genus-zero Gromov-Witten invariants define a family of (super)commutative product structures $\star_{\tau}$ on the orbifold cohomology group $H_{\mathrm{CR}}^{*}(\mathfrak{X})$ parametrized by $\tau \in$ $H_{\mathrm{CR}}^{*}(\mathfrak{X})$; this is called quantum cohomology. The product $\star_{\tau}$ then defines a meromorphic flat connection $\nabla$ called the quantum connection (or Dubrovin connection) on the trivial $H_{\mathrm{CR}}^{*}(\mathfrak{X})$ bundle over $H_{\mathrm{CR}}^{*}(\mathfrak{X}) \times \mathbb{C}$. It is given by the formulae

$$
\begin{aligned}
& \nabla_{\frac{\partial}{\partial \tau^{i}}}=\frac{\partial}{\partial \tau^{i}}+\frac{1}{z} \phi_{i \star_{\tau}}, \\
& \nabla_{z \frac{\partial}{\partial z}}=z \frac{\partial}{\partial z}-\frac{1}{z} E \star_{\tau}+\mu,
\end{aligned}
$$

where $(\tau, z)$ represents a point on the base $H_{\mathrm{CR}}^{*}(\mathfrak{X}) \times \mathbb{C},\left\{\tau^{i}\right\}$ are linear co-ordinates on $H_{\mathrm{CR}}^{*}(\mathfrak{X})$ dual to a basis $\left\{\phi_{i}\right\}, E$ is the so-called Euler vector field, and $\mu$ is the (constant) grading operator. This connection is self-dual with respect to the pairing $P$ between the fibres at $(\tau,-z)$ and $(\tau, z)$ induced by the orbifold Poincaré pairing. The quantum $D$-module ${ }^{1} \mathrm{QDM}^{\mathrm{an}}(\mathfrak{X})$ of $\mathfrak{X}$ is, roughly speaking, the module of sections of this vector bundle equipped with the meromorphic

\footnotetext{
${ }^{1}$ Here we assume the convergence and the analyticity of quantum cohomology (which are true for toric DM stacks); the superscript "an" means analytic.
} 
flat connection $\nabla$ and the pairing $P$ (see Sections 2.3 and 6.1 for the details). We also obtain the formal quantum D-module (or more precisely, the quantum D-module completed in $z$ ) by restricting $\mathrm{QDM}^{\mathrm{an}}(\mathfrak{X})$ to the formal neighbourhood of $z=0$ :

$$
\overline{\mathrm{QDM}^{\mathrm{an}}}(\mathfrak{X})=\mathrm{QDM}^{\mathrm{an}}(\mathfrak{X}) \otimes_{\mathcal{O}}^{\mathrm{an}[z]} \mathcal{O}^{\mathrm{an}} \llbracket z \rrbracket .
$$

When a torus $\mathbb{T}$ acts on $\mathfrak{X}$, we can also define the $\mathbb{T}$-equivariant quantum $\mathrm{D}$-module $\mathrm{QDM}_{\mathbb{T}}^{\mathrm{an}}(\mathfrak{X})$ and its formal version $\overline{\mathrm{QDM}_{\mathbb{T}}^{a n}}(\mathfrak{X})$; they are deformation of the non-equivariant quantum Dmodules.

\subsection{Global Landau-Ginzburg models and toric wall-crossings}

A smooth semiprojective toric Deligne-Mumford (DM) stack $\mathfrak{X}_{\boldsymbol{\Sigma}}$ (in the sense of Borisov-ChenSmith [14]) can be defined as the geometric invariant theory (GIT) quotient of a vector space $\mathbb{C}^{S}$ by a torus $\mathbb{L}_{\mathbb{C}^{\times}}=\mathbb{L} \otimes \mathbb{C}^{\times}$, where $\mathbb{L}$ is a free $\mathbb{Z}$-module of finite rank. The torus $\mathbb{L}_{\mathbb{C}^{\times}}$acts on $\mathbb{C}^{S}$ via a group homomorphism $\mathbb{L}_{\mathbb{C}^{\times}} \rightarrow\left(\mathbb{C}^{\times}\right)^{S}$, and by dualizing it, we get the family pr: $\left(\mathbb{C}^{\times}\right)^{S} \rightarrow$ $\mathbb{L}^{\star} \otimes \mathbb{C}^{\times}$of tori equipped with the function $F=\sum_{b \in S} u_{b}$ on $\left(\mathbb{C}^{\times}\right)^{S}$, where $u_{b}$ is the $b$ th co-ordinate on $\left(\mathbb{C}^{\times}\right)^{S}$

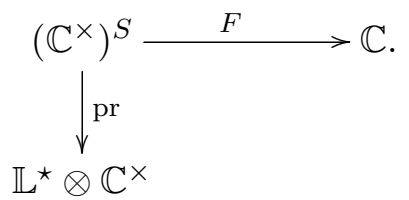

This is the Landau-Ginzburg (LG) model mirror to $\mathfrak{X}_{\boldsymbol{\Sigma}}$ introduced by Givental [47]. Using the secondary fan of Gelfand-Kapranov-Zelevinsky [45], we can partially compactify this LG model to an LG model of the form (see Section 3.2)

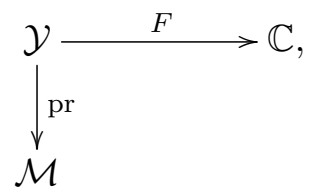

where $\mathcal{Y}, \mathcal{M}$ are possibly singular toric DM stacks (in the sense of Tyomkin [106]). The base $\mathcal{M}$ of this LG model corresponds to the (extended) Kähler moduli space of $\mathfrak{X}_{\boldsymbol{\Sigma}}$ (i.e., the base of the quantum D-module) and contains a distinguished point $0_{\boldsymbol{\Sigma}}$ called the large radius limit point of $\mathfrak{X}_{\boldsymbol{\Sigma}}$. It also contains, ${ }^{2}$ as torus-fixed points, the large radius limit points $0_{\boldsymbol{\Sigma}^{\prime}}$ of several other toric DM stacks $\mathfrak{X}_{\Sigma^{\prime}}$ which can be obtained from $\mathfrak{X}_{\boldsymbol{\Sigma}}$ by varying the stability condition for the GIT quotient $\left[\mathbb{C}^{S} / / \mathbb{L}_{\mathbb{C}^{\times}}\right]$. Hodge-theoretic mirror symmetry for toric DM stacks established by Coates-Corti-Iritani-Tseng $\left[26,27\right.$ implies that, for each smooth toric DM stack $\mathfrak{X}_{\boldsymbol{\Sigma}^{\prime}}$ whose large radius limit point appears in $\mathcal{M}$, we have a mirror map defined on the formal neighbourhood of $0_{\Sigma^{\prime}}$

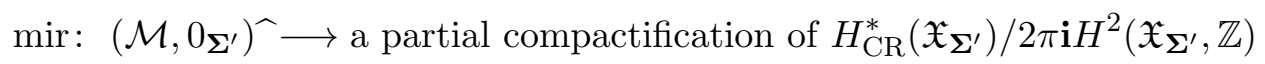

and a mirror isomorphism

$$
\operatorname{Bri}(F){\widehat{\Sigma^{\prime}}} \cong \operatorname{mir}^{*} \operatorname{QDM}^{\text {an }}\left(\mathfrak{X}_{\Sigma^{\prime}}\right) .
$$

\footnotetext{
${ }^{2}$ In fact, by choosing a suitable presentation of $\mathfrak{X}_{\boldsymbol{\Sigma}}$ as the GIT quotient $\left[\mathbb{C}^{S} / / \mathbb{L}_{\mathbb{C}^{\times}}\right]$, we can arrange that the large radius limit point of any given smooth toric DM stack $\mathfrak{X}_{\Sigma^{\prime}}$ having the same affinization and the same generic stabilizer as $\mathfrak{X}_{\boldsymbol{\Sigma}}$ appears in the base space $\mathcal{M}$.
} 
Here $\operatorname{Bri}(F)$ denotes the Brieskorn module associated with the LG potential $F$ and the sub/superscripts $\widehat{\boldsymbol{\Sigma}^{\prime}}$ means the completion at the large-radius limit point $0_{\boldsymbol{\Sigma}^{\prime}}$ (see Sections 4.1-4.2). Using the convergence result from [59, 27], we show that this mirror isomorphism can be extended to a small analytic neighbourhood of $0_{\boldsymbol{\Sigma}^{\prime}}$ as an isomorphism ${ }^{3}$ between a certain analytified Brieskorn module $\overline{\mathrm{Bri}^{\mathrm{an}}}(F)_{\boldsymbol{\Sigma}^{\prime}}$ and the formal quantum $D$-module $\overline{\mathrm{QDM}^{\mathrm{an}}}\left(\mathfrak{X}_{\boldsymbol{\Sigma}^{\prime}}\right.$ ) (see Theorem 4.34). This enables us to compare the quantum D-modules of various birational models of $\mathfrak{X}_{\Sigma}$ over the mirror moduli space $\mathcal{M}$. Then we arrive at the following result (in this theorem, we do not assume compactness of $\mathfrak{X}_{ \pm}$or (semi-)positivity of $c_{1}\left(\mathfrak{X}_{ \pm}\right)$).

Theorem 1.1 (Theorem 5.16). Let $\varphi: \mathfrak{X}_{+} \rightarrow \mathfrak{X}_{-}$be a discrepant transformation between semiprojective toric DM stacks induced by a single wall-crossing in the space of GIT stability conditions. Suppose that $K_{\mathfrak{X}_{+}}>K_{\mathfrak{X}_{-}}$. Then we have a formal decomposition of the $\mathbb{T}$-equivariant quantum D-modules

$$
\operatorname{mir}_{+}^{*} \overline{\mathrm{QDM}_{\mathbb{T}}^{\mathrm{an}}}\left(\mathfrak{X}_{+}\right) \cong \operatorname{mir}_{-}^{*} \overline{\mathrm{QDM}_{\mathbb{T}}^{\mathrm{an}}}\left(\mathfrak{X}_{-}\right) \oplus \mathscr{R}
$$

over a non-empty open subset $\mathcal{U}_{0}^{\prime}$ of $\mathcal{M} \times$ Lie $\mathbb{T}$, where $\operatorname{mir}_{ \pm}$denotes the mirror map for $\mathfrak{X}_{ \pm}$and $\mathscr{R}$ is a locally free $\mathcal{O}_{\mathcal{U}_{0}^{\prime}}^{\mathrm{an}} \llbracket z \rrbracket$-module equipped with a meromorphic flat connection and a pairing.

This theorem is a generalization of the result of González-Woodward [51] who showed a decomposition of the quantum cohomology algebras under a running of the toric minimal model programme. In this theorem, we consider analytic continuation over a neighbourhood of a toric curve $\mathcal{C} \subset \mathcal{M}$ connecting the large radius limit points $0_{ \pm}$for $\mathfrak{X}_{ \pm}$. When $\varphi$ is an isomorphism in codimension one ("flip"), the curve $\mathcal{C}$ is asymptotically close, near the large radius limit points $0_{ \pm}$, to the curve in the boundary of the Kähler moduli space given by the extremal curve class. When $\varphi\left(\right.$ or $\varphi^{-1}$ ) contracts a divisor, the curve $\mathcal{C}$ is asymptotically close to the curve corresponding to the extremal class near $0_{+}$(resp. $0_{-}$) and to the line spanned by a cohomology class of degree greater than 2 near $0_{-}$(resp. of degree less than 2 near $0_{+}$), see Remark 5.9. In either case, at least one of the mirror maps mir $_{ \pm}$involves negative degree variables with respect to the Euler vector field (an instance of the generalized mirror transformation [28,61, 69]) and the formal decomposition occurs over the base of the big quantum cohomology in general. We also note that the decomposition (1.1) is defined only over the formal power series ring $\mathbb{C} \llbracket z \rrbracket$ and the completion in $z$ is unavoidable. In fact, as Theorem 1.3 below shows, the Stokes structure does not admit an orthogonal decomposition.

Using the Givental-Teleman formula [19, 49, 104, 109], we obtain a decomposition for the (all-genus) ancestor Gromov-Witten potentials. The result is stated in terms of Givental's quantization formalism; we refer to Section 5.5 for the notation.

Theorem 1.2 (Theorem 5.19). Let $\mathfrak{X}_{ \pm}$be toric DM stacks as in Theorem 1.1. Let $\mathscr{A}_{ \pm, \tau}$ denote the ancestor potentials of $\mathfrak{X}_{ \pm}$at $\tau \in H_{\mathrm{CR}, \mathbb{T}}^{*}\left(\mathfrak{X}_{ \pm}\right)$. For $(q, \chi) \in \mathcal{M} \times$ Lie $\mathbb{T}$ in a non-empty open set, we have

$$
T_{\mathbf{s}} \widehat{U}_{q, \chi} \mathscr{A}_{+, \operatorname{mir}_{+}(q, \chi)}=\mathscr{A}_{-, \text {mir }_{-}(q, \chi)} \otimes \mathscr{T}^{\otimes \operatorname{rank} \mathscr{R}}
$$

where $\chi$ is the $\mathbb{T}$-equivariant parameter, $\widehat{U}_{q, \chi}$ is the quantization of a symplectic transformation associated with the decomposition (1.1), $\mathscr{T}$ is the Witten-Kontsevich tau-function (the ancestor potential of a point) and $T_{\mathbf{S}}$ is a certain shift operator.

\footnotetext{
${ }^{3}$ The analytified Brieskorn module $\overline{\operatorname{Bri}^{a n}}(F)_{\Sigma^{\prime}}$ is analytic in the $\mathcal{M}$-direction but is still formal in $z$; similarly the formal quantum D-module $\overline{\mathrm{QDM}^{\mathrm{an}}}\left(\mathfrak{X}_{\Sigma^{\prime}}\right)$ is formal in $z$ but is still analytic in $\tau$, so they can be compared.
} 


\subsection{Analytic lift and Orlov's decomposition}

The non-equivariant quantum D-module has (in general) irregular singularities at $z=0$ and the formal quantum D-module misses analytic information such as the Stokes structure at $z=0$. For a compact toric DM stack, at least in the non-equivariant limit and over the semisimple locus, the formal structure of the quantum D-module is very poor ${ }^{4}$, since it is determined only by eigenvalues of the Euler multiplication. We will restore the missing information by describing the analytic lift of the formal decomposition (1.1). By the Hukuhara-Turrittin theorem (see Section 6.1), the decomposition (1.1) in the non-equivariant limit can be locally lifted to an analytic isomorphism: ${ }^{5}$

$$
\left.\left.\operatorname{mir}_{+}^{*} \mathrm{QDM}^{\mathrm{an}}\left(\mathfrak{X}_{+}\right)\right|_{B \times I} \cong \operatorname{mir}_{-}^{*} \mathrm{QDM}^{\mathrm{an}}\left(\mathfrak{X}_{-}\right)\right|_{B \times I} \oplus \mathscr{R}^{\mathrm{an}}
$$

where $B$ is a small open subset of $\mathcal{M}$ and $I$ is an angular sector $\left\{z:|\arg (z)-\phi|<\frac{\pi}{2}+\epsilon\right\}$ with $\epsilon>0$. We call it the analytic lift or a sectorial decomposition; its uniqueness is ensured by the fact that the angle of the sector is bigger than $\pi$. The analytic lift induces a decomposition (depending on $B$ and $I$ ) of the local system underlying $\operatorname{QDM}^{\text {an }}\left(\mathfrak{X}_{+}\right)$. On the other hand, the $\widehat{\Gamma}$-integral structure $[62,70]$ identifies the complexified topological $K$-group $K(\mathfrak{X}) \otimes \mathbb{C}$ with the space of multi-valued flat sections of the quantum D-module; for toric stacks, it corresponds to the integral structure on the GKZ system identified by Borisov-Horja [15]. We show in some special cases that the decomposition of the local system given by the analytic lift corresponds to a semiorthogonal decomposition of the topological $K$-group $K\left(\mathfrak{X}_{+}\right)$via the $\widehat{\Gamma}$-integral structure. An important ingredient here is the fact [62] that the $\widehat{\Gamma}$-integral structure coincides with the natural integral structure of the Brieskorn module under mirror symmetry. By describing the analytic lift in terms of mirror oscillatory integrals and studying the relationship between the local systems of Lefschetz thimbles (see Theorems 7.22 and 7.31), we obtain the following result.

Theorem 1.3 (Theorems 7.25, 7.31, and 7.33). Let $\mathfrak{X}_{-}$be a weak-Fano compact toric stack satisfying a mild technical assumption as described in Section 7.1 and let $\varphi: \mathfrak{X}_{+} \rightarrow \mathfrak{X}_{-}$be a weighted blowup along a toric substack $Z \subset \mathfrak{X}_{-}$. We assume that $\mathfrak{X}_{+}$is also weak-Fano. Then there exist a submersion $f$ from an open set $W$ of $H_{\mathrm{CR}}^{*}\left(\mathfrak{X}_{+}\right)$to $H_{\mathrm{CR}}^{*}\left(\mathfrak{X}_{-}\right)$and an angular sector $I$ (of angle greater than $\pi$ ) such that we have an analytic decomposition over the sector

$$
\left.\left.\mathrm{QDM}^{\mathrm{an}}\left(\mathfrak{X}_{+}\right)\right|_{W \times I} \cong \mathscr{R}_{-J}^{\mathrm{an}} \oplus \cdots \oplus \mathscr{R}_{-1}^{\mathrm{an}} \oplus f^{*} \mathrm{QDM}^{\mathrm{an}}\left(\mathfrak{X}_{-}\right)\right|_{W \times I},
$$

which induces, via the $\widehat{\Gamma}$-integral structure, a semiorthogonal decomposition of the $K$-group

$$
K\left(\mathfrak{X}_{+}\right)=K(Z)_{-J} \oplus \cdots \oplus K(Z)_{-1} \oplus \varphi^{*} K\left(\mathfrak{X}_{-}\right)
$$

where $K(Z)_{k}=\mathcal{O}(-k E) \otimes i_{E_{*}} \varphi_{E}^{*} K(Z) \subset K\left(\mathfrak{X}_{+}\right)$. Here $E$ is the exceptional divisor of $\varphi$, $\varphi_{E}=\left.\varphi\right|_{E}: E \rightarrow Z, i_{E}: E \rightarrow \mathfrak{X}_{+}$is the inclusion and $J=k_{0}+\cdots+k_{c}-1$ when a fibre of $\varphi_{E}: E \rightarrow Z$ is given by the weighted projective space $\mathbb{P}\left(k_{0}, \ldots, k_{c}\right)$.

\section{Remark 1.4.}

(1) In this theorem, we allow $Z$ to be of codimension one (i.e., a toric divisor); in this case $\mathfrak{X}_{+}$ is obtained from $\mathfrak{X}_{-}$by a root construction (see [21]) along $Z$.

\footnotetext{
${ }^{4}$ This does not mean that Theorem 1.1 is trivial. It compares the equivariant quantum D-modules over the open set $\mathcal{U}_{0}^{\prime}$ that contains the non-semisimple loci. It is also important that the two quantum $\mathrm{D}$-modules are connected through the explicit mirror LG model.

${ }^{5}$ More precisely, the analytic lift is defined over functions in $z$ which admit asymptotic expansions along an angular sector (with vertex at $z=0$ ); such functions form a sheaf $\mathcal{A}$ over the real oriented blowup of $\mathbb{C}$ at the origin [95].
} 
(2) When $\mathfrak{X}_{-}$is a smooth projective variety and $\varphi: \mathfrak{X}_{+} \rightarrow \mathfrak{X}_{-}$is the blowup along a smooth subvariety $Z$, the decomposition (1.3) is induced by Orlov's semiorthogonal decomposition [87] for $D^{b}\left(\mathfrak{X}_{+}\right)$. In general, the Orlov-type decomposition (1.3) arises from a sectorial decomposition of the quantum D-module at a point which is far from the large radius limit point. On the other hand, we can idenfity explicitly the locus in the mirror moduli space $\mathcal{M}$ where the analytic lift $(1.2)$ induces the pull-back $\varphi^{*}: K\left(\mathfrak{X}_{-}\right) \rightarrow K\left(\mathfrak{X}_{+}\right)$in $K$-theory (Theorem 7.25).

(3) The result suggests that each residual piece $\mathscr{R}_{i}^{\text {an }}$ of the sectorial decomposition should be related to the quantum D-module of the blowup centre $Z$; they certainly have the same formal structure, but we do not know if the Stokes structures are related (although we expect from homological mirror symmetry that they should be related).

(4) We need the weak-Fano assumption when we apply results from [62]. We hope that the same result holds without such assumptions, but it may require some technical advances.

(5) In Section 8, we formulate a general conjecture relating the decomposition of topological $K$-groups and that of quantum D-modules under discrepant transformations. Under the conjecture, the decomposition of $K$-groups in principle determines the relationship between the quantum D-modules of $\mathfrak{X}_{+}$and $\mathfrak{X}_{-}$(including the map $f$ ). This involves solving a Riemann-Hilbert problem; see Proposition 8.5.

\subsection{Related works}

We mention some of the earlier works that are closely related to the present paper.

The relationship between quantum cohomology and derived category has been suggested by Dubrovin [39]. Our Theorem 1.3 can be viewed as a variation on this theme (see also Gamma conjecture [44] or Dubrovin-type conjecture [101]). Bayer [12] showed that the semisimplicity of quantum cohomology is preserved under blowup at a point in connection with Dubrovin's conjecture [39]. His computation [12, Lemma 3.4.2] for the spectral cover is compatible with the picture in this paper.

As mentioned earlier, González-Woodward [51] showed a decomposition of toric quantum cohomology under flips; they used the same LG model mirrors (combined with the quantum Kirwan map) to analyze the change of quantum cohomology. Charest-Woodward [22] and Sanda [99, 100] discussed (orthogonal) decomposition of Fukaya category and of quantum cohomology under flips/blowups. From a categorical viewpoint, the quantum cohomology (or the formal quantum D-module) should arise as the Hochschild (resp. negative cyclic) homology of the Fukaya category, and a decomposition of the Fukaya category should induce a (formal) decomposition of the quantum cohomology/D-module via the open-closed map.

Acosta-Shoemaker [4, 5] (see also an earlier work of Acosta [3]) studied discrepant wallcrossings for toric Gromov-Witten theory in the same setting as ours. They compared the Givental $I$-functions of $\mathfrak{X}_{ \pm}$over the mirror moduli space $\mathcal{M}$. Writing $I_{ \pm}$for the $I$-functions of $\mathfrak{X}_{ \pm}$, they showed that the asymptotic expansion of $I_{+}$near the large-radius limit point $0_{-}$is related to $I_{-}$by a linear transformation $L: H_{\mathrm{CR}}^{*}\left(\mathfrak{X}_{+}\right) \rightarrow H_{\mathrm{CR}}^{*}\left(\mathfrak{X}_{-}\right)$, i.e., $L I_{+}(q) \sim I_{-}(q)$ as $q \rightarrow 0_{-}$along some (one-dimensional) angular direction. We expect that their linear transformation $L$ should correspond to the projection between the quantum cohomology local systems for $\mathfrak{X}_{ \pm}$associated with an analytic lift (1.2). They also dealt with the case of complete intersections in toric stacks (which we do not cover in this paper). More recently, Lee-Lin-Wang [78, Section 6], [79] have announced a decomposition of quantum D-modules under flips and showed it for local toric flips. In these approaches $[3,4,5,78,79]$, they studied irregular singularities on the base $\mathcal{M}$ directly whereas we studied those on the $z$-plane; the singularities at $z=0$ and $q=0_{-}$are closely related, but those at $z=0$ have a simpler structure. 
Recently, Clingempeel-Le Floch-Romo [25] compared the hemisphere partition functions (which in our language correspond to certain solutions of the quantum D-modules) of the cyclic quotient singularities $\mathbb{C}^{2} / \mu_{n}$ and their Hirzebruch-Jung resolutions. They discussed the relation to a semiorthogonal decomposition of the derived categories, extending the work of Herbst-HoriPage [56] to the anomalous (discrepant) case. Their examples are complementary to ours: the Hirzebruch-Jung resolutions are type (II-ii) discrepant transformations whereas transformations in Theorem 1.3 are of type (II-i) or (III) (see Remark 5.4 for these types).

Under homological mirror symmetry, the derived categories of coherent sheaves for toric stacks correspond to the Fukaya-Seidel categories of the mirror LG models. Kerr [73], DiemerKatzarkov-Kerr [36, 35] and Ballard-Diemer-Favero-Katzarkov-Kerr [8] (see also [9]) studied semiorthogonal decompositions of the Fukaya-Seidel categories of the LG mirrors under toric wall-crossings; they conjectured [8, Conjecture 1] that semiorthogonal decompositions for the Fukaya-Seidel categories and for the derived categories should match up under mirror symmetry. Theorem 1.3 can be viewed as an evidence for their conjecture on the level of enumerative mirror symmetry. We note that they introduced a similar toric compactification of the moduli space of LG models and studied how the critical values assemble under deformation in a more general setting than ours (a relevant discussion appears in Section 7.4 in this paper).

After the author finished a draft of this paper, he heard a talk of Kontsevich [74] who studied (in joint project with Katzarkov and Pantev) the change of quantum cohomology under blowups from a similar perspective and gave an application to birational geometry.

The author apologizes for the long delay in preparing the paper since the original announcement [60] (see also [63]) in June 2008. There were many technical issues in proving our results in this generality. Advances made in joint work [26, 27] with Coates, Corti and Tseng and in joint work [44] with Galkin and Golyshev are essential in this paper.

\section{Preliminaries}

In this section, in order to fix notation, we review quantum cohomology, quantum D-modules and the Gamma-integral structure. Our main interest in this paper lies in the case where $\mathfrak{X}$ is a toric DM stack, but all the materials in this section make sense for a general smooth DM stack $\mathfrak{X}$ satisfying mild assumptions.

\subsection{Quantum cohomology}

Gromov-Witten theory for smooth Deligne-Mumford stacks (or symplectic orbifolds) has been developed by Chen-Ruan [23] and Abramovich-Graber-Vistoli [1,2]. We use the algebrogeometric approach in $[1,2]$.

Let $\mathfrak{X}$ be a smooth DM (Deligne-Mumford) stack over $\mathbb{C}$. We write $X$ for the coarse moduli space of $\mathfrak{X}$. Recall that the inertia stack $I \mathfrak{X}$ is the fibre product $\mathfrak{X} \times \mathfrak{X} \times \mathfrak{X} \mathfrak{X}$ of the two diagonal morphisms $\mathfrak{X} \rightarrow \mathfrak{X} \times \mathfrak{X}$. A point on $I \mathfrak{X}$ is given by a pair $(x, g)$ of a point $x \in \mathfrak{X}$ and a stabilizer $g \in \operatorname{Aut}(x)$. We write $I \mathfrak{X}=\bigsqcup_{v \in \text { Box }} \mathfrak{X}_{v}$ for the decomposition of $I \mathfrak{X}$ into connected components, where Box is the index set. We have a distinguished element $0 \in$ Box that corresponds to the untwisted sector $\mathfrak{X}_{0} \cong \mathfrak{X}$ consisting of points $(x, g=1)$ with the trivial stabilizer. The orbifold cohomology $H_{\mathrm{CR}}^{*}(\mathfrak{X})$ of Chen and Ruan [24] is defined to be

$$
H_{\mathrm{CR}}^{*}(\mathfrak{X}):=H^{*-2 \operatorname{age}}(I \mathfrak{X}, \mathbb{C})=\bigoplus_{v \in \operatorname{Box}} H^{*-2 \operatorname{age}(v)}\left(\mathfrak{X}_{v}, \mathbb{C}\right),
$$

where age: $I \mathfrak{X} \rightarrow \mathbb{Q}_{\geq 0}$ is a locally constant function giving a shift of degrees and we write $\operatorname{age}(v)=\left.\operatorname{age}\right|_{\mathfrak{x}_{v}}$ (see Section 3.1.3 for the age in the case of toric DM stacks). The right-hand 
side means the cohomology group of the underlying complex analytic space of $I \mathfrak{X}$ and we use complex coefficients unless otherwise specified. We also restrict ourselves to cohomology classes of "even parity", i.e., we only consider cohomology classes of even degrees ${ }^{6}$ on $I \mathfrak{X}$. For toric DM stacks, every orbifold cohomology class has even parity. When $\mathfrak{X}$ is proper, the orbifold Poincaré pairing on $H_{\mathrm{CR}}^{*}(\mathfrak{X})$ is defined to be

$$
(\alpha, \beta):=\int_{I \mathfrak{X}} \alpha \cup \operatorname{inv}^{*} \beta,
$$

where inv: $I \mathfrak{X} \rightarrow I \mathfrak{X}$ is the involution sending $(x, g)$ to $\left(x, g^{-1}\right)$. For $d \in H_{2}(\mathfrak{X}, \mathbb{Q})$ and $l \in \mathbb{Z}_{>0}$, let $\mathfrak{X}_{g, l, d}$ denote the moduli stack of genus- $g$ twisted stable maps to $\mathfrak{X}$ of degree $d$ and with $l$ marked points (this was denoted by $\mathcal{K}_{g, l}(\mathfrak{X}, d)$ in [2]). It carries a virtual fundamental class $\left[\mathfrak{X}_{g, l, d}\right]_{\text {vir }} \in A_{*}\left(\mathfrak{X}_{g, l, d}, \mathbb{Q}\right)$ and the evaluation maps $\mathrm{ev}_{i}: \mathfrak{X}_{g, l, d} \rightarrow \bar{I} \mathfrak{X}, i=1, \ldots, l$ to the rigidified cyclotomic inertia stack $\bar{I} \mathfrak{X}$ (see [2, Section 3.4]). When the moduli stack $\mathfrak{X}_{g, l, d}$ is proper (this happens when $\mathfrak{X}$ has a projective coarse moduli space), we define genus-zero descendant GromovWitten invariants by

$$
\left\langle\alpha_{1} \psi^{k_{1}}, \ldots, \alpha_{l} \psi^{k_{l}}\right\rangle_{g, l, d}:=\int_{\left[\mathfrak{X}_{g, l, d}\right]_{\mathrm{vir}}} \prod_{i=1}^{l} \mathrm{ev}_{i}^{*}\left(\alpha_{i}\right) \psi_{i}^{k_{i}},
$$

where $\alpha_{1}, \ldots, \alpha_{l} \in H_{\mathrm{CR}}^{*}(\mathfrak{X}), d \in H_{2}(\mathfrak{X}, \mathbb{Q})$ and $\psi_{i}$ denotes the $\psi$-class (see [2, Section 8.3]) at the $i$ th marking. Here note that the rigidified cyclotomic inertia stack $\bar{I} \mathfrak{X}$ has the same coarse moduli space as $I \mathfrak{X}$, and thus $\alpha_{i}$ can be regarded as a cohomology class of $\bar{I} \mathfrak{X}$.

We assume that there exists a finitely generated monoid $\boldsymbol{\Lambda}_{+} \subset H_{2}(\mathfrak{X}, \mathbb{Q})$ such that $\mathbb{R}_{\geq 0} \boldsymbol{\Lambda}_{+}$is a strictly convex full-dimensional cone in $H_{2}(\mathfrak{X}, \mathbb{R})$ and that $\boldsymbol{\Lambda}_{+}$contains classes of any orbifold stable curves. We also assume that $\boldsymbol{\Lambda}_{+}$is saturated, i.e., $\boldsymbol{\Lambda}_{+}=\boldsymbol{\Lambda} \cap \mathbb{R}_{\geq 0} \boldsymbol{\Lambda}_{+}$for $\boldsymbol{\Lambda}:=\mathbb{Z} \boldsymbol{\Lambda}_{+}$. The monoid $\boldsymbol{\Lambda}_{+}$for toric DM stacks will be described in Section 3.3 and will be denoted by $\boldsymbol{\Lambda}_{+}^{\boldsymbol{\Sigma}}$ there. For a ring $K$, let $K \llbracket \boldsymbol{\Lambda}_{+} \rrbracket$ denote the completion of $K\left[\boldsymbol{\Lambda}_{+}\right]$consisting of all formal sums $\sum_{d \in \boldsymbol{\Lambda}_{+}} a_{d} Q^{d}$ with $a_{d} \in K$. The variable $Q$ here is called the Novikov variable. We also choose a homogeneous basis $\left\{\phi_{i}\right\}_{i=0}^{s}$ of $H_{\mathrm{CR}}^{*}(\mathfrak{X})$ and introduce linear co-ordinates $\left\{\tau^{i}\right\}_{i=0}^{s}$ on $H_{\mathrm{CR}}^{*}(\mathfrak{X})$ as $\left(\tau^{0}, \ldots, \tau^{s}\right) \mapsto \tau=\sum_{i=0}^{s} \tau^{i} \phi_{i}$. We write $K \llbracket \tau \rrbracket=K \llbracket \tau^{0}, \ldots, \tau^{s} \rrbracket$ for any ring $K$. The quantum product $\alpha \star \beta$ of classes $\alpha, \beta \in H_{\mathrm{CR}}^{*}(\mathfrak{X})$ is defined so that for every $\gamma \in H_{\mathrm{CR}}^{*}(\mathfrak{X})$, we have

$$
(\alpha \star \beta, \gamma):=\sum_{d \in \Lambda_{+}} \sum_{l \geq 0}\langle\alpha, \beta, \gamma, \tau, \ldots, \tau\rangle_{0, l+3, d} \frac{Q^{d}}{l !},
$$

where $\tau=\sum_{i=0}^{s} \tau^{i} \phi_{i} \in H_{\mathrm{CR}}^{*}(\mathfrak{X})$ is a parameter. This definition makes sense when $X$ is projective and $\alpha \star \beta$ lies in $H_{\mathrm{CR}}^{*}(\mathfrak{X}) \otimes \mathbb{C} \llbracket \boldsymbol{\Lambda}_{+} \rrbracket \llbracket \tau \rrbracket$. The quantum product $\star$ is known to be commutative and associative and defines a commutative ring structure on $H_{\mathrm{CR}}^{*}(\mathfrak{X}) \otimes \mathbb{C} \llbracket \boldsymbol{\Lambda}_{+} \rrbracket \llbracket \tau \rrbracket$. When we want to emphasize the dependence of $\star$ on the parameter $\tau$, we shall write $\alpha \star_{\tau} \beta$ in place of $\alpha \star \beta$.

The Gromov-Witten invariants and the quantum product can be generalized to the equivariant setting or to a non-projective space $\mathfrak{X}$. Suppose that an algebraic torus $\mathbb{T}$ acts on $\mathfrak{X}$. The equivariant orbifold cohomology $H_{\mathrm{CR}, \mathbb{T}}^{*}(\mathfrak{X})$ is defined to be the $\mathbb{T}$-equivariant cohomology of $I \mathfrak{X}$ with the same degree shift as before:

$$
H_{\mathrm{CR}, \mathbb{T}}^{*}(\mathfrak{X}):=H_{\mathbb{T}}^{*-2 \text { age }}(I \mathfrak{X}, \mathbb{C})=\bigoplus_{v \in \operatorname{Box}} H_{\mathbb{T}}^{*-2 \text { age }(v)}\left(\mathfrak{X}_{v}, \mathbb{C}\right) .
$$

Let $E \mathbb{T} \rightarrow B \mathbb{T}$ denote a universal $\mathbb{T}$-bundle. We assume that

\footnotetext{
${ }^{6}$ An element $\alpha \in H_{\mathrm{CR}}^{*}(\mathfrak{X})$ having "even parity" does not imply that the degree of $\alpha$ as an orbifold cohomology class is even; it means that the degree of $\alpha$ is even as an element of $H^{*}(I \mathfrak{X})$.
} 
(a) $I \mathfrak{X}$ is equivariantly formal, i.e., the Serre spectral sequence for $I \mathfrak{X} \times \mathbb{T} E \mathbb{T} \rightarrow B \mathbb{T}$ collapses at the $E^{2}$-term $($ over $\mathbb{Q})$; this implies that $H_{\mathrm{CR}, \mathbb{T}}^{*}(\mathfrak{X})$ is a free $R_{\mathbb{T}}:=H_{\mathbb{T}}^{*}(\mathrm{pt}, \mathbb{C})$-module of rank $\operatorname{dim} H_{\mathrm{CR}}^{*}(\mathfrak{X})$;

(b) the $\mathbb{T}$-fixed set of $\mathfrak{X}$ is projective;

(c) the evaluation maps $\mathrm{ev}_{i}: \mathfrak{X}_{0, l, d} \rightarrow \bar{I} \mathfrak{X}$ are proper.

By the assumption (a), the $\mathbb{C}$-basis $\left\{\phi_{i}\right\}_{i=0}^{s} \subset H_{\mathrm{CR}}^{*}(\mathfrak{X})$ can be lifted to an $R_{\mathbb{T}^{-}}$-basis of $H_{\mathrm{CR}, \mathbb{T}}^{*}(\mathfrak{X})$, which we denote by the same symbol. This induces $R_{\mathbb{T}}$-linear co-ordinates $\left(\tau^{0}, \ldots, \tau^{s}\right) \mapsto \tau=$ $\sum_{i=0}^{s} \tau^{i} \phi_{i}$ on $H_{\mathrm{CR}, \mathbb{T}}^{*}(\mathfrak{X})$ as before. Under the assumption (b), we can define the equivariant orbifold Poincaré pairing and the equivariant Gromov-Witten invariants via the virtual localization formula [53]. They take values in the fraction field $S_{\mathbb{T}}:=\operatorname{Frac}\left(R_{\mathbb{T}}\right)$ of $R_{\mathbb{T}}$. Under the assumption (c), we can define the quantum product using the push-forward by the proper map ev 3 as follows:

$$
\alpha \star \beta=\sum_{d \in \boldsymbol{\Lambda}_{+}} \sum_{l \geq 0} \operatorname{inv}^{*} \operatorname{PD~ev}_{3 *}\left(\operatorname{ev}_{1}^{*}(\alpha) \operatorname{ev}_{2}^{*}(\beta) \prod_{j=1}^{l} \operatorname{ev}_{3+j}^{*}(\tau) \cap\left[\mathfrak{X}_{0, l+3, d}\right]_{\operatorname{vir}}\right) \frac{Q^{d}}{l !},
$$

where PD stands for the Poincaré duality on $I \mathfrak{X}$. Therefore we get the quantum product $\star$ defined on the space $H_{\mathrm{CR}, \mathbb{T}}^{*}(\mathfrak{X}) \otimes_{R_{\mathbb{T}}} R_{\mathbb{T}} \llbracket \boldsymbol{\Lambda}_{+} \rrbracket \llbracket \tau \rrbracket$ without inverting equivariant parameters, whereas the Gromov-Witten invariants themselves lie in $S_{\mathbb{T}}$ in general.

Remark 2.1. As remarked in [20], the assumption (c) is satisfied if the coarse moduli space $X$ is semi-projective, i.e., projective over an affine variety. We will impose the semi-projectivity assumption on toric DM stacks.

\subsection{Kähler moduli space}

In this section, we specialize the Novikov variable $Q$ to one with the aid of the divisor equation, and introduce the Kähler moduli space $\mathcal{M}_{\mathrm{A}}(\mathfrak{X})$ parameterizing the quantum product.

As before, let $\phi_{0}, \phi_{1}, \ldots, \phi_{s}$ be a homogeneous basis of $H_{\mathrm{CR}}^{*}(\mathfrak{X})$. We assume that $\phi_{0}=\mathbf{1}$ is the identity class and that $\left\{\phi_{1}, \ldots, \phi_{r}\right\}, r \leq s$ is a basis of the degree-two untwisted sector $H^{2}(\mathfrak{X}) \subset H_{\mathrm{CR}}^{2}(\mathfrak{X})$. We write $\tau=\sigma+\tau^{\prime}$ with $\sigma=\sum_{i=1}^{r} \tau^{i} \phi_{i} \in H^{2}(\mathfrak{X})$ and $\tau^{\prime}=\tau^{0} \phi_{0}+\sum_{i=r+1}^{s} \tau^{i} \phi_{i}$. The divisor equation [2, Theorem 8.3.1] implies

$$
\left(\alpha \star_{\tau} \beta, \gamma\right)=\sum_{d \in \Lambda_{+}} \sum_{l=0}^{\infty}\left\langle\alpha, \beta, \gamma, \tau^{\prime}, \ldots, \tau^{\prime}\right\rangle_{0, l+3, d} \frac{Q^{d} e^{\sigma \cdot d}}{l !}
$$

This shows that the quantum product $\star_{\tau}$ depends only on the equivalence class

$$
[\tau] \in H_{\mathrm{CR}}^{*}(\mathfrak{X}) / 2 \pi \mathbf{i} \Lambda^{\star},
$$

where $\boldsymbol{\Lambda}^{\star} \subset H^{2}(\mathfrak{X}, \mathbb{Q})$ denotes the dual lattice of $\boldsymbol{\Lambda}=\mathbb{Z} \boldsymbol{\Lambda}_{+}$. Note that $d \in \boldsymbol{\Lambda}$ defines a function $q^{d}:[\tau] \mapsto e^{\sigma \cdot d}=\exp \left(\sum_{i=1}^{r} \tau^{i}\left(\phi_{i} \cdot d\right)\right)$ on $H_{\mathrm{CR}}^{*}(\mathfrak{X}) / 2 \pi \mathbf{i} \boldsymbol{\Lambda}^{\star}$, and that each co-ordinate $\tau^{i}$ with $i \notin$ $\{1, \ldots, r\}$ also defines a function on $H_{\mathrm{CR}}^{*}(\mathfrak{X}) / 2 \pi \mathbf{i} \boldsymbol{\Lambda}^{\star}$. These functions define an open embedding of $H_{\mathrm{CR}}^{*}(\mathfrak{X}) / 2 \pi \mathbf{i} \boldsymbol{\Lambda}^{\star}$ into the following space:

$$
\mathcal{M}_{\mathrm{A}}(\mathfrak{X}):=\operatorname{Spec} \mathbb{C}\left[\boldsymbol{\Lambda}_{+}\right]\left[\tau^{\prime}\right]=\operatorname{Spec} \mathbb{C}\left[\boldsymbol{\Lambda}_{+}\right]\left[\tau^{0}, \tau^{r+1}, \ldots, \tau^{s}\right],
$$

where the element of $\mathbb{C}\left[\boldsymbol{\Lambda}_{+}\right]$corresponding to $d \in \boldsymbol{\Lambda}_{+}$represents the function $q^{d}=e^{\sigma \cdot d}$. The space $\mathcal{M}_{\mathrm{A}}(\mathfrak{X})$ gives a partial compactification of $H_{\mathrm{CR}}^{*}(\mathfrak{X}) / 2 \pi \mathbf{i} \boldsymbol{\Lambda}^{\star}$ depending on the choice of 
the monoid $\boldsymbol{\Lambda}_{+}$. By setting $Q=1$, we may view $\star_{\tau}$ as a family of products parameterized by the formal neighbourhood of the "origin" (that is, $q^{d}=0$ for all non-zero $d \in \boldsymbol{\Lambda}_{+}$and $\left.\tau^{0}=\tau^{r+1}=\cdots=\tau^{s}=0\right)$ in $\mathcal{M}_{\mathrm{A}}(\mathfrak{X})$. This origin is called the large radius limit point.

Quantum cohomology for smooth DM stacks has the additional symmetry called the $\mathrm{Ga}$ lois symmetry [62, Section 2.2]. Let $H^{2}(\mathfrak{X}, \mathbb{Z})$ denote the sheaf cohomology of the constant sheaf $\mathbb{Z}$ on the topological stack (orbifold) underlying $\mathfrak{X}$; an element $\xi \in H^{2}(\mathfrak{X}, \mathbb{Z})$ corresponds to a topological orbi-line bundle $L_{\xi}$ over $\mathfrak{X}$. For a connected component $\mathfrak{X}_{v}$ of $I \mathfrak{X}$, the stabilizer along $\mathfrak{X}_{v}$ acts on fibres of $L_{\xi}$ by a constant scalar $\exp \left(2 \pi \mathbf{i} f_{v}(\xi)\right)$ for some $f_{v}(\xi) \in[0,1) \cap \mathbb{Q}$. This number $f_{v}(\xi)$ is called the age of $L_{\xi}$ along $\mathfrak{X}_{v}$. We define a map $g(\xi): H_{\mathrm{CR}}^{*}(\mathfrak{X}) \rightarrow H_{\mathrm{CR}}^{*}(\mathfrak{X})$ by

$$
g(\xi)(\tau)=\left(\tau_{0}-2 \pi \mathbf{i} \xi\right) \oplus \bigoplus_{v \neq 0} e^{2 \pi \mathbf{i} f_{v}(\xi)} \tau_{v},
$$

where $\tau_{v}$ denotes the $H^{*}\left(\mathfrak{X}_{v}\right)$-component of $\tau \in H_{\mathrm{CR}}^{*}(\mathfrak{X})$ in the decomposition (2.1). Let $d g(\xi) \in$ $\operatorname{End}\left(H_{\mathrm{CR}}^{*}(\mathfrak{X})\right)$ denote the derivative of the map $g(\xi)$; it is given by $d g(\xi) \tau=\bigoplus_{v \in \operatorname{Box}} e^{2 \pi \mathbf{i} f_{v}(\xi)} \tau_{v}$. Gromov-Witten invariants satisfy (see [62, Proposition 2.3])

$$
\left\langle d g(\xi)\left(\alpha_{1}\right) \psi^{k_{1}}, \ldots, d g(\xi)\left(\alpha_{l}\right) \psi^{k_{l}}\right\rangle_{g, l, d}=e^{2 \pi \mathbf{i} \xi \cdot d}\left\langle\alpha_{1} \psi^{k_{1}}, \ldots, \alpha_{l} \psi^{k_{l}}\right\rangle_{g, l, d}
$$

and thus the quantum product satisfies

$$
d g(\xi)\left(\alpha \star_{\tau} \beta\right)=(d g(\xi) \alpha) \star_{g(\xi)(\tau)}(d g(\xi) \beta) .
$$

The map $g(\xi)$ induces the action of $H^{2}(\mathfrak{X}, \mathbb{Z}) / \boldsymbol{\Lambda}^{\star}$ on $H_{\mathrm{CR}}^{*}(\mathfrak{X}) / 2 \pi \mathbf{i} \boldsymbol{\Lambda}^{\star}$; this action naturally extends to the partial compactification $\mathcal{M}_{\mathrm{A}}(\mathfrak{X})$. In view of this symmetry, we can regard the quantum products $\star_{\tau}$ as a family of products parametrized by the formal neighbourhood of the origin (large radius limit) of the stack:

$$
\left[\mathcal{M}_{\mathrm{A}}(\mathfrak{X}) /\left(H^{2}(\mathfrak{X}, \mathbb{Z}) / \boldsymbol{\Lambda}^{\star}\right)\right] \text {. }
$$

We refer to $\mathcal{M}_{\mathrm{A}}(\mathfrak{X})$ or to the quotient stack above as the Kähler moduli space, where the subscript A stands for the A-model.

The above construction can be adapted to the equivariant quantum cohomology. We choose a homogeneous $R_{\mathbb{T}}$-basis $\left\{\phi_{i}\right\}_{i=0}^{s}$ of $H_{\mathrm{CR}, \mathbb{T}}^{*}(\mathfrak{X})$ such that

$$
\phi_{0}=\mathbf{1} \quad \text { and } \quad\left\{\phi_{1}, \ldots, \phi_{r}\right\} \subset H_{\mathbb{T}}^{2}(\mathfrak{X}) \quad \text { with } r=\operatorname{dim} H^{2}(\mathfrak{X}) \leq s .
$$

Then the non-equivariant limits of $\phi_{1}, \ldots, \phi_{r}$ form a basis of $H^{2}(\mathfrak{X})$. The basis $\left\{\phi_{i}\right\}_{i=0}^{s}$ defines $R_{\mathbb{T}^{-}}$-linear co-ordinates $\left\{\tau^{i}\right\}_{i=0}^{s}$ on $H_{\mathrm{CR}, \mathbb{T}}^{*}(\mathfrak{X})$ as before. The equivariant Kähler moduli spaces are given by replacing the ground ring $\mathbb{C}$ with $R_{\mathbb{T}}$.

$$
\mathcal{M}_{\mathrm{A}, \mathbb{T}}(\mathfrak{X}):=\operatorname{Spec} R_{\mathbb{T}}\left[\boldsymbol{\Lambda}_{+}\right]\left[\tau^{0}, \tau^{r+1}, \ldots, t^{s}\right] .
$$

It is fibred over Spec $R_{\mathbb{T}} \cong$ Lie $\mathbb{T}$. The group $H^{2}(\mathfrak{X}, \mathbb{Z}) / \boldsymbol{\Lambda}^{\star}$ acts on $\mathcal{M}_{\mathrm{A}, \mathbb{T}}(\mathfrak{X})$ through the isomorphism $\mathcal{M}_{\mathrm{A}, \mathbb{T}}(\mathfrak{X}) \cong \mathcal{M}_{\mathrm{A}}(\mathfrak{X}) \times \operatorname{Spec} R_{\mathbb{T}}$. The $\mathbb{T}$-equivariant quantum product $\star_{\tau}$ can be viewed as a family of product structures parameterized by the formal neighbourhood of the origin in $\left[\mathcal{M}_{\mathrm{A}, \mathbb{T}}(\mathfrak{X}) /\left(H^{2}(\mathfrak{X}, \mathbb{Z}) / \boldsymbol{\Lambda}^{\star}\right)\right]$.

Remark 2.2. Unlike the non-equivariant case, the equivariant Kähler moduli space $\mathcal{M}_{\mathrm{A}, \mathbb{T}}(\mathfrak{X})$ is not a partial compactification of $H_{\mathrm{CR}, \mathbb{T}}^{*}(\mathfrak{X}) / 2 \pi \mathbf{i} \Lambda^{\star}$; we do not even have a natural map $H_{\mathrm{CR}, \mathbb{T}}^{*}(\mathfrak{X}) \rightarrow \mathcal{M}_{\mathrm{A}, \mathbb{T}}(\mathfrak{X})$. If we regard $H_{\mathrm{CR}, \mathbb{T}}^{*}(\mathfrak{X})$ as a locally free coherent sheaf over Spec $R_{\mathbb{T}}$ and write $\mathbf{H}_{\mathrm{CR}, \mathbb{T}}(\mathfrak{X})=\operatorname{Spec}\left(\operatorname{Sym}_{R_{\mathbb{T}}}^{\bullet}\left(H_{\mathrm{CR}, \mathbb{T}}^{*}(\mathfrak{X})^{\vee}\right)\right)$ for the total space of the associated vector bundle (where $(-)^{\vee}$ stands for the dual as an $R_{\mathbb{T}}$-module), then we have an open dense embedding $\mathbf{H}_{\mathrm{CR}, \mathbb{T}}(\mathfrak{X}) / 2 \pi \mathbf{i} \Lambda^{\star} \rightarrow \mathcal{M}_{\mathrm{A}, \mathbb{T}}(\mathfrak{X})$. In this paper, we take the view that the equivariant quantum product $\star_{\tau}$ is parametrized by points in $\mathbf{H}_{\mathrm{CR}, \mathbb{T}}(\mathfrak{X})$ rather than by equivariant cohomology classes. Note that equivariant cohomology classes correspond to sections of $\mathbf{H}_{\mathrm{CR}, \mathbb{T}}(\mathfrak{X}) \rightarrow \operatorname{Spec} R_{\mathbb{T}}$. 
Remark 2.3. The equivariant Kähler moduli space $\mathcal{M}_{\mathrm{A}, \mathbb{T}}(\mathfrak{X})$ depends on the choice of a basis $\phi_{1}, \ldots, \phi_{r}$. In fact, in the equivariant case, we can replace the basis $\left\{\phi_{i}\right\}_{i=0}^{s}$ with a new basis $\left\{\tilde{\phi}_{i}\right\}_{i=0}^{s}$ of the form

$$
\tilde{\phi}_{i}= \begin{cases}\phi_{i}+c_{i} \phi_{0}, & i \in\{1, \ldots, r\}, \\ \phi_{i}, & i \notin\{1, \ldots, r\},\end{cases}
$$

for some $c_{i} \in H_{\mathbb{T}}^{2}(\mathrm{pt})$ without violating the homogeneity. Then the corresponding 0-th coordinate $\tilde{\tau}^{0}=\tau^{0}-\sum_{i=1}^{r} c_{i} \tau^{i}$ does not lie in the ring $R_{\mathbb{T}}\left[\boldsymbol{\Lambda}_{+}\right]\left[\tau^{0}, \tau^{r+1}, \ldots, \tau^{s}\right]$. In other words, the construction of the equivariant Kähler moduli space requires the choice of a splitting of the sequence $0 \rightarrow H_{\mathbb{T}}^{2}(\mathrm{pt}) \rightarrow H_{\mathbb{T}}^{2}(\mathfrak{X}) \rightarrow H^{2}(\mathfrak{X}) \rightarrow 0$.

Remark 2.4. In the above discussion, we considered the specialization $Q=1$ and the equivalence class of $\tau$ in $H_{\mathrm{CR}}^{*}(\mathfrak{X}) / 2 \pi \mathbf{i} \Lambda^{\star}$. This is equivalent to considering the restriction to $\tau^{1}=\cdots=$ $\tau^{r}=0$ and the substitution of $q^{d}$ for $Q^{d}$.

Remark 2.5. Henceforth we specialize the Novikov variable $Q$ to one in the quantum product $\star_{\tau}$, unless otherwise stated.

\subsection{Quantum D-module}

The quantum product defines a meromorphic flat connection on a vector bundle (with fibre orbifold cohomology) over the Kähler moduli space, called the quantum connection. The quantum connection, the grading and the orbifold Poincaré pairing constitute the quantum D-module of $\mathfrak{X}$.

We start by explaining the equivariant version; we get the non-equivariant version by taking non-equivariant limit. As before, we fix a homogeneous $R_{\mathbb{T}}$-free basis $\left\{\phi_{0}, \ldots, \phi_{s}\right\}$ of $H_{\mathrm{CR}, \mathbb{T}}^{*}(\mathfrak{X})$ that satisfies (2.4). We use this basis to construct the equivariant Kähler moduli spaces $\mathcal{M}_{\mathrm{A}, \mathbb{T}}(\mathfrak{X})$. Consider the vector bundle

$$
\mathbf{H}_{\mathrm{CR}, \mathbb{T}}(\mathfrak{X}) \times_{\mathrm{Spec} R_{\mathbb{T}}}\left(\mathcal{M}_{\mathrm{A}, \mathbb{T}}(\mathfrak{X}) \times \mathbb{C}_{z}\right) \rightarrow \mathcal{M}_{\mathrm{A}, \mathbb{T}}(\mathfrak{X}) \times \mathbb{C}_{z}
$$

of rank equal to $\operatorname{dim} H_{\mathrm{CR}}^{*}(\mathfrak{X})$, where $\mathbb{C}_{z}:=\operatorname{Spec} \mathbb{C}[z]$ is the complex plane with co-ordinate $z$ and $\mathbf{H}_{\mathrm{CR}, \mathbb{T}}(\mathfrak{X})$ denotes a vector bundle over $\operatorname{Spec} R_{\mathbb{T}}$ corresponding to $H_{\mathrm{CR}, \mathbb{T}}^{*}(\mathfrak{X})$ (see Remark 2.2). The Galois symmetry in the previous section induces the $\left(H^{2}(\mathfrak{X}, \mathbb{Z}) / \boldsymbol{\Lambda}^{\star}\right)$-action on this vector bundle defined by the map $d g(\xi) \times g(\xi) \times \operatorname{id}_{\mathbb{C}_{z}}$. By the Galois symmetry, this vector bundle descends to a vector bundle on the quotient stack $\left[\mathcal{M}_{\mathrm{A}, \mathbb{T}}(\mathfrak{X}) /\left(H^{2}(\mathfrak{X}, \mathbb{Z}) / \boldsymbol{\Lambda}^{\star}\right)\right] \times \mathbb{C}_{z}$. The quantum connection is a meromorphic flat partial connection on this vector bundle over the formal neighbourhood of the origin in $\mathcal{M}_{\mathrm{A}, \mathbb{T}}(\mathfrak{X})$ (times $\mathbb{C}_{z}$ ); it is given by

$$
\nabla=d+z^{-1} \sum_{i=0}^{s}\left(\phi_{i} \star_{\tau}\right) d \tau^{i}
$$

This connection is partial in the sense that it is defined only in the $\tau$-direction and not in the $z$-direction or in the direction of equivariant parameters (the first term $d$ means the relative differential over Spec $R_{\mathbb{T}}[z]$ ). The connection has simple poles along $z=0$ and has logarithmic singularities along the toric boundary of Spec $\mathbb{C}\left[\boldsymbol{\Lambda}_{+}\right]$. Note that $d \tau^{i}$ with $1 \leq i \leq r$ defines a logarithmic 1-form on Spec $\mathbb{C}\left[\boldsymbol{\Lambda}_{+}\right]$. In a more formal language, the module

$$
\left.H_{\mathrm{CR}, \mathbb{T}}^{*}(\mathfrak{X})[z] \llbracket \boldsymbol{\Lambda}_{+} \rrbracket \llbracket \tau^{\prime} \rrbracket:=H_{\mathrm{CR}, \mathbb{T}}^{*}(\mathfrak{X}) \otimes_{R_{\mathbb{T}}} R_{\mathbb{T}}[z] \llbracket \boldsymbol{\Lambda}_{+} \rrbracket \llbracket\left[\tau^{0}, \tau^{r+1}, \ldots, \tau^{s}\right]\right]
$$


(which we regard as the module of sections of the bundle (2.6) over the formal neighbourhood of the origin in $\left.\mathcal{M}_{\mathrm{A}, \mathbb{T}}(\mathfrak{X})\right)$ is equipped with $s+1$ operators $\nabla_{\frac{\partial}{\partial \tau^{i}}}=\frac{\partial}{\partial \tau^{i}}+z^{-1} \phi_{i} \star_{\tau}, i=0,1, \ldots, s$ :

$$
\nabla_{\frac{\partial}{\partial \tau^{i}}}: H_{\mathrm{CR}, \mathbb{T}}^{*}(\mathfrak{X})[z] \llbracket \boldsymbol{\Lambda}_{+} \rrbracket \llbracket \tau^{\prime} \rrbracket \rightarrow z^{-1} H_{\mathrm{CR}, \mathbb{T}}^{*}(\mathfrak{X})[z] \llbracket \boldsymbol{\Lambda}_{+} \rrbracket \llbracket \tau^{\prime} \rrbracket
$$

that commute each other: $\left[\nabla_{\frac{\partial}{\partial \tau^{i}}}, \nabla_{\frac{\partial}{\partial \tau^{j}}}\right]=0$.

Let $\left\{\chi_{1}, \ldots, \chi_{k}\right\}$ denote a basis of $H_{\mathbb{T}}^{2}(\mathrm{pt}, \mathbb{C})$ so that $R_{\mathbb{T}}=\mathbb{C}\left[\chi_{1}, \ldots, \chi_{k}\right]$. For $\xi \in H^{2}(\mathfrak{X}, \mathbb{C})$, we write $\xi q \frac{\partial}{\partial q}$ for the derivation of $\mathbb{C} \llbracket \boldsymbol{\Lambda}_{+} \rrbracket$ given by $\left(\xi q \frac{\partial}{\partial q}\right) q^{d}=(\xi \cdot d) q^{d}$. The Euler vector field $\mathcal{E}$ is the following derivation of $R_{\mathbb{T}} \llbracket \Lambda_{+} \rrbracket \llbracket \tau^{\prime} \rrbracket$

$$
\mathcal{E}=c_{1}(\mathfrak{X}) q \frac{\partial}{\partial q}+\sum_{i \in\{0, r+1, \ldots, s\}}\left(1-\frac{\operatorname{deg} \phi_{i}}{2}\right) \tau^{i} \frac{\partial}{\partial \tau^{i}}+\sum_{i=1}^{k} \chi_{i} \frac{\partial}{\partial \chi_{i}} .
$$

The grading operator $\operatorname{Gr} \in \operatorname{End}_{\mathbb{C}}\left(H_{\mathrm{CR}, \mathbb{T}}(\mathfrak{X})[z] \llbracket \boldsymbol{\Lambda}_{+} \rrbracket \llbracket \tau^{\prime} \rrbracket\right)$ is defined to be

$$
\operatorname{Gr}\left(f\left(q, \tau^{\prime}, \chi, z\right) \phi_{i}\right)=\left(\left(\mathcal{E}+z \frac{\partial}{\partial z}\right) f\left(q, \tau^{\prime}, \chi, z\right)\right) \phi_{i}+\frac{\operatorname{deg} \phi_{i}}{2} f\left(q, \tau^{\prime}, \chi, z\right) \phi_{i},
$$

where $f\left(q, \tau^{\prime}, \chi, z\right) \in R_{\mathbb{T}}[z] \llbracket \boldsymbol{\Lambda}_{+} \rrbracket \llbracket \tau^{\prime} \rrbracket$. This is compatible with the quantum connection in the sense that

$$
\left[\nabla_{\frac{\partial}{\partial \tau^{i}}}, \mathrm{Gr}\right]=\left(1-\frac{\operatorname{deg} \phi_{i}}{2}\right) \nabla_{\frac{\partial}{\partial \tau^{i}}}\left(=\nabla_{\left[\frac{\partial}{\partial \tau^{i}}, \mathcal{E}\right]}\right) .
$$

Let $P$ denote the pairing between the fibres of the bundle (2.6) at $\left(q, \tau^{\prime}, \chi,-z\right)$ and at $\left(q, \tau^{\prime}, \chi, z\right)$ induced by the orbifold Poincaré pairing. This gives the following $R_{\mathbb{T}} \llbracket \Lambda_{+} \rrbracket \llbracket \tau^{\prime} \rrbracket$-bilinear pairing on the module $H_{\mathrm{CR}, \mathbb{T}}^{*}(\mathfrak{X})[z] \llbracket \boldsymbol{\Lambda}_{+} \rrbracket \llbracket \tau^{\prime} \rrbracket$ :

$$
P(\alpha, \beta):=(\alpha(-z), \beta(z)) \in S_{\mathbb{T}}[z] \llbracket \Lambda_{+} \rrbracket \llbracket \tau^{\prime} \rrbracket,
$$

where $(\cdot, \cdot)$ denotes the orbifold Poincaré pairing $(2.2)$. The pairing $P$ satisfies

$$
\begin{aligned}
& P\left(f\left(q, \tau^{\prime}, \chi,-z\right) \alpha, \beta\right)=P\left(\alpha, f\left(q, \tau^{\prime}, \chi, z\right) \beta\right)=f\left(q, \tau^{\prime}, \chi, z\right) P(\alpha, \beta), \\
& P(\beta, \alpha)=\left.P(\alpha, \beta)\right|_{z \rightarrow-z}
\end{aligned}
$$

for $f\left(q, \tau^{\prime}, \chi, z\right) \in R_{\mathbb{T}}[z] \llbracket \Lambda_{+} \rrbracket \llbracket \tau^{\prime} \rrbracket$ and the compatibility equations with $\nabla$ and $\mathcal{E}$ :

$$
\begin{aligned}
& \frac{\partial}{\partial \tau^{i}} P(\alpha, \beta)=P\left(\widetilde{\nabla}_{\frac{\partial}{\partial \tau^{i}}} \alpha, \beta\right)+P\left(\alpha, \nabla_{\frac{\partial}{\partial \tau^{i}}} \beta\right), \\
& \left(z \frac{\partial}{\partial z}+\mathcal{E}\right) P(\alpha, \beta)=P(\operatorname{Gr} \alpha, \beta)+P(\alpha, \operatorname{Gr} \beta)-(\operatorname{dim} \mathfrak{X}) P(\alpha, \beta),
\end{aligned}
$$

where $\widetilde{\nabla}=\left.\nabla\right|_{z \rightarrow-z}$. The structures $\nabla$, Gr, $P$ are equivariant with respect to the Galois symmetry of $H^{2}(\mathfrak{X}, \mathbb{Z}) / \boldsymbol{\Lambda}^{\star}$. We call the $\left(H^{2}(\mathfrak{X}, \mathbb{Z}) / \boldsymbol{\Lambda}^{\star}\right)$-equivariant quadruple

$$
\mathrm{QDM}_{\mathbb{T}}(\mathfrak{X}):=\left(H_{\mathrm{CR}, \mathbb{T}}^{*}(\mathfrak{X})[z] \llbracket \boldsymbol{\Lambda}_{+} \rrbracket \llbracket \tau^{\prime} \rrbracket, \nabla, \mathrm{Gr}, P\right)
$$

the $\mathbb{T}$-equivariant quantum D-module of $\mathfrak{X}$.

Remark 2.6. The flat connection $\nabla$ defines on the space $H_{\mathrm{CR}, \mathbb{T}}^{*}(\mathfrak{X})[z] \llbracket \Lambda_{+} \rrbracket \llbracket \tau^{\prime} \rrbracket$ the structure of a module over the ring of differential operators

$$
R_{\mathbb{T}}[z] \llbracket \boldsymbol{\Lambda}_{+} \rrbracket \llbracket \tau^{\prime} \rrbracket\left\langle z \frac{\partial}{\partial \tau^{0}}, z \frac{\partial}{\partial \tau^{1}}, \ldots, z \frac{\partial}{\partial \tau^{s}}\right\rangle,
$$

where $z \frac{\partial}{\partial \tau^{i}}$ acts by the connection $z \nabla_{\frac{\partial}{\partial \tau^{i}}}$. This is often called a $z$-connection [94]. 
The non-equivariant quantum D-module is the restriction of the equivariant one to the origin $0 \in \operatorname{Spec} R_{\mathbb{T}}=$ Lie $\mathbb{T}$. It is a quadruple

$$
\operatorname{QDM}(\mathfrak{X}):=\left(H_{\mathrm{CR}}^{*}(\mathfrak{X})[z] \llbracket \boldsymbol{\Lambda}_{+} \rrbracket \llbracket \tau^{\prime} \rrbracket, \nabla, \mathrm{Gr}, P\right),
$$

where the pairing $P$ is defined only when $\mathfrak{X}$ is proper. In the non-equivariant case, we can define the connection in the $z$-direction by

$$
\nabla_{z \frac{\partial}{\partial z}}:=\mathrm{Gr}-\nabla_{\mathcal{E}}-\frac{\operatorname{dim} \mathfrak{X}}{2}=z \frac{\partial}{\partial z}-\frac{1}{z}\left(E \star_{\tau}\right)+\mu
$$

and this preserves the pairing $P$. Here $\mathcal{E}$ denotes the non-equivariant limit of (2.7) (and thus does not contain the term $\left.\sum_{i=1}^{n} \chi_{i} \frac{\partial}{\partial \chi_{i}}\right)$ and

$$
E=c_{1}(\mathfrak{X})+\sum_{i \in\{0, r+1, \ldots, s\}}\left(1-\frac{1}{2} \operatorname{deg} \phi_{i}\right) \tau^{i} \phi_{i}
$$

is the section of $\mathrm{QDM}(\mathfrak{X})$ corresponding to $\mathcal{E}$ and $\mu \in \operatorname{End}\left(H_{\mathrm{CR}}^{*}(\mathfrak{X})\right)$ is the endomorphism given by $\mu\left(\phi_{i}\right)=\left(\frac{1}{2} \operatorname{deg} \phi_{i}-\frac{1}{2} \operatorname{dim} \mathfrak{X}\right) \phi_{i}$.

\section{$2.4 \widehat{\Gamma}$-integral structure}

The $\widehat{\Gamma}$-integral structure [62, Section 2.4], [70, Proposition 3.1] is an integral lattice in the space of flat sections of the quantum D-module. The integral lattice is identified with the topological $K$-group of $\mathfrak{X}$. We review its definition only in the non-equivariant case (see [31, Section 3] for the equivariant $\widehat{\Gamma}$-integral structure).

For simplicity, we assume that the quantum product $\star_{\tau}$ is convergent in a neighbourhood of the large radius limit point. Then the quantum connection is also analytic in the same neighbourhood; it has no singularities on the intersection of the open subset $\left[H_{\mathrm{CR}}^{*}(\mathfrak{X}) / H^{2}(\mathfrak{X}, \mathbb{Z})\right]$ $\subset\left[\mathcal{M}_{\mathrm{A}}(\mathfrak{X}) /\left(H^{2}(\mathfrak{X}, \mathbb{Z}) / \boldsymbol{\Lambda}^{\star}\right)\right]$ and the convergence domain of $\star_{\tau}$. Introduce the following $\operatorname{End}\left(H_{\mathrm{CR}}^{*}(\mathfrak{X})\right)$-valued function:

$$
L(\tau, z) \phi_{i}=e^{-\sigma / z} \phi_{i}+\sum_{k=0}^{s} \sum_{\substack{n \geq 0, d \in \boldsymbol{\Lambda}_{+} \\(n, d) \neq(0,0)}}\left\langle\phi_{k}, \tau^{\prime}, \ldots, \tau^{\prime}, \frac{e^{-\sigma / z} \phi_{i}}{-z-\psi}\right\rangle_{0, l+2, d} \frac{e^{\sigma \cdot d}}{l !} \phi^{k},
$$

where we write $\tau=\sigma+\tau^{\prime} \in H_{\mathrm{CR}}^{*}(\mathfrak{X}), \sigma=\sum_{i=1}^{r} \tau^{i} \phi_{i} \in H^{2}(\mathfrak{X}), \tau^{\prime}=\tau^{0} \phi_{0}+\sum_{i=r+1}^{s} \tau^{i} \phi_{i}$ as before, $\left\{\phi^{k}\right\}$ is the dual basis of $\left\{\phi_{k}\right\}$ with respect to the orbifold Poincaré pairing, and $1 /(-z-\psi)$ should be expanded in the series $\sum_{k=0}^{\infty}(-z)^{-k-1} \psi^{k}$. We also set

$$
z^{-\mu} z^{c_{1}(\mathfrak{X})}:=e^{-\mu \log z} e^{c_{1}(\mathfrak{X}) \log z} .
$$

Then by [62, Proposition 2.4], $L(\tau, z) z^{-\mu} z^{c_{1}(\mathfrak{X})} \phi_{i}, i=0, \ldots, s$ form a basis of (multi-valued) $\nabla$ flat sections (which are flat also in the $z$-direction with respect to $(2.11)$ ), i.e., $L(\tau, z) z^{-\mu} z^{c_{1}(\mathfrak{X})}$ is a fundamental solution of the quantum connection.

We introduce the Chern character and the $\widehat{\Gamma}$-class for a smooth DM stack $\mathfrak{X}$. Let $\pi: I \mathfrak{X} \rightarrow \mathfrak{X}$ denote the natural projection. Recall the decomposition $I \mathfrak{X}=\bigsqcup_{v \in \operatorname{Box}} \mathfrak{X}_{v}$ into twisted sectors. For 
an orbi-vector bundle $V$ on $\mathfrak{X}$, the stabilizer along $\mathfrak{X}_{v}$ acts on $\left.\left(\pi^{*} V\right)\right|_{\mathfrak{X}_{v}}$ and decomposes it into the sum of eigenbundles

$$
\left.\pi^{*} V\right|_{\mathfrak{X}_{v}}=\bigoplus_{0 \leq f<1} V_{v, f}
$$

where the stabilizer acts on $V_{v, f}$ by $\exp (2 \pi \mathbf{i} f)$. The Chern character of $V$ is defined to be

$$
\widetilde{\operatorname{ch}}(V)=\bigoplus_{v \in \operatorname{Box}} \sum_{0 \leq f<1} e^{2 \pi \mathbf{i} f} \operatorname{ch}\left(V_{v, f}\right) \in H^{*}(I \mathfrak{X}) .
$$

Consider now the tangent bundle $V=T \mathfrak{X}$ and let $\delta_{v, f, j}, j=1, \ldots, \operatorname{rank}\left(V_{v, f}\right)$ denote the Chern roots of $T \mathfrak{X}_{v, f}$. The $\widehat{\Gamma}$-class of $\mathfrak{X}$ is defined to be

$$
\widehat{\Gamma}_{\mathfrak{X}}=\bigoplus_{v \in \text { Box }} \prod_{0 \leq f<1} \prod_{j=1}^{\operatorname{rank}\left(T \mathfrak{X}_{v, f}\right)} \Gamma\left(1-f+\delta_{v, f, j}\right) \in H^{*}(I \mathfrak{X})
$$

where in the right-hand side we expand the Euler $\Gamma$-function $\Gamma(z)$ in series at $z=1-f$. This is an algebraic cohomology class defined over transcendental numbers.

Definition 2.7 ([62, Definition 2.9]). Let $K(\mathfrak{X})$ denote the Grothendieck group of topological orbi-vector bundles on $\mathfrak{X}$. For $V \in K(\mathfrak{X})$, we define a (multi-valued) flat $\operatorname{section} \mathfrak{s}_{V}(\tau, z)$ of the quantum D-module by

$$
\mathfrak{s}_{V}(\tau, z)=\frac{1}{(2 \pi)^{\operatorname{dim} \mathfrak{X} / 2}} L(\tau, z) z^{-\mu} z^{c_{1}(\mathfrak{X})}\left(\widehat{\Gamma}_{\mathfrak{X}} \cup(2 \pi \mathbf{i})^{\operatorname{deg}_{0} / 2} \operatorname{inv}^{*} \widetilde{\operatorname{ch}}(V)\right),
$$

where $\operatorname{deg}_{0}$ denotes the grading operator on $H^{*}(I \mathfrak{X})$ without age shift, i.e., $\operatorname{deg}_{0}(\alpha)=p \alpha$ for $\alpha \in H^{p}(I \mathfrak{X})$. The map $V \mapsto \mathfrak{s}_{V}$ defines an integral lattice in the space of flat sections, which we call the $\widehat{\Gamma}$-integral structure.

Important properties of the $\widehat{\Gamma}$-integral structure are as follows [62, Proposition 2.10]:

- it is monodromy-invariant around the large radius limit point: we have

$$
d g(\xi)^{-1} \mathfrak{s}_{V}(g(\xi)(\tau), z)=\mathfrak{s}_{V \otimes L_{\xi}}(\tau, z),
$$

where $L_{\xi}$ is the line bundle corresponding to $\xi \in H^{2}(\mathfrak{X}, \mathbb{Z})$; therefore it defines a $\mathbb{Z}$-local system underlying the quantum D-module;

- it intertwines the Euler pairing with the orbifold Poincaré pairing: if $V_{1}, V_{2}$ are holomorphic orbi-vector bundles on $\mathfrak{X}$, we have

$$
\chi\left(V_{1}, V_{2}\right)=\left(\mathfrak{s}_{V_{1}}\left(\tau, e^{-\pi \mathbf{i}} z\right), \mathfrak{s}_{V_{2}}(\tau, z)\right),
$$

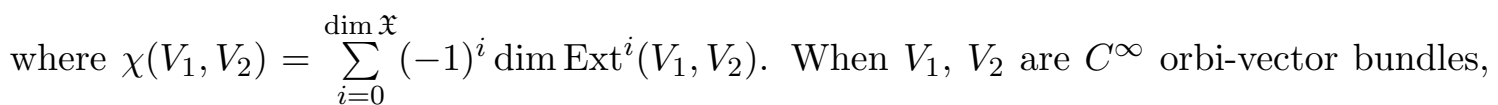
we can define $\chi\left(V_{1}, V_{2}\right)$ as the index of a Dirac operator on $V_{1}^{\vee} \otimes V_{2} \otimes \Omega_{\mathfrak{X}}^{0, \bullet}$ and this formula holds under mild assumptions, see [62, Remark 2.8]. Note that the right-hand side does not depend on $(\tau, z)$ since $\mathfrak{s}_{V_{1}}, \mathfrak{s}_{V_{2}}$ are flat sections.

Remark 2.8. The fundamental solution $L(\tau, z) z^{-\mu} z^{c_{1}(\mathfrak{X})}$ is multi-valued as a function of $(\tau, z) \in$ $\left[H_{\mathrm{CR}}^{*}(\mathfrak{X}) / H^{2}(\mathfrak{X} ; \mathbb{Z})\right] \times \mathbb{C}^{\times}$, but it has a standard determination when $\tau$ is the image of a real class in $H_{\mathrm{CR}}^{*}(\mathfrak{X} ; \mathbb{R})=H^{*}(I \mathfrak{X} ; \mathbb{R})$ sufficiently close to the large radius limit point and $z$ is positive real. We will sometimes use such a point as a base point. 


\section{Global Landau-Ginzburg mirrors of toric DM stacks}

In this section, we construct a global Landau-Ginzburg model (LG model) which is simultaneously mirror to several smooth toric DM stacks. For background materials on toric (DeligneMumford) stacks, we refer the reader to [14, 34, 42, 66, 67, 106].

\subsection{Toric data}

Throughout the paper, we fix the data $(\mathbf{N}, \Pi)$, where

- $\mathbf{N}$ is a finitely generated abelian group of rank $n$ (possibly having torsion) and

- $\Pi$ is a full-dimensional, convex, rational polyhedral cone in $\mathbf{N}_{\mathbb{R}}:=\mathbf{N} \otimes \mathbb{R}$.

We do not require that $\Pi$ is strictly convex; $\Pi$ will be the support of the fan of a smooth toric DM stack. To construct a mirror family of LG models, we choose a finite subset $S \subset \mathbf{N}$ such that

- $S$ generates the cone $\Pi$ over $\mathbb{R}_{\geq 0}$, i.e., $\Pi=\sum_{b \in S} \mathbb{R}_{\geq 0} b$.

The set $S$ specifies the set of monomials appearing in the LG model. We make the following technical assumption to ensure that the base of the mirror family has no generic stabilizers, that is, generic LG models have no automorphisms of diagonal symmetry.

Assumption 3.1. $S$ generates $\mathbf{N}$ as an abelian group, i.e., $\mathbf{N}=\mathbb{Z} S$.

This is not an essential restriction. In fact, if $S$ does not satisfy this assumption, we can first construct the mirror family by taking a bigger set $S^{\prime} \supset S$ satisfying the assumption, and then restrict the family to the subspace of the base corresponding to $S$ (then, the subspace has generic stabilizers).

Notation 3.2. We write $\mathbf{N}_{\text {tor }}$ for the torsion part of $\mathbf{N}$ and write $\overline{\mathbf{N}}=\mathbf{N} / \mathbf{N}_{\text {tor }}$ for the torsionfree quotient. For $v \in \mathbf{N}$, we write $\bar{v}$ for the image of $v$ in $\overline{\mathbf{N}}$. The subscripts $\mathbb{Q}, \mathbb{R}$, etc. means the tensor product with $\mathbb{Q}, \mathbb{R}$ over $\mathbb{Z}$, e.g., $\mathbf{N}_{\mathbb{R}}=\mathbf{N} \otimes_{\mathbb{Z}} \mathbb{R}$. For subsets $A \subset \mathbf{N}$ and $B \subset \mathbf{N}_{\mathbb{R}}$, we write $A \cap B:=\{a \in A: \bar{a} \in B\}$.

Definition 3.3. A stacky fan adapted to $S$ is a triple $\boldsymbol{\Sigma}=(\mathbf{N}, \Sigma, R)$ such that

(i) $\Sigma$ is a rational simplicial fan defined on the vector space $\mathbf{N}_{\mathbb{R}}$;

(ii) the support $|\Sigma|=\bigcup_{\sigma \in \Sigma} \sigma$ of the fan $\Sigma$ equals $\Pi=\sum_{b \in S} \mathbb{R}_{\geq 0} \bar{b}$;

(iii) there exists a strictly convex piecewise linear function $\eta: \Pi \rightarrow \mathbb{R}$ which is linear on each cone of $\Sigma$;

(iv) $R \subset S$ is a subset such that the map $R \ni b \mapsto \mathbb{R}_{\geq 0} \bar{b}$ gives a bijection between $R$ and the set $\Sigma(1)$ of one-dimensional cones of $\Sigma$.

The data $(\mathbf{N}, \Sigma, R)$ gives a stacky fan in the sense of Borisov, Chen and Smith [14]. We write $\mathfrak{X}_{\boldsymbol{\Sigma}}$ for the smooth toric DM stack (toric stack for short) defined by $\boldsymbol{\Sigma}$. We also set $R(\boldsymbol{\Sigma}):=R$ ("rays") and $G(\boldsymbol{\Sigma}):=S \backslash R$ ("ghost rays"). We denote by $\mathfrak{F a n}(S)$ the set of stacky fans adapted to $S$.

Remark 3.4. The above conditions (ii), (iii) imply that the corresponding toric DM stack $\mathfrak{X}_{\boldsymbol{\Sigma}}$ is semiprojective [34, Section 7.2], that is, the coarse moduli space $X_{\Sigma}$ of $\mathfrak{X}_{\Sigma}$ is projective over the affine variety $\operatorname{Spec} H^{0}\left(X_{\Sigma}, \mathcal{O}\right)$ and has a torus fixed point. Conversely, any semiprojective toric DM stack arises from some toric data $(\mathbf{N}, \Pi, S, \Sigma, R)$ in this section. 


\subsubsection{Toric DM stacks}

Let $\boldsymbol{\Sigma}=(\mathbf{N}, \Sigma, R) \in \mathfrak{F} \mathfrak{a n}(S)$ be a stacky fan adapted to $S$. When we start from the stacky fan $\boldsymbol{\Sigma}$, the set $G(\boldsymbol{\Sigma})=S \backslash R$ can be viewed as the data of an extended stacky fan in the sense of Jiang [68]. The extended stacky fan is given by the pair $(\boldsymbol{\Sigma}, G(\boldsymbol{\Sigma}))$ of the stacky fan $\boldsymbol{\Sigma}$ and the finite subset $G(\boldsymbol{\Sigma}) \subset \mathbf{N} \cap|\Sigma|$. We recall a definition of the smooth toric DM stack $\mathfrak{X}_{\boldsymbol{\Sigma}}[14,68]$ in terms of the extended stacky fan. The stacky fan $\boldsymbol{\Sigma}$ defines the fan sequence

$$
0 \longrightarrow \mathbb{L}^{\boldsymbol{\Sigma}} \longrightarrow \mathbb{Z}^{R(\boldsymbol{\Sigma})} \stackrel{\beta(\boldsymbol{\Sigma})}{\longrightarrow} \mathbf{N}
$$

where $\beta(\boldsymbol{\Sigma}): \mathbb{Z}^{R(\boldsymbol{\Sigma})} \rightarrow \mathbf{N}$ sends the basis $e_{b} \in \mathbb{Z}^{R(\boldsymbol{\Sigma})}$ corresponding to $b \in R(\boldsymbol{\Sigma})$ to $b \in \mathbf{N}$ and $\mathbb{L}^{\boldsymbol{\Sigma}}:=\operatorname{Ker}(\beta(\boldsymbol{\Sigma}))$. The dual sequence

$$
0 \longrightarrow \mathbf{M} \longrightarrow\left(\mathbb{Z}^{R(\boldsymbol{\Sigma})}\right)^{\star} \longrightarrow\left(\mathbb{L}^{\boldsymbol{\Sigma}}\right)^{\vee}
$$

is called the divisor sequence, where $\left(\mathbb{L}^{\boldsymbol{\Sigma}}\right)^{\vee}:=H^{1}\left(\operatorname{Cone}(\beta(\boldsymbol{\Sigma}))^{\star}\right)$ is the Gale dual of $\beta(\boldsymbol{\Sigma})$ (see [14]). The extended fan sequence is the sequence

$$
0 \longrightarrow \mathbb{L} \longrightarrow \mathbb{Z}^{S} \stackrel{\beta}{\longrightarrow} \mathbf{N} \longrightarrow 0
$$

where the map $\beta: \mathbb{Z}^{S} \rightarrow \mathbf{N}$ sends the basis $e_{b} \in \mathbb{Z}^{S}$ corresponding to $b \in S$ to $b \in \mathbf{N}$ and $\mathbb{L}:=\operatorname{Ker}(\beta)$. Note that $\beta$ is surjective by Assumption 3.1. The dual sequence

$$
0 \longrightarrow \mathbf{M} \longrightarrow\left(\mathbb{Z}^{S}\right)^{\star} \stackrel{D}{\longrightarrow} \mathbb{L}^{\star}
$$

is called the extended divisor sequence, where $\mathbf{M}:=\operatorname{Hom}(\mathbf{N}, \mathbb{Z})$ and $D:\left(\mathbb{Z}^{S}\right)^{\star} \rightarrow \mathbb{L}^{\star}$ is dual to $\mathbb{L} \rightarrow \mathbb{Z}^{S}$ and $\operatorname{Cok}(D) \cong \operatorname{Ext}^{1}(\mathbf{N}, \mathbb{Z})$. The torus $\mathbb{L}_{\mathbb{C}^{\times}}:=\mathbb{L} \otimes \mathbb{C}^{\times}$acts on $\mathbb{C}^{S}$ via the natural map $\mathbb{L}_{\mathbb{C}^{\times}} \rightarrow\left(\mathbb{C}^{\times}\right)^{S}$ induced by $\mathbb{L} \rightarrow \mathbb{Z}^{S}$. The toric stack $\mathfrak{X}_{\Sigma}$ is defined as a GIT quotient of $\mathbb{C}^{S}$ by the $\mathbb{L}_{\mathbb{C}^{\times} \text {-action. Set }}$

$$
\mathscr{S}_{\boldsymbol{\Sigma}}:=\{I \subset R(\boldsymbol{\Sigma}): \text { the cone spanned by } I \text { belongs to } \Sigma\}
$$

and define the open subset $U_{\boldsymbol{\Sigma}} \subset \mathbb{C}^{S}$ as

$$
U_{\Sigma}=\mathbb{C}^{S} \backslash \bigcup_{I \subset S, I \notin \mathscr{S}_{\Sigma}} \mathbb{C}^{S \backslash I}
$$

where we regard $\mathbb{C}^{I}$ with $I \subset S$ as a co-ordinate subspace of $\mathbb{C}^{S}$ (we set $\mathbb{C}^{\varnothing}=\{0\}$ ). Since every element of $\mathscr{S}_{\boldsymbol{\Sigma}}$ is contained in $R(\boldsymbol{\Sigma})$, we may also write

$$
U_{\boldsymbol{\Sigma}}=\left(\mathbb{C}^{R(\boldsymbol{\Sigma})} \backslash \bigcup_{I \subset R(\boldsymbol{\Sigma}), I \notin \mathscr{S}} \mathbb{C}^{R(\boldsymbol{\Sigma}) \backslash I}\right) \times\left(\mathbb{C}^{\times}\right)^{G(\boldsymbol{\Sigma})} .
$$

We define

$$
\mathfrak{X}_{\Sigma}:=\left[U_{\Sigma} / \mathbb{L}_{\mathbb{C}^{\times}}\right] .
$$

Since $\mathbb{L}_{\mathbb{C}^{\times}}$acts on $U_{\boldsymbol{\Sigma}}$ with at most finite stabilizers, $\mathfrak{X}_{\boldsymbol{\Sigma}}$ is a smooth DM stack. The toric stack $\mathfrak{X}_{\boldsymbol{\Sigma}}$ depends only on $\boldsymbol{\Sigma}=(\mathbf{N}, \Sigma, R)$ and does not depend on the choice of the extension $G(\boldsymbol{\Sigma})=S \backslash R$ (see [68]). The coarse moduli space $X_{\Sigma}$ of $\mathfrak{X}_{\boldsymbol{\Sigma}}$ is the toric variety associated with the fan $\Sigma$. The $\left(\mathbb{C}^{\times}\right)^{S}$-action on $U_{\boldsymbol{\Sigma}}$ induces the $\mathbb{T}$-action on $\mathfrak{X}_{\boldsymbol{\Sigma}}$, where $\mathbb{T}$ is the torus

$$
\mathbb{T}:=\left(\mathbb{C}^{\times}\right)^{S} / \operatorname{Im}\left(\mathbb{L}_{\mathbb{C}^{\times}} \rightarrow\left(\mathbb{C}^{\times}\right)^{S}\right) \cong \mathbf{N} \otimes \mathbb{C}^{\times} .
$$




\subsubsection{Picard group and second (co)homology}

A character $\xi \in \operatorname{Hom}\left(\mathbb{L}_{\mathbb{C}^{\times}}, \mathbb{C}^{\times}\right)=\mathbb{L}^{\star}$ of $\mathbb{L}_{\mathbb{C}^{\times}}$defines a line bundle $L_{\xi}=\left[\left(U_{\boldsymbol{\Sigma}} \times \mathbb{C}\right) / \mathbb{L}_{\mathbb{C}^{\times}}\right]$over $\mathfrak{X}_{\boldsymbol{\Sigma}}$, where $\mathbb{L}_{\mathbb{C}^{\times}}$acts on the second factor $\mathbb{C}$ by the character $\xi$. We denote by $D_{b}:=D\left(e_{b}^{\star}\right)$ the image of the standard basis $e_{b}^{\star} \in\left(\mathbb{Z}^{S}\right)^{\star}$ under $D$. We can see that $D_{b}$ with $b \in G(\boldsymbol{\Sigma})$ yields the trivial line bundle on $\mathfrak{X}_{\boldsymbol{\Sigma}}$, and the correspondence $\xi \mapsto L_{\xi}$ gives the identification

$$
\mathbb{L}^{\star} / \sum_{b \in G(\boldsymbol{\Sigma})} \mathbb{Z} D_{b} \cong \operatorname{Pic}\left(\mathfrak{X}_{\boldsymbol{\Sigma}}\right)
$$

This group is isomorphic to the Gale dual $\left(\mathbb{L}^{\boldsymbol{\Sigma}}\right)^{\vee}$ of $\beta(\boldsymbol{\Sigma})$ appearing in (3.2). The torsion free quotient of $\operatorname{Pic}\left(\mathfrak{X}_{\boldsymbol{\Sigma}}\right)$ can be identified with the ordinary dual $\left(\mathbb{L}^{\boldsymbol{\Sigma}}\right)^{\star}$. Moreover we have the identifications

$$
\begin{aligned}
& H^{2}\left(\mathfrak{X}_{\boldsymbol{\Sigma}}, \mathbb{Q}\right) \cong\left(\mathbb{L}_{\mathbb{Q}}^{\boldsymbol{\Sigma}}\right)^{\star}, \\
& H_{\mathbb{T}}^{2}\left(\mathfrak{X}_{\boldsymbol{\Sigma}}, \mathbb{Q}\right) \cong\left(\mathbb{Q}^{R(\boldsymbol{\Sigma})}\right)^{\star}, \\
& H_{\mathbb{T}}^{2}(\mathrm{pt}, \mathbb{Q}) \cong \mathbf{M}_{\mathbb{Q}},
\end{aligned}
$$

so that the divisor sequence (3.2) over $\mathbb{Q}$ is identified with

$$
0 \longrightarrow H_{\mathbb{T}}^{2}(\mathrm{pt}, \mathbb{Q}) \longrightarrow H_{\mathbb{T}}^{2}\left(\mathfrak{X}_{\Sigma}, \mathbb{Q}\right) \longrightarrow H^{2}\left(\mathfrak{X}_{\Sigma}, \mathbb{Q}\right) \longrightarrow 0 .
$$

We write $\bar{D}_{b}$ for the image of $D_{b} \in \mathbb{L}^{\star}$ in $\left(\mathbb{L}_{\mathbb{Q}}^{\Sigma}\right)^{\star} \cong H^{2}\left(\mathfrak{X}_{\Sigma}, \mathbb{Q}\right)$. This is the class of a toric divisor. We also write

$$
R_{\mathbb{T}}:=H_{\mathbb{T}}^{*}(\mathrm{pt}, \mathbb{C}) \cong \operatorname{Sym}\left(\mathbf{M}_{\mathbb{C}}\right)
$$

for the $\mathbb{T}$-equivariant cohomology of a point.

\subsubsection{Orbifold cohomology}

For a cone $\sigma$ of $\Sigma$, we introduce $\operatorname{Box}(\sigma) \subset \mathbf{N}$ as

$$
\operatorname{Box}(\sigma)=\left\{v \in \mathbf{N}: \bar{v} \text { is of the form } \sum_{b \in \sigma \cap R(\boldsymbol{\Sigma})} c_{b} \bar{b} \text { for some } c_{b} \in[0,1)\right\}
$$

and set $\operatorname{Box}(\boldsymbol{\Sigma})=\bigcup_{\sigma \in \Sigma} \operatorname{Box}(\sigma)$. The set $\operatorname{Box}(\boldsymbol{\Sigma})$ parametrizes connected components of the inertia stack $I \mathfrak{X}_{\boldsymbol{\Sigma}}$ [14]. We write $\mathfrak{X}_{\boldsymbol{\Sigma}, v}$ for the component of $I \mathfrak{X}_{\boldsymbol{\Sigma}}$ corresponding to $v \in \operatorname{Box}(\boldsymbol{\Sigma})$. The age of a box element $v \in \operatorname{Box}(\sigma)$ is given by age $(v):=\sum_{b \in R(\boldsymbol{\Sigma}) \cap \sigma} c_{b}$ when we write $\bar{v}=\sum_{b \in R(\boldsymbol{\Sigma}) \cap \sigma} c_{b} \bar{b}$ with $c_{b} \geq 0$. The orbifold cohomology $H_{\mathrm{CR}}^{*}\left(\mathfrak{X}_{\boldsymbol{\Sigma}}\right)$ of Chen and Ruan [24] is given by (as a graded vector space):

$$
H_{\mathrm{CR}}^{*}\left(\mathfrak{X}_{\boldsymbol{\Sigma}}\right)=\bigoplus_{v \in \operatorname{Box}(\boldsymbol{\Sigma})} H^{*-2 \operatorname{age}(v)}\left(\mathfrak{X}_{\boldsymbol{\Sigma}, v}, \mathbb{C}\right) .
$$

As a ring, it is generated by the fundamental classes $\mathbf{1}_{v}$ on $I \mathfrak{X}_{\boldsymbol{\Sigma}, v}$ with $v \in \operatorname{Box}(\boldsymbol{\Sigma})$ and the toric divisor classes $\bar{D}_{b} \in H^{2}\left(\mathfrak{X}_{\boldsymbol{\Sigma}}, \mathbb{Q}\right)$ with $b \in R(\boldsymbol{\Sigma})$. Here we regard $\bar{D}_{b}$ as a class supported on the untwisted sector $\mathfrak{X}_{\boldsymbol{\Sigma}, 0}=\mathfrak{X}_{\boldsymbol{\Sigma}}$. The $\mathbb{T}$-equivariant orbifold cohomology $H_{\mathrm{CR}, \mathbb{T}}^{*}\left(\mathfrak{X}_{\boldsymbol{\Sigma}}\right)$ is defined by replacing each factor in the right-hand side of (3.7) with the $\mathbb{T}$-equivariant cohomology $H_{\mathbb{T}}^{*-2}$ age $(v)\left(\mathfrak{X}_{\boldsymbol{\Sigma}, v}\right)$. 


\subsection{Landau-Ginzburg model}

We construct a global family of mirror LG models which are simultaneously mirror to all the toric stacks $\mathfrak{X}_{\boldsymbol{\Sigma}}$ with $\boldsymbol{\Sigma} \in \mathfrak{F a n}(S)$.

The uncompactified $L G$ model $[47,58,62]$ is the family of tori

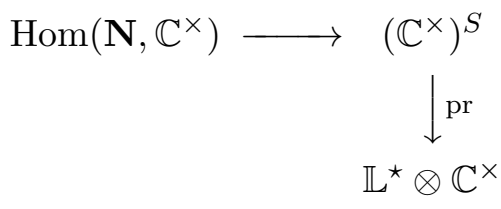

obtained from the extended fan sequence (3.3) by applying $\operatorname{Hom}\left(-, \mathbb{C}^{\times}\right)$, together with the function $F:\left(\mathbb{C}^{\times}\right)^{S} \rightarrow \mathbb{C}$

$$
F=\sum_{b \in S} u_{b}
$$

where $u_{b}$ denotes the $\mathbb{C}^{\times}$-valued co-ordinate on $\left(\mathbb{C}^{\times}\right)^{S}$ given by the projection to the $b$ th factor.

We shall partially compactify this family to include all the large radius limit points of $\mathfrak{X}_{\boldsymbol{\Sigma}}$ with $\boldsymbol{\Sigma} \in \mathfrak{F a n}(S)$. We construct partial compactifications of $\left(\mathbb{C}^{\times}\right)^{S}$ and $\mathbb{L}^{\star} \otimes \mathbb{C}^{\times}$as possibly singular toric DM stacks in the sense of Tyomkin [106]. According to Tyomkin [106, Section 4.1], a singular toric DM stack can be described by toric stacky data $(L, \Xi, 7)$ such that:

- $L$ is a finitely generated free abelian group;

- $\Xi$ is a (not necessarily simplicial) rational fan on $L_{\mathbb{R}}=L \otimes \mathbb{R}$;

- $\neg \subset|\Xi|$ is a subset in the support $|\Xi|$ of $\Xi$ such that for each cone $\sigma \in \Xi$, there exists a finite index sublattice $L(\sigma) \subset L$ such that $\neg \cap \sigma=L(\sigma) \cap \sigma$. We call 7 the integral structure of the toric stacky data.

Note that this is a generalization of a stacky fan $(\mathbf{N}, \Sigma, R)$ of Borisov, Chen and Smith [14] when the group $\mathbf{N}$ has no torsion. For a given stacky fan $(\mathbf{N}, \Sigma, R)$ with free $\mathbf{N}$, we can assign a toric stacky data $(\mathbf{N}, \Sigma\urcorner$,$) by taking 7$ to be the union of the monoids $\sigma_{\mathbb{Z}}=\mathbb{Z}_{\geq 0}(R \cap \sigma)$ for all $\sigma \in \Sigma$. Tyomkin constructed a singular toric DM stack from $(L, \Xi\rceil$,$) by gluing affine charts; its coarse$ moduli space is the toric variety $X_{\Xi}$ associated with the fan $\Xi$. We refer the reader to [106, Section 4.1] for the details (the construction of the affine charts in our case will be reviewed in Section 3.4).

Notation 3.5. For $c=\left(c_{b}\right)_{b \in S} \in\left(\mathbb{R}^{S}\right)^{\star}$, we define a convex piecewise linear function $\eta_{c}: \Pi \rightarrow \mathbb{R}$ by

$$
\eta_{c}(v):=\max \left\{\varphi(v): \varphi \in \mathbf{M}_{\mathbb{R}}=\operatorname{Hom}\left(\mathbf{N}_{\mathbb{R}}, \mathbb{R}\right), \varphi(\bar{b}) \leq c_{b}(\forall b \in S)\right\}
$$

This is well-defined when there exists $\varphi \in \mathbf{M}_{\mathbb{R}}$ such that $\varphi(\bar{b}) \leq c_{b}$ for all $b \in S$; in particular if $c_{b} \geq 0$ for all $b \in S$. The graph of $\eta_{c}$ is the union of "lower faces" of the convex cone in $\mathbf{N}_{\mathbb{R}} \oplus \mathbb{R}$ generated by $\left(\bar{b}, c_{b}\right), b \in S$ and $(0,1)$.

For a stacky fan $\boldsymbol{\Sigma}=(\mathbf{N}, \Sigma, R)$ adapted to $S$, we define a full-dimensional strictly-convex cone $\mathrm{CPL}_{+}(\boldsymbol{\Sigma}) \subset\left(\mathbb{R}^{S}\right)^{\star}$ by

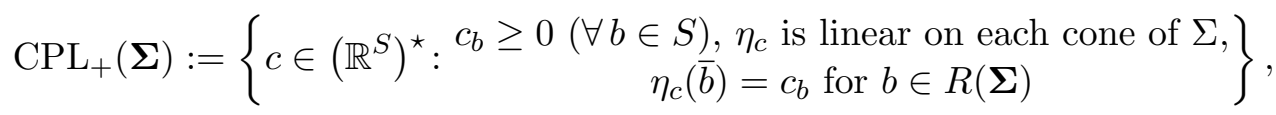


where CPL stands for "convex piecewise linear" (notation borrowed from [86]) and the subscript + means non-negative. Note that $\eta_{c}$ in the definition is determined only by $\left\{c_{b}: b \in R(\boldsymbol{\Sigma})\right\}$. Note also that $c_{b} \geq \eta_{c}(\bar{b})$ for $b \in G(\boldsymbol{\Sigma})$. We define the integral structure $\widetilde{\neg} \subset\left(\mathbb{R}^{S}\right)^{\star}$ as

$$
\widetilde{\urcorner}:=\left\{c \in\left(\mathbb{Z}^{S}\right)^{\star}: c_{b} \geq 0(\forall b \in S) \text {, and } \eta_{c}(\bar{b}) \in \mathbb{Z}(\forall b \in \mathbf{N} \cap \Pi)\right\} .
$$

Note that $\widetilde{7} \cap \mathrm{CPL}_{+}(\boldsymbol{\Sigma})$ equals the intersection of the following sublattice of $\left(\mathbb{Z}^{S}\right)^{\star}$

$$
\mathrm{PL}_{\mathbb{Z}}(\boldsymbol{\Sigma}):=\left\{c \in\left(\mathbb{Z}^{S}\right)^{\star}: \forall \sigma \in \Sigma, \exists m_{\sigma} \in \mathbf{M} \text { s.t. } m_{\sigma}(b)=c_{b}(\forall b \in R(\boldsymbol{\Sigma}) \cap \sigma)\right\}
$$

with the cone $\mathrm{CPL}_{+}(\boldsymbol{\Sigma})$. We also define

$$
\operatorname{cpl}(\boldsymbol{\Sigma}):=D\left(\mathrm{CPL}_{+}(\boldsymbol{\Sigma})\right), \quad \boldsymbol{\top}:=D(\widetilde{\boldsymbol{\top}}),
$$

where $D:\left(\mathbb{R}^{S}\right)^{\star} \rightarrow \mathbb{L}_{\mathbb{R}}^{\star}$ is the map appearing in the extended divisor sequence (3.4). The cone $\operatorname{cpl}(\boldsymbol{\Sigma})$ consists of convex piecewise linear functions (with respect to $\boldsymbol{\Sigma}$ ) modulo linear functions. It is easy to check that $\mathrm{CPL}_{+}(\boldsymbol{\Sigma})=D^{-1}(\operatorname{cpl}(\boldsymbol{\Sigma})) \cap\left(\mathbb{R}_{\geq 0}\right)^{S}$ and that $7 \cap \operatorname{cpl}(\boldsymbol{\Sigma})$ is the intersection of the finite index sublattice $\operatorname{pl}_{\mathbb{Z}}(\boldsymbol{\Sigma}):=\mathrm{PL}_{\mathbb{Z}}(\boldsymbol{\Sigma}) / \mathbf{M}$ of $\mathbb{L}^{\star}$ with the cone $\operatorname{cpl}(\boldsymbol{\Sigma})$.

Definition 3.6 (partially compactified LG model). Let $\widetilde{\Xi}$ be the fan in $\left(\mathbb{R}^{S}\right)^{\star}$ consisting of the maximal cones $\mathrm{CPL}_{+}(\boldsymbol{\Sigma})$ with $\boldsymbol{\Sigma} \in \mathfrak{F a n}(S)$ and their faces. Let $\Xi$ be the fan in $\mathbb{L}_{\mathbb{R}}^{\star}$ consisting of the maximal cones $\operatorname{cpl}(\boldsymbol{\Sigma})$ with $\boldsymbol{\Sigma} \in \mathfrak{F a n}(S)$ and their faces.

1. Define $\mathcal{Y}$ to be the singular toric DM stack corresponding to the toric stacky data $\left(\left(\mathbb{Z}^{S}\right)^{\star}, \widetilde{\Xi}, \widetilde{\urcorner}\right)$. We call $\mathcal{Y}$ the total space of the LG model.

2. Define $\mathcal{M}$ to be the singular toric DM stack corresponding to the toric stacky data $\left(\mathbb{L}^{\star}, \Xi, 7\right)$. We call $\mathcal{M}$ the secondary toric stack.

3. The map $D:\left(\mathbb{Z}^{S}\right)^{\star} \rightarrow \mathbb{L}^{\star}$ defines a map between these toric stacky data, and thus induces a toric morphism pr: $\mathcal{Y} \rightarrow \mathcal{M}$. Let $u_{b}: \mathcal{Y} \rightarrow \mathbb{C}$ with $b \in S$ denote the regular function defined by $e_{b} \in \mathbb{Z}^{S}$, and define the LG potential to be $F=\sum_{b \in S} u_{b}$. We call the pair $(\operatorname{pr}: \mathcal{Y} \rightarrow \mathcal{M}, F)$ the (partially compactified) $L G$ model associated to $S$.

Definition 3.7. Each stacky fan $\boldsymbol{\Sigma} \in \mathfrak{F a n}(S)$ gives rise to a torus-fixed point $0_{\boldsymbol{\Sigma}}$ of the secondary toric stack $\mathcal{M}$. We call $0_{\boldsymbol{\Sigma}}$ the large radius limit point of $\mathfrak{X}_{\boldsymbol{\Sigma}}$.

\section{Remark 3.8.}

(1) The fan $\Xi$ on $\mathbb{L}_{\mathbb{R}}^{\star}$ defined by the cones $\operatorname{cpl}(\boldsymbol{\Sigma})$ is called the secondary fan or the GKZ fan after the work of Gelfand, Kapranov and Zelevinsky [45] (see also Oda-Park [86]). The fan $\widetilde{\Xi}$ gives a lift of the secondary fan $\Xi$ to $\left(\mathbb{R}^{S}\right)^{\star}$. The support of $\widetilde{\Xi}$ is the positive orthant $\left(\mathbb{R}_{\geq 0}\right)^{S}$, and therefore $\mathcal{Y}$ can be viewed as an iterated weighted blowup of $\mathbb{C}^{S}$. The fibre of the map $D:|\widetilde{\Xi}|=\left(\mathbb{R}_{\geq 0}\right)^{S} \rightarrow \mathbb{L}_{\mathbb{R}}^{\star}$ at an interior point $\omega$ of $\operatorname{cpl}(\boldsymbol{\Sigma}) \subset \mathbb{L}_{\mathbb{R}}^{\star}$ can be identified with the image of the moment map $\mu: X_{\Sigma} \rightarrow \mathbf{M}_{\mathbb{R}}$ of the $\mathbb{T}$-action on $X_{\Sigma}$ with respect to the reduced symplectic form associated with $\omega$. Therefore the fan $\widetilde{\Xi}$ can be viewed as the total space of the moment polytope fibration over the secondary fan $\Xi$.

(2) Diemer, Katzarkov and Kerr [36] introduced a closely related (but slightly different) compactification of the LG model in the case where $S$ lies in a hyperplane of integral distance one from the origin. In this case (i.e., when $S$ lies in a hyperplane of height one), our space $\mathcal{Y}$ can be obtained from their total Lafforgue stack [36] by contracting a divisor (the zero-section), at least on the level of coarse moduli spaces. Their secondary stack [36] and our secondary toric stack are the same on the level of coarse moduli spaces (the coarse moduli spaces are the toric variety defined by the fan $\Xi$ ), however it is not clear to the author if the stack structures are the same. 
Remark 3.9. Cones of $\widetilde{\Xi}, \Xi$ can be described more explicitly as follows. A possibly degenerate fan [86] on $\mathbf{N}_{\mathbb{R}}$ is a finite collection $\Sigma$ of convex (but not necessarily strictly convex) rational polyhedral cones in $\mathbf{N}_{\mathbb{R}}$ such that (1) if $\sigma \in \Sigma$ and $\tau$ is a face of $\sigma$, then $\tau \in \Sigma$; and (2) if $\sigma, \tau \in \Sigma$ then the intersection $\sigma \cap \tau$ is a common face of $\sigma$ and $\tau$. Let $\Sigma$ be a possibly degenerate fan on $\mathbf{N}_{\mathbb{R}}$. Each cone $\sigma \in \Sigma$ contains the linear subspace $V=\sigma \cap(-\sigma)$ as a face, and the linear subspace $V$ does not depend on $\sigma$. When $V=0, \Sigma$ is a fan in the usual sense. A spanning set [86] of $\Sigma$ is a finite subset $R^{\prime} \subset \mathbf{N}$ such that each cone $\sigma \in \Sigma$ is generated by a subset of $R^{\prime}$ over $\mathbb{R}_{\geq 0}$. Let $\left(\Sigma, R^{\prime}, \sigma\right)$ be a triple such that $\Sigma$ is a possibly degenerate fan on $\mathbf{N}_{\mathbb{R}}$ with support $|\Sigma|=\Pi$ which admits a strictly convex piecewise linear function $\eta: \Pi \rightarrow \mathbb{R}$ linear on each cone of $\Sigma, R^{\prime} \subset S$ is a spanning set of $\Sigma$, and $\sigma \in \Sigma$ is a cone. For such a triple, we define the cone $\mathrm{CPL}_{+}\left(\Sigma, R^{\prime}, \sigma\right) \subset\left(\mathbb{R}^{S}\right)^{\star}$ as

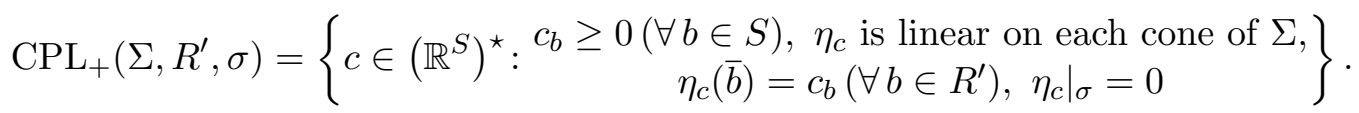

When $\boldsymbol{\Sigma}=(\mathbf{N}, \Sigma, R)$ is a stacky fan adapted to $S, R$ is a spanning set for $\Sigma$ and we have $\mathrm{CPL}_{+}(\boldsymbol{\Sigma})=\mathrm{CPL}_{+}(\Sigma, R,\{0\})$. Then the fan $\widetilde{\Xi}$ consists of the cones $\mathrm{CPL}_{+}\left(\Sigma, R^{\prime}, \sigma\right)$ and the fan $\Xi$ consists of the cones $\operatorname{cpl}\left(\Sigma, R^{\prime}\right)=D\left(\mathrm{CPL}_{+}\left(\Sigma, R^{\prime}, \sigma\right)\right.$ ) (which are independent of $\left.\sigma\right)$.

\subsection{Extended refined fan sequence and extended Mori cone}

The refined fan sequence [27] is an extension of the fan sequence (3.1) by a finite group. In this section we describe an extended version of the refined fan sequence for $\boldsymbol{\Sigma} \in \mathfrak{F a n}(S)$ (extended by ghost vectors $G(\boldsymbol{\Sigma})=S \backslash R(\boldsymbol{\Sigma})$ ). This will be used to describe a local chart of the global LG model (pr: $\mathcal{Y} \rightarrow \mathcal{M}, F)$.

Notation 3.10. Define a function $\Psi^{\Sigma}: \Pi \rightarrow\left(\mathbb{R}_{\geq 0}\right)^{S}$ as follows. For $v \in \Pi$, we take a cone $\sigma \in \Sigma$ containing $v$, and write $v=\sum_{b \in R(\boldsymbol{\Sigma}) \cap \sigma} c_{b} \bar{b}$. Then $\Psi^{\boldsymbol{\Sigma}}(v)=\left(\Psi_{b}^{\boldsymbol{\Sigma}}(v)\right)_{b \in S}$ is given by

$$
\Psi_{b}^{\Sigma}(v)= \begin{cases}c_{b}, & \text { if } b \in R(\boldsymbol{\Sigma}) \cap \sigma \\ 0, & \text { otherwise }\end{cases}
$$

The map $\Psi^{\boldsymbol{\Sigma}}$ gives a section of the $\operatorname{map} \beta:\left(\mathbb{R}_{\geq 0}\right)^{S} \rightarrow \Pi$, i.e., $\beta \circ \Psi^{\boldsymbol{\Sigma}}=\mathrm{id}_{\Pi}$.

We define $\mathbb{O}(\boldsymbol{\Sigma}) \subset \mathbb{Q}^{S} \oplus \mathbf{N}$ to be the subgroup:

$$
\mathbb{O}(\boldsymbol{\Sigma}):=\sum_{v \in \mathbf{N} \cap \Pi} \mathbb{Z}\left(\Psi^{\boldsymbol{\Sigma}}(v), v\right)+\sum_{b \in G(\boldsymbol{\Sigma})} \mathbb{Z}\left(e_{b}, b\right)
$$

and define $\boldsymbol{\Lambda}(\boldsymbol{\Sigma}) \subset \mathbb{L}_{\mathbb{Q}}$ to be

$$
\boldsymbol{\Lambda}(\boldsymbol{\Sigma}):=\left\{\lambda \in \mathbb{Q}^{S}:(\lambda, 0) \in \mathbb{O}(\boldsymbol{\Sigma})\right\} .
$$

Note that $\mathbb{O}(\boldsymbol{\Sigma})$ is contained in $\left\{(\lambda, v) \in \mathbb{Q}^{S} \oplus \mathbf{N}: \beta(\lambda)=\bar{v}\right\}$. These groups define the extended refined fan sequence:

$$
0 \longrightarrow \mathbf{\Lambda}(\boldsymbol{\Sigma}) \longrightarrow \mathbb{O}(\boldsymbol{\Sigma}) \longrightarrow \mathbf{N} \longrightarrow 0
$$

where the map $\mathbb{O}(\boldsymbol{\Sigma}) \rightarrow \mathbf{N}$ is given by the second projection. This is compatible with the extended fan sequence (3.3) under the inclusions $\mathbb{L} \subset \boldsymbol{\Lambda}(\boldsymbol{\Sigma}), \mathbb{Z}^{S} \subset \mathbb{O}(\boldsymbol{\Sigma})$, where the second inclusion sends $e_{b}$ to $\left(e_{b}, b\right)$. This sequence splits because the torsion part of $\mathbb{O}(\boldsymbol{\Sigma})$ is isomorphic 
to the torsion part $\mathbf{N}_{\text {tor }}$ of $\mathbf{N}$. The original refined fan sequence in [27] corresponds to the case where $G(\boldsymbol{\Sigma})=\varnothing$; it is the exact sequence

$$
0 \longrightarrow \Lambda^{\Sigma} \longrightarrow \mathbb{O}^{\Sigma} \longrightarrow \mathbf{N} \longrightarrow 0,
$$

where

$$
\begin{aligned}
& \mathbb{O}^{\boldsymbol{\Sigma}}:=\mathbb{O}(\boldsymbol{\Sigma}) \cap\left(\mathbb{Q}^{R(\boldsymbol{\Sigma})} \oplus \mathbf{N}\right)=\sum_{v \in \Pi \cap \mathbf{N}} \mathbb{Z}\left(\Psi^{\boldsymbol{\Sigma}}(v), v\right), \\
& \boldsymbol{\Lambda}^{\boldsymbol{\Sigma}}:=\boldsymbol{\Lambda}(\boldsymbol{\Sigma}) \cap \mathbb{Q}^{R(\boldsymbol{\Sigma})}=\left\{\lambda \in \mathbb{Q}^{R(\boldsymbol{\Sigma})}:(\lambda, 0) \in \mathbb{O}^{\boldsymbol{\Sigma}}\right\} .
\end{aligned}
$$

Note that $\boldsymbol{\Lambda}^{\boldsymbol{\Sigma}}$ is a lattice in $\mathbb{L}_{\mathbb{Q}}^{\boldsymbol{\Sigma}}=\mathbb{L}^{\boldsymbol{\Sigma}} \otimes \mathbb{Q}$, where $\mathbb{L}^{\boldsymbol{\Sigma}}$ is the lattice appearing in the fan sequence (3.1). For $b \in G(\boldsymbol{\Sigma})$, we define $\delta_{b}^{\boldsymbol{\Sigma}}:=e_{b}-\Psi^{\boldsymbol{\Sigma}}(b) \in \mathbb{Q}^{S}$. Then $\delta_{b}^{\boldsymbol{\Sigma}}$ lies in $\boldsymbol{\Lambda}(\boldsymbol{\Sigma})$ and we have the following decompositions:

$$
\mathbb{O}(\boldsymbol{\Sigma})=\mathbb{O}^{\boldsymbol{\Sigma}} \oplus \bigoplus_{b \in G(\boldsymbol{\Sigma})} \mathbb{Z} \delta_{b}^{\boldsymbol{\Sigma}}, \quad \boldsymbol{\Lambda}(\boldsymbol{\Sigma})=\boldsymbol{\Lambda}^{\boldsymbol{\Sigma}} \oplus \bigoplus_{b \in G(\boldsymbol{\Sigma})} \mathbb{Z} \delta_{b}^{\boldsymbol{\Sigma}}
$$

Remark 3.11 ([27, Section 2.4]). Under the identification $\mathbb{L}_{\mathbb{Q}}^{\boldsymbol{\Sigma}} \cong H_{2}\left(\mathfrak{X}_{\boldsymbol{\Sigma}}, \mathbb{Q}\right)$ in (3.6), the lattice $\Lambda^{\boldsymbol{\Sigma}} \subset \mathbb{L}_{\mathbb{Q}}^{\boldsymbol{\Sigma}}$ contains classes of all orbifold stable maps to $\mathfrak{X}_{\boldsymbol{\Sigma}}$. We also have an isomorphism $\left(\boldsymbol{\Lambda}^{\boldsymbol{\Sigma}}\right)^{\star} \cong \operatorname{Pic}\left(X_{\Sigma}\right)$ [27, Lemma 4.8], where $\operatorname{Pic}\left(X_{\Sigma}\right)$ is the Picard group of the coarse moduli space $X_{\Sigma}$. On the other hand, we expect that $\mathbb{O}^{\Sigma}$ is the set of classes of orbi-discs in $H_{2}\left(\mathfrak{X}_{\boldsymbol{\Sigma}}, L ; \mathbb{Q}\right) \cong \mathbb{Q}^{R(\boldsymbol{\Sigma})}$ with boundaries in a Lagrangian torus fibre $L \subset \mathfrak{X}_{\boldsymbol{\Sigma}}$. The notation $\mathbb{O}$ indicates 'open'.

We introduce the (extended) Mori cones and their open analogues. Let $\Sigma(n)$ denote the set of $n$-dimensional (i.e., maximal) cones of $\Sigma$. For $\sigma \in \Sigma(n)$, we define

$$
\begin{aligned}
& \widetilde{C}_{\boldsymbol{\Sigma}, \sigma}:=\left\{\lambda \in \mathbb{R}^{S}: \beta(\lambda) \in \sigma, \lambda_{b} \geq 0 \text { for } b \notin R(\boldsymbol{\Sigma}) \cap \sigma\right\}, \\
& C_{\boldsymbol{\Sigma}, \sigma}:=\mathbb{L}_{\mathbb{R}} \cap \widetilde{C}_{\boldsymbol{\Sigma}, \sigma}=\left\{\lambda \in \mathbb{L}_{\mathbb{R}}: \lambda_{b} \geq 0 \text { for } b \notin R(\boldsymbol{\Sigma}) \cap \sigma\right\},
\end{aligned}
$$

where $\lambda_{b}$ with $b \in S$ denotes the $b$ th component of $\lambda \in \mathbb{R}^{S}$. We define the extended Mori cone $\widehat{\mathrm{NE}}\left(\mathfrak{X}_{\boldsymbol{\Sigma}}\right)$ and its open analogue (cone of "open" curves) $\widehat{\mathrm{OE}}\left(\mathfrak{X}_{\boldsymbol{\Sigma}}\right)$ by

$$
\widehat{\mathrm{OE}}\left(\mathfrak{X}_{\boldsymbol{\Sigma}}\right):=\sum_{\sigma \in \Sigma(n)} \widetilde{C}_{\boldsymbol{\Sigma}, \sigma}, \quad \widehat{\mathrm{NE}}\left(\mathfrak{X}_{\boldsymbol{\Sigma}}\right):=\sum_{\sigma \in \Sigma(n)} C_{\boldsymbol{\Sigma}, \sigma}=\widehat{\mathrm{OE}}\left(\mathfrak{X}_{\boldsymbol{\Sigma}}\right) \cap \mathbb{L}_{\mathbb{R}} .
$$

The unextended versions are given by

$$
\mathrm{OE}\left(\mathfrak{X}_{\boldsymbol{\Sigma}}\right):=\widehat{\mathrm{OE}}\left(\mathfrak{X}_{\boldsymbol{\Sigma}}\right) \cap \mathbb{R}^{R(\boldsymbol{\Sigma})}, \quad \mathrm{NE}\left(\mathfrak{X}_{\boldsymbol{\Sigma}}\right):=\widehat{\mathrm{NE}}\left(\mathfrak{X}_{\boldsymbol{\Sigma}}\right) \cap \mathbb{L}_{\mathbb{R}}
$$

The cone $\mathrm{NE}\left(\mathfrak{X}_{\boldsymbol{\Sigma}}\right)$ in $\mathbb{L}_{\mathbb{R}}^{\boldsymbol{\Sigma}} \cong H_{2}\left(\mathfrak{X}_{\boldsymbol{\Sigma}}, \mathbb{R}\right)$ is the usual Mori cone, that is, the cone spanned by effective curves in $\mathfrak{X}_{\boldsymbol{\Sigma}}$. The corresponding monoids are given as follows

$$
\begin{aligned}
& \mathbb{O}(\boldsymbol{\Sigma})_{+}:=\left\{(\lambda, v) \in \mathbb{O}(\boldsymbol{\Sigma}): \lambda \in \widehat{\mathrm{OE}}\left(\mathfrak{X}_{\boldsymbol{\Sigma}}\right)\right\}, \\
& \boldsymbol{\Lambda}(\boldsymbol{\Sigma})_{+}:=\boldsymbol{\Lambda}(\boldsymbol{\Sigma}) \cap \widehat{\mathrm{NE}}\left(\mathfrak{X}_{\boldsymbol{\Sigma}}\right) .
\end{aligned}
$$

Similarly, the unextended versions are given by

$$
\begin{aligned}
& \mathbb{O}_{+}^{\boldsymbol{\Sigma}}:=\mathbb{O}(\boldsymbol{\Sigma})_{+} \cap\left(\mathbb{Q}^{R(\boldsymbol{\Sigma})} \oplus \mathbf{N}\right)=\left\{(\lambda, v) \in \mathbb{O}^{\boldsymbol{\Sigma}}: \lambda \in \mathrm{OE}\left(\mathfrak{X}_{\boldsymbol{\Sigma}}\right)\right\}, \\
& \boldsymbol{\Lambda}_{+}^{\boldsymbol{\Sigma}}:=\boldsymbol{\Lambda}(\boldsymbol{\Sigma})_{+} \cap \mathbb{Q}^{R(\boldsymbol{\Sigma})}=\boldsymbol{\Lambda}^{\boldsymbol{\Sigma}} \cap \mathrm{NE}\left(\mathfrak{X}_{\boldsymbol{\Sigma}}\right) .
\end{aligned}
$$


These cones and monoids are compatible with the decompositions in (3.13) (cf. [62, Lemma 3.2]). It is easy to check that

$$
\begin{array}{ll}
\widehat{\mathrm{OE}}\left(\mathfrak{X}_{\boldsymbol{\Sigma}}\right)=\mathrm{OE}\left(\mathfrak{X}_{\boldsymbol{\Sigma}}\right)+\sum_{b \in G(\boldsymbol{\Sigma})} \mathbb{R}_{\geq 0} \delta_{b}^{\boldsymbol{\Sigma}}, & \mathbb{O}(\boldsymbol{\Sigma})_{+}=\mathbb{O}_{+}^{\boldsymbol{\Sigma}}+\sum_{b \in G(\boldsymbol{\Sigma})} \mathbb{Z}_{\geq 0} \delta_{b}^{\boldsymbol{\Sigma}}, \\
\widehat{\mathrm{NE}}\left(\mathfrak{X}_{\boldsymbol{\Sigma}}\right)=\mathrm{NE}\left(\mathfrak{X}_{\boldsymbol{\Sigma}}\right)+\sum_{b \in G(\boldsymbol{\Sigma})} \mathbb{R}_{\geq 0} \delta_{b}^{\boldsymbol{\Sigma}}, & \boldsymbol{\Lambda}(\boldsymbol{\Sigma})_{+}=\boldsymbol{\Lambda}_{+}^{\boldsymbol{\Sigma}}+\sum_{b \in G(\boldsymbol{\Sigma})} \mathbb{Z}_{\geq 0} \delta_{b}^{\boldsymbol{\Sigma}} .
\end{array}
$$

The following lemma follows immediately from [27, Lemma 2.7].

Lemma 3.12. Consider a natural map $\mathbb{O}(\boldsymbol{\Sigma})_{+} \rightarrow \mathbf{N} \cap \Pi$ given by the second projection. The fibre of this map at $v \in \mathbf{N} \cap \Pi$ equals $\left(\Psi^{\boldsymbol{\Sigma}}(\bar{v}), v\right)+\boldsymbol{\Lambda}(\boldsymbol{\Sigma})_{+}$.

We introduce a pairing between $\operatorname{Pic}^{\mathrm{st}}\left(\mathfrak{X}_{\boldsymbol{\Sigma}}\right):=\operatorname{Pic}\left(\mathfrak{X}_{\boldsymbol{\Sigma}}\right) / \operatorname{Pic}\left(X_{\Sigma}\right)$ and the lattices $\mathbb{O}(\boldsymbol{\Sigma}), \boldsymbol{\Lambda}(\boldsymbol{\Sigma})$, where $\operatorname{Pic}\left(X_{\Sigma}\right)$ denotes the Picard group of the coarse moduli space $X_{\Sigma}$ of $\mathfrak{X}_{\Sigma}$. This pairing corresponds to the Galois symmetry for quantum cohomology in Section 2.2. Note that we have

$$
\boldsymbol{\Lambda}(\boldsymbol{\Sigma}) / \mathbb{L} \cong \mathbb{O}(\boldsymbol{\Sigma}) / \mathbb{Z}^{S} \cong \mathbb{O}^{\boldsymbol{\Sigma}} / \mathbb{Z}^{R(\boldsymbol{\Sigma})},
$$

where the first isomorphism follows from the comparison of the extended fan sequence (3.3) and the extended refined fan sequence (3.11); the second isomorphism follows from the fact that $\mathbb{O}(\boldsymbol{\Sigma})=\mathbb{O}^{\boldsymbol{\Sigma}} \oplus \bigoplus_{b \in G(\boldsymbol{\Sigma})} \mathbb{Z}\left(e_{b}, b\right)$. Recall from $(3.5)$ that Pic $(\mathfrak{X}) \cong \mathbb{L}^{\star} / \sum_{b \in G(\boldsymbol{\Sigma})} \mathbb{Z} D_{b}$. Since we have $\lambda_{b}=D_{b} \cdot \lambda \in \mathbb{Z}$ for $b \in G(\boldsymbol{\Sigma})$ and $\lambda \in \boldsymbol{\Lambda}(\boldsymbol{\Sigma})$, the natural pairing $\mathbb{L}^{\star} \times\left(\mathbb{L}_{\mathbb{Q}} / \mathbb{L}\right) \rightarrow \mathbb{Q} / \mathbb{Z}$ induces the pairing

$$
\text { age: } \operatorname{Pic}\left(\mathfrak{X}_{\boldsymbol{\Sigma}}\right) \times(\boldsymbol{\Lambda}(\boldsymbol{\Sigma}) / \mathbb{L}) \rightarrow \mathbb{Q} / \mathbb{Z}
$$

Through the identification $\boldsymbol{\Lambda}(\boldsymbol{\Sigma}) / \mathbb{L} \cong \mathbb{O}^{\boldsymbol{\Sigma}} / \mathbb{Z}^{R(\boldsymbol{\Sigma})}$ above, this pairing coincides with the age pairing $\operatorname{Pic}\left(\mathfrak{X}_{\boldsymbol{\Sigma}}\right) \times\left(\mathbb{O}^{\boldsymbol{\Sigma}} / \mathbb{Z}^{R(\boldsymbol{\Sigma})}\right) \rightarrow \mathbb{Q} / \mathbb{Z}$ introduced in [27, Section 4.3]. It follows from [27, Lemma 4.7] that the age pairing descends to a perfect pairing

$$
\text { age: } \operatorname{Pic}^{\text {st }}\left(\mathfrak{X}_{\boldsymbol{\Sigma}}\right) \times(\boldsymbol{\Lambda}(\boldsymbol{\Sigma}) / \mathbb{L}) \rightarrow \mathbb{Q} / \mathbb{Z} \text {. }
$$

Via (3.17), we obtain the following pairings:

$$
\begin{array}{rlrl}
\operatorname{Pic}^{\text {st }}\left(\mathfrak{X}_{\boldsymbol{\Sigma}}\right) \times \mathbb{O}(\boldsymbol{\Sigma}) \rightarrow \mathbb{C}^{\times}, & (\xi, x) & \mapsto e^{2 \pi \mathbf{i} \text { age }(\xi, x)}, \\
\operatorname{Pic}^{\mathrm{st}}\left(\mathfrak{X}_{\boldsymbol{\Sigma}}\right) \times \boldsymbol{\Lambda}(\boldsymbol{\Sigma}) \rightarrow \mathbb{C}^{\times}, & (\xi, \lambda) \mapsto e^{2 \pi \mathbf{i} \text { age }(\xi, \lambda)} .
\end{array}
$$

Remark $3.13([27$, Lemma 4.7$])$. For a box element $b \in \operatorname{Box}(\boldsymbol{\Sigma})$, age $\left(\xi,\left(\Psi^{\boldsymbol{\Sigma}}(b), b\right)\right)$ is the age of the line bundle $L_{\xi}$ corresponding to $\xi \in \operatorname{Pic}\left(\mathfrak{X}_{\Sigma}\right)$ along the sector corresponding to the box $b$, where $\left(\Psi^{\boldsymbol{\Sigma}}(b), b\right) \in \mathbb{O}^{\boldsymbol{\Sigma}}$.

\subsection{Local charts of the LG model}

We describe the local charts of $\mathcal{Y}$ and $\mathcal{M}$ corresponding to $\boldsymbol{\Sigma} \in \mathfrak{F} \mathfrak{a n}(S)$. By definition, the local chart of $\mathcal{Y}$ corresponding to $\boldsymbol{\Sigma}$ is given by (see [106, Section 4.1]):

$$
\mathcal{Y}_{\boldsymbol{\Sigma}}=\left[\operatorname{Spec}\left(\mathbb{C}\left[\mathrm{CPL}_{+}(\boldsymbol{\Sigma})^{\vee} \cap \mathrm{PL}_{\mathbb{Z}}(\boldsymbol{\Sigma})^{\star}\right]\right) / \mathcal{G}_{\boldsymbol{\Sigma}}\right]
$$

where

- $\mathrm{CPL}_{+}(\boldsymbol{\Sigma})^{\vee} \subset \mathbb{R}^{S}$ is the dual cone of $\mathrm{CPL}_{+}(\boldsymbol{\Sigma}) \subset\left(\mathbb{R}^{S}\right)^{\star}$ (see (3.8)),

- $\mathrm{PL}_{\mathbb{Z}}(\boldsymbol{\Sigma})^{\star}=\operatorname{Hom}\left(\mathrm{PL}_{\mathbb{Z}}(\boldsymbol{\Sigma}), \mathbb{Z}\right) \subset \mathbb{Q}^{S}$ is the dual lattice of $\mathrm{PL}_{\mathbb{Z}}(\boldsymbol{\Sigma})$ (see (3.9)); 
- $\mathcal{G}_{\boldsymbol{\Sigma}}:=\left(\mathbb{Z}^{S}\right)^{\star} / \mathrm{PL}_{\mathbb{Z}}(\boldsymbol{\Sigma})$ is a finite group; it acts on $\mathbb{C}\left[\mathrm{CPL}_{+}(\boldsymbol{\Sigma})^{\vee} \cap \mathrm{PL}_{\mathbb{Z}}(\boldsymbol{\Sigma})^{\star}\right]$ via the natural pairing $\left(\mathbb{Z}^{S}\right)^{\star} \times \mathrm{PL}_{\mathbb{Z}}(\boldsymbol{\Sigma})^{\star} \rightarrow \mathbb{Q} \rightarrow \mathbb{Q} / \mathbb{Z} \subset \mathbb{C}^{\times}$.

The coarse moduli space of $\mathcal{Y}_{\boldsymbol{\Sigma}}$ is $\operatorname{Spec}\left(\mathbb{C}\left[\operatorname{CPL}_{+}(\boldsymbol{\Sigma})^{\vee} \cap \mathbb{Z}^{S}\right]\right)$. Similarly, the local chart of $\mathcal{M}$ corresponding to $\boldsymbol{\Sigma}$ is given by:

$$
\mathcal{M}_{\boldsymbol{\Sigma}}=\left[\operatorname{Spec}\left(\mathbb{C}\left[\operatorname{cpl}(\boldsymbol{\Sigma})^{\vee} \cap \operatorname{pl}_{\mathbb{Z}}(\boldsymbol{\Sigma})^{\star}\right]\right) / \mathcal{G}_{\boldsymbol{\Sigma}}^{\prime}\right],
$$

where

- $\operatorname{cpl}(\boldsymbol{\Sigma})^{\vee} \subset \mathbb{L}_{\mathbb{R}}$ is the dual cone of $\operatorname{cpl}(\boldsymbol{\Sigma})$ (see (3.10)),

- $\mathrm{pl}_{\mathbb{Z}}(\boldsymbol{\Sigma})^{\star} \subset \mathbb{L}_{\mathbb{Q}}$ is the dual lattice of $\mathrm{pl}_{\mathbb{Z}}(\boldsymbol{\Sigma})=\mathrm{PL}_{\mathbb{Z}}(\boldsymbol{\Sigma}) / \mathbf{M}$ and

- $\mathcal{G}_{\boldsymbol{\Sigma}}^{\prime}:=\mathbb{L}^{\star} / \mathrm{pl}_{\mathbb{Z}}(\boldsymbol{\Sigma})$ acts on $\mathbb{C}\left[\operatorname{cpl}(\boldsymbol{\Sigma})^{\vee} \cap \mathrm{pl}_{\mathbb{Z}}(\boldsymbol{\Sigma})^{\star}\right]$ via the natural pairing $\mathbb{L}^{\star} \times \mathrm{pl}_{\mathbb{Z}}(\boldsymbol{\Sigma})^{\star} \rightarrow$ $\mathbb{Q} \rightarrow \mathbb{Q} / \mathbb{Z} \subset \mathbb{C}^{\times}$.

The coarse moduli space of $\mathcal{M}_{\boldsymbol{\Sigma}}$ is $\operatorname{Spec}\left(\mathbb{C}\left[\operatorname{cpl}(\boldsymbol{\Sigma})^{\vee} \cap \mathbb{L}\right]\right)$. Comparing the extended divisor sequence (3.4) with the sequence $0 \rightarrow \mathbf{M} \rightarrow \mathrm{PL}_{\mathbb{Z}}(\boldsymbol{\Sigma}) \rightarrow \mathrm{pl}_{\mathbb{Z}}(\boldsymbol{\Sigma}) \rightarrow 0$, we obtain the exact sequence

$$
0 \longrightarrow \mathcal{G}_{\boldsymbol{\Sigma}} \longrightarrow \mathcal{G}_{\boldsymbol{\Sigma}}^{\prime} \longrightarrow \operatorname{Ext}^{1}(\mathbf{N}, \mathbb{Z})=\mathbf{N}_{\text {tor }} \longrightarrow 0,
$$

where $\mathbf{N}_{\text {tor }}=\operatorname{Hom}\left(\mathbf{N}_{\text {tor }}, \mathbb{C}^{\times}\right)$denotes the Pontrjagin dual of $\mathbf{N}_{\text {tor }}$. We have $\operatorname{Pic}\left(\mathfrak{X}_{\boldsymbol{\Sigma}}\right) \cong$ $\mathbb{L}^{\star} / \sum_{b \in G(\boldsymbol{\Sigma})} \mathbb{Z} D_{b}$ by (3.5), and that the Picard group $\operatorname{Pic}\left(X_{\Sigma}\right)$ of the coarse moduli space is given by $\operatorname{pl}_{\mathbb{Z}}(\boldsymbol{\Sigma}) / \sum_{b \in G(\boldsymbol{\Sigma})} \mathbb{Z} D_{b}$ (see $[34$, Section 4.2$\left.]\right)$. Therefore

$$
\mathcal{G}_{\Sigma}^{\prime} \cong \operatorname{Pic}^{\mathrm{st}}\left(\mathfrak{X}_{\boldsymbol{\Sigma}}\right)\left(=\operatorname{Pic}\left(\mathfrak{X}_{\Sigma}\right) / \operatorname{Pic}\left(X_{\Sigma}\right)\right)
$$

and the above sequence (3.19) identifies $\mathcal{G}_{\boldsymbol{\Sigma}}$ with the subgroup of $\operatorname{Pic}^{\text {st }}\left(\mathfrak{X}_{\boldsymbol{\Sigma}}\right)$ on which the generic stabilizers $\mathbf{N}_{\text {tor }}$ of $\mathfrak{X}_{\boldsymbol{\Sigma}}$ acts trivially.

We give another description of the local chart $\mathcal{Y}_{\boldsymbol{\Sigma}} \rightarrow \mathcal{M}_{\boldsymbol{\Sigma}}$, which shows that the LG model on this chart is the same as the LG model considered in [27, Section 4].

Lemma 3.14 (duality of cones and lattices).

(1) The extended Mori cone $\widehat{\mathrm{NE}}(\boldsymbol{\Sigma})$ is the dual cone of $\operatorname{cpl}_{+}(\boldsymbol{\Sigma})$; similarly $\widehat{\mathrm{OE}}(\boldsymbol{\Sigma})$ is the dual cone of $\mathrm{CPL}_{+}(\boldsymbol{\Sigma})$.

(2) The lattice $\overline{\mathbb{O}(\boldsymbol{\Sigma})}:=\mathbb{O}(\boldsymbol{\Sigma}) / \mathbf{N}_{\text {tor }}$ equals the dual lattice $\mathrm{PL}_{\mathbb{Z}}(\boldsymbol{\Sigma})^{\star}$; similarly $\boldsymbol{\Lambda}(\boldsymbol{\Sigma})=\mathrm{pl}_{\mathbb{Z}}(\boldsymbol{\Sigma})^{\star}$. Here we identify $\overline{\mathbb{O}(\boldsymbol{\Sigma})}$ with the image of the first projection $\mathbb{O}(\boldsymbol{\Sigma}) \rightarrow \mathbb{Q}^{S}$ (recall that $\mathbf{N}_{\text {tor }}$ is the torsion part of $\mathbb{O}(\boldsymbol{\Sigma})$ ).

Proof. First we prove the statement on the dual cones. Observe that the cone $\mathrm{CPL}_{+}(\boldsymbol{\Sigma})$ can be written as the intersection of the following simplicial cones $K_{\sigma}$ for all maximal cones $\sigma \in \Sigma(n)$ :

$$
K_{\sigma}=\left\{c \in\left(\mathbb{R}^{S}\right)^{\star}: \begin{array}{c}
c_{b} \geq 0(\forall b \in R(\boldsymbol{\Sigma}) \cap \sigma), \text { and the linear function } \\
\varphi: \mathbf{N}_{\mathbb{R}} \rightarrow \mathbb{R} \text { defined by } \varphi(\bar{b})=c_{b}, b \in R(\boldsymbol{\Sigma}) \cap \sigma \\
\text { satisfies } \varphi(\bar{b}) \leq c_{b} \text { for all } b \in S .
\end{array}\right\} .
$$

Recall that $\widehat{\mathrm{OE}}\left(\mathfrak{X}_{\boldsymbol{\Sigma}}\right)$ is the sum of the cones $\widetilde{C}_{\boldsymbol{\Sigma}, \sigma}$ defined in (3.14). Therefore, in order to prove $\mathrm{CPL}_{+}(\boldsymbol{\Sigma})^{\vee}=\widehat{\mathrm{OE}}\left(\mathfrak{X}_{\boldsymbol{\Sigma}}\right)$, it suffices to show that $K_{\sigma}^{\vee}=\widetilde{C}_{\boldsymbol{\Sigma}, \sigma}$. For $b \in S$, we write $\bar{b}=$ $\sum_{b^{\prime} \in R(\boldsymbol{\Sigma}) \cap \sigma} A_{b b^{\prime}} \overline{b^{\prime}}$ with $A_{b b^{\prime}} \in \mathbb{Q}$. Then the cone $K_{\sigma}$ is defined by the linear inequalities:

$$
c_{b} \geq \begin{cases}0, & \text { if } b \in R(\boldsymbol{\Sigma}) \cap \sigma, \\ \sum_{b^{\prime} \in R(\boldsymbol{\Sigma}) \cap \sigma} A_{b b^{\prime}} c_{b^{\prime}}, & \text { if } b \notin R(\boldsymbol{\Sigma}) \cap \sigma .\end{cases}
$$


On the other hand, for $\lambda \in \mathbb{R}^{S}$ and $c \in\left(\mathbb{R}^{S}\right)^{\star}$, we have

$$
c \cdot \lambda=\sum_{b \in R(\boldsymbol{\Sigma}) \cap \sigma} c_{b}\left(\lambda_{b}+\sum_{b^{\prime} \notin R(\boldsymbol{\Sigma}) \cap \sigma} A_{b^{\prime} b} \lambda_{b^{\prime}}\right)+\sum_{b^{\prime} \notin R(\boldsymbol{\Sigma}) \cap \sigma}\left(c_{b^{\prime}}-\sum_{b \in R(\boldsymbol{\Sigma}) \cap \sigma} A_{b^{\prime} b} c_{b}\right) \lambda_{b^{\prime}} .
$$

Hence the dual cone $K_{\sigma}^{\vee}$ is defined by the inequalities $\lambda_{b} \geq 0$ for $b \notin R(\boldsymbol{\Sigma}) \cap \sigma$ and $\lambda_{b}+$ $\sum_{b^{\prime} \notin R(\boldsymbol{\Sigma}) \cap \sigma} A_{b^{\prime} b} \lambda_{b^{\prime}} \geq 0$ for $b \in R(\boldsymbol{\Sigma}) \cap \sigma$. The latter inequality is equivalent to $\beta(\lambda) \in \sigma$, and thus $K_{\sigma}^{\vee}=\widetilde{C}_{\boldsymbol{\Sigma}, \sigma}$. Hence $\mathrm{CPL}_{+}(\boldsymbol{\Sigma})^{\vee}=\widehat{\mathrm{OE}}\left(\mathfrak{X}_{\boldsymbol{\Sigma}}\right)$. The equality $\operatorname{cpl}(\boldsymbol{\Sigma})^{\vee}=\widehat{\mathrm{NE}}\left(\mathfrak{X}_{\boldsymbol{\Sigma}}\right)$ follows from this and $D\left(\mathrm{CPL}_{+}(\boldsymbol{\Sigma})\right)=\operatorname{cpl}(\boldsymbol{\Sigma}), \widehat{\mathrm{NE}}\left(\mathfrak{X}_{\boldsymbol{\Sigma}}\right)=\widehat{\mathrm{OE}}\left(\mathfrak{X}_{\boldsymbol{\Sigma}}\right) \cap \mathbb{L}_{\mathbb{R}}$.

Next we study the dual lattices of $\mathrm{PL}_{\mathbb{Z}}(\boldsymbol{\Sigma}), \operatorname{pl}_{\mathbb{Z}}(\boldsymbol{\Sigma})$. For $c \in\left(\mathbb{R}^{S}\right)^{\star}$ and a maximal cone $\sigma \in \Sigma(n)$, let $m_{\sigma}(c) \in \mathbf{M}_{\mathbb{R}}$ denote the unique element satisfying $m_{\sigma}(c) \cdot b=c_{b}$ for all $b \in R(\boldsymbol{\Sigma}) \cap \sigma$. The lattice $\mathrm{PL}_{\mathbb{Z}}(\boldsymbol{\Sigma})$ is the intersection of the following lattices $L_{\sigma}$ for all $\sigma \in \Sigma(n)$ :

$$
L_{\sigma}=\left\{c \in\left(\mathbb{R}^{S}\right)^{\star}: m_{\sigma}(c) \in \mathbf{M}, c_{b} \in \mathbb{Z} \text { for all } b \notin R(\boldsymbol{\Sigma}) \cap \sigma\right\} .
$$

Therefore $\mathrm{PL}_{\mathbb{Z}}(\boldsymbol{\Sigma})^{\star}=\sum_{\sigma \in \Sigma(n)} L_{\sigma}^{\star}$. For $\lambda \in \mathbb{R}^{S}$ and $c \in\left(\mathbb{R}^{S}\right)^{\star}$, equation (3.20) can be rewritten as:

$$
c \cdot \lambda=m_{\sigma}(c) \cdot \beta(\lambda)+\sum_{b^{\prime} \notin R(\boldsymbol{\Sigma}) \cap \sigma}\left(c_{b^{\prime}}-m_{\sigma}(c) \cdot b^{\prime}\right) \lambda_{b^{\prime}} .
$$

Therefore we have

$$
L_{\sigma}^{\star}=\left\{\lambda \in \mathbb{R}^{S}: \beta(\lambda) \in \overline{\mathbf{N}}=\mathbf{N} / \mathbf{N}_{\text {tor }}, \lambda_{b} \in \mathbb{Z} \text { for all } b \notin R(\boldsymbol{\Sigma}) \cap \sigma\right\} .
$$

On the other hand, $\overline{\mathbb{O}(\boldsymbol{\Sigma})}=\sum_{\sigma \in \Sigma(n)} L_{\sigma}^{\star}$ follows easily from the definition. Thus $\mathrm{PL}_{\mathbb{Z}}(\boldsymbol{\Sigma})^{\star}=\overline{\mathbb{O}(\boldsymbol{\Sigma})}$. The equality $\mathrm{pl}_{\mathbb{Z}}(\boldsymbol{\Sigma})^{\star}=\boldsymbol{\Lambda}(\boldsymbol{\Sigma})$ follows from this, the extended refined fan sequence (3.11) and $\mathrm{pl}_{\mathbb{Z}}(\boldsymbol{\Sigma})=\mathrm{PL}_{\mathbb{Z}}(\boldsymbol{\Sigma}) / \mathbf{M}$

Proposition 3.15. The local chart $\mathcal{Y}_{\boldsymbol{\Sigma}} \rightarrow \mathcal{M}_{\Sigma}$ has the following presentation:

$$
\begin{aligned}
& \mathcal{Y}_{\boldsymbol{\Sigma}} \cong\left[\operatorname{Spec}\left(\mathbb{C}\left[\mathbb{O}(\boldsymbol{\Sigma})_{+}\right]\right) / \operatorname{Pic}^{\mathrm{st}}\left(\mathfrak{X}_{\boldsymbol{\Sigma}}\right)\right], \\
& \mathcal{M}_{\boldsymbol{\Sigma}} \cong\left[\operatorname{Spec}\left(\mathbb{C}\left[\boldsymbol{\Lambda}(\boldsymbol{\Sigma})_{+}\right]\right) / \operatorname{Pic}^{\mathrm{st}}\left(\mathfrak{X}_{\boldsymbol{\Sigma}}\right)\right],
\end{aligned}
$$

where $\operatorname{Pic}^{\text {st }}\left(\mathfrak{X}_{\boldsymbol{\Sigma}}\right)=\operatorname{Pic}\left(\mathfrak{X}_{\boldsymbol{\Sigma}}\right) / \operatorname{Pic}\left(X_{\Sigma}\right)$ acts on $\mathbb{C}\left[\mathbb{O}(\boldsymbol{\Sigma})_{+}\right]$and $\mathbb{C}\left[\boldsymbol{\Lambda}(\boldsymbol{\Sigma})_{+}\right]$via (3.18).

Proof. By the previous Lemma 3.14, we have $\mathbb{O}(\boldsymbol{\Sigma})_{+} / \mathbf{N}_{\text {tor }}=\mathrm{CPL}_{+}(\boldsymbol{\Sigma})^{\vee} \cap \mathrm{PL}_{\mathbb{Z}}(\boldsymbol{\Sigma})^{\star}$ and $\boldsymbol{\Lambda}(\boldsymbol{\Sigma})_{+}=\operatorname{cpl}(\boldsymbol{\Sigma})^{\vee} \cap \operatorname{pl}_{\mathbb{Z}}(\boldsymbol{\Sigma})^{\star}$. Therefore we have the natural maps

$$
\begin{aligned}
& \operatorname{Spec} \mathbb{C}\left[\operatorname{CPL}_{+}(\boldsymbol{\Sigma})^{\vee} \cap \mathrm{PL}_{\mathbb{Z}}(\boldsymbol{\Sigma})^{\star}\right] \hookrightarrow \operatorname{Spec} \mathbb{C}\left[\mathbb{O}(\boldsymbol{\Sigma})_{+}\right], \\
& \operatorname{Spec} \mathbb{C}\left[\operatorname{cpl}(\boldsymbol{\Sigma})^{\vee} \cap \operatorname{pl}_{\mathbb{Z}}(\boldsymbol{\Sigma})^{\star}\right] \cong \operatorname{Spec} \mathbb{C}\left[\boldsymbol{\Lambda}(\boldsymbol{\Sigma})_{+}\right] .
\end{aligned}
$$

The first map is the inclusion of a connected component. We can see that the $\mathcal{G}_{\boldsymbol{\Sigma}}^{\prime}=\mathbb{L}^{\star} / \mathrm{pl}_{\mathbb{Z}}(\boldsymbol{\Sigma}) \cong$ $\operatorname{Pic}^{\text {st }}\left(\mathfrak{X}_{\boldsymbol{\Sigma}}\right)$ action on $\mathbb{C}\left[\operatorname{cpl}(\boldsymbol{\Sigma})^{\vee} \cap \operatorname{pl}_{\mathbb{Z}}(\boldsymbol{\Sigma})^{\star}\right]$ and the $\operatorname{Pic}^{\mathrm{st}}\left(\mathfrak{X}_{\boldsymbol{\Sigma}}\right)$ action on $\mathbb{C}\left[\boldsymbol{\Lambda}(\boldsymbol{\Sigma})_{+}\right]$are the same (both are induced by the pairing $\mathbb{L}^{\star} \times \mathbb{L}_{\mathbb{Q}} \rightarrow \mathbb{Q}$ ). Also the $\operatorname{Pic}^{\mathrm{st}}\left(\mathfrak{X}_{\boldsymbol{\Sigma}}\right)$ action on $\operatorname{Spec} \mathbb{C}\left[\mathbb{O}(\boldsymbol{\Sigma})_{+}\right]$ induces a permutation of its connected components, and the subgroup preserving the component Spec $\mathbb{C}\left[\mathrm{CPL}_{+}(\boldsymbol{\Sigma})^{\vee} \cap \mathrm{PL}_{\mathbb{Z}}(\boldsymbol{\Sigma})^{\star}\right]$ is identified with $\mathcal{G}_{\boldsymbol{\Sigma}}$ (via the sequence (3.19)). The proposition follows. 
It follows from the above proposition that the local chart $\mathcal{Y}_{\Sigma} \rightarrow \mathcal{M}_{\Sigma}$ is a quotient (by $\left.\operatorname{Pic}^{\text {st }}(\mathfrak{X})\right)$ of the LG model considered in [27, Section 4]. Indeed, the decompositions (3.16) induce the isomorphisms

$$
\begin{aligned}
& \operatorname{Spec} \mathbb{C}\left[\mathbb{O}(\boldsymbol{\Sigma})_{+}\right] \cong \operatorname{Spec} \mathbb{C}\left[\mathbb{O}_{+}^{\boldsymbol{\Sigma}}\right] \times \mathbb{C}^{G(\boldsymbol{\Sigma})}, \\
& \operatorname{Spec} \mathbb{C}\left[\boldsymbol{\Lambda}(\boldsymbol{\Sigma})_{+}\right] \cong \operatorname{Spec} \mathbb{C}\left[\boldsymbol{\Lambda}_{+}^{\boldsymbol{\Sigma}}\right] \times \mathbb{C}^{G(\boldsymbol{\Sigma})}
\end{aligned}
$$

and the structure map $\mathcal{Y}_{\boldsymbol{\Sigma}} \rightarrow \mathcal{M}_{\boldsymbol{\Sigma}}$ is induced by the natural map $\operatorname{Spec} \mathbb{C}\left[\mathbb{O}_{+}^{\boldsymbol{\Sigma}}\right] \rightarrow \operatorname{Spec} \mathbb{C}\left[\boldsymbol{\Lambda}_{+}^{\boldsymbol{\Sigma}}\right]$. In [27], the LG model was first introduced on $\operatorname{Spec} \mathbb{C}\left[\mathbb{O}_{+}^{\boldsymbol{\Sigma}}\right] \rightarrow \operatorname{Spec} \mathbb{C}\left[\boldsymbol{\Lambda}_{+}^{\boldsymbol{\Sigma}}\right]$ and then deformed over the parameter space $\mathbb{C}^{G(\boldsymbol{\Sigma})}$. The total deformation family there is identified with the uniformizing chart of the LG model $\left(\operatorname{pr}: \mathcal{Y}_{\boldsymbol{\Sigma}} \rightarrow \mathcal{M}_{\boldsymbol{\Sigma}}, F\right)$ in the present paper. See also the expression (3.22) in explicit co-ordinates and Remark 3.16 below.

\subsection{Co-ordinate system on the local chart}

Using the presentation in Proposition 3.15, we introduce a convenient co-ordinate system on the local chart $\mathcal{Y}_{\boldsymbol{\Sigma}} \rightarrow \mathcal{M}_{\boldsymbol{\Sigma}}$. For $(\lambda, v) \in \mathbb{O}(\boldsymbol{\Sigma})$, we write $u^{(\lambda, v)}$ for the corresponding element in $\mathbb{C}[\mathbb{O}(\boldsymbol{\Sigma})]$. We set

$$
u_{b}:=u^{\left(e_{b}, b\right)} \in \mathbb{C}\left[\mathbb{O}(\boldsymbol{\Sigma})_{+}\right], \quad q^{\lambda}:=u^{(\lambda, 0)} \in \mathbb{C}[\boldsymbol{\Lambda}(\boldsymbol{\Sigma})]
$$

for $b \in S$ and $\lambda \in \boldsymbol{\Lambda}(\boldsymbol{\Sigma})$. When $\lambda$ lies in $\boldsymbol{\Lambda}^{\boldsymbol{\Sigma}} \subset \boldsymbol{\Lambda}(\boldsymbol{\Sigma})$, we also write $\mathrm{q}^{\lambda}$ for $q^{\lambda}$. We choose a splitting $\varsigma: \mathbf{N} \rightarrow \mathbb{O}^{\boldsymbol{\Sigma}}$ of the refined fan sequence (3.12) of the form $\varsigma(v)=(\bar{\varsigma}(v), v)$, where $\bar{\varsigma}: \mathbf{N} \rightarrow \mathbb{Q}^{R(\boldsymbol{\Sigma})}$ defines a splitting of the fan sequence (3.1) over $\mathbb{Q}$. For $v \in \mathbf{N}$ and $b \in S$, we define

$$
\begin{aligned}
& x^{v}:=u^{\varsigma(v)} \in \mathbb{C}[\mathbb{O}(\boldsymbol{\Sigma})], \\
& t_{b}:=q^{\delta_{b}^{\boldsymbol{\Sigma}}}=q^{e_{b}-\Psi^{\boldsymbol{\Sigma}}(b)}=u^{\left(e_{b}-\Psi^{\boldsymbol{\Sigma}}(b), 0\right)} \in \mathbb{C}\left[\boldsymbol{\Lambda}(\boldsymbol{\Sigma})_{+}\right] .
\end{aligned}
$$

Note that $x^{v}$ does not necessarily belong to $\mathbb{C}\left[\mathbb{O}(\boldsymbol{\Sigma})_{+}\right]$and that $t_{b}=1$ for $b \in R(\boldsymbol{\Sigma})$. Then we have for $b \in S$,

$$
u_{b}=t_{b} \mathrm{q}^{\lambda(b)} x^{b},
$$

where $\lambda(b):=\Psi^{\boldsymbol{\Sigma}}(b)-\bar{\varsigma}(b) \in \boldsymbol{\Lambda}^{\boldsymbol{\Sigma}}$. Note that $\mathrm{q}^{\lambda(b)} x^{b}=u^{\left(\Psi^{\boldsymbol{\Sigma}}(b), b\right)} \in \mathbb{C}\left[\mathbb{O}_{+}^{\boldsymbol{\Sigma}}\right]$. We can regard $q=(\mathrm{q}, t)$ as co-ordinates on the base $\mathcal{M}_{\boldsymbol{\Sigma}}$ and $x$ as co-ordinates on fibres of $\mathcal{Y}_{\boldsymbol{\Sigma}} \rightarrow \mathcal{M}_{\boldsymbol{\Sigma}}$. The LG potential

$$
F=\sum_{b \in S} u_{b}=\sum_{b \in R(\boldsymbol{\Sigma})} \mathrm{q}^{\lambda(b)} x^{b}+\sum_{b \in G(\boldsymbol{\Sigma})} t_{b} \mathrm{q}^{\lambda(b)} x^{b}
$$

can then be viewed as a family of Laurent polynomials in $x$ with the fixed set $S$ of exponents. Furthermore, we use the following co-ordinate expressions when necessary.

- Choosing an isomorphism $\mathbf{N} \cong \mathbb{Z}^{n} \times \mathbf{N}_{\text {tor }}$, we write $x^{b}=x_{1}^{b_{1}} \cdots x_{n}^{b_{n}} x^{\zeta}$ when $b \in \mathbf{N}$ corresponds to $\left(b_{1}, \ldots, b_{n}, \zeta\right) \in \mathbb{Z}^{n} \times \mathbf{N}_{\text {tor }} ; x_{1}, \ldots, x_{n}$ can be viewed as co-ordinates along fibres of $\mathcal{Y} \rightarrow \mathcal{M}$ and $x^{\zeta}$ are roots of unity labelling connected components of the fibre.

- Choosing a $\mathbb{Z}$-basis $\lambda_{1}, \ldots, \lambda_{r}$ of $\boldsymbol{\Lambda}^{\boldsymbol{\Sigma}}$, we write $\mathrm{q}_{i}=\mathrm{q}^{\lambda_{i}} \in \mathbb{C}\left[\boldsymbol{\Lambda}^{\boldsymbol{\Sigma}}\right]$; then $\mathrm{q}_{1}, \ldots, \mathrm{q}_{r}$ and $t_{b}$, $b \in G(\boldsymbol{\Sigma})$ together form a co-ordinate system $q=(\mathrm{q}, t)$ on the base.

Remark 3.16. We compare the notation of [27,65] with the present one. In these papers, the LG potential was given in the form

$$
F(x ; y)=\sum_{b \in S} y_{b} w_{b}=\sum_{b \in S} y_{b} Q^{\lambda(b)} x^{b},
$$

where $\left\{y_{b}\right\}_{b \in S}$ are deformation parameters. In the present paper, we $\operatorname{set}^{7} y_{b}=1$ for all $b \in R(\boldsymbol{\Sigma})$.

\footnotetext{
${ }^{7}$ In $[27,65]$, we also wrote $\left\{y_{1}, \ldots, y_{m}\right\}$ for $\left\{y_{b}\right\}_{b \in R(\boldsymbol{\Sigma})}$ with $m=|R(\boldsymbol{\Sigma})|$.
} 
The variables $Q$ and the other variables $y_{b}, b \in G(\boldsymbol{\Sigma})$ correspond to our $\mathrm{q}_{1}, \ldots, \mathrm{q}_{r}$ and $t_{b}$ with $b \in G(\boldsymbol{\Sigma})$, and $w_{b}$ corresponds to our $u^{\left(\Psi^{\boldsymbol{\Sigma}}(b), b\right)}=\mathrm{q}^{\lambda(b)} x^{b}$. (Note that $w_{b}$ does not correspond to $u_{b}$ in the present paper.)

Remark 3.17 ([27, Section 4.1]). By Lemma 3.12, we have

$$
\mathbb{C}\left[\mathbb{O}(\boldsymbol{\Sigma})_{+}\right]=\bigoplus_{v \in \mathbf{N} \cap \Pi} \mathbb{C}\left[\boldsymbol{\Lambda}(\boldsymbol{\Sigma})_{+}\right] u^{\left(\Psi^{\boldsymbol{\Sigma}}(\bar{v}), v\right)}
$$

In particular the family pr: $\mathcal{Y} \rightarrow \mathcal{M}$ is flat. The fact that $\mathbb{C}\left[\mathbb{O}(\boldsymbol{\Sigma})_{+}\right]$is a free $\mathbb{C}\left[\boldsymbol{\Lambda}(\boldsymbol{\Sigma})_{+}\right]$-module played an important role in establishing a mirror isomorphism in [27].

\subsection{Examples}

We give examples of partially compactified LG models for surface singularities.

\subsection{1 $A_{1}$-singularity resolution}

We take $\mathbf{N}=\mathbb{Z}^{2}$ and $S=\{(-1,1),(1,1),(0,1)\}$. There are two stacky fans $\boldsymbol{\Sigma}_{1}, \boldsymbol{\Sigma}_{2}$ adapted to $S$ as shown in Fig. 1. The stacky fan $\boldsymbol{\Sigma}_{1}$ consists of one maximal cone and gives rise to the toric stack $\mathfrak{X}_{1}=\left[\mathbb{C}^{2} / \mu_{2}\right]$ (the $A_{1}$ singularity) and the fan $\boldsymbol{\Sigma}_{2}$ consists of two maximal cones and gives rise to the crepant resolution $\mathfrak{X}_{2}=\mathcal{O}_{\mathbb{P}^{1}}(-2)$ of $\mathbb{C}^{2} / \mu_{2}$.

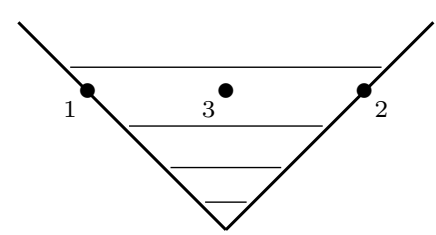

$\Sigma_{1}$

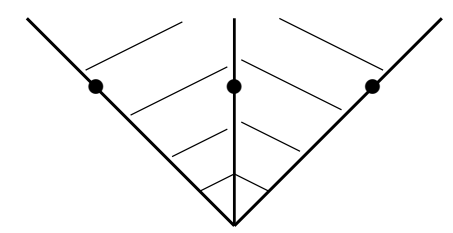

$\Sigma_{2}$

Figure 1. The surface $A_{1}$-singularity (left) and its crepant resolution (right).

The extended fan sequence is

$$
0 \longrightarrow \mathbb{L}=\mathbb{Z} \stackrel{\left(\begin{array}{c}
-1 \\
-1 \\
2
\end{array}\right)}{\longrightarrow} \mathbb{Z}^{3} \stackrel{\left(\begin{array}{ccc}
-1 & 1 & 0 \\
1 & 1 & 1
\end{array}\right)}{\longrightarrow} \mathbf{N}=\mathbb{Z}^{2} \longrightarrow 0
$$

and the extended divisor sequence is its dual

$$
0 \longrightarrow \mathbf{M} \longrightarrow\left(\mathbb{Z}^{3}\right)^{\star} \stackrel{D=(-1,-1,2)}{\longrightarrow} \mathbb{L}^{\star}=\mathbb{Z} \longrightarrow 0 .
$$

The fan $\widetilde{\Xi}$ on the vector space $\left(\mathbb{Z}^{3}\right)^{\star} \otimes \mathbb{R}$ consists of the two maximal cones:

$$
\begin{aligned}
& \mathrm{CPL}_{+}\left(\boldsymbol{\Sigma}_{1}\right)=\left\{\left(c_{1}, c_{2}, c_{3}\right) \in\left(\mathbb{R}_{\geq 0}\right)^{3}: 2 c_{3} \geq c_{1}+c_{2}\right\}, \\
& \mathrm{CPL}_{+}\left(\boldsymbol{\Sigma}_{2}\right)=\left\{\left(c_{1}, c_{2}, c_{3}\right) \in\left(\mathbb{R}_{\geq 0}\right)^{3}: 2 c_{3} \leq c_{1}+c_{2}\right\}
\end{aligned}
$$

and the integral structure $\widetilde{7}$ is given by

$$
\widetilde{\boldsymbol{\tau}}=\left\{\left(c_{1}, c_{2}, c_{3}\right) \in\left(\mathbb{Z}_{\geq 0}\right)^{3}: \min \left(c_{3}, \frac{c_{1}+c_{2}}{2}\right) \in \mathbb{Z}\right\} .
$$

The map $D:\left(\mathbb{Z}^{3}\right)^{\star} \rightarrow \mathbb{L}^{\star}$ induces the secondary fan $\Xi$ on $\mathbb{L}_{\mathbb{R}}^{\star} \cong \mathbb{R}$. The fan $\Xi$ consists of the two maximal cones $\operatorname{cpl}\left(\boldsymbol{\Sigma}_{1}\right)=\mathbb{R}_{\geq 0}, \operatorname{cpl}\left(\boldsymbol{\Sigma}_{2}\right)=\mathbb{R}_{\leq 0}$ and the integral structure is given by 

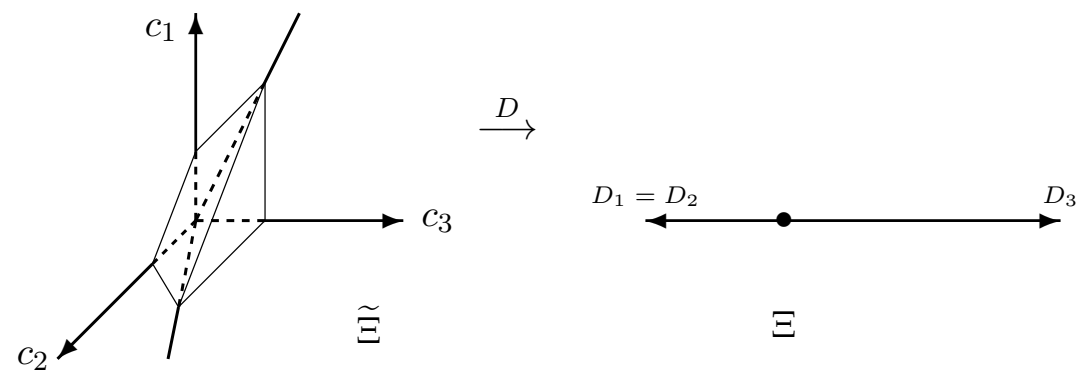

Figure 2. The fans $\widetilde{\Xi}, \Xi$ and the map $D$ between them.

$\boldsymbol{T}=2 \mathbb{Z}_{\geq 0} \cup \mathbb{Z}_{\leq 0}$. See Fig. 2. The extended refined fan sequence (see (3.11)) associated to $\boldsymbol{\Sigma}_{1}$ is given by

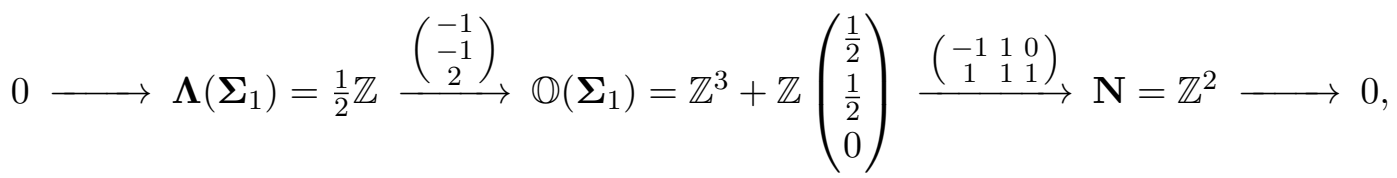

which is a refinement of (3.23). The monoids ${ }^{8}$ corresponding to the (open) Mori cone are given by $\boldsymbol{\Lambda}\left(\boldsymbol{\Sigma}_{1}\right)_{+}=\frac{1}{2} \mathbb{Z}_{\geq 0}$ and $\mathbb{O}\left(\boldsymbol{\Sigma}_{1}\right)_{+}=\mathbb{Z}_{\geq 0}\left\langle e_{1}, e_{2}, \frac{1}{2}\left(e_{1}+e_{2}\right), \delta_{3}\right\rangle$ with $\delta_{3}=\left(-\frac{1}{2},-\frac{1}{2}, 1\right)$. By Proposition 3.15, the chart $\mathcal{Y}_{\boldsymbol{\Sigma}_{1}} \rightarrow \mathcal{M}_{\boldsymbol{\Sigma}_{1}}$ is given by

$$
\begin{gathered}
\mathcal{Y}_{\boldsymbol{\Sigma}_{1}}=\left[\left\{\left(u_{1}, u_{2}, v, t\right): u_{1} u_{2}=v^{2}\right\} / \mu_{2}\right]-F=u_{1}+u_{2}+t v \\
\downarrow_{t}
\end{gathered}
$$

where $u_{1}, u_{2}, v, t$ correspond to $e_{1}, e_{2}, \frac{1}{2}\left(e_{1}+e_{2}\right), \delta_{3} \in \mathbb{O}_{+}\left(\boldsymbol{\Sigma}_{1}\right)$ and $\operatorname{Pic}^{\text {st }}\left(\mathfrak{X}_{\boldsymbol{\Sigma}_{1}}\right)=\mathbb{O}\left(\boldsymbol{\Sigma}_{1}\right) / \mathbb{Z}^{3} \cong \mu_{2}$ acts on these variables by $\left(u_{1}, u_{2}, v, t\right) \mapsto\left(u_{1}, u_{2},-v,-t\right)$. On the other hand, the extended refined fan sequence for $\boldsymbol{\Sigma}_{2}$ is the same as the extended fan sequence (3.23) and one has $\boldsymbol{\Lambda}\left(\boldsymbol{\Sigma}_{2}\right)_{+}=\mathbb{Z}_{\leq 0}$ and $\mathbb{O}\left(\boldsymbol{\Sigma}_{2}\right)_{+}=\mathbb{Z}_{\geq 0}\left\langle e_{1}, e_{2}, e_{3},(1,1,-2)\right\rangle$. Therefore, the chart $\mathcal{Y}_{\boldsymbol{\Sigma}_{2}} \rightarrow \mathcal{M}_{\boldsymbol{\Sigma}_{2}}$ is given by

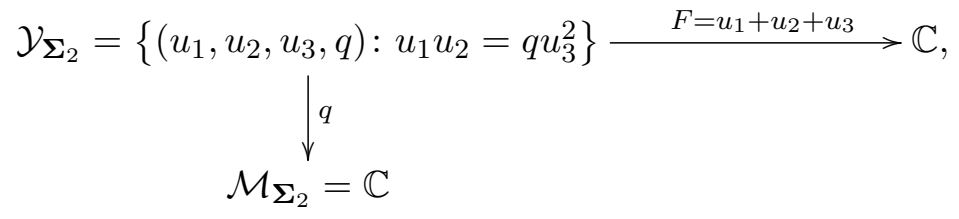

where $u_{1}, u_{2}, u_{3}, q$ correspond respectively to $e_{1}, e_{2}, e_{3},(1,1,-2)$. The global LG model $(\mathcal{Y} \rightarrow \mathcal{M}, F)$ is given by gluing these charts by $u_{3}=v t, q=t^{-2}$. The base space is given by $\mathcal{M}=\mathbb{P}(1,2)$.

\subsubsection{Blowup of $\mathbb{C}^{2}$}

We take $\mathbf{N}=\mathbb{Z}^{2}$ and $S=\{(1,0),(0,1),(1,1)\}$. The possible fan structures $\boldsymbol{\Sigma}_{1}, \boldsymbol{\Sigma}_{2}$ are shown in Fig. 3. The fan $\boldsymbol{\Sigma}_{1}$ corresponds to $\mathbb{C}^{2}$ and $\boldsymbol{\Sigma}_{2}$ corresponds to the blowup $\mathrm{Bl}_{0}\left(\mathbb{C}^{2}\right)$ of $\mathbb{C}^{2}$ at the origin.

\footnotetext{
${ }^{8}$ Note that $\mathbb{O}(\boldsymbol{\Sigma})_{+}=\mathbb{O}(\boldsymbol{\Sigma}) \cap \mathrm{CPL}_{+}(\boldsymbol{\Sigma})^{\vee}$ and $\boldsymbol{\Lambda}(\boldsymbol{\Sigma})_{+}=\boldsymbol{\Lambda}(\boldsymbol{\Sigma}) \cap \operatorname{cpl}(\boldsymbol{\Sigma})^{\vee}$.
} 

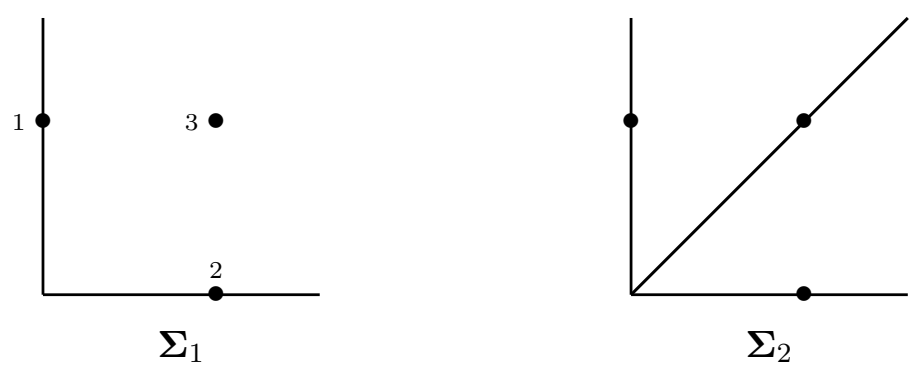

Figure 3. $\mathbb{C}^{2}$ and its blowup at the origin.

In this case, the LG model has no orbifold singularities. The chart $\mathcal{Y}_{\boldsymbol{\Sigma}_{1}} \rightarrow \mathcal{M}_{\boldsymbol{\Sigma}_{1}}$ is given by

$$
\begin{gathered}
\mathcal{Y}_{\boldsymbol{\Sigma}_{1}}=\left\{\left(u_{1}, u_{2}, u_{3}, t\right): t u_{1} u_{2}=u_{3}\right\} \quad F=u_{1}+u_{2}+u_{3} \\
\downarrow_{\boldsymbol{\Sigma}_{1}}=\mathbb{C}
\end{gathered}
$$

The chart $\mathcal{Y}_{\boldsymbol{\Sigma}_{2}} \rightarrow \mathcal{M}_{\boldsymbol{\Sigma}_{2}}$ is given by

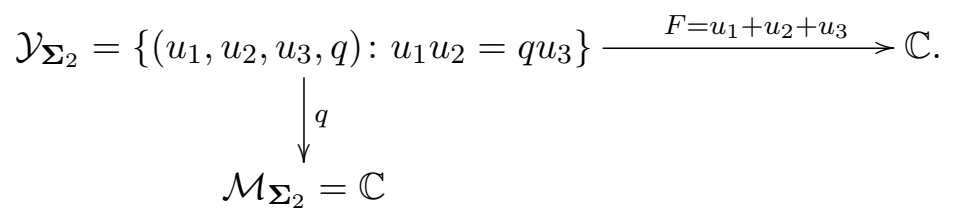

The two charts are glued by $t=q^{-1}$.

\subsubsection{Cyclic quotient singularity}

We take $\mathbf{N}=\mathbb{Z}^{2}$ and $S=\{(0,1),(d,-1),(1,0)\}$ with $d \geq 3$. The case $d=1$ was considered in Section 3.6.2 (blowup of $\mathbb{C}^{2}$ ) and the case $d=2$ was considered in Section 3.6.1 ( $A_{1}$-singularity). As before, there are two fan structures $\boldsymbol{\Sigma}_{1}, \boldsymbol{\Sigma}_{2}$ (see Fig. 4 ). The fan $\boldsymbol{\Sigma}_{1}$ corresponds to $\left[\mathbb{C}^{2} / \mu_{d}\right]$ (of type $\frac{1}{d}(1,1)$ ) and $\boldsymbol{\Sigma}_{2}$ corresponds to its minimal resolution (the total space of $\mathcal{O}(-d)$ over $\mathbb{P}^{1}$ ).
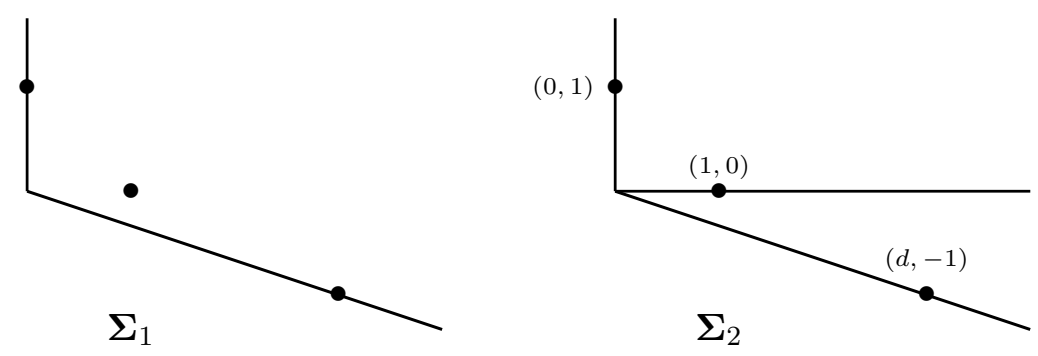

Figure 4. Cyclic quotient singularity and its resolution.

The chart $\mathcal{Y}_{\boldsymbol{\Sigma}_{1}} \rightarrow \mathcal{M}_{\boldsymbol{\Sigma}_{1}}$ is given by

$$
\begin{gathered}
\mathcal{Y}_{\Sigma_{1}}=\left[\left\{\left(u_{1}, u_{2}, v, t\right): u_{1} u_{2}=v^{d}\right\} / \mu_{d}\right]-F=u_{1}+u_{2}+t v \\
\downarrow_{t}
\end{gathered}
$$


where $\operatorname{Pic}^{\text {st }}\left(\mathfrak{X}_{\Sigma_{1}}\right) \cong \mu_{d}$ acts by $\left(u_{1}, u_{2}, v, t\right) \mapsto\left(u_{1}, u_{2}, \zeta v, \zeta^{-1} t\right)$. The chart $\mathcal{Y}_{\boldsymbol{\Sigma}_{2}} \rightarrow \mathcal{M}_{\boldsymbol{\Sigma}_{2}}$ is given by

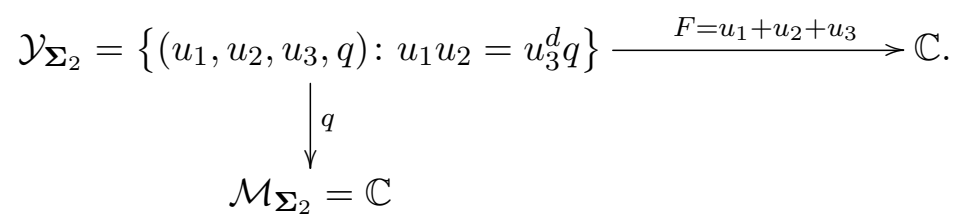

The two charts are glued by $u_{3}=t v$ and $q=t^{-d}$. After gluing, we get $\mathcal{M}_{\boldsymbol{\Sigma}_{1}} \cup \mathcal{M}_{\boldsymbol{\Sigma}_{2}}=\mathbb{P}(1, d)$. The pictures of the fans $\Xi, \widetilde{\Xi}$ are similar to Fig. 2 .

\section{Mirror symmetry}

In this section, we review mirror symmetry for smooth toric DM stacks proved by CoatesCorti-Iritani-Tseng [26, 27] and discuss its analytification. We construct various versions (algebraic, completed, analytified) of Brieskorn modules associated with the Landau-Ginzburg model around the limit point $0_{\boldsymbol{\Sigma}}$ and compare them with the quantum cohomology D-module of the toric stack $\mathfrak{X}_{\boldsymbol{\Sigma}}$. We fix the data $(\mathbf{N}, \Pi, S)$ from Section 3.1 .

\subsection{Brieskorn module}

Let $(\operatorname{pr}: \mathcal{Y} \rightarrow \mathcal{M}, F)$ be the partially compactified LG model associated with the data $(\mathrm{N}, \Pi, S)$ (see Definition 3.6). We adapt the construction of the equivariant Brieskorn module in [27, 65] to our context.

Note that the toric stacks $\mathcal{Y}, \mathcal{M}$ have natural log structures defined by their toric boundaries (see, e.g., [54, Chapter 3]). With respect to these $\log$ structures, the family $\mathcal{Y} \rightarrow \mathcal{M}$ is $\log$ smooth. The sheaves of logarithmic one-forms and logarithmic vector fields on $\mathcal{Y}$ are globally free and given respectively by

$$
\Omega_{\mathcal{Y}}^{1}=\bigoplus_{b \in S} \mathcal{O}_{\mathcal{Y}} \frac{d u_{b}}{u_{b}}, \quad \Theta_{\mathcal{Y}}=\bigoplus_{b \in S} \mathcal{O}_{\mathcal{Y}} u_{b} \frac{\partial}{\partial u_{b}} .
$$

Let $x_{1}, \ldots, x_{n}$ be the co-ordinates on fibres of $\mathcal{Y} \rightarrow \mathcal{M}$ given by the choice of an isomorphism $\mathbf{N} \cong \mathbb{Z}^{n} \times \mathbf{N}_{\text {tor }}$ (see Section 3.5). Then the sheaf of relative logarithmic $k$-forms ${ }^{9}$ are

$$
\Omega_{\mathcal{Y} / \mathcal{M}}^{k}=\bigoplus_{i_{1}<\cdots<i_{k}} \mathcal{O}_{\mathcal{Y}} \frac{d x_{i_{1}}}{x_{i_{1}}} \wedge \cdots \wedge \frac{d x_{i_{k}}}{x_{i_{k}}} .
$$

The sheaf $\Theta_{\mathcal{Y} / \mathcal{M}}$ of relative logarithmic vector fields is generated by $x_{1} \frac{\partial}{\partial x_{1}}, \ldots, x_{n} \frac{\partial}{\partial x_{n}}$. Let $\left\{\chi_{1}, \ldots, \chi_{n}\right\}$ denote the basis of $\mathbf{M}$ dual to the chosen isomorphism $\overline{\mathbf{N}} \cong \mathbb{Z}^{n}$; then the relative vector field $x_{i} \frac{\partial}{\partial x_{i}}$ acts on functions (on the chart $\mathcal{Y}_{\boldsymbol{\Sigma}}$ ) as

$$
x_{i} \frac{\partial}{\partial x_{i}} \cdot u^{(\lambda, b)}=\left(\chi_{i} \cdot b\right) u^{(\lambda, b)} \quad \text { for }(\lambda, b) \in \mathbb{O}(\boldsymbol{\Sigma})_{+} .
$$

For $\xi \in \mathbb{L}_{\mathbb{C}}^{\star}, \xi q \frac{\partial}{\partial q}$ denotes a vector field on $\mathcal{M}$ such that

$$
\xi q \frac{\partial}{\partial q} \cdot q^{\lambda}=(\xi \cdot \lambda) q^{\lambda} \quad \text { for } \lambda \in \boldsymbol{\Lambda}(\boldsymbol{\Sigma})_{+} .
$$

\footnotetext{
${ }^{9}$ The relative $k$-forms $\frac{d x_{i_{1}}}{x_{i_{1}}} \wedge \cdots \wedge \frac{d x_{i_{k}}}{x_{i_{k}}}$ are independent of the choice of a splitting $\varsigma$ in Section 3.5, although the co-ordinates $x_{i}$ themselves depend on $\varsigma$.
} 
We define a generator $\omega$ of $\Omega_{\mathcal{Y} / \mathcal{M}}^{n}$ by

$$
\omega:=\frac{1}{\left|\mathbf{N}_{\text {tor }}\right|} \frac{d x_{1}}{x_{1}} \wedge \cdots \wedge \frac{d x_{n}}{x_{n}}
$$

This is normalized so that the integral over the maximal compact subgroup $\operatorname{Hom}\left(\mathbf{N}, S^{1}\right)$ of $\operatorname{Hom}\left(\mathbf{N}, \mathbb{C}^{\times}\right)$equals $(2 \pi \mathbf{i})^{n}$. Informally speaking, the non-equivariant Brieskorn module below is a D-module on $\mathcal{M} \times \mathbb{C}_{z}$ consisting of certain cohomology classes of relative differential forms $f \omega \in \operatorname{pr}_{*} \Omega_{\mathcal{Y} / \mathcal{M}}^{n}$ such that oscillatory integrals

$$
[f \omega] \longmapsto \int_{\Gamma \subset \mathcal{Y}_{q}} e^{F / z} f(x, q, z) \omega
$$

are solutions to the D-module, where $\mathcal{Y}_{q}:=\operatorname{pr}^{-1}(q)$. In the equivariant case, the phase function $F$ should be replaced with $F-\sum_{i=1}^{n} \chi_{i} \log x_{i}$, see Remark 4.4 below.

\section{Definition 4.1.}

(1) The equivariant Brieskorn module $\operatorname{Bri}_{\mathbb{T}}(F)$ is defined to be the $\mathcal{O}_{\mathcal{Y}}[z]$-module $\Omega_{\mathcal{Y} / \mathcal{M}}^{n}[z]=$ $\mathcal{O}_{\mathcal{Y}}[z] \cdot \omega$ equipped with the flat connection $\nabla: \operatorname{Bri}_{\mathbb{T}}(F) \rightarrow z^{-1} \operatorname{Bri}_{\mathbb{T}}(F) \otimes_{\mathcal{O}_{\mathcal{Y}}} \Omega_{\mathcal{Y}}^{1}$ given by

$$
\nabla_{V}(f \omega)=\left(V(f)+z^{-1} V(F) f\right) \omega
$$

for $f \in \mathcal{O}_{\mathcal{Y}}[z]$ and $V \in \Theta_{\mathcal{Y}}$. We call $\nabla$ the Gauss-Manin connection. We let the equivariant parameter $\chi_{i} \in \mathbf{M}_{\mathbb{C}} \cong H_{\mathbb{T}}^{2}(\mathrm{pt}, \mathbb{C})$ act on $\operatorname{Bri}_{\mathbb{T}}(F)$ by $z \nabla_{x_{i} \frac{\partial}{\partial x_{i}}}$, i.e.,

$$
\chi_{i} \cdot f \omega:=z \nabla_{x_{i} \frac{\partial}{\partial x_{i}}}(f \omega)=\left(z x_{i} \frac{\partial f}{\partial x_{i}}+\sum_{b \in S}\left(\chi_{i} \cdot b\right) u_{b} f\right) \omega
$$

We shall often regard $\operatorname{Bri}_{\mathbb{T}}(F)$ as a sheaf of modules over $\mathcal{M}$ by pushing it forward by pr: $\mathcal{Y} \rightarrow \mathcal{M}$; by abuse of notation we use the same symbol to denote the pushed-forward sheaf. The action of $\chi_{i}$ commutes with the $\mathcal{O}_{\mathcal{M}}[z]$-module structure and thus $\operatorname{Bri}_{\mathbb{T}}(F)$ has the structure of an $\mathcal{O}_{\mathcal{M}} \otimes R_{\mathbb{T}}[z]$-module with $R_{\mathbb{T}}=H_{\mathbb{T}}^{*}(\mathrm{pt}, \mathbb{C}) \cong \operatorname{Sym}\left(\mathbf{M}_{\mathbb{C}}\right)$. The grading operator $\operatorname{Gr} \in \operatorname{End}_{\mathbb{C}}\left(\operatorname{Bri}_{\mathbb{T}}(F)\right)$ is defined by

$$
\operatorname{Gr}(f \omega)=\left(z \frac{\partial f}{\partial z}+\sum_{b \in S} u_{b} \frac{\partial f}{\partial u_{b}}\right) \omega
$$

for $f \in \mathcal{O}_{\mathcal{Y}}[z]$.

(2) The (non-equivariant) Brieskorn module $\operatorname{Bri}(F)$ is defined to be the non-equivariant limit $\operatorname{Bri}_{\mathbb{T}}(F) / \mathbf{M}_{\mathbb{C}} \cdot \operatorname{Bri}_{\mathbb{T}}(F)$ of the equivariant Brieskorn module. This has the structure of an $\mathcal{O}_{\mathcal{M}}[z]$-module. The flat connection and the grading operator on $\operatorname{Bri}_{\mathbb{T}}(F)$ descend to a flat connection $\nabla: \operatorname{Bri}(F) \rightarrow z^{-1} \operatorname{Bri}(F) \otimes \Omega_{\mathcal{M}}^{1}$, also called the Gauss-Manin connection, and an operator $\operatorname{Gr} \in \operatorname{End}_{\mathbb{C}}(\operatorname{Bri}(F))$.

By definition, $\operatorname{Bri}_{\mathbb{T}}(F)$ is isomorphic to the rank-one free module $\mathcal{O}_{\mathcal{Y}}[z]$ equipped with the flat connection $\nabla=d+d(F / z) \wedge$. By pushing it forward by pr: $\mathcal{Y} \rightarrow \mathcal{M}$ and forgetting the action of the fibre co-ordinates $x_{1}, \ldots, x_{n}$, we shall regard $\operatorname{Bri}_{\mathbb{T}}(F)$ as an $\mathcal{O}_{\mathcal{M}} \otimes R_{\mathbb{T}}[z]$-module; 
then $\operatorname{Bri}_{\mathbb{T}}(F)$ is not of rank one as such. ${ }^{10}$ We shall also regard $\operatorname{Bri}_{\mathbb{T}}(F)$ as a flat connection (i.e., D-module) over $\mathcal{M}$. First we regard it as a module over the ring of differential operators

$$
\mathscr{D}=\mathcal{O}_{\mathcal{M}}[z]\left\langle z u_{b} \frac{\partial}{\partial u_{b}}: b \in S\right\rangle,
$$

where $z u_{b} \frac{\partial}{\partial u_{b}}$ act by $z \nabla_{u_{b} \frac{\partial}{\partial u_{b}}}$. Note that $\mathscr{D}$ contains $\mathcal{O}_{\mathcal{M}} \otimes R_{\mathbb{T}}[z]$ as its centre via the map $\mathbf{M}_{\mathbb{C}} \ni$ $\chi_{i} \mapsto z x_{i} \frac{\partial}{\partial x_{i}}$. By choosing a splitting $\left(\mathbb{C}^{S}\right)^{\star} \cong \mathbf{M}_{\mathbb{C}} \oplus \mathbb{L}_{\mathbb{C}}^{\star}$ of the extended divisor sequence (3.4) tensored with $\mathbb{C}$, we can lift a vector field $\xi q \frac{\partial}{\partial q}$ on $\mathcal{M}$ given by $\xi \in \mathbb{L}_{\mathbb{C}}^{\star}$ to a vector field $\hat{\xi} u \frac{\partial}{\partial u}=$ $\sum_{b \in S} \hat{\xi}_{b} u_{b} \frac{\partial}{\partial u_{b}}$ on $\mathcal{Y}$, where $\hat{\xi}=\left(\hat{\xi}_{b}\right)_{b \in S} \in\left(\mathbb{C}^{S}\right)^{\star}$ denotes the lift of $\xi$ under the splitting. By this splitting, we can regard $\operatorname{Bri}_{\mathbb{T}}(F)$ as a module over

$$
\mathscr{D} \cong \mathcal{O}_{\mathcal{M}} \otimes R_{\mathbb{T}}[z]\left\langle z \xi q \frac{\partial}{\partial q}: \xi \in \mathbb{L}_{\mathbb{C}}^{\star}\right\rangle
$$

A different choice of splittings shifts the action of $z \xi q \frac{\partial}{\partial q} \in \Theta_{\mathcal{M}}$ by an element of $\mathbf{M}_{\mathbb{C}}$. When the choice of a splitting is understood, we write $z \nabla_{\xi q} \frac{\partial}{\partial q}$ for $z \nabla_{\hat{\xi} u \frac{\partial}{\partial u}}$. The grading operator Gr satisfies $\left[\mathrm{Gr}, z \nabla_{\xi q \frac{\partial}{\partial q}}\right]=z \nabla_{\xi q \frac{\partial}{\partial q}}$ and

$$
\operatorname{Gr}(f(q, \chi, z) \Omega)=\left(\left(\mathcal{E}+z \frac{\partial}{\partial z}\right) f(q, \chi, z)\right) \Omega+f(q, \chi, z) \operatorname{Gr}(\Omega)
$$

for any $\Omega \in \operatorname{Bri}_{\mathbb{T}}(F)$ and $f(q, z, \chi) \in \mathcal{O}_{\mathcal{M}} \otimes R_{\mathbb{T}}[z]$, where $\mathcal{E}$ is the Euler vector field defined by

$$
\mathcal{E}=\left(\sum_{b \in S} D_{b}\right) q \frac{\partial}{\partial q}+\sum_{i=1}^{n} \chi_{i} \frac{\partial}{\partial \chi_{i}} .
$$

Recall here that $D_{b} \in \mathbb{L}^{\star}$ is the image of $e_{i}^{\star} \in\left(\mathbb{Z}^{S}\right)^{\star}$ under the map $D$ in (3.4) and $\left\{\chi_{1}, \ldots, \chi_{n}\right\}$ denotes a basis of $H_{\mathbb{T}}^{2}(\mathrm{pt})$ so that $R_{\mathbb{T}}=\mathbb{C}\left[\chi_{1}, \ldots, \chi_{n}\right]$.

Remark 4.2. The Brieskorn modules here were called Gauss-Manin systems in our previous papers $[27,65]$. We changed the name because the Gauss-Manin system usually refers to the localization of the Brieskorn module by $z$; the Brieskorn modules were also called Brieskorn lattices in [37, 38, 93, 98].

Remark 4.3. The action of $x_{1}, \ldots, x_{n}$ forgotten in the above process corresponds to the Seidel representation (or shift operator) on quantum cohomology. This defines the structure of a difference module with respect to the equivariant parameters $\chi_{i}$, i.e., the action of $x_{i}$ shifts $\chi_{j}$ as $\chi_{j} \mapsto \chi_{j}-\delta_{i, j} z$ (note that we have the commutation relation $\left[\chi_{i}, x_{j}\right]=z \delta_{i, j} x_{j}$ as operators acting on $\left.\operatorname{Bri}_{\mathbb{T}}(F)\right)$. See [65].

Remark 4.4. As done in $[27,65]$, we can define the equivariant and non-equivariant Brieskorn modules in terms of the twisted (logarithmic) de Rham complex. We have

$$
\begin{aligned}
& \operatorname{Bri}_{\mathbb{T}}(F) \cong \operatorname{pr}_{*} H^{n}\left(\Omega_{\mathcal{Y} / \mathcal{M}}^{\bullet}[z]\left[\chi_{1}, \ldots, \chi_{n}\right], z d+d F_{\mathbb{T}} \wedge\right), \\
& \operatorname{Bri}(F) \cong \operatorname{pr}_{*} H^{n}\left(\Omega_{\mathcal{Y} / \mathcal{M}}^{\bullet}[z], z d+d F \wedge\right)
\end{aligned}
$$

with $F_{\mathbb{T}}=F-\sum_{i=1}^{n} \chi_{i} \log x_{i}$, where $H^{n}(-)$ means the cohomology sheaf of a complex of sheaves (not the hypercohomology $R^{n} \mathrm{pr}_{*}$ ). This definition involves the choice of co-ordinates $x_{1}, \ldots, x_{n}$,

\footnotetext{
${ }^{10}$ This is not coherent as an $\mathcal{O}_{\mathcal{M}} \otimes R_{\mathbb{T}}[z]$-module in general; we shall take its completion in the next section so that it has a finite expected rank.
} 
which corresponds to the choice of a splitting as above. To see that the first isomorphism holds, note that the $n$th cohomology is the cokernel of $z d+d F_{\mathbb{T}} \wedge: \Omega_{\mathcal{Y} / \mathcal{M}}^{n-1}[z][\chi] \rightarrow \Omega_{\mathcal{Y} / \mathcal{M}}^{n}[z][\chi]$ and the relations given by $\operatorname{Im}\left(z d+d F_{\mathbb{T}} \wedge\right)$ define the action of $\chi_{i}$ on $\Omega_{\mathcal{Y} / \mathcal{M}}^{n}[z]$. The second isomorphism follows from the first.

Remark 4.5. Using the local co-ordinates $\mathrm{q}_{1}, \ldots, \mathrm{q}_{r}, t_{b}$ with $b \in G(\boldsymbol{\Sigma})$ on $\mathcal{M}$ from Section 3.5 and the expression (3.22) for $F$, the flat connection $\nabla$ of $\operatorname{Bri}(F)$ and $\operatorname{Bri}_{\mathbb{T}}(F)$ can be written as

$$
\begin{array}{ll}
\nabla_{\mathrm{q}_{i} \frac{\partial}{\partial \mathrm{q}_{i}}}(f \omega)=\left(\mathrm{q}_{i} \frac{\partial f}{\partial \mathrm{q}_{i}}+\frac{f}{z} \sum_{b \in S} t_{b} \lambda(b)_{i} \mathrm{q}^{\lambda(b)} x^{b}\right) \omega & (1 \leq i \leq r), \\
\nabla_{\frac{\partial}{\partial t_{b}}}(f \omega)=\left(\frac{\partial f}{\partial t_{b}}+\frac{f}{z} \mathrm{q}^{\lambda(b)} x^{b}\right) \omega & (b \in G(\boldsymbol{\Sigma})),
\end{array}
$$

where $f \in \mathcal{O}_{\mathcal{Y}}[z]$ and $\lambda(b)_{i}$ denotes the $i$ th component of $\lambda(b) \in \boldsymbol{\Lambda}^{\boldsymbol{\Sigma}}$ with respect to the chosen basis of $\boldsymbol{\Lambda}^{\boldsymbol{\Sigma}}$. Since $\mathrm{q}^{\lambda(b)} x^{b}=u^{\left(\Psi^{\boldsymbol{\Sigma}}(b), b\right)} \in \mathbb{C}\left[\mathbb{O}(\boldsymbol{\Sigma})_{+}\right]$, it follows that $\nabla$ has no singularities along the divisor $t_{b}=0$ with $b \in G(\boldsymbol{\Sigma})$. Therefore, a "smaller" log structure (than the one given by toric boundaries) suffices to describe the logarithmic singularities of $\nabla$. Note that in the equivariant case, the choice of co-ordinates $\mathrm{q}_{i}, t_{b}, x_{i}$ determines the splitting of the extended divisor sequence.

Remark 4.6. In the non-equivariant Brieskorn module, the connection $\nabla$ and the grading operator Gr together define the connection $\nabla_{z \frac{\partial}{\partial z}}=\mathrm{Gr}-\nabla_{\mathcal{E}}-\operatorname{dim} \mathfrak{X} / 2$ in the $z$-direction as in the case of non-equivariant quantum D-modules, where $\mathcal{E}=\left(\sum_{b \in S} D_{b}\right) q \frac{\partial}{\partial q}$ denotes the nonequivariant Euler vector field. Cf. (2.11).

\subsection{Completion and mirror isomorphism}

We introduce a completion of the Brieskorn module at the large radius limit point $0_{\boldsymbol{\Sigma}} \in \mathcal{M}_{\boldsymbol{\Sigma}}$ of $\mathfrak{X}_{\boldsymbol{\Sigma}}$ (see Definition 3.7) and recall a statement on mirror symmetry from [27].

Let $\boldsymbol{\Sigma} \in \mathfrak{F a n}(S)$ be a stacky fan adapted to $S$. Let $\operatorname{Bri}_{\mathbb{T}}(F)_{\boldsymbol{\Sigma}}, \operatorname{Bri}(F)_{\boldsymbol{\Sigma}}$ denote the $\operatorname{Pic}^{\text {st }}\left(\mathfrak{X}_{\boldsymbol{\Sigma}}\right)$ equivariant modules corresponding to $\operatorname{Bri}_{\mathbb{T}}(F), \operatorname{Bri}(F)$ on the affine chart $\mathcal{Y}_{\Sigma} \rightarrow \mathcal{M}_{\Sigma}$ (see Proposition 3.15):

$$
\begin{aligned}
& \operatorname{Bri}_{\mathbb{T}}(F)_{\boldsymbol{\Sigma}}:=\mathbb{C}[z]\left[\mathbb{O}(\boldsymbol{\Sigma})_{+}\right] \cdot \omega \\
& \operatorname{Bri}(F)_{\boldsymbol{\Sigma}}:=\operatorname{Bri}_{\mathbb{T}}(F)_{\boldsymbol{\Sigma}} / \mathbf{M}_{\mathbb{C}} \cdot \operatorname{Bri}_{\mathbb{T}}(F)_{\boldsymbol{\Sigma}}
\end{aligned}
$$

Let $\mathfrak{m}_{\boldsymbol{\Sigma}} \subset \mathbb{C}\left[\boldsymbol{\Lambda}(\boldsymbol{\Sigma})_{+}\right]$denote the maximal ideal corresponding to $0_{\boldsymbol{\Sigma}}$, i.e., the ideal generated by $q^{\lambda}$ with $\lambda \in \boldsymbol{\Lambda}(\boldsymbol{\Sigma})_{+} \backslash\{0\}$.

Definition 4.7. The completed (equivariant and non-equivariant) Brieskorn modules at $0_{\boldsymbol{\Sigma}}$ are defined to be

$$
\begin{aligned}
& \operatorname{Bri}_{\mathbb{T}}(F)_{\boldsymbol{\Sigma}}:=\underbrace{\lim }_{k} \operatorname{Bri}_{\mathbb{T}}(F)_{\boldsymbol{\Sigma}} / \mathfrak{m}_{\boldsymbol{\Sigma}}^{k} \operatorname{Bri}_{\mathbb{T}}(F)_{\boldsymbol{\Sigma}}, \\
& \operatorname{Bri}(F)_{\boldsymbol{\Sigma}}:=\underbrace{\lim }_{\leftarrow} \operatorname{Bri}(F)_{\boldsymbol{\Sigma}} / \mathfrak{m}_{\boldsymbol{\Sigma}}^{k} \operatorname{Bri}(F)_{\boldsymbol{\Sigma}} .
\end{aligned}
$$

The completed equivariant Brieskorn module $\operatorname{Bri}_{\mathbb{T}}(F)^{\wedge}$ is a free $R_{\mathbb{T}}[z] \llbracket \boldsymbol{\Lambda}(\boldsymbol{\Sigma})_{+} \rrbracket$-module of rank $\operatorname{dim} H_{\mathrm{CR}}^{*}(\mathfrak{X})$ [27, Theorem 4.26]. 
We write $\mathcal{M}_{\mathbb{T}}=\mathcal{M} \times \operatorname{Spec} R_{\mathbb{T}}=\mathcal{M} \times$ Lie $\mathbb{T}$ and let

$$
\mathcal{M}_{\mathbb{T}, \boldsymbol{\Sigma}}:=\operatorname{Spf} R_{\mathbb{T}} \llbracket \boldsymbol{\Lambda}(\boldsymbol{\Sigma})_{+} \rrbracket
$$

denote the formal neighbourhood of $0_{\Sigma} \times \operatorname{Spec} R_{\mathbb{T}}$ in $\mathcal{M}_{\mathbb{T}}$. We regard $\operatorname{Bri}_{\mathbb{T}}(F)_{\Sigma}$ as a $\operatorname{Pic}^{\text {st }}(\mathfrak{X})$ equivariant module over $\mathcal{M}_{\mathbb{T}, \boldsymbol{\Sigma}}$. We again choose a splitting $\varsigma: \mathbf{N} \rightarrow \mathbb{O}^{\boldsymbol{\Sigma}}$ of the refined fan sequence (3.12); via the decomposition (3.13), $\varsigma$ induces a splitting of the extended refined fan sequence (3.11) and that of the extended divisor sequence (3.4) over $\mathbb{C}$. As explained in the previous section (Section 4.1), this splitting enables us to regard $\operatorname{Bri}_{\mathbb{T}}(F) \widehat{\Sigma}$ as a partial connection over $\mathcal{M}_{\mathbb{T}, \boldsymbol{\Sigma}} \cdot$ (This partial connection was explicitly described in Remark 4.5 by choosing co-ordinates $\mathrm{q}_{i}, t_{b}, x_{i}$ given by $\varsigma$.)

The splitting $\varsigma$ also defines a splitting $H_{\mathbb{T}}^{2}\left(\mathfrak{X}_{\Sigma}\right) \cong H^{2}\left(\mathfrak{X}_{\Sigma}\right) \oplus H_{\mathbb{T}}^{2}(\mathrm{pt})$ via the isomorphisms (3.6). We choose an $R_{\mathbb{T}^{-}}$basis $\left\{\phi_{i}\right\}_{i=0}^{s}$ of $H_{\mathrm{CR}, \mathbb{T}}^{*}\left(\mathfrak{X}_{\boldsymbol{\Sigma}}\right)$ so that the condition (2.4) is satisfied and that the splitting $H_{\mathbb{T}}^{2}\left(\mathfrak{X}_{\boldsymbol{\Sigma}}\right) \cong \bigoplus_{i=1}^{r} \mathbb{C} \phi_{i} \oplus H_{\mathbb{T}}^{2}(\mathrm{pt})$ given by this basis is the same as the splitting induced by $\varsigma$. This basis defines the equivariant Kähler moduli space $\mathcal{M}_{\mathrm{A}, \mathbb{T}}\left(\mathfrak{X}_{\boldsymbol{\Sigma}}\right)$ and the equivariant quantum D-module $\mathrm{QDM}_{\mathbb{T}}(\mathfrak{X})(2.10)$, see Sections 2.2-2.3. Recall that $\mathrm{QDM}_{\mathbb{T}}(\mathfrak{X})$ is an $H^{2}\left(\mathfrak{X}_{\boldsymbol{\Sigma}}, \mathbb{Z}\right) /\left(\boldsymbol{\Lambda}^{\boldsymbol{\Sigma}}\right)^{\star}$ equivariant module over the formal neighbourhood of the origin in $\mathcal{M}_{\mathrm{A}, \mathbb{T}}\left(\mathfrak{X}_{\boldsymbol{\Sigma}}\right)$; we denote this formal neighbourhood by

$$
\mathcal{M}_{\mathrm{A}, \mathbb{T}}\left(\mathfrak{X}_{\boldsymbol{\Sigma}}\right)^{\wedge}:=\operatorname{Spf} R_{\mathbb{T}}\left[\left[\boldsymbol{\Lambda}_{+}^{\boldsymbol{\Sigma}}\right]\right]\left[\left[\tau^{0}, \tau^{r+1}, \ldots, \tau^{s}\right]\right] .
$$

We have $H^{2}\left(\mathfrak{X}_{\boldsymbol{\Sigma}}, \mathbb{Z}\right) /\left(\boldsymbol{\Lambda}^{\boldsymbol{\Sigma}}\right)^{\star} \cong \operatorname{Pic}^{\text {st }}\left(\mathfrak{X}_{\boldsymbol{\Sigma}}\right)$ because $H^{2}\left(\mathfrak{X}_{\boldsymbol{\Sigma}}, \mathbb{Z}\right) \cong \operatorname{Pic}\left(\mathfrak{X}_{\boldsymbol{\Sigma}}\right)$ and $\left(\boldsymbol{\Lambda}^{\boldsymbol{\Sigma}}\right)^{\star} \cong \operatorname{Pic}\left(X_{\Sigma}\right)$ by [27, Lemma 4.8].

Theorem 4.8 ([27, Theorems 4.28 and 6.11]). Let $\mathfrak{X}_{\Sigma}$ be a smooth toric DM stack from Definition 3.3. There exists a $\operatorname{Pic}^{\mathrm{st}}\left(\mathfrak{X}_{\boldsymbol{\Sigma}}\right)$-equivariant map (mirror map) $\operatorname{mir}: \mathcal{M}_{\mathbb{T}, \boldsymbol{\Sigma}} \rightarrow \mathcal{M}_{\mathrm{A}, \mathbb{T}}\left(\mathfrak{X}_{\boldsymbol{\Sigma}}\right)^{\wedge}$ over $R_{\mathbb{T}}$ and a $\mathrm{Pic}^{\mathrm{st}}\left(\mathfrak{X}_{\boldsymbol{\Sigma}}\right)$-equivariant isomorphism (mirror isomorphism)

Mir: $\operatorname{Bri}_{\mathbb{T}}(F) \widehat{\Sigma} \cong \operatorname{mir}^{*} \operatorname{QDM}_{\mathbb{T}}\left(\mathfrak{X}_{\Sigma}\right)$,

such that

(1) Mir identifies the Gauss-Manin connection on $\operatorname{Bri}_{\mathbb{T}}(F) \widehat{\Sigma}$ with the quantum connection on $\operatorname{mir}^{*} \mathrm{QDM}_{\mathbb{T}}\left(\mathfrak{X}_{\boldsymbol{\Sigma}}\right)$;

(2) mir preserves the Euler vector fields $\operatorname{mir}_{*}(\mathcal{E})=\mathcal{E}$ (see (2.7), (4.2)) and Mir intertwines the grading operators $\mathrm{Gr} \circ \mathrm{Mir}=\operatorname{Mir} \circ \mathrm{Gr}$ (see (2.8), (4.1));

(3) Mir identifies the higher residue pairing (see [27, Section 6] and Section 4.3.3) with the pairing $P(2.9)$ induced by the orbifold Poincaré pairing.

Remark 4.9. The mirror map mir and the mirror isomorphism Mir are obtained from those in [27] by the restriction to $y_{1}=\cdots=y_{m}=1$. See also Remark 3.16.

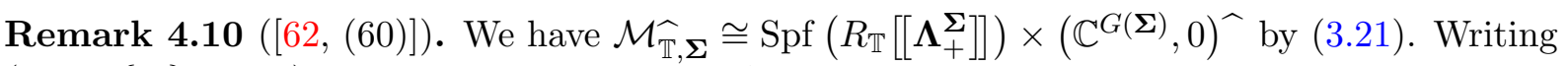
$\left(\mathbf{q}, t=\left\{t_{v}\right\}_{v \in G(\boldsymbol{\Sigma})}\right)$ for the co-ordinates on $\mathcal{M}_{\mathbb{T}, \boldsymbol{\Sigma}}$ as in Section 3.5, we have that the mirror map $(\mathbf{q}, t) \mapsto\left(\hat{\mathrm{q}}, \tau^{\prime}=\tau^{0} \phi_{0}+\sum_{i=r+1}^{s} \tau^{i} \phi_{i}\right)$ has the following asymptotic form

$$
\sum_{i=1}^{r} \phi_{i} \log \hat{\mathbf{q}}_{i}+\tau^{\prime}=\sum_{i=1}^{r} \phi_{i} \log \mathbf{q}_{i}+\sum_{v \in G(\boldsymbol{\Sigma})} t_{v} \mathfrak{D}_{v}+O\left(\mathbf{q}, t^{2}\right),
$$


where

$$
\mathfrak{D}_{v}=\prod_{b \in R(\boldsymbol{\Sigma})} \bar{D}_{b}^{\left\lfloor\Psi_{b}(v)\right\rfloor} \mathbf{1}_{\langle v\rangle} \quad \text { with } \quad\langle v\rangle=v-\sum_{b \in R(\boldsymbol{\Sigma})}\left\lfloor\Psi_{b}(v)\right\rfloor b \in \operatorname{Box}(\boldsymbol{\Sigma}) .
$$

Here we assumed that $\left\{\phi_{i}\right\}_{i=1}^{r} \subset(\mathbb{L} \underset{\mathbb{C}}{\boldsymbol{\Sigma}})^{\star}$ is chosen to be a $\mathbb{Z}$-basis of $\left(\boldsymbol{\Lambda}^{\boldsymbol{\Sigma}}\right)^{\star}$ and that its dual basis defines co-ordinates $\mathrm{q}_{i}, i=1, \ldots, r$ as in Section 3.5; $O\left(\mathrm{q}, t^{2}\right)$ denotes an element of the ideal generated by $\mathrm{q}^{\lambda}\left(\lambda \in \boldsymbol{\Lambda}_{+}^{\boldsymbol{\Sigma}} \backslash\{0\}\right)$ and $t_{b_{1}} t_{b_{2}}\left(b_{1}, b_{2} \in G(\boldsymbol{\Sigma})\right)$.

The above mirror isomorphism shows that $\operatorname{Bri}_{\mathbb{T}}(F)_{\boldsymbol{\Sigma}}$ is a free $R_{\mathbb{T}}[z] \llbracket \boldsymbol{\Lambda}(\boldsymbol{\Sigma})_{+} \rrbracket$-module of rank $\operatorname{dim} H_{\mathrm{CR}}^{*}\left(\mathfrak{X}_{\Sigma}\right)$. This fact shows the following two propositions. The first one proves a nonequivariant version of Theorem 4.8.

Proposition 4.11. We have an isomorphism

$$
\operatorname{Bri}(F) \widehat{\Sigma} \cong \operatorname{Bri}_{\mathbb{T}}(F) \widehat{\Sigma} / \mathbf{M}_{\mathbb{C}} \operatorname{Bri}_{\mathbb{T}}(F) \widehat{\Sigma} .
$$

In particular, the completed Brieskorn module $\operatorname{Bri}(F) \widehat{\Sigma}$ is a free module of rank $\operatorname{dim} H_{\mathrm{CR}}^{*}(\mathfrak{X})$ over $\mathbb{C}[z] \llbracket \boldsymbol{\Lambda}(\boldsymbol{\Sigma})_{+} \rrbracket$ and we have a non-equivariant mirror isomorphism

$$
\left.\operatorname{Mir}\right|_{\chi=0}: \operatorname{Bri}(F)_{\boldsymbol{\Sigma}} \cong \operatorname{mir}^{*} \operatorname{QDM}\left(\mathfrak{X}_{\boldsymbol{\Sigma}}\right),
$$

where mir denotes the non-equivariant limit of the mirror map in Theorem 4.8.

Proof. We apply Lemma 4.13 below to $K=\mathbb{C}\left[\boldsymbol{\Lambda}(\boldsymbol{\Sigma})_{+}\right], I=\mathfrak{m}_{\boldsymbol{\Sigma}}, N=\operatorname{Bri}_{\mathbb{T}}(F)_{\boldsymbol{\Sigma}}^{\oplus n}, M=$ $\operatorname{Bri}_{\mathbb{T}}(F)_{\Sigma}$ and the map $f=\bigoplus_{i=1}^{n} \chi_{i}$. Then we find that $\operatorname{Cok}(f)^{\wedge}=\operatorname{Bri}(F)_{\boldsymbol{\Sigma}}$ is the $\mathfrak{m}_{\boldsymbol{\Sigma}}$-adic completion of $\operatorname{Cok}(\hat{f})=\operatorname{Bri}_{\mathbb{T}}(F) \widehat{\Sigma} / \mathbf{M}_{\mathbb{C}} \operatorname{Bri}_{\mathbb{T}}(F) \widehat{\Sigma}$. On the other hand, $\operatorname{Cok}(\hat{f})$ is a free $\mathbb{C}[z] \llbracket \boldsymbol{\Lambda}(\boldsymbol{\Sigma})_{+} \rrbracket-$ module of finite rank, and in particular $\mathfrak{m}_{\boldsymbol{\Sigma}}$-adically complete. This proves the proposition.

Proposition 4.12. We have

$$
\operatorname{Bri}_{\mathbb{T}}(F)_{\boldsymbol{\Sigma}} / z \operatorname{Bri}_{\mathbb{T}}(F)_{\boldsymbol{\Sigma}} \cong \lim _{k} \mathbb{C}\left[\mathbb{O}(\boldsymbol{\Sigma})_{+}\right] / \mathfrak{m}_{\boldsymbol{\Sigma}}^{k}
$$

Proof. We apply Lemma 4.13 to $K=\mathbb{C}\left[\boldsymbol{\Lambda}(\boldsymbol{\Sigma})_{+}\right], I=\mathfrak{m}_{\boldsymbol{\Sigma}}, N=M=\operatorname{Bri}_{\mathbb{T}}(F)_{\boldsymbol{\Sigma}}$ and the map $f=z$. Then we find that the $\mathfrak{m}_{\boldsymbol{\Sigma}}$-adic completion of $\operatorname{Cok}(f)=\mathbb{C}\left[\mathbb{O}(\boldsymbol{\Sigma})_{+}\right]$is isomorphic to the $\mathfrak{m}_{\boldsymbol{\Sigma}}$-adic completion of $\operatorname{Cok}(\hat{f})=\operatorname{Bri}_{\mathbb{T}}(F)_{\boldsymbol{\Sigma}} / z \operatorname{Bri}_{\mathbb{T}}(F)_{\boldsymbol{\Sigma}}$. Here $\operatorname{Cok}(\hat{f})$ is a free $R_{\mathbb{T}} \llbracket \boldsymbol{\Lambda}(\boldsymbol{\Sigma})_{+} \rrbracket-$ module of finite rank, and in particular $\mathfrak{m}_{\Sigma}$-adically complete. The conclusion follows.

Lemma 4.13. Let $K$ be a ring and $I \subset K$ be a finitely generated ideal. Let $N, M$ be $K$-modules and $f: N \rightarrow M$ be a homomorphism of $K$-modules. Let $\widehat{N}, \widehat{M}$ denote the I-adic completions of $N$ and $M$ respectively and let $\hat{f}: \widehat{N} \rightarrow \widehat{M}$ denote the induced homomorphism. Then the I-adic completion of $\operatorname{Cok}(f)$ is isomorphic to the I-adic completion of $\operatorname{Cok}(\hat{f})$.

Proof. Since $I$ is finitely generated, we have $\widehat{N} / I^{k} \widehat{N} \cong N / I^{k} N$ and $\widehat{M} / I^{k} \widehat{M} \cong M / I^{k} M$ (see [103, Lemma 05GG]). Therefore the exact sequences $N \rightarrow M \rightarrow \operatorname{Cok}(f) \rightarrow 0$ and $\widehat{N} \rightarrow$ $\widehat{M} \rightarrow \operatorname{Cok}(\hat{f}) \rightarrow 0$ imply (by the right-exactness of $\otimes_{K}\left(K / I^{k}\right)$ ) that $\operatorname{Cok}(f) / I^{k} \operatorname{Cok}(f) \cong$ $\operatorname{Cok}(\hat{f}) / I^{k} \operatorname{Cok}(\hat{f})$. The conclusion follows.

Remark 4.14 (cf. Remark 4.4). In [27,65], the completed Brieskorn modules are described as twisted de Rham cohomology. We have

$$
\begin{aligned}
& \operatorname{Bri}_{\mathbb{T}}(F)_{\boldsymbol{\Sigma}} \cong \operatorname{pr}_{*} H^{n}\left(\Omega_{\mathcal{Y} / \mathcal{M}}^{\bullet}[z]^{\wedge}\left[\chi_{1}, \ldots, \chi_{n}\right], z d+d F_{\mathbb{T}} \wedge\right), \\
& \operatorname{Bri}(F)_{\widehat{\Sigma}} \cong \operatorname{pr}_{*} H^{n}\left(\Omega_{\mathcal{Y} / \mathcal{M}}^{\bullet}[z]^{\curlywedge}, z d+d F \wedge\right),
\end{aligned}
$$


where $\widehat{ }$ means the $\mathfrak{m}_{\boldsymbol{\Sigma}}$-adic completion. These isomorphisms follow from the same argument as in Remark 4.4. Note that $\left(\Omega_{\mathcal{Y} / \mathcal{M}}[z]^{\wedge}, z d+d F \wedge\right)$ is the Koszul complex associated with the action of $\chi_{1}, \ldots, \chi_{n}$ on $\operatorname{Bri}_{\mathbb{T}}(F)_{\Sigma}^{\widehat{\Sigma}}=\mathcal{O}_{\mathcal{Y}}[z]^{\wedge} \cdot \omega$. Since we know that $\operatorname{Bri}_{\mathbb{T}}(F)_{\boldsymbol{\Sigma}}$ is a free $R_{\mathbb{T}}[z] \llbracket \boldsymbol{\Lambda}(\boldsymbol{\Sigma})_{+} \rrbracket$-module, $\chi_{1}, \ldots, \chi_{n}$ form a regular sequence for the module $\operatorname{Bri}_{\mathbb{T}}(F) \hat{\Sigma}^{\widehat{\Sigma}}$. Thus we have

$$
H^{i}\left(\Omega_{\mathcal{Y} / \mathcal{M}}[z]^{\wedge}, z d+d F \wedge\right)=0 \quad \text { for } i \neq n .
$$

Therefore, we have $\operatorname{Bri}(F) \widehat{\boldsymbol{\Sigma}} \cong R^{n} \operatorname{pr}_{*}\left(\Omega_{\mathcal{Y} / \mathcal{M}}[z]^{\wedge}, z d+d F \wedge\right)$.

\subsection{Analytification of the completed Brieskorn module}

In this section, we construct an analytification of the completed Brieskorn module. There is a trade-off ${ }^{11}$ between the analyticity along $\mathcal{M}_{\mathbb{T}}=\mathcal{M} \times$ Lie $\mathbb{T}$ and that along the $z$-plane: the analytified Brieskorn module is analytic in the $\mathcal{M}_{\mathbb{T}}$-direction but formal in the variable $z$.

\subsubsection{Analytification of algebras}

First we study the restriction of $\operatorname{Bri}_{\mathbb{T}}(F)$ to $z=0$. By definition, $\operatorname{Bri}_{\mathbb{T}}(F) / z \operatorname{Bri}_{\mathbb{T}}(F)$ is isomorphic to $\mathcal{O}_{\mathcal{Y}}$ and the $R_{\mathbb{T}} \otimes \mathcal{O}_{\mathcal{M}}$-module structure on it is induced by the map

$$
\widetilde{\operatorname{pr}}:=\left(\operatorname{pr}, x_{1} \frac{\partial F}{\partial x_{1}}, \ldots, x_{n} \frac{\partial F}{\partial x_{n}}\right): \mathcal{Y} \rightarrow \mathcal{M}_{\mathbb{T}}=\mathcal{M} \times \operatorname{Lie} \mathbb{T},
$$

where Lie $\mathbb{T}=\operatorname{Spec} R_{\mathbb{T}}$ is identified with $\mathbb{C}^{n}$ via the basis $\chi_{1}, \ldots, \chi_{n}$ chosen in Section 4.1. Let $\tilde{0}=\tilde{0}_{\boldsymbol{\Sigma}} \in \mathcal{Y}_{\boldsymbol{\Sigma}} \subset \mathcal{Y}$ denote the (unique) torus-fixed point on the chart $\mathcal{Y}_{\boldsymbol{\Sigma}}$ such that $\operatorname{pr}\left(\tilde{0}_{\boldsymbol{\Sigma}}\right)=0_{\boldsymbol{\Sigma}}$ : it is defined by $u^{(\lambda, v)}=0$ for all non-torsion elements $(\lambda, v) \in \mathbb{O}(\boldsymbol{\Sigma})_{+}$. On the uniformizing chart Spec $\mathbb{C}\left[\mathbb{O}(\boldsymbol{\Sigma})_{+}\right]$of $\mathcal{Y}_{\boldsymbol{\Sigma}}, \tilde{0}_{\boldsymbol{\Sigma}}$ corresponds to $\left|\mathbf{N}_{\text {tor }}\right|$ many points $\mathbf{N}_{\text {tor }}^{\wedge} \cong \operatorname{Spec} \mathbb{C}\left[\mathbf{N}_{\text {tor }}\right]$ which form a single $\mathrm{Pic}^{\mathrm{st}}\left(\mathfrak{X}_{\boldsymbol{\Sigma}}\right)$-orbit (recall the sequence (3.19)). We mean by $\tilde{0}_{\boldsymbol{\Sigma}}$ either a single point in $\mathcal{Y}_{\boldsymbol{\Sigma}}$ or the corresponding finite subset in $\operatorname{Spec} \mathbb{C}\left[\mathbb{O}(\boldsymbol{\Sigma})_{+}\right]$depending on the context. We have $\widetilde{\operatorname{pr}}\left(\tilde{0}_{\boldsymbol{\Sigma}}\right)=\left(0_{\boldsymbol{\Sigma}}, 0\right)$. In order to avoid the heavy notation, in this Section 4.3 , we will sometimes omit the subscript $\boldsymbol{\Sigma}$ for $0_{\boldsymbol{\Sigma}}$ and $\tilde{0}_{\boldsymbol{\Sigma}}$, writing $0 \in \mathcal{M}_{\boldsymbol{\Sigma}}$ for $0_{\boldsymbol{\Sigma}}$ and $\tilde{0} \in \mathcal{Y}_{\boldsymbol{\Sigma}}$ for $\tilde{0}_{\boldsymbol{\Sigma}}$.

Lemma 4.15. The set-theoretic fibre of $\widetilde{\operatorname{pr}}$ at $(0,0)$ is $\tilde{0}$, i.e., $\tilde{0}=\widetilde{\mathrm{pr}}^{-1}(0,0)$.

Proof. Note that

$$
x_{i} \frac{\partial F}{\partial x_{i}}=\sum_{b \in S}\left(\chi_{i} \cdot b\right) u_{b} \in \sum_{b \in R(\boldsymbol{\Sigma})}\left(\chi_{i} \cdot b\right) u_{b}+\mathfrak{m}_{\boldsymbol{\Sigma}} \mathbb{C}\left[\mathbb{O}(\boldsymbol{\Sigma})_{+}\right],
$$

since $t_{b} \in \mathfrak{m}_{\boldsymbol{\Sigma}}$ for $b \in G(\boldsymbol{\Sigma})$ (see (3.22)). Therefore the scheme theoretic fibre at $(0,0)$ is the spectrum of

$$
\mathbb{C}\left[\mathbb{O}(\boldsymbol{\Sigma})_{+}\right] /\left(\mathfrak{m}_{\boldsymbol{\Sigma}} \mathbb{C}\left[\mathbb{O}(\boldsymbol{\Sigma})_{+}\right]+\left\langle\sum_{b \in R(\boldsymbol{\Sigma})}\left(\chi_{i} \cdot b\right) u_{b}: 1 \leq i \leq n\right\rangle\right) .
$$

By Remark 3.17, we have

$$
\mathbb{C}\left[\mathbb{O}(\boldsymbol{\Sigma})_{+}\right] / \mathfrak{m}_{\boldsymbol{\Sigma}} \mathbb{C}\left[\mathbb{O}(\boldsymbol{\Sigma})_{+}\right] \cong \bigoplus_{v \in \mathbf{N} \cap \Pi} \mathbb{C} w_{v} \quad \text { with } w_{v}:=\left[u^{\left(\Psi^{\boldsymbol{\Sigma}}(\bar{v}), v\right)}\right],
$$

\footnotetext{
${ }^{11}$ By the convergence result from [26] reviewed in Section 4.4, we can make the analytified Brieskorn module fully analytic both in the $\mathcal{M}_{\mathbb{T}}$-direction and in the $z$-direction; the full analytification is given by the analytic quantum D-module. This analytic structure is in general different from that of the original (algebraic) Brieskorn module; the gauge transformation $\left(M_{i}^{j}(q, \chi, z)\right.$ ) (see Part (b) in Section 4.4) connecting the Brieskorn module and the quantum D-module is only a formal power series in $z$. This stems from the fact that the (extended) $I$-function [26] is not necessarily convergent: see [59, Proposition 5.13] for a relevant statement in the non-weak-Fano case.
} 
where $\Psi^{\boldsymbol{\Sigma}}$ is given in Notation 3.10 and the product on the right-hand side is given by

$$
w_{v_{1}} \cdot w_{v_{2}}= \begin{cases}w_{v_{1}+v_{2}}, & \text { if } v_{1}, v_{2} \text { lie in the same cone of } \Sigma, \\ 0, & \text { otherwise. }\end{cases}
$$

It follows that the ring (4.4) is precisely the presentation of the orbifold cohomology ring $H_{\mathrm{CR}}^{*}\left(\mathfrak{X}_{\boldsymbol{\Sigma}}\right)$ due to Borisov-Chen-Smith [14]. Since elements in $H_{\mathrm{CR}}^{>0}\left(\mathfrak{X}_{\boldsymbol{\Sigma}}\right)$ are nilpotent, the set-theoretical fibre at $(0,0)$ equals $\operatorname{Spec}\left(H_{\mathrm{CR}}^{0}\left(\mathfrak{X}_{\boldsymbol{\Sigma}}\right)\right) \cong \operatorname{Spec}\left(\mathbb{C}\left[\mathbf{N}_{\text {tor }}\right]\right)=\tilde{0}$.

We use the following elementary lemma on general topology.

Lemma 4.16. Let $X$ and $Y$ be locally compact Hausdorff spaces and let $f: X \rightarrow Y$ be a continuous map. Let $y_{0} \in Y$ be such that $f^{-1}\left(y_{0}\right)$ is compact. Then

(1) there exist an open neighbourhood $B$ of $f^{-1}\left(y_{0}\right)$ and an open neighbourhood $U$ of $y_{0}$ such that $f(B) \subset U$ and $\left.f\right|_{B}: B \rightarrow U$ is proper;

(2) the family of open sets $\left\{f^{-1}(V) \cap B: V\right.$ is an open neighbourhood of $\left.y_{0}\right\}$ is a fundamental neighbourhood system of $f^{-1}\left(y_{0}\right)$.

Proof. Since $X$ is locally compact, we can find a relatively compact open neighbourhood $C$ of $f^{-1}\left(y_{0}\right)$. Then $\bar{C} \backslash C$ is compact and hence $f(\bar{C} \backslash C)$ is a compact set not containing $y_{0}$. Since $Y$ is Hausdorff, $f(\bar{C} \backslash C)$ is closed; thus there exists an open neighbourhood $U$ of $y_{0}$ such that $U \cap f(\bar{C} \backslash C)=\varnothing$. Then one sees easily that $f^{-1}(U) \cap C=f^{-1}(U) \cap \bar{C}$. For every compact subset $K$ in $U$, we have that $f^{-1}(K) \cap C=f^{-1}(K) \cap \bar{C}$ is compact. This shows that $\left.f\right|_{C \cap f^{-1}(U)}$ is proper. It now suffices to set $B=C \cap f^{-1}(U)$ to conclude Part (1).

To see Part (2), take an open neighbourhood $W \subset B$ of $f^{-1}\left(y_{0}\right)$. Then $B \backslash W$ is closed in $B$. Since proper maps between locally compact Hausdorff spaces are closed, $f(B \backslash W)$ is closed in $U$ and does not contain $y_{0}$. Thus there exists an open neighbourhood $V \subset U$ of $y_{0}$ such that $V \cap f(B \backslash W)=\varnothing$. Then $f^{-1}(V) \cap B \subset W$ and Part (2) follows.

Lemma 4.17. There exist an analytic open neighbourhood $\mathcal{B}$ of $\tilde{0}$ in $\mathcal{Y}_{\boldsymbol{\Sigma}}$ and an analytic open neighbourhood $\mathcal{U}$ of $(0,0)$ in $\mathcal{M}_{\boldsymbol{\Sigma}} \times \operatorname{Lie} \mathbb{T} \subset \mathcal{M}_{\mathbb{T}}$ such that $\widetilde{\operatorname{pr}}(\mathcal{B}) \subset \mathcal{U}$ and that the map $\left.\widetilde{\mathrm{pr}}\right|_{\mathcal{B}}: \mathcal{B} \rightarrow \mathcal{U}$ is proper. Moreover, for any sheaf $\mathcal{F}$ on $\mathcal{B}$ (with respect to the complex-analytic topology), we have $\left(\widetilde{\mathrm{pr}}_{*} \mathcal{F}\right)_{(0,0)}=\mathcal{F}_{\tilde{0}}$, where $\left(\widetilde{\mathrm{pr}}_{*} \mathcal{F}\right)_{(0,0)}$ and $\mathcal{F}_{\tilde{0}}$ denote the stalks respectively at $(0,0)$ and $\tilde{0}$.

Proof. We apply Lemma 4.16 for $f=\widetilde{p r}, X=\mathcal{Y}, Y=\mathcal{M}_{\mathbb{T}}$ and $y_{0}=(0,0)$. Note that Lemma 4.15 gives $f^{-1}\left(y_{0}\right)=\tilde{0}$. The former statement follows from Part (1) of Lemma 4.16 and the latter statement on stalks follows from Part (2).

We will use the notation $\mathcal{O}^{\text {an }}$ to denote the complex-analytic structure sheaf. The following lemma shows that $\widetilde{p r}_{*} \mathcal{O}_{\mathcal{B}}^{\text {an }}$ (which is coherent by Grauert's direct image theorem) gives an analytification of $\operatorname{Bri}_{\mathbb{T}}(F) \widehat{\Sigma} / z \operatorname{Bri}_{\mathbb{T}}(F) \widehat{\Sigma}$.

Lemma 4.18. Let $\mathcal{B} \subset \mathcal{Y}$ and $\mathcal{U} \subset \mathcal{M}_{\mathbb{T}}=\mathcal{M} \times$ Lie $\mathbb{T}$ be as in Lemma 4.17. (They are analytic open neighbourhoods of $\tilde{0}$ and $(0,0)$ respectively. $)$ Let $\left(\widetilde{\operatorname{pr}}_{*} \mathcal{O}_{\mathcal{B}}^{\text {an }}\right)_{(0,0)}$ denote the completion of $\widetilde{\mathrm{pr}}_{*} \mathcal{O}_{\mathcal{B}}^{\text {an }}$ at $(0,0) \in \mathcal{U}$. We have

$$
\left(\widetilde{\operatorname{pr}}_{*} \mathcal{O}_{\mathcal{B}}^{\mathrm{an}}\right)_{(0,0)} \cong \widehat{\mathcal{O}}_{\mathcal{Y}, \tilde{0}} \cong\left(\operatorname{Bri}_{\mathbb{T}}(F)_{\boldsymbol{\Sigma}} / z \operatorname{Bri}_{\mathbb{T}}(F)_{\boldsymbol{\Sigma}}\right) \otimes_{R_{\mathbb{T}} \llbracket \boldsymbol{\Lambda}(\boldsymbol{\Sigma})_{+} \rrbracket} \widehat{\mathcal{O}}_{\mathcal{U},(0,0)}
$$

Here $\widehat{\mathcal{O}}_{\mathcal{U},(0,0)}$ is the completion of $\mathcal{O}_{\mathcal{U}}^{\text {an }}$ at $(0,0)$ and $\widehat{\mathcal{O}}_{\mathcal{Y}, \tilde{0}}$ is the completion of $\mathcal{O}_{\mathcal{Y}}$ at $\tilde{0}$; more explicitly, they are

$$
\widehat{\mathcal{O}}_{\mathcal{U},(0,0)}=\widehat{R_{\mathbb{T}}} \llbracket \Lambda(\boldsymbol{\Sigma})_{+} \rrbracket, \quad \widehat{\mathcal{O}}_{\mathcal{Y}, \tilde{0}}=\mathbb{C} \llbracket \mathbb{O}(\boldsymbol{\Sigma})_{+} \rrbracket,
$$


where $\widehat{R_{\mathbb{T}}}=\mathbb{C} \llbracket \chi_{1}, \ldots, \chi_{n} \rrbracket$ is the completion of $R_{\mathbb{T}}=\mathbb{C}\left[\chi_{1}, \ldots, \chi_{n}\right]$, and for a ring $K, K \llbracket \mathbb{O}(\boldsymbol{\Sigma})_{+} \rrbracket$ denotes the completion of $K\left[\mathbb{O}(\boldsymbol{\Sigma})_{+}\right]$with respect to the ideal generated by $u^{(\lambda, v)}$ with non-torsion $(\lambda, v) \in \mathbb{O}(\boldsymbol{\Sigma})_{+}$.

Proof. We show that the natural maps

$$
\begin{aligned}
& \left(\widetilde{\operatorname{pr}}_{*} \mathcal{O}_{\mathcal{B}}^{\text {an }}\right)_{(0,0)} \rightarrow \widehat{\mathcal{O}}_{\mathcal{Y}, \tilde{0}}, \\
& \left(\operatorname{Bri}_{\mathbb{T}}(F)_{\boldsymbol{\Sigma}} / z \operatorname{Bri}_{\mathbb{T}}(F)_{\boldsymbol{\Sigma}}\right) \otimes_{R_{\mathbb{T}} \llbracket \boldsymbol{\Lambda}(\boldsymbol{\Sigma})_{+} \rrbracket} \widehat{\mathcal{O}}_{\mathcal{U},(0,0)} \rightarrow \widehat{\mathcal{O}}_{\mathcal{Y}, \tilde{0}}
\end{aligned}
$$

are isomorphisms. First we prove that the map (4.6) is an isomorphism. The left-hand side of (4.6) is the $\mathfrak{m}_{(0,0)}^{\text {an }}$-adic completion of $\left(\widetilde{\mathrm{pr}}_{*} \mathcal{O}_{\mathcal{B}}^{\text {an }}\right)_{(0,0)}$, where $\mathfrak{m}_{(0,0)}^{\text {an }} \subset \mathcal{O}_{\mathcal{U},(0,0)}^{\text {an }}$ is the ideal of $(0,0)$. Recall from Lemma 4.15 that the set-theoretic fibre of $\widetilde{\text { pr at }}(0,0)$ is $\tilde{0}$; also from Lemma 4.17 that $\left(\widetilde{\mathrm{pr}}_{*} \mathcal{O}_{\mathcal{B}}^{\mathrm{an}}\right)_{(0,0)}=\mathcal{O}_{\mathcal{B}, \tilde{0}}^{\text {an }}$. Therefore the ideal $\mathfrak{m}_{\tilde{0}}^{\text {an }} \subset \mathcal{O}_{\mathcal{B}, \tilde{0}}^{\text {an }}$ of $\tilde{0}$ is the radical of the ideal generated by $\mathfrak{m}_{(0,0)}^{\text {an }}$, and the $\mathfrak{m}_{(0,0)}^{\text {an }}$-adic topology on $\left(\widetilde{\mathrm{pr}}_{*} \mathcal{O}_{\mathcal{B}}^{\text {an }}\right)_{(0,0)}=\mathcal{O}_{\mathcal{B}, \tilde{0}}^{\text {an }}$ is equivalent to the $\mathfrak{m}_{\tilde{0}}^{\text {an }}$-adic topology on the same space. The completion with respect to the latter topology equals $\widehat{\mathcal{O}}_{\mathcal{B}, \tilde{0}}=\widehat{\mathcal{O}}_{\mathcal{Y}, \tilde{0}}$.

We prove similarly that the map (4.7) is an isomorphism. Recall that $\operatorname{Bri}_{\mathbb{T}}(F)_{\boldsymbol{\Sigma}} / z \operatorname{Bri}_{\mathbb{T}}(F)_{\boldsymbol{\Sigma}}$ is a finite free $R_{\mathbb{T}} \llbracket \boldsymbol{\Lambda}(\boldsymbol{\Sigma})_{+} \rrbracket$-module by Theorem 4.8. Also $\widehat{\mathcal{O}}_{\mathcal{U},(0,0)}$ is the completion of $R_{\mathbb{T}} \llbracket \boldsymbol{\Lambda}(\boldsymbol{\Sigma})_{+} \rrbracket$ with respect to the ideal $\widehat{\mathfrak{m}}_{(0,0)}$ generated by $\chi_{1}, \ldots, \chi_{n}$ and $\mathfrak{m}_{\boldsymbol{\Sigma}}$. Therefore the left-hand side of (4.7) is the $\widehat{\mathfrak{m}}_{(0,0)}$-adic completion of $\operatorname{Bri}_{\mathbb{T}}(F)_{\boldsymbol{\Sigma}} / z \operatorname{Bri}_{\mathbb{T}}(F)_{\boldsymbol{\Sigma}}$ (see, e.g., [103, Lemma 00MA]). Moreover $\operatorname{Bri}_{\mathbb{T}}(F)_{\boldsymbol{\Sigma}} / z \operatorname{Bri}_{\mathbb{T}}(F)_{\boldsymbol{\Sigma}}$ is the $\mathfrak{m}_{\boldsymbol{\Sigma}}$-adic completion of $\mathbb{C}\left[\mathbb{O}(\boldsymbol{\Sigma})_{+}\right]$by Proposition 4.12. Thus $^{12}$ the left-hand side of $(4.7)$ is the $\mathfrak{m}_{(0,0)}$-adic completion of $\mathbb{C}\left[\mathbb{O}(\boldsymbol{\Sigma})_{+}\right]$, where $\mathfrak{m}_{(0,0)}:=$ $\mathfrak{m}_{\boldsymbol{\Sigma}}+\left(\chi_{1}, \ldots, \chi_{n}\right)$ is an ideal of $R_{\mathbb{T}}\left[\boldsymbol{\Lambda}(\boldsymbol{\Sigma})_{+}\right]$. Again Lemma 4.15 implies that the $\mathfrak{m}_{(0,0)}$-adic completion of $\mathbb{C}\left[\mathbb{O}(\boldsymbol{\Sigma})_{+}\right]$equals the $\mathfrak{m}_{\tilde{0}}$-adic completion of it, where $\mathfrak{m}_{\tilde{0}} \subset \mathbb{C}\left[\mathbb{O}(\boldsymbol{\Sigma})_{+}\right]$is the ideal of $\tilde{0}$. This shows that the map (4.7) is an isomorphism. The lemma is proved.

Because $\operatorname{Bri}_{\mathbb{T}}(F)_{\boldsymbol{\Sigma}} / z \operatorname{Bri}_{\mathbb{T}}(F)_{\boldsymbol{\Sigma}}$ is a free $R_{\mathbb{T}} \llbracket \boldsymbol{\Lambda}(\boldsymbol{\Sigma})_{+} \rrbracket$-module of rank dim $H_{\mathrm{CR}}^{*}\left(\mathfrak{X}_{\boldsymbol{\Sigma}}\right)$ (by Theorem 4.8), we have the following corollary.

Corollary 4.19. The modules (4.5) are free $\widehat{\mathcal{O}}_{\mathcal{U},(0,0)}$-modules of rank $\operatorname{dim} H_{\mathrm{CR}}^{*}\left(\mathfrak{X}_{\boldsymbol{\Sigma}}\right)$.

Corollary 4.20. Let $\mathcal{B} \subset \mathcal{Y}$ and $\mathcal{U} \subset \mathcal{M}_{\mathbb{T}}$ be as in Lemma 4.17. By shrinking $\mathcal{B}, \mathcal{U}$ if necessary, we have that $\widetilde{\mathrm{pr}}_{*} \mathcal{O}_{\mathcal{B}}^{\text {an }}$ is a locally free $\mathcal{O}_{\mathcal{U}}^{\text {an }}$-module of rank $\operatorname{dim} H_{\mathrm{CR}}^{*}\left(\mathfrak{X}_{\boldsymbol{\Sigma}}\right)$. In particular, $\widetilde{\mathrm{pr}}: \mathcal{B} \rightarrow \mathcal{U}$ is a finite flat morphism.

Proof. Note that $\widetilde{\mathrm{pr}}_{*} \mathcal{O}_{\mathcal{B}}^{\text {an }}$ is a coherent $\mathcal{O}_{\mathcal{U}}^{\text {an }}$-module by Grauert's Direct Image Theorem and Lemma 4.17. Thus it suffices to show that the stalk $\left(\widetilde{\mathrm{pr}}_{*} \mathcal{O}_{\mathcal{B}}^{\text {an }}\right)_{(0,0)}$ is a free $\mathcal{O}_{\mathcal{U},(0,0)}^{\text {an }}$-module of rank $\operatorname{dim} H_{\mathrm{CR}}^{*}\left(\mathfrak{X}_{\boldsymbol{\Sigma}}\right)$. By Corollary 4.19, we know that the completion $\left(\widetilde{\mathrm{pr}}_{*} \mathcal{O}_{\mathcal{B}}^{\text {an }}\right)_{(0,0)}^{\widehat{0}}$ is a free $\widehat{\mathcal{O}}_{\mathcal{U},(0,0)}$-module of rank $\operatorname{dim} H_{\mathrm{CR}}^{*}\left(\mathfrak{X}_{\boldsymbol{\Sigma}}\right)$. The conclusion follows from the following (probably) well-known fact: for a Noetherian local ring $(A, \mathfrak{m})$ and a finite $A$-module $M, M$ is a free $A$ module of rank $r$ if and only if its $\mathfrak{m}$-adic completion $\widehat{M}=M \otimes_{A} \widehat{A}$ is a free $\widehat{A}$-module of rank $r$. This follows, for instance, by combining the fact that a finite flat module over a local ring is free, [82, Theorem $22.4(1)]$ and $M / \mathfrak{m} M \cong \widehat{M} / \mathfrak{m} \widehat{M}$.

\subsubsection{Analytification of D-modules}

Next we construct an extension of the completed Brieskorn module $\operatorname{Bri}_{\mathbb{T}}(F)_{\widehat{\Sigma}}$ to an analytic neighbourhood of $\left(0_{\Sigma}, 0\right) \in \mathcal{M}_{\mathbb{T}}=\mathcal{M} \times \operatorname{Lie} \mathbb{T}$. Let $\mathcal{U} \subset \mathcal{M}_{\mathbb{T}}$ and $\mathcal{B} \subset \mathcal{Y}$ denote analytic open

\footnotetext{
${ }^{12}$ Here we used the following fact. Let $I \subset J$ be ideals of a $\operatorname{ring} R$, let $M$ be an $R$-module and assume that $I$ is

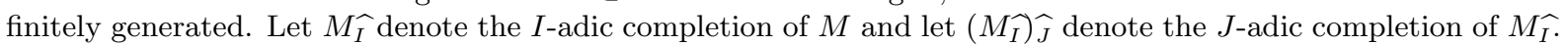
Then $\left(M_{I}\right) \widehat{J} \cong M_{J}$. This follows from $M_{I} / J^{k} M_{I} \cong\left(M_{I} / I^{k} M_{I}\right) / J^{k}\left(M_{I}^{\widehat{I}} / I^{k} M_{I}\right) \cong\left(M / I^{k}\right) / J^{k}\left(M / I^{k}\right) \cong M / J^{k} M$ (where we used [103, Lemma $05 \mathrm{GG}]$ in the middle step).
} 
neighbourhoods of $(0,0)=\left(0_{\Sigma}, 0\right)$ and $\tilde{0}=\tilde{0}_{\Sigma}$ respectively as in Corollary 4.20. Recall that $\widetilde{\mathrm{pr}}_{*} \mathcal{O}_{\mathcal{B}}^{\text {an }}$ is a locally free $\mathcal{O}_{\mathcal{U}}^{\text {an }}$-module by Corollary 4.20 .

Definition 4.21 (cf. Definition 4.1). Let $\boldsymbol{\Sigma} \in \mathfrak{F a n}(S)$ be a stacky fan adapted to $S$.

(1) The analytified equivariant Brieskorn module around the limit point $0=0_{\boldsymbol{\Sigma}}$ is the sheaf over $\mathcal{U}$

$$
\overline{\operatorname{Bri}_{\mathbb{T}}^{\mathrm{an}}}(F)_{\Sigma}:=\widetilde{\operatorname{pr}}_{*}\left(\mathcal{O}_{\mathcal{B}}^{\mathrm{an}} \llbracket z \rrbracket\right) \cdot \omega .
$$

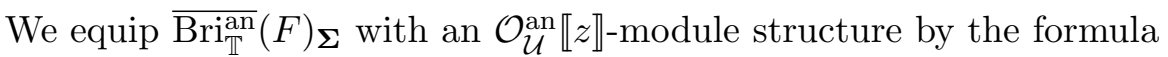

$$
\chi_{i} \cdot(f \omega)=\left(x_{i} \frac{\partial F}{\partial x_{i}} f+z x_{i} \frac{\partial f}{\partial x_{i}}\right) \omega
$$

for $f \in \widetilde{p r}_{*}\left(\mathcal{O}_{\mathcal{B}}^{\text {an }} \llbracket z \rrbracket\right)$, together with the usual multiplication of functions in $q$ and $z$ (where $\chi_{i}$ is a co-ordinate on Lie $\mathbb{T}$ and $q$ is a co-ordinate on $\mathcal{M}$, see Sections 3.5 and 4.1). A flat connection $\nabla=d+d(F / z) \wedge$ on $\mathcal{O}_{\mathcal{B}}^{\text {an }} \llbracket z \rrbracket$ induces, via the choice of a splitting $\left(\mathbb{C}^{S}\right)^{\star} \cong \mathbf{M}_{\mathbb{C}} \oplus$ $\mathbb{L}_{\mathbb{C}}^{\star}$ of the extended divisor sequence (3.4) over $\mathbb{C}$, a partial (logarithmic) flat connection on $\overline{\operatorname{Bri}_{\mathbb{T}}^{a n}}(F)_{\Sigma}$

$$
\nabla: \overline{\operatorname{Bri}_{\mathbb{T}}^{\text {an }}}(F)_{\Sigma} \rightarrow z^{-1} \overline{\operatorname{Bri}_{\mathbb{T}}^{\text {an }}}(F) \otimes_{\mathcal{O}_{\mathcal{U}}^{\text {an }}} \Omega_{\mathcal{U} / \text { Lie } \mathbb{T}}^{1, \text { an }}
$$

in the direction of $\mathcal{M}$, see the discussion after Definition 4.1. A grading operator $\mathrm{Gr} \in$ $\operatorname{End}_{\mathbb{C}}\left(\overline{\operatorname{Bri}_{\mathbb{T}}^{\mathrm{an}}}(F)_{\boldsymbol{\Sigma}}\right)$ is defined as before

$$
\operatorname{Gr}(f \omega)=\left(z \frac{\partial f}{\partial z}+\sum_{b \in S} u_{b} \frac{\partial f}{\partial u_{b}}\right) \omega
$$

(2) The (non-equivariant) analytified Brieskorn module $\overline{\operatorname{Bri}^{\mathrm{an}}}(F)_{\boldsymbol{\Sigma}}$ around the limit point 0 is defined to be the restriction of $\overline{\operatorname{Bri}_{\mathbb{T}}^{\text {an }}}(F)_{\Sigma}$ to $\mathcal{V}=(\mathcal{M} \times\{0\}) \cap \mathcal{U}$. It is equipped with the flat connection $\nabla: \overline{\operatorname{Bri}^{\mathrm{an}}}(F)_{\boldsymbol{\Sigma}} \rightarrow z^{-1} \overline{\operatorname{Bri}^{\mathrm{an}}}(F)_{\boldsymbol{\Sigma}} \otimes \Omega_{\mathcal{V}}^{1, \text { an }}$ (independent of the choice of a splitting) and the grading operator $\operatorname{Gr} \in \operatorname{End}_{\mathbb{C}}\left(\overline{\operatorname{Bri}}^{{ }^{a n}}(F)_{\Sigma}\right)$. The connection $\nabla$ and $\mathrm{Gr}$ together give a flat connection in the $z$-direction as in Remark 4.6.

Remark 4.22. The overline for $\overline{\operatorname{Bri}_{\mathbb{T}}^{\mathrm{an}}}(F)_{\boldsymbol{\Sigma}}, \overline{\mathrm{Bri}^{\mathrm{an}}}(F)_{\boldsymbol{\Sigma}}$ indicates that they are completed in the $z$-adic topology. Note also that these analytified Brieskorn modules depend on the choice of $\boldsymbol{\Sigma} \in \mathfrak{F a n}(S)$.

Remark 4.23. The $\mathcal{O}_{\mathcal{U}}^{\text {an } \llbracket z \rrbracket-m o d u l e ~ s t r u c t u r e ~ o n ~} \overline{\operatorname{Bri}_{\mathbb{T}}^{\text {an }}}(F)_{\Sigma}$ is not a standard one on $\widetilde{\operatorname{pr}}_{*}\left(\mathcal{O}_{\mathcal{B}}^{\text {ann }} \llbracket z \rrbracket\right) \omega$. A more precise definition of the module structure is described as follows: we let a function $h=h\left(q, \chi_{1}, \ldots, \chi_{n}, z\right) \in \mathcal{O}_{\mathcal{U}}^{\text {an }} \llbracket z \rrbracket$ act on a section $f(q, x, z) \omega$ of $\overline{\operatorname{Bri}_{\mathbb{T}}^{\text {an }}}(F)_{\boldsymbol{\Sigma}}$ as

$$
h \cdot(f \omega)=\left[h\left(q, x_{1} \frac{\partial F}{\partial x_{1}}+z x_{1} \frac{\partial}{\partial x_{1}}, \ldots, x_{n} \frac{\partial F}{\partial x_{n}}+z x_{n} \frac{\partial}{\partial x_{n}}, z\right) f(q, x, z)\right] \omega,
$$

where in the right-hand side we expand $h\left(q, x_{1} \frac{\partial F}{\partial x_{1}}+z x_{1} \frac{\partial}{\partial x_{1}}, \ldots, x_{n} \frac{\partial F}{\partial x_{n}}+z x_{n} \frac{\partial}{\partial x_{n}}, z\right)$ in power series of $z x_{i} \frac{\partial}{\partial x_{i}}$ and apply it to $f$. Note that $h\left(q, x_{1} \frac{\partial F}{\partial x_{1}}, \ldots, x_{n} \frac{\partial F}{\partial x_{n}}, z\right)$ is the pull-back of $h\left(q, \chi_{1}, \ldots, \chi_{n}, z\right)$ under $\widetilde{\mathrm{pr}}$ since $x_{i} \frac{\partial F}{\partial x_{i}}=\widetilde{\mathrm{pr}}^{*}\left(\chi_{i}\right)$. The action is well-defined, since we allow any formal power series in $z$.

Proposition 4.24. $\overline{\mathrm{Bri}_{\mathbb{T}}^{\mathrm{an}}}(F)_{\boldsymbol{\Sigma}}$ is a locally free $\mathcal{O}_{\mathcal{U}}^{\mathrm{an}} \llbracket z \rrbracket$-module of rank $\operatorname{dim} H_{\mathrm{CR}}^{*}\left(\mathfrak{X}_{\boldsymbol{\Sigma}}\right)$. In partic-

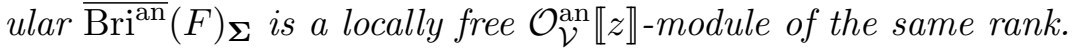


Proof. This follows from Corollary 4.20. Recall that the $\mathcal{O}_{\mathcal{U}}^{\text {an } \llbracket z \rrbracket-m o d u l e ~ s t r u c t u r e ~ o n ~} \overline{\operatorname{Bri}} \overline{\mathbb{T}}(F)_{\Sigma}$ equals the standard one on $\operatorname{pr}_{*}\left(\mathcal{O}_{\mathcal{B}} \llbracket z \rrbracket\right)$ modulo $z$. We show that any local basis $s_{1}, \ldots, s_{N}$ of $\widetilde{\mathrm{pr}}_{*} \mathcal{O}_{\mathcal{B}}^{\text {an }}$ over $\mathcal{O}_{\mathcal{U}}^{\text {an }}$ gives rise to a local basis of $\overline{\operatorname{Bri}_{\mathbb{T}}^{\text {an }}}(F)_{\boldsymbol{\Sigma}}$ over $\mathcal{O}_{\mathcal{U}}^{\text {an }} \llbracket z \rrbracket$. That $s_{1}, \ldots, s_{N}$ generate

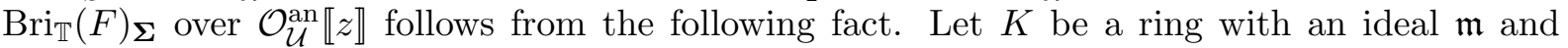
let $M$ be a $K$-module. Suppose that $K$ is $\mathfrak{m}$-adically complete and $M$ is Hausdorff with respect to the $\mathfrak{m}$-adic topology, that is, $\bigcap_{i>0} \mathfrak{m}^{i} M=\{0\}$. If $s_{1}, \ldots, s_{N} \in K$ generate $M / \mathfrak{m} M$ over $K / \mathfrak{m}$, then $s_{1}, \ldots, s_{N}$ generate $M$ over $K$. See for instance [108, Corollary 2, Section 3, Chapter VIII]. We apply this fact for $K=\mathcal{O}_{\mathcal{U}}^{\mathrm{an}} \llbracket z \rrbracket, \mathfrak{m}=z K, M=\overline{\operatorname{Bri}_{\mathbb{T}}^{\mathrm{an}}}(F)_{\boldsymbol{\Sigma}}$. On the other hand, suppose

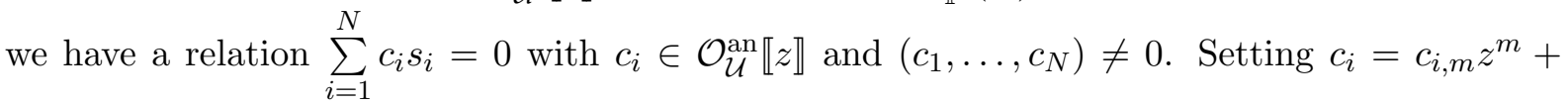
$O\left(z^{m+1}\right)$ for some $m \geq 0$ and $c_{i, m} \in \mathcal{O}_{\mathcal{U}}^{\text {an }}$ with $\left(c_{1, m}, \ldots, c_{N, m}\right) \neq 0$, we obtain a non-trivial relation $\sum_{i=1}^{N} c_{i, m} s_{i}=0$ in $\widetilde{\operatorname{pr}}_{*} \mathcal{O}_{\mathcal{B}}^{\text {an }}$ over $\mathcal{O}_{\mathcal{U}}^{\text {an }}$. This is a contradiction. Thus $s_{1}, \ldots, s_{N}$ are linearly

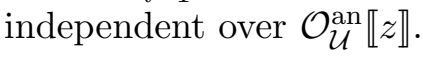

The next proposition shows that $\overline{\operatorname{Bri}_{\mathbb{T}}^{a n}}(F)_{\Sigma}$ is an analytification of $\operatorname{Bri}_{\mathbb{T}}(F)_{\boldsymbol{\Sigma}}$ (and thus justifies the name).

Proposition 4.25 (cf. Lemma 4.18). Let $\left(\overline{\mathrm{Bri}_{\mathbb{T}}^{\text {an }}}(F)_{\boldsymbol{\Sigma}}\right)_{(0,0)}$ denote the $\mathfrak{m}_{(0,0)}^{\text {an }}$-adic completion of $\left(\overline{\operatorname{Bri}_{\mathbb{T}}^{\text {an }}}(F)_{\boldsymbol{\Sigma}}\right)_{(0,0)}$, where $\mathfrak{m}_{(0,0)}^{\text {an }} \subset \mathcal{O}_{\mathcal{U},(0,0)}^{\text {an }}$ is the ideal of $(0,0)$. Then

(1) $\left(\overline{\operatorname{Bri}_{\mathbb{T}}^{\text {an }}}(F)_{\boldsymbol{\Sigma}} \widehat{(}_{(0,0)}\right.$ has the structure of an $\widehat{\mathcal{O}}_{\mathcal{U},(0,0)} \llbracket z \rrbracket$-module;

(2) we have an isomorphism of (finite, free) $\widehat{\mathcal{O}}_{\mathcal{U},(0,0)} \llbracket z \rrbracket$-modules

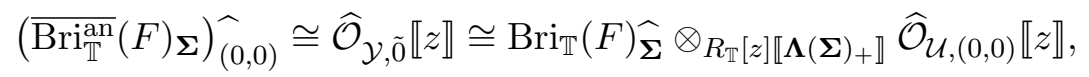

where $\widehat{\mathcal{O}}_{\mathcal{U},(0,0)}$ and $\widehat{\mathcal{O}}_{\mathcal{Y}, 0}$ are as in Lemma 4.18. In Part $(2)$, the $\widehat{\mathcal{O}}_{\mathcal{U},(0,0)} \llbracket z \rrbracket$-module structure on $\widehat{\mathcal{O}}_{\mathcal{Y}, \tilde{0}} \llbracket z \rrbracket$ is defined similarly to Definition 4.21: $\chi_{i}$ acts on it by the formula (4.8) and functions in $q$ and $z$ act in the usual way (see also Remark 4.23).

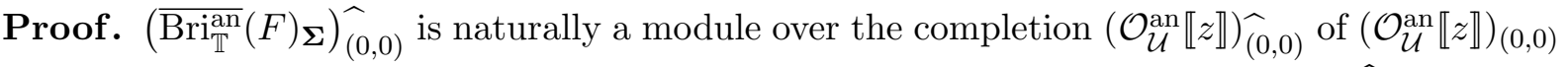
with respect to $\mathfrak{m}_{(0,0)}^{\text {an }}$. Hence Part $(1)$ follows from the fact ${ }^{13}$ that $\left(\mathcal{O}_{\mathcal{U}}^{\text {an }} \llbracket z \rrbracket\right)_{(0,0)}=\widehat{\mathcal{O}}_{\mathcal{U},(0,0)} \llbracket z \rrbracket$.

We show that the natural maps

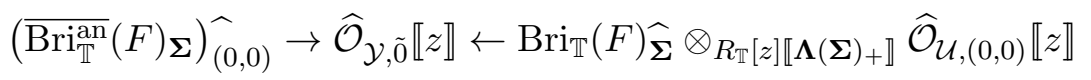

are isomorphisms, where the first map is induced by the map

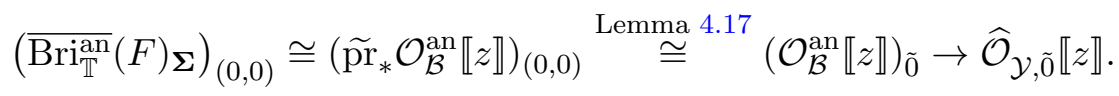

That the maps in (4.9) are isomorphisms follows from the following two facts: (a) all three modules in (4.9) are finite and free as $\widehat{\mathcal{O}}_{\mathcal{U},(0,0)} \llbracket z \rrbracket$-modules, and (b) the maps in (4.9) are isomorphisms modulo $z$. We already know that (b) holds by Lemma 4.18. In fact, the maps in (4.9) reduces to the isomorphisms in (4.5) modulo $z$. Thus we only need to show (a). Proposition 4.24 implies that $\left(\overline{\operatorname{Bri}_{\mathbb{T}}^{2 n}}(F)_{\Sigma}\right)_{(0,0)}$ is a finite free $\left(\mathcal{O}_{\mathcal{U}} \llbracket z \rrbracket\right)_{(0,0)}$-module, and thus $\left(\overline{\operatorname{Bri}_{\mathbb{T}}^{a n}}(F)_{\boldsymbol{\Sigma}}\right)_{(0,0)}$ is a finite free $\left(\mathcal{O}_{\mathcal{U}} \llbracket z \rrbracket\right)_{(0,0)}=\widehat{\mathcal{O}}_{\mathcal{U},(0,0)} \llbracket z \rrbracket$-module. Also, since $\operatorname{Bri}_{\mathbb{T}}(F)_{\boldsymbol{\Sigma}}$ is a finite free $R_{\mathbb{T}}[z] \llbracket \boldsymbol{\Lambda}(\boldsymbol{\Sigma})_{+} \rrbracket$ module, the third term in $(4.9)$ is a finite free $\widehat{\mathcal{O}}_{\mathcal{U},(0,0)}$-module. The finite-freeness of $\widehat{\mathcal{O}}_{\mathcal{Y}, \tilde{0}} \llbracket z \rrbracket$

\footnotetext{
${ }^{13}$ Note however that $\left(\mathcal{O}_{\mathcal{U}}^{\text {an }} \llbracket z \rrbracket\right)_{(0,0)} \neq \mathcal{O}_{\mathcal{U},(0,0)}^{\text {an }} \llbracket z \rrbracket$.
} 
follows from a discussion parallel to the proof of Proposition 4.24. Indeed, we know from Corollary 4.19 that $\widehat{\mathcal{O}}_{\mathcal{Y}, \tilde{0}}$ is a finite free $\widehat{\mathcal{O}}_{\mathcal{U},(0,0)}$-module, and any basis of $\widehat{\mathcal{O}}_{\mathcal{Y}, \tilde{0}}$ over $\widehat{\mathcal{O}}_{\mathcal{U},(0,0)}$ gives rise to a basis of $\widehat{\mathcal{O}}_{\mathcal{Y}, \tilde{0}} \llbracket z \rrbracket$ over $\widehat{\mathcal{O}}_{\mathcal{U},(0,0)} \llbracket z \rrbracket$. Part (2) is proved.

By restricting the above isomorphism to $\mathcal{V}=\mathcal{U} \cap(\mathcal{M} \times\{0\})$ and using Proposition 4.11, we have

Corollary 4.26. $\left(\overline{\operatorname{Bri}^{a n}}(F)_{\boldsymbol{\Sigma}} \widehat{)_{0}} \cong \operatorname{Bri}(F)_{\boldsymbol{\Sigma}} \otimes_{\mathbb{C}[z] \llbracket \Lambda(\Sigma)_{+} \rrbracket} \widehat{\mathcal{O}}_{\mathcal{V}, 0} \llbracket z \rrbracket\right.$.

Remark 4.27 (cf. Remarks 4.4 and 4.14). As before, we can describe the analytified Brieskorn module as a twisted de Rham cohomology. The complex $\left(\Omega_{\mathcal{B} / \mathcal{M}}^{\bullet, a n} \llbracket z \rrbracket, z d+d F \wedge\right)$ can be identified with the Koszul complex associated with the action of $\chi_{1}, \ldots, \chi_{n}$ on $\mathcal{O}_{\mathcal{B}}^{\text {an }} \llbracket z \rrbracket$ given by (4.8). Since $\chi_{1}, \ldots, \chi_{n}$ form a regular sequence for $\mathcal{O}_{\mathcal{B}}^{\text {an } \llbracket z \rrbracket, ~ w e ~ h a v e ~}$

$$
H^{i}\left(\Omega_{\mathcal{B} / \mathcal{M}}^{\bullet, a n} \llbracket z \rrbracket, z d+d F \wedge\right)= \begin{cases}0, & i \neq n, \\ \mathcal{O}_{\mathcal{B}}^{\operatorname{an}} \llbracket z \rrbracket /\left(\chi_{1}, \ldots, \chi_{n}\right) \mathcal{O}_{\mathcal{B}}^{\operatorname{an}} \llbracket z \rrbracket, & i=n,\end{cases}
$$

where the $n$th cohomology sheaf is supported on $\widetilde{\operatorname{pr}}^{-1}(\mathcal{M} \times\{0\}) \cap \mathcal{B}=\left\{u \in \mathcal{B}: x_{1} \frac{\partial F}{\partial x_{1}}(u)=\right.$ $\left.\cdots=x_{n} \frac{\partial F}{\partial x_{n}}(u)=0\right\}$. Therefore we have

$$
\overline{\operatorname{Bri}^{\mathrm{an}}}(F)_{\Sigma} \cong \operatorname{pr}_{*} H^{n}\left(\Omega_{\mathcal{B} / \mathcal{M}}^{\bullet, \text { an }} \llbracket z \rrbracket, z d+d F \wedge\right) \cong R^{n} \operatorname{pr}_{*}\left(\Omega_{\mathcal{B} / \mathcal{M}}^{\bullet, \text { an }} \llbracket z \rrbracket, z d+d F \wedge\right) .
$$

Note that the second and the third term is supported on $\mathcal{V}=\mathcal{U} \cap(\mathcal{M} \times\{0\})$.

\subsubsection{The higher residue pairing on the analytified Brieskorn module}

A version of K. Saito's higher residue pairing [96] on the completed equivariant Brieskorn module $\operatorname{Bri}_{\mathbb{T}}(F)_{\boldsymbol{\Sigma}}$ was introduced in [27, Section 6] via the asymptotic expansions of oscillatory integrals. We explain that the definition there can be extended to the analytified Brieskorn module $\overline{\operatorname{Bri}_{\mathbb{T}}^{a n}}(F)_{\Sigma}$. Consider the equivariant potential function (as appeared in Remark 4.4)

$$
F_{\mathbb{T}}=F_{\mathbb{T}}(x, q)=F(x, q)-\sum_{i=1}^{n} \chi_{i} \log x_{i} .
$$

This is a multi-valued function on $\mathcal{Y}$ with parameter $\chi \in \operatorname{Lie} \mathbb{T}$. For a fixed $(q, \chi) \in \mathcal{M}_{\mathbb{T}}=$ $\mathcal{M} \times \operatorname{Lie} \mathbb{T}$, (logarithmic) critical points of $\left.F_{\mathbb{T}}\right|_{\mathrm{pr}^{-1}(q)}$ are solution to the equation

$$
x_{i} \frac{\partial F}{\partial x_{i}}=\chi_{i}
$$

Thus we can regard $\widetilde{\text { pr }}: \mathcal{B} \rightarrow \mathcal{U}(4.3)$ as a family ${ }^{14}$ of critical points of $F_{\mathbb{T}}$. It follows from the study [27, Lemma 6.2] of critical points near $0_{\Sigma}$ that the fibre of the finite morphism $\left.\widetilde{p r}\right|_{\mathcal{B}}: \mathcal{B} \rightarrow \mathcal{U}$ at a generic point consists of $\operatorname{dim} H_{\mathrm{CR}}^{*}\left(\mathfrak{X}_{\boldsymbol{\Sigma}}\right)$ many reduced points. We write

$$
\mathcal{U}^{\mathrm{ss}}=\left\{(q, \chi) \in \mathcal{U}: \widetilde{\mathrm{pr}}^{-1}(q, \chi) \cap \mathcal{B} \text { consists of only reduced points }\right\} \neq \varnothing,
$$

where "ss" means semisimplicity. The complement of $\mathcal{U}^{\text {ss }}$ in $\mathcal{U}$ (called caustic) is an analytic subvariety in $\mathcal{U}$. Let $\phi \cdot \omega \in \overline{\operatorname{Bri}_{\mathbb{T}}^{\text {an }}}(F)_{\boldsymbol{\Sigma}}$ be a section over $\mathcal{U}^{\text {ss }}$, where $\phi=\phi(x, q, \chi) \in \widetilde{\operatorname{pr}}_{*}\left(\mathcal{O}_{\mathcal{B}}^{\text {an }} \llbracket z \rrbracket\right)$. For $(q, \chi) \in \mathcal{U}^{\text {ss }}$ and a critical point $p \in \widetilde{\mathrm{pr}}^{-1}(q, \chi) \cap \mathcal{B}$, we can define the formal asymptotic expansion of the oscillatory integral (see [27, Section 6.2])

$$
\int_{\Gamma(p)} e^{F_{\mathbb{T}} / z} \phi(x, q, \chi) \omega \sim e^{F_{\mathbb{T}}(p) / z}(-2 \pi z)^{n / 2} \sum_{n=0}^{\infty} a_{n}(q, \chi) z^{n} \quad \text { as } z \rightarrow 0 .
$$

\footnotetext{
${ }^{14}$ More precisely, $\mathcal{B}$ is the union of branches of critical points that tend to $\tilde{0}$ as $q \rightarrow 0=0 \Sigma$.
} 
We obtain the right-hand side by expanding the integrand $e^{F_{\mathbb{T}} / z} \phi(x, q, \chi)$ in Taylor series at $p$ (with respect to the logarithmic co-ordinates $\log x_{1}, \ldots, \log x_{n}$ ) and performing termwise (Gaussian) integration. More precisely, we have

$$
\sum_{n=0}^{\infty} a_{n}(q, \chi) z^{n}=\frac{1}{\left|\mathbf{N}_{\text {tor }}\right| \sqrt{\operatorname{det}\left(h_{i, j}\right)}}\left[e^{-\frac{z}{2} \sum_{i, j} h^{i, j} \frac{\partial}{\partial s_{i}} \frac{\partial}{\partial s_{j}}} e^{F_{\mathbb{T}}^{\geq 3} / z} \phi\left(p e^{s}, q, \chi\right)\right]_{s=0},
$$

where $s_{1}, \ldots, s_{n}$ are the logarithmic co-ordinates centred at $p$ so that $x_{j}=x_{j}(p) e^{s_{j}}$,

$$
h_{i, j}=\frac{\partial^{2} F_{\mathbb{T}}}{\partial \log x_{i} \partial \log x_{j}}(p)
$$

is the Hessian matrix at $p,\left(h^{i, j}\right)$ are the coefficients of the matrix inverse to $\left(h_{i, j}\right)$ and

$$
F_{\mathbb{T}}^{\geq 3}=\sum_{k \geq 3} \frac{1}{k !} \sum_{i_{1}, \ldots, i_{k}} \frac{\partial^{k} F_{\mathbb{T}}}{\partial \log x_{i_{1}} \cdots \partial \log x_{i_{k}}}(p) s_{i_{1}} \cdots s_{i_{k}}
$$

is the truncated Taylor expansion of $F_{\mathbb{T}}$ at the critical point $p$.

Definition 4.28. We define $\operatorname{Asym}_{p}(\phi \cdot \omega)$ to be the right-hand side of (4.10).

\section{Remark 4.29.}

(1) When the critical point $p$ does not lie in the logarithmic locus of $\mathcal{Y}$ and $\phi(x, q, \chi)$ is a polynomial, the above formal asymptotic expansion makes sense as an actual asymptotic expansion: for this we choose the integration cycle $\Gamma(p) \subset \mathcal{Y}_{q}:=\operatorname{pr}^{-1}(q)$ to be a stable manifold for the Morse function $x \mapsto \Re\left(F_{\mathbb{T}}(x, q)\right)$ associated with $p$ and assume that $z$ approaches zero from the negative real axis.

(2) More precisely, the above formal asymptotic expansion depends on the choice of the square root of the Hessian. This corresponds to the choice of an orientation of the Morse cycle $\Gamma(p)$ and a branch of $(-2 \pi z)^{n / 2}$.

Definition 4.30. The higher residue pairing of two sections $s_{1}, s_{2} \in \overline{\operatorname{Bri}_{\mathbb{T}}^{\mathrm{an}}}(F)_{\boldsymbol{\Sigma}}$ are defined as:

$$
P\left(s_{1}, s_{2}\right)(q, \chi)=\sum_{p \in \widetilde{\mathrm{pr}}^{-1}(q, \chi)}\left[\operatorname{Asym}_{p}\left(s_{1}\right)\right]_{z \rightarrow-z} \cdot \operatorname{Asym}_{p}\left(s_{2}\right),
$$

where $(q, \chi) \in \mathcal{U}^{\mathrm{ss}}$.

The higher residue pairing gives a bilinear pairing

$$
P:\left.\overline{\operatorname{Bri}_{\mathbb{T}}^{a n}}(F)_{\boldsymbol{\Sigma}}\right|_{\mathcal{U}^{\text {ss }}} \times\left.\overline{\operatorname{Bri}_{\mathbb{T}}^{\mathrm{an}}}(F)_{\boldsymbol{\Sigma}}\right|_{\mathcal{U}^{\text {ss }}} \rightarrow \mathcal{O}_{\mathcal{U}^{\mathrm{ss}}}^{\mathrm{an}} \llbracket z \rrbracket
$$

which satisfies the following properties [27, Proposition 6.8]:

(a) $P$ is $\mathcal{O}_{\mathcal{U}^{\text {ss }}}^{\text {an }}$-bilinear, non-degenerate, $z$-sesquilinear and symmetric:

$$
\begin{aligned}
& P\left(f(-z) s_{1}, s_{2}\right)=P\left(s_{1}, f(z) s_{2}\right)=f(z) P\left(s_{1}, s_{2}\right), \\
& P\left(s_{2}, s_{1}\right)=\left.P\left(s_{1}, s_{2}\right)\right|_{z \rightarrow-z},
\end{aligned}
$$

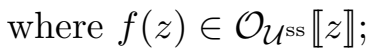

(b) $P$ is $\nabla$-flat, i.e., $d P\left(s_{1}, s_{2}\right)=P\left(\nabla s_{1}, s_{2}\right)+P\left(s_{1}, \nabla s_{2}\right)$; 
(c) $P$ is homogeneous of degree $-n\left(n=\operatorname{dim} \mathfrak{X}_{\boldsymbol{\Sigma}}\right)$, i.e.,

$$
\left(z \frac{\partial}{\partial z}+\mathcal{E}+n\right) P\left(s_{1}, s_{2}\right)=P\left(\operatorname{Gr} s_{1}, s_{2}\right)+P\left(s_{1}, \operatorname{Gr} s_{2}\right)
$$

where $\mathcal{E}$ is the Euler vector field (4.2).

(d) along $z=0, P$ equals the Grothendieck residue pairing.

Definition 4.31. Suppose that $\mathcal{V}^{\mathrm{ss}}:=\mathcal{U}^{\mathrm{ss}} \cap(\mathcal{M} \times\{0\})$ is nonempty. The non-equivariant higher residue pairing $P:\left.\overline{\operatorname{Bri}^{a n}}(F)_{\Sigma}\right|_{\mathcal{V}^{\text {ss }}} \times\left.{\overline{\mathrm{Bri}^{\mathrm{an}}}}(F)_{\boldsymbol{\Sigma}}\right|_{\mathcal{V}^{\mathrm{ss}}} \rightarrow \mathcal{O}_{\mathcal{V}}$ ss $\llbracket z \rrbracket$ is defined to be the restriction of the above $P$ to $\mathcal{V}^{\text {ss }}$. If $\mathfrak{X}_{\boldsymbol{\Sigma}}$ is compact, the argument in [62, Proposition 3.10] shows that $\mathcal{V}^{\text {ss }}$ is an open dense subset of $\mathcal{V}$, and hence the non-equivariant higher residue pairing is defined.

Remark 4.32. The definition of the higher residue pairing in terms of oscillatory integrals is originally due to Pham [90, 2éme Partie, 4].

Remark 4.33. The higher residue pairing here does not necessarily extend to the caustic $\mathcal{U} \backslash \mathcal{U}^{\text {ss }}$. Under mirror symmetry, the higher residue pairing corresponds to the Poincaré pairing [27, Theorem 6.11], therefore it has poles along $\chi=0$ when $\mathfrak{X}_{\Sigma}$ is noncompact. When $\mathfrak{X}_{\Sigma}$ is compact, it extends to a holomorphic and non-degenerate pairing in a neighbourhood of $\left(0_{\boldsymbol{\Sigma}}, 0\right)$.

\subsection{Analytic mirror isomorphism}

Using the convergence result [27, Theorem 7.2] (which generalizes [59, Theorem 1.2]), we show that the mirror isomorphism in Theorem 4.8 extends to a neighbourhood of $0=0_{\Sigma}$ as an isomorphism between the analytified Brieskorn module $\overline{\operatorname{Bri}_{\mathbb{T}}^{a n}}(F)_{\boldsymbol{\Sigma}}$ and the analytic quantum D-module. Let $\mathcal{U} \subset \mathcal{M}_{\mathbb{T}}$ be an open neighbourhood of $\left(0_{\boldsymbol{\Sigma}}, 0\right)$ as in Corollary 4.20. Recall that $\overline{\operatorname{Bri}_{\mathbb{T}}^{\mathrm{an}}}(F)_{\boldsymbol{\Sigma}}$ was defined on $\mathcal{U}$.

The mirror isomorphism in Theorem 4.8 induces, via the isomorphism in Proposition 4.25, the following isomorphism

$$
\widehat{\operatorname{Mir}}:\left(\overline{\operatorname{Bri}_{\mathbb{T}}^{\text {an }}}(F)_{\boldsymbol{\Sigma}}{\widehat{\left(0_{\Sigma}, 0\right)}} \cong \operatorname{mir}^{*} \operatorname{QDM}_{\mathbb{T}}\left(\mathfrak{X}_{\boldsymbol{\Sigma}}\right) \otimes_{R_{\mathbb{T}}[z] \llbracket \Lambda(\boldsymbol{\Sigma})_{+} \rrbracket} \widehat{\mathcal{O}}_{\mathcal{U},\left(0_{\boldsymbol{\Sigma}}, 0\right)} \llbracket z \rrbracket .\right.
$$

This isomorphism extends to an analytic neighbourhood of $\left(0_{\boldsymbol{\Sigma}}, 0\right)$. We recall the following facts from [27, Section 7]:

(a) the structure constants of the big equivariant quantum product are convergent and analytic in $q, \tau^{\prime}$ and $\chi$ [27, Corollary 7.3] (where $q$ and $\tau^{\prime}$ are parameters of the Kähler moduli space, see Section 2.2, and $\chi$ is the equivariant parameter);

(b) choose a Gr-homogeneous basis $\left\{\Omega_{i}\right\}_{i=0}^{s}$ of the completed Brieskorn module consisting of algebraic differential forms (i.e., $\Omega_{i} \in \mathbb{C}[z]\left[\mathbb{O}(\boldsymbol{\Sigma})_{+}\right] \omega$ ) and let $\left\{\phi_{i}\right\}_{i=0}^{s}$ be the basis of $H_{\mathrm{CR}}^{*}\left(\mathfrak{X}_{\boldsymbol{\Sigma}}\right)$ as in Section 2.1; then the matrix entries $M_{i}^{j}(q, \chi, z)$ of the mirror isomorphism Mir given by

$$
\operatorname{Mir}\left(\Omega_{i}\right)=\sum_{j=0}^{s} M_{i}^{j}(q, \chi, z) \phi_{j}
$$

belong to $\mathcal{O}^{\text {an }}\left(\mathcal{U}^{\prime}\right) \llbracket z \rrbracket$ for some open neighbourhood $\mathcal{U}^{\prime}$ of $\left(0_{\Sigma}, 0\right)$ in $\mathcal{M}_{\mathbb{T}}=\mathcal{M} \times \operatorname{Lie} \mathbb{T}[27$, Theorem 7.1];

(c) the mirror map mir is also analytic in a neighbourhood of $\left(0_{\boldsymbol{\Sigma}}, 0\right) \in \mathcal{M}_{\mathbb{T}}($ ibid. $)$. 
By Part (a), the equivariant quantum D-module $\mathrm{QDM}_{\mathbb{T}}\left(\mathfrak{X}_{\boldsymbol{\Sigma}}\right)$ (see $(2.10)$ ) extends to a small analytic neighbourhood $U$ of $q=\tau^{\prime}=\chi=0$ in the equivariant Kähler moduli space

$$
\left[\mathcal{M}_{\mathrm{A}, \mathbb{T}}\left(\mathfrak{X}_{\boldsymbol{\Sigma}}\right) / \operatorname{Pic}^{\mathrm{st}}\left(\mathfrak{X}_{\boldsymbol{\Sigma}}\right)\right]
$$

(see $(2.5))$. We denote it by

$$
\mathrm{QDM}_{\mathbb{T}}^{\mathrm{an}}\left(\mathfrak{X}_{\boldsymbol{\Sigma}}\right):=\left(H_{\mathrm{CR}, \mathbb{T}}^{*}\left(\mathfrak{X}_{\boldsymbol{\Sigma}}\right) \otimes \mathcal{O}_{\widetilde{U}}^{\mathrm{an}}[z], \nabla, \mathrm{Gr}, P\right),
$$

where $\widetilde{U}$ is the preimage of $U$ in $\mathcal{M}_{\mathrm{A}, \mathbb{T}}\left(\mathfrak{X}_{\boldsymbol{\Sigma}}\right) ; H_{\mathrm{CR}, \mathbb{T}}^{*}\left(\mathfrak{X}_{\boldsymbol{\Sigma}}\right) \otimes \mathcal{O}_{\widetilde{U}}^{\text {an }}[z]$ is a $\operatorname{Pic}^{\text {st }}\left(\mathfrak{X}_{\boldsymbol{\Sigma}}\right)$-equivariant sheaf by the Galois symmetry in Section 2.2, and we regard it as a sheaf on the stack $U=$ $\left[\widetilde{U} / \operatorname{Pic}^{\text {st }}\left(\mathfrak{X}_{\boldsymbol{\Sigma}}\right)\right]$. By Part (b), $\widehat{\operatorname{Mir}}^{-1}\left(\phi_{i}\right)$ extends to a section of $\overline{\operatorname{Bri}_{\mathbb{T}}^{\text {an }}}(F)_{\boldsymbol{\Sigma}}$ over a small analytic neighbourhood $\mathcal{U}^{\prime} \subset \mathcal{U}$ of $\left(0_{\boldsymbol{\Sigma}}, 0\right) \in \mathcal{M}_{\mathbb{T}}$. By Part (c), by shrinking $\mathcal{U}^{\prime}$ if necessary, we have an analytic mirror map mir: $\mathcal{U}^{\prime} \rightarrow U$. We now have the following result.

Theorem 4.34. The isomorphism $\widehat{\mathrm{Mir}}$ in (4.12) extends to an open neighbourhood $\mathcal{U}^{\prime} \subset \mathcal{U}$ of $\left(0_{\boldsymbol{\Sigma}}, 0\right) \in \mathcal{M}_{\mathbb{T}}=\mathcal{M} \times \operatorname{Lie} \mathbb{T}$ and yields an isomorphism $\mathrm{Mir}^{\text {an }}$ of $\mathcal{O}_{\mathcal{U}^{\prime}}^{\text {an }} \llbracket z \rrbracket$-modules:

$$
\operatorname{Mir}^{\text {an }}:\left.\overline{\operatorname{Bri}_{\mathbb{T}}^{\text {an }}}(F)_{\Sigma}\right|_{\mathcal{U}^{\prime}} \cong \operatorname{mir}^{*} \overline{\operatorname{QDM}_{\mathbb{T}}^{\text {an }}}\left(\mathfrak{X}_{\boldsymbol{\Sigma}}\right),
$$

where $\overline{\mathrm{QDM}_{\mathbb{T}}^{\mathrm{an}}}\left(\mathfrak{X}_{\boldsymbol{\Sigma}}\right)$ denotes the z-adic completion of $\mathrm{QDM}_{\mathbb{T}}^{\mathrm{an}}\left(\mathfrak{X}_{\boldsymbol{\Sigma}}\right)$, i.e.,

$$
\overline{\mathrm{QDM}_{\mathbb{T}}^{\mathrm{an}}}\left(\mathfrak{X}_{\Sigma}\right):=\mathrm{QDM}_{\mathbb{T}}^{\mathrm{an}}\left(\mathfrak{X}_{\Sigma}\right) \otimes_{\mathcal{O}_{U}^{\mathrm{an}}[z]} \mathcal{O}_{U}^{\mathrm{an}} \llbracket z \rrbracket .
$$

The analytic mirror isomorphism Mir $^{\text {an }}$ satisfies the same properties (1)-(3) as in Theorem 4.8 (see Section 4.3.3 for the higher residue pairing on $\overline{\operatorname{Bri}_{\mathbb{T}}^{a n}}(F)_{\Sigma}$ ).

Remark 4.35. As explained before Theorem 4.8, in the above theorem, we choose a splitting $\mathbf{N} \rightarrow \mathbb{O}^{\boldsymbol{\Sigma}}$ of the refined fan sequence (3.12), which simultaneously defines a partial connection $\nabla$ on $\overline{\operatorname{Bri}_{\mathbb{T}}^{\mathrm{an}}}(F)_{\boldsymbol{\Sigma}}$ and the equivariant quantum D-module.

Remark 4.36. The analytic mirror theorem above shows that the analytified Brieskorn module can be further analytified in the $z$-direction ( this as a solution to the Birkhoff problem (i.e., finding a normal form of the Gauss-Manin connection) which has been studied extensively in the construction of K. Saito's flat structure $[11,37,38,93,97,98]$.

\section{Discrepant wall-crossings}

We study the change of quantum cohomology of smooth toric DM stacks under a "discrepant" wall crossing. We show a decomposition of formal (i.e., completed in the variable $z$ ) quantum D-modules under discrepant wall-crossings. We work in the set-up of Section 3.1 and fix the data $(\mathbf{N}, \Pi, S)$ as usual.

\subsection{Discrepant transformation of smooth toric DM stacks}

We describe birational transformations between smooth toric DM stacks following [45], [15, Sections 4 and 5], [31, Section 5.1 and 6.3] and [5, Section 3].

Recall from Section 3.1.1 that the toric stacks $\mathfrak{X}_{\boldsymbol{\Sigma}}$ with $\boldsymbol{\Sigma} \in \mathfrak{F a n}(S)$ arise as the GIT quotients of $\mathbb{C}^{S}$ by the torus $\mathbb{L}_{\mathbb{C}^{\times}}$. These toric stacks are birational to each other since they contain the (stacky) torus $\left[\left(\mathbb{C}^{\times}\right)^{S} / \mathbb{L}_{\mathbb{C}^{\times}}\right]$as an open dense subset. We can regard $\mathbb{L}_{\mathbb{R}}^{\star}$ as the space of GIT

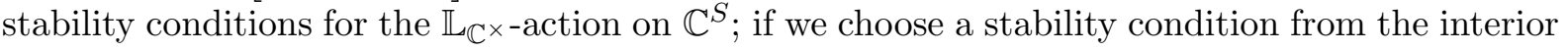
of the maximal cone $\operatorname{cpl}(\boldsymbol{\Sigma})$ of the secondary fan $\Xi$ (see Definition 3.6), then the corresponding 
GIT quotient is $\mathfrak{X}_{\boldsymbol{\Sigma}}$. We choose two adjacent maximal cones $\operatorname{cpl}\left(\boldsymbol{\Sigma}_{+}\right), \operatorname{cpl}\left(\boldsymbol{\Sigma}_{-}\right)$of $\boldsymbol{\Xi}$ which are separated by a hyperplane wall $W \subset \mathbb{L}_{\mathbb{R}}^{\star}$. Here we assume that $W \cap \operatorname{cpl}\left(\boldsymbol{\Sigma}_{+}\right)=W \cap \operatorname{cpl}\left(\boldsymbol{\Sigma}_{-}\right)$ is a common codimension-one face of $\operatorname{cpl}\left(\boldsymbol{\Sigma}_{ \pm}\right)$. Let $\mathbf{w} \in \mathbb{L}$ be a primitive integral vector which is perpendicular to the wall $W \subset \mathbb{L}_{\mathbb{R}}^{\star}$ and is non-negative on the chamber $\operatorname{cpl}\left(\boldsymbol{\Sigma}_{+}\right)$. By the definition of $\mathbb{L}$ (see (3.3)), the vector $\mathbf{w} \in \mathbb{L}$ gives rise to a linear relation

$$
\sum_{b \in S}\left(D_{b} \cdot \mathbf{w}\right) b=0
$$

where recall that $D_{b}=D\left(e_{b}^{\star}\right)$ (see (3.4)). This linear relation defines a circuit $\left\{b \in S: D_{b} \cdot \mathbf{w} \neq 0\right\}$ in the terminology of Gelfand-Kapranov-Zelevinsky [45], where a 'circuit' means a minimal linearly dependent set. The transition between $\boldsymbol{\Sigma}_{+}$and $\boldsymbol{\Sigma}_{-}$can be described in terms of the circuit ('modification along a circuit' [45]).
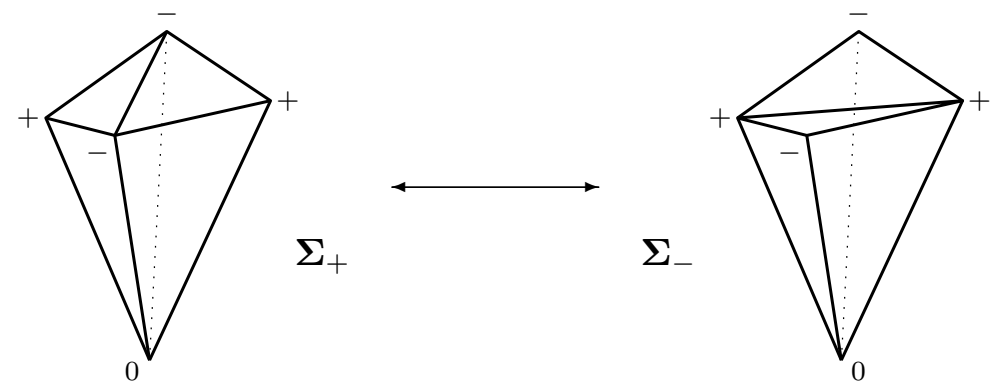

Figure 5. Modification along a circuit. The signs \pm mean rays belonging to $M_{ \pm}=\left\{b \in S: \pm D_{b} \cdot \mathbf{w}>0\right\}$.

For $I \subset S$, we write ${ }^{15}$

$$
\sigma_{I}:=\sum_{b \in I} \mathbb{R}_{\geq 0} \bar{b} \subset \mathbf{N}_{\mathbb{R}}, \quad \angle_{I}:=\sum_{b \in I} \mathbb{R}_{>0} D_{b} \subset \mathbb{L}_{\mathbb{R}}^{\star}
$$

We also set $\sigma_{\varnothing}=\{0\}, \angle_{\varnothing}=\{0\}$. Consider the (not necessarily simplicial) fan $\Sigma_{0}$ on the vector space $\mathbf{N}_{\mathbb{R}}$ given by $\Sigma_{0}:=\left\{\sigma_{I}: I \in \mathscr{S}_{0}\right\}$, where

$$
\mathscr{S}_{0}=\left\{I \subset S: L_{S \backslash I} \text { contains the relative interior of } \operatorname{cpl}\left(\boldsymbol{\Sigma}_{+}\right) \cap \operatorname{cpl}\left(\boldsymbol{\Sigma}_{-}\right)\right\} \text {. }
$$

The simplicial fans $\Sigma_{ \pm}$underlying $\Sigma_{ \pm}$arise as different subdivisions of $\Sigma_{0}$. Set $M_{ \pm}=\{b \in S$ : $\left.\pm D_{b} \cdot \mathbf{w}>0\right\}$. We have a decomposition $\mathscr{S}_{0}=\mathscr{S}_{0}^{\text {simp }} \sqcup \mathscr{S}_{0}^{\text {circ }}$ (disjoint union), where

$$
\begin{aligned}
& \mathscr{S}_{0}^{\text {simp }}=\left\{I \in \mathscr{S}_{0}: M_{+} \not \subset I, M_{-} \not \subset I\right\}, \\
& \mathscr{S}_{0}^{\text {circ }}=\left\{I \in \mathscr{S}_{0}: M_{+} \cup M_{-} \subset I\right\},
\end{aligned}
$$

such that $\Sigma_{ \pm}=\left\{\sigma_{I}: I \in \mathscr{S}_{ \pm}\right\}$with

$$
\mathscr{S}_{ \pm}=\left\{I: I \in \mathscr{S}_{0}^{\text {simp }}\right\} \sqcup\left\{I \backslash J: I \in \mathscr{S}_{0}^{\text {circ }}, \varnothing \neq J \subset M_{ \pm}\right\} .
$$

The set of rays of $\boldsymbol{\Sigma}_{ \pm}$is given by $R_{ \pm}:=R\left(\boldsymbol{\Sigma}_{ \pm}\right)=\bigcup_{I \in \mathscr{S}_{ \pm}} I$. See [31, Lemma 5.2 $]^{16}$. Here $I \in \mathscr{S}_{0}^{\text {simp }}$ yields a simplicial cone $\sigma_{I} \in \Sigma_{0}$ belonging to both $\Sigma_{+}$and $\Sigma_{-}$, and $I \in \mathscr{S}_{0}^{\text {circ }}$ yields a (not necessarily simplicial) cone $\sigma_{I} \in \Sigma_{0}$ containing the circuit $M_{+} \cup M_{-}$; the cone $\sigma_{I}$ with $I \in \mathscr{S}_{0}^{\text {circ }}$ is subdivided into simplicial cones $\sigma_{I \backslash\{v\}}, v \in M_{ \pm}$in the fans $\Sigma_{ \pm}$. See Fig. 5 . We also

\footnotetext{
${ }^{15}$ Note that $\sigma_{I}$ is a closed cone, whereas $\angle_{I}$ is a relatively open cone.

${ }^{16}$ In [31, Section 5.1], the set of "anti-cones" $\left\{S \backslash I: I \in \mathscr{S}_{0}\right\},\left\{S \backslash I: I \in \mathscr{S}_{0}^{\text {simp }}\right\},\left\{S \backslash I: I \in \mathscr{S}_{0}^{\text {circ }}\right\}$ are denoted by $\mathcal{A}_{0}, \mathcal{A}_{0}^{\text {thick }}, \mathcal{A}_{0}^{\text {thin }}$ respectively.
} 
remark that $M_{+} \cup M_{-} \in \mathscr{S}_{0}^{\text {circ }}$ so that $\left(M_{+} \cup M_{-}\right) \backslash\{v\} \in \mathscr{S}_{ \pm}$for every $v \in M_{ \pm}$. (In particular, $M_{ \pm} \subset R_{\mp .}$ )

Let $\mathfrak{X}_{ \pm}$denote the toric stack corresponding to $\boldsymbol{\Sigma}_{ \pm}$. As discussed in [15, Section 5], [31, Section 6.3], the toric birational map $\varphi: \mathfrak{X}_{+}-\rightarrow \mathfrak{X}_{-}$fits into a commutative diagram

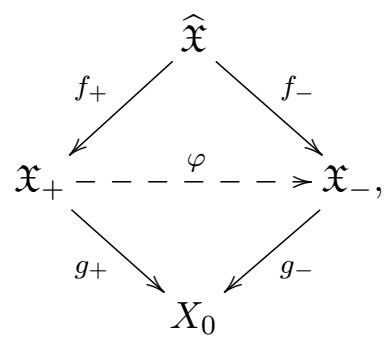

where $X_{0}$ is the toric variety associated with $\Sigma_{0}, \widehat{\mathfrak{X}}$ is another smooth toric DM stack and $f_{ \pm}: \widehat{\mathfrak{X}} \rightarrow \mathfrak{X}_{ \pm}, g_{ \pm}: \mathfrak{X}_{ \pm} \rightarrow X_{0}$ are projective birational toric morphisms. Define $\hat{b} \in \mathbf{N} \cap \Pi$ to be the vector:

$$
\hat{b}:=\sum_{D_{b} \cdot \mathbf{w}>0}\left(D_{b} \cdot \mathbf{w}\right) b=-\sum_{D_{b} \cdot \mathbf{w}<0}\left(D_{b} \cdot \mathbf{w}\right) b .
$$

The smooth toric DM stack $\widehat{\mathfrak{X}}$ is given by a stacky fan $\widehat{\boldsymbol{\Sigma}}$ adapted to $S \cup\{\hat{b}\}$ (in the sense of Definition 3.3): the set of rays of $\widehat{\Sigma}$ is $\widehat{R}=\left(R_{+} \cap R_{-}\right) \cup\{\hat{b}\}$ where $R_{ \pm}:=R\left(\boldsymbol{\Sigma}_{ \pm}\right)$; the fan $\widehat{\Sigma}$ underlying $\widehat{\boldsymbol{\Sigma}}$ is a simultaneous subdivision of $\Sigma_{+}$and $\Sigma_{-}$given by

$$
\widehat{\Sigma}=\left\{\sigma_{I}: I \in \mathscr{S}_{0}^{\text {simp }}\right\} \sqcup\left\{\sigma_{K}: K=I \backslash\left(J_{+} \cup J_{-}\right) \cup\{\hat{b}\}, I \in \mathscr{S}_{0}^{\text {circ }}, \varnothing \neq J_{ \pm} \subset M_{ \pm}\right\} .
$$

The toric morphisms $f_{ \pm}: \widehat{\mathfrak{X}} \rightarrow \mathfrak{X}_{ \pm}$are induced by natural maps $\widehat{\boldsymbol{\Sigma}} \rightarrow \boldsymbol{\Sigma}_{ \pm}$of stacky fans. We refer the reader to [31, Section 6.3] for a description of $\widehat{\mathfrak{X}}$ and $f_{ \pm}$in terms of GIT quotients.

Lemma 5.1. Let $K_{ \pm}=K_{\mathfrak{X}_{ \pm}}$denote the canonical class of $\mathfrak{X}_{ \pm}$and $E \subset \widehat{\mathfrak{X}}$ denote the toric divisor of $\widehat{\mathfrak{X}}$ corresponding to the ray $\hat{b}$. Then we have

$$
f_{+}^{\star} K_{+}=f_{-}^{\star} K_{-}+\left(\sum_{b \in S} D_{b} \cdot \mathbf{w}\right)[E] .
$$

Proof. This follows immediately from [31, Proposition 6.21].

In view of the lemma above, we say that the birational transformation $\mathfrak{X}_{+}-\rightarrow \mathfrak{X}_{-}$is crepant if $\sum_{b \in S} D_{b} \cdot \mathbf{w}=0$ (i.e., $\sum_{b \in S} D_{b}$ is on the wall $W$ ) and discrepant otherwise. We shall restrict ourselves to the case where the transformation is discrepant. By exchanging $\mathfrak{X}_{+}$and $\mathfrak{X}_{-}$if necessary, we may assume:

Assumption 5.2. The birational transformation $\mathfrak{X}_{+} \rightarrow \mathfrak{X}_{-}$satisfies $f_{+}^{\star} K_{+}>f_{-}^{\star} K_{-}$, i.e., $\sum_{b \in S} D_{b} \cdot \mathbf{w}>0$.

Remark 5.3. Under the above assumption, the dimension of orbifold cohomology decreases: $\operatorname{dim} H_{\mathrm{CR}}^{*}\left(\mathfrak{X}_{+}\right)>\operatorname{dim} H_{\mathrm{CR}}^{*}\left(\mathfrak{X}_{-}\right)$. We can see this from the change of the fans, and using the fact that $\left|\mathbf{N}_{\text {tor }}\right|^{-1} \operatorname{dim} H_{\mathrm{CR}}^{*}\left(\mathfrak{X}_{\boldsymbol{\Sigma}}\right)$ equals the sum of volumes of simplices spanned by $\{0\} \cup\{\bar{b} \in R(\boldsymbol{\Sigma})$ : $\bar{b} \in \sigma\}$ over all maximal cones $\sigma$ of $\boldsymbol{\Sigma}$, where we normalize the volume of the standard simplex to be one (see, e.g., [62, Lemma 3.9]). 
Remark 5.4. There are three types of discrepant wall-crossings: (I) $\mathfrak{X}_{+}$and $\mathfrak{X}_{-}$are isomorphic in codimension one ("flip"), (II) the birational map induces a map (i) $\mathfrak{X}_{+} \rightarrow X_{-}$or (ii) $\mathfrak{X}_{-} \rightarrow X_{+}$ contracting a divisor to a toric subvariety, where $X_{ \pm}$is the coarse moduli space of $\mathfrak{X}_{ \pm}$("discrepant resolution") and (III) $X_{+}=X_{-}$but the stack structures of $\mathfrak{X}_{+}$and $\mathfrak{X}_{-}$differ along a divisor. In terms of stacky fans, we have

(I) $R_{+}=R_{-}, \sharp M_{+} \geq 2$ and $\sharp M_{-} \geq 2$ (rays are the same);

(II-i) $R_{+}=R_{-} \sqcup M_{-}, \sharp M_{-}=1$ and $\sharp M_{+} \geq 2$ (removing one ray);

(II-ii) $R_{-}=R_{+} \sqcup M_{+}, \sharp M_{+}=1$ and $\sharp M_{-} \geq 2$ (adding one ray);

(III) $R_{+} \backslash R_{-}=M_{-}, R_{-} \backslash R_{+}=M_{+}$and $\sharp M_{-}=\sharp M_{+}=1$ (replace a ray $b_{-} \in R_{+}$with a shorter and parallel ray $b_{+} \in R_{-}$, where $M_{ \pm}=\left\{b_{ \pm}\right\}$).

This is similar to the classification of crepant transformations given in [31, Propositions 5.4 and 5.5] and can be shown by a parallel argument.

Example 5.5. Let $a_{1}, \ldots, a_{k}, b_{1}, \ldots, b_{l}$ be positive integers with $a_{1}+\cdots+a_{k}<b_{1}+\cdots+b_{l}$. Consider the $\mathbb{C}^{\times}$-action on $\mathbb{C}^{k+l}$ given by the weights $\left(-a_{1}, \ldots,-a_{k}, b_{1}, \ldots, b_{l}\right)$. The GIT variation gives a discrepant transformation between the spaces:

$$
\begin{aligned}
& \mathfrak{X}_{+}=\text {the total space of } \mathcal{O}\left(-a_{1}\right) \oplus \cdots \oplus \mathcal{O}\left(-a_{k}\right) \text { over } \mathbb{P}\left(b_{1}, \ldots, b_{l}\right) \text {, and } \\
& \mathfrak{X}_{-}=\text {the total space of } \mathcal{O}\left(-b_{1}\right) \oplus \cdots \oplus \mathcal{O}\left(-b_{l}\right) \text { over } \mathbb{P}\left(a_{1}, \ldots, a_{k}\right) .
\end{aligned}
$$

Following the classification in Remark 5.4, we have: (I) if $k, l \geq 2$, this is a flip; (II-i) if $k=1$ and $l \geq 2$, this is a resolution $\mathfrak{X}_{+} \rightarrow X_{-}=\mathbb{C}^{l} / \mu_{a_{1}}$ with positive discrepancy; (II-ii) if $k \geq 2$ and $l=1$, this is a resolution $\mathfrak{X}_{-} \rightarrow X_{+}=\mathbb{C}^{k} / \mu_{b_{1}}$ with negative discrepancy; (III) if $k=l=1$, we have $\mathfrak{X}_{+}=\left[\mathbb{C} / \mu_{b_{1}}\right]$ and $\mathfrak{X}_{-}=\left[\mathbb{C} / \mu_{a_{1}}\right]$; the stack structure at the origin changes. Note that the example in Section 3.6.3 is a special case of the current example with $k=2, l=1$, $\left(a_{1}, a_{2}, b_{1}\right)=(1,1, d)$.

Example 5.6. A blow-up along a toric subvariety is an example of type (II) discrepant transformation.

Example 5.7. A root construction [21] along a toric divisor is an example of type (III) discrepant transformation.

\subsection{The LG model along a curve}

Consider the partially compactified LG model $(\operatorname{pr}: \mathcal{Y} \rightarrow \mathcal{M}, F)$ from Section 3.2. Recall that $\mathcal{M}$ is defined in terms of the secondary fan $\Xi$ consisting of maximal cones $\operatorname{cpl}(\boldsymbol{\Sigma}), \boldsymbol{\Sigma} \in \mathfrak{F} \mathfrak{a n}(S)$. Let $\mathcal{C} \subset \mathcal{M}$ denote the 1-dimensional toric substack corresponding to the codimension-1 cone $\operatorname{cpl}\left(\boldsymbol{\Sigma}_{+}\right) \cap \operatorname{cpl}\left(\boldsymbol{\Sigma}_{+}\right)$. The curve $\mathcal{C}$ lies in the boundary of $\mathcal{M}$ and connects the large radius limit points $0_{\boldsymbol{\Sigma}_{+}}$and $0_{\boldsymbol{\Sigma}_{-}}$.

We cover $\mathcal{C}$ by the two open sets $\mathcal{M}_{ \pm}:=\mathcal{M}_{\boldsymbol{\Sigma}_{ \pm}}=\left[\operatorname{Spec} \mathbb{C}\left[\boldsymbol{\Lambda}\left(\boldsymbol{\Sigma}_{ \pm}\right)_{+}\right] / \operatorname{Pic}^{\mathrm{st}}\left(\mathfrak{X}_{ \pm}\right)\right]$; in these local charts, the embedding $\mathcal{C} \subset \mathcal{M}$ is given by the $\mathbb{C}$-algebra homomorphism:

$$
\mathbb{C}\left[\boldsymbol{\Lambda}\left(\boldsymbol{\Sigma}_{ \pm}\right)_{+}\right] \rightarrow \mathbb{C}\left[q^{ \pm \mathbf{w} / e_{ \pm}}\right], \quad q^{\lambda} \mapsto \begin{cases}q^{\lambda}, & \text { if } \lambda \text { is proportional to } \mathbf{w} \\ 0, & \text { otherwise }\end{cases}
$$

where $e_{ \pm} \in \mathbb{N}$ is the smallest common denominator of $\left\{c \in \mathbb{Q}: c \mathbf{w} \in \boldsymbol{\Lambda}\left(\boldsymbol{\Sigma}_{ \pm}\right)\right\}$. (Recall that $\boldsymbol{\Lambda}\left(\boldsymbol{\Sigma}_{ \pm}\right)_{+}=\boldsymbol{\Lambda}\left(\boldsymbol{\Sigma}_{ \pm}\right) \cap \operatorname{cpl}\left(\boldsymbol{\Sigma}_{ \pm}\right)^{\vee}$, see Lemma 3.14. $)$ 
Lemma 5.8. The inverse image $\mathrm{pr}^{-1}(\mathcal{C})$ is covered by two charts

$$
\operatorname{pr}^{-1}\left(\mathcal{C} \cap \mathcal{M}_{ \pm}\right)=\left[\operatorname{Spec}\left(A_{ \pm}\right) / \operatorname{Pic}^{\mathrm{st}}\left(\mathfrak{X}_{ \pm}\right)\right]
$$

where $A_{ \pm}$is the $\mathbb{C}\left[q^{ \pm \mathbf{w} / e_{ \pm}}\right]$-algebra $\bigoplus_{v \in \mathbf{N} \cap \Pi} \mathbb{C}\left[q^{ \pm \mathbf{w} / e_{ \pm}}\right] w_{v}^{ \pm}$equipped with the product

$$
w_{v_{1}}^{ \pm} w_{v_{2}}^{ \pm}= \begin{cases}q^{\Psi^{ \pm}\left(v_{1}\right)+\Psi^{ \pm}\left(v_{2}\right)-\Psi^{ \pm}\left(v_{1}+v_{2}\right)} w_{v_{1}+v_{2}}^{ \pm}, & \text {if } v_{1}, v_{2} \text { lie in the same cone of } \Sigma_{0} \\ 0 & \text { otherwise }\end{cases}
$$

where $\Psi^{ \pm}\left(v_{1}\right)+\Psi^{ \pm}\left(v_{2}\right)-\Psi^{ \pm}\left(v_{1}+v_{2}\right)$ is proportional to $\mathbf{w}$ in the first case, $w_{v}^{ \pm}$is the restriction of $u^{\left(\Psi^{ \pm}(\bar{v}), v\right)}$ to $\operatorname{pr}^{-1}\left(\mathcal{C} \cap \mathcal{M}_{ \pm}\right)$and $\Psi^{ \pm}:=\Psi^{\Sigma_{ \pm}}$(see Notation 3.10). The two charts are glued by

$$
w_{v}^{-}= \begin{cases}q^{\Psi^{-}(v)-\Psi^{+}(v)} w_{v}^{+}, & \text {if } v \text { lies in a cone } \sigma_{I} \text { with } I \in \mathscr{S}_{0}^{\text {circ }} \\ w_{v}^{+}, & \text {otherwise }\end{cases}
$$

Proof. The space $\operatorname{pr}^{-1}\left(\mathcal{C} \cap \mathcal{M}_{ \pm}\right)$is the base change of $\mathcal{Y}_{\boldsymbol{\Sigma}_{ \pm}}=\left[\operatorname{Spec} \mathbb{C}\left[\mathbb{O}\left(\boldsymbol{\Sigma}_{ \pm}\right)_{+}\right] / \operatorname{Pic}^{\text {st }}\left(\mathfrak{X}_{ \pm}\right)\right]$ via (5.3). The conclusion follows from Remark 3.17 and the description of cones of $\Sigma_{0}, \Sigma_{ \pm}$in terms of the circuit (5.1).

Remark 5.9. We describe how the curve $\mathcal{C}$ looks like in the Kähler moduli space. Using the equation (5.3) for $\mathcal{C}$ and the asymptotics of the mirror map (Remark 4.10), we find that the image of $\mathcal{C}$ under the (non-equivariant) mirror map for $\mathfrak{X}_{+}$is asymptotically close to, near the large radius limit point $0_{\boldsymbol{\Sigma}_{+}}$,

type (I) or (II-i) case: the curve given by $\tau^{\prime}=0$ and $q^{d}=0$ for all $d \in \Lambda_{+}^{\boldsymbol{\Sigma}_{+}} \backslash \mathbb{Q}_{\geq 0} \mathbf{W}$ (i.e., the curve corresponding to the extremal class $\left.\mathbf{w} \in H_{2}\left(\mathfrak{X}_{+}, \mathbb{Q}\right)\right)$;

type (II-ii) or (III) case: the curve given by $\tau^{\prime} \in \mathbb{C D}_{b_{+}}$and $q^{d}=0$ for all $d \in \boldsymbol{\Lambda}_{+}^{\boldsymbol{\Sigma}_{+}} \backslash\{0\}$, where $b_{+}$is the unique element of $M_{+}$and $\mathfrak{D}_{b_{+}} \in H_{\mathrm{CR}}^{<2}\left(\mathfrak{X}_{+}\right)$is as in Remark 4.10.

Here we use the notation on the Kähler moduli space from Section 2.2 and the classification in Remark 5.4. Similarly, near $0_{\boldsymbol{\Sigma}_{-}}$, the image of $\mathcal{C}$ under the mirror map for $\mathfrak{X}_{-}$is asymptotically close to

type (I) or (II-ii) case: the curve given by $\tau^{\prime}=0$ and $q^{d}=0$ for all $d \in \boldsymbol{\Lambda}_{+}^{\boldsymbol{\Sigma}_{-}} \backslash \mathbb{Q}_{\geq 0}(-\mathbf{w})$ (i.e., the curve corresponding to the extremal class $-\mathbf{w} \in H_{2}\left(\mathfrak{X}_{-}, \mathbb{Q}\right)$ );

type (II-i) or (III) case: the curve given by $\tau^{\prime} \in \mathbb{C D}_{b_{-}}$and $q^{d}=0$ for all $d \in \boldsymbol{\Lambda}_{+}^{\boldsymbol{\Sigma}_{-}} \backslash\{0\}$, where $b_{-}$is the unique element of $M_{-}$and $\mathfrak{D}_{b_{-}} \in H_{\mathrm{CR}}^{>2}\left(\mathfrak{X}_{-}\right)$is as in Remark 4.10.

The above classes $\mathfrak{D}_{b_{ \pm}}$are supported on the image of the exceptional divisor. We note that they can be zero, and in that case we need to examine the higher-order terms in the mirror map to see the asymptotic behaviour of $\mathcal{C}$.

Remark 5.10. Note that $\operatorname{pr}^{-1}(\mathcal{C}) \subset \mathcal{Y}$ is a possibly reducible toric substack and its components are in one-to-one correspondence with maximal cones of the fan $\Sigma_{0}$. The LG potential restricted to $\operatorname{pr}^{-1}(\mathcal{C})$ is of the form $F=\sum_{b \in R_{+} \cup R_{-}} u_{b}$ (here $u_{b}$ with $b \in S \backslash\left(R_{+} \cup R_{-}\right)$vanishes on $\operatorname{pr}^{-1}(\mathcal{C})$ ). 


\subsection{Decomposition of the Brieskorn module}

In this section, we show that the analytified Brieskorn module associated with $\boldsymbol{\Sigma}_{-}$is a direct summand of that associated with $\boldsymbol{\Sigma}_{+}$in a neighbourhood of $\mathcal{C}$. For this, we study the family (4.3)

$$
\widetilde{\operatorname{pr}}=\left(\operatorname{pr}, x_{1} \frac{\partial F}{\partial x_{1}}, \ldots, x_{n} \frac{\partial F}{\partial x_{n}}\right): \mathcal{Y} \rightarrow \mathcal{M}_{\mathbb{T}}=\mathcal{M} \times \operatorname{Lie} \mathbb{T}
$$

over an analytic neighbourhood of the curve $\mathcal{C} \times\{0\} \subset \mathcal{M} \times$ Lie $\mathbb{T}$, where $x_{1}, \ldots, x_{n}$ are coordinates along fibres of $\mathcal{Y} \rightarrow \mathcal{M}$ as in Section 4.1. Recall that this family can be regarded as the relative critical scheme of $F_{\mathbb{T}}$ (see Section 4.3.3).

The $\mathbb{C}^{\times}$-action generated by the Euler vector field (4.2) plays an important role in the following discussion. Consider the elements $\sum_{b \in S} e_{b}^{\star} \in\left(\mathbb{Z}^{S}\right)^{\star}$ and $\sum_{b \in S} D_{b} \in \mathbb{L}^{\star}$; they define $\mathbb{C}^{\times}$actions, respectively, on $\mathcal{Y}$ and $\mathcal{M}$ such that pr: $\mathcal{Y} \rightarrow \mathcal{M}$ is $\mathbb{C}^{\times}$-equivariant. In terms of the co-ordinates $\left(u_{b}\right)_{b \in S}$, the $\mathbb{C}^{\times}$-action is given by

$$
s \cdot u_{b}=s u_{b} \quad \text { with } s \in \mathbb{C}^{\times},
$$

and the potential function $F=\sum_{b \in S} u_{b}$ is of weight 1 with respect to the action. Introduce the $\mathbb{C}^{\times}$-action on Lie $\mathbb{T}$ given by the scalar multiplication; then the map $\widetilde{p r}: \mathcal{Y} \rightarrow \mathcal{M} \times$ Lie $\mathbb{T}$ is $\mathbb{C}^{\times}$-equivariant. Let $0_{ \pm}:=0_{\boldsymbol{\Sigma}_{ \pm}}=\left\{q^{ \pm \mathbf{w}}=0\right\} \in \mathcal{C}$ denote the large radius limit points of $\mathfrak{X}_{ \pm}$(see Definition 3.7) and let $\tilde{0}_{ \pm}=\tilde{0}_{\Sigma_{ \pm}} \in \operatorname{pr}^{-1}(\mathcal{C})$ denote the torus-fixed points such that $\operatorname{pr}\left(\tilde{0}_{ \pm}\right)=0_{ \pm}$ as in Section 4.3.1. The $\mathbb{C}^{\times}$-action on the family $\operatorname{pr}^{-1}(\mathcal{C}) \rightarrow \mathcal{C}$ is given by (with notation as in Lemma 5.8)

$$
s \cdot w_{v}^{ \pm}=s^{\sum_{b \in S} \Psi_{b}^{ \pm}(v)} w_{v}^{ \pm}, \quad s \cdot q^{\mathbf{w}}=s^{\sum_{b \in S} D_{b} \cdot \mathbf{w}} q^{\mathbf{w}} .
$$

By Assumption 5.2, we have that $\lim _{s \rightarrow 0} s \cdot x=0_{+}, \lim _{s \rightarrow \infty} s \cdot x=0_{-}$for every $x \in \mathcal{C} \backslash\left\{0_{+}, 0_{-}\right\}$and $\lim _{s \rightarrow 0} s \cdot y=\tilde{0}_{+}$for every $y \in \operatorname{pr}^{-1}\left(\mathcal{C} \backslash\left\{0_{-}\right\}\right)$.

We choose analytic open sets $\mathcal{B}_{ \pm} \subset \mathcal{Y}, \mathcal{U}_{ \pm} \subset \mathcal{M}_{\mathbb{T}}$ with $\tilde{0}_{ \pm} \in \mathcal{B}_{ \pm},\left(0_{ \pm}, 0\right) \in \mathcal{U}_{ \pm}$such that the conclusion of Corollary 4.20 holds. Since $\widetilde{\text { pr }}$ is $\mathbb{C}^{\times}$-equivariant, even after replacing $\mathcal{B}_{ \pm}$and $\mathcal{U}_{ \pm}$ with

$$
\bigcup_{s \in \mathbb{C}^{\times}} s \cdot \mathcal{B}_{ \pm}, \quad \text { and } \quad \bigcup_{s \in \mathbb{C}^{\times}} s \cdot \mathcal{U}_{ \pm}
$$

we have that the conclusion of Corollary 4.20 still holds. We henceforth assume that $\mathcal{B}_{ \pm}$, $\mathcal{U}_{ \pm}$are preserved by the $\mathbb{C}^{\times}$-action. Since every point in $\operatorname{pr}^{-1}\left(\mathcal{C} \backslash\left\{0_{-}\right\}\right)$flows to $\tilde{0}_{+}$under the $\mathbb{C}^{\times}$-action and $\mathcal{B}_{+}$is an open neighbourhood of $\tilde{0}_{+}$, we have that $\mathrm{pr}^{-1}\left(\mathcal{C} \backslash\left\{0_{-}\right\}\right) \subset \mathcal{B}_{+}$. Similarly we have $\left(\mathcal{C} \backslash\left\{0_{+}, 0_{-}\right\}\right) \times\{0\} \subset \mathcal{U}_{ \pm}$.

Lemma 5.11. There exists an analytic open set $\mathcal{U}_{0}$ of $\mathcal{M}_{\mathbb{T}}=\mathcal{M} \times \operatorname{Lie} \mathbb{T}$ such that

(1) $\left(\mathcal{C} \backslash\left\{0_{+}, 0_{-}\right\}\right) \times\{0\} \subset \mathcal{U}_{0} \subset \mathcal{U}_{+} \cap \mathcal{U}_{-}$;

(2) $\widetilde{\operatorname{pr}}^{-1}\left(\mathcal{U}_{0}\right) \cap \mathcal{B}_{+}=\left(\widetilde{\operatorname{pr}}^{-1}\left(\mathcal{U}_{0}\right) \cap \mathcal{B}_{-}\right) \sqcup \mathcal{R}$ for some open set $\mathcal{R}$ of $\mathcal{Y}$.

Proof. First note that $\mathcal{U}_{+} \cap \mathcal{U}_{-}$contains $\left(\mathcal{C} \backslash\left\{0_{+}, 0_{-}\right\}\right) \times\{0\}$. Since $\widetilde{\operatorname{pr}}: \mathcal{B}_{-} \rightarrow \mathcal{U}_{-}$is proper and $\mathcal{B}_{-} \backslash \mathcal{B}_{+}$is closed in $\mathcal{B}_{-}, \widetilde{\operatorname{pr}}\left(\mathcal{B}_{-} \backslash \mathcal{B}_{+}\right)$is closed in $\mathcal{U}_{-}$. Define $\mathcal{U}_{0}:=\left(\mathcal{U}_{+} \cap \mathcal{U}_{-}\right) \backslash \widetilde{\operatorname{pr}}\left(\mathcal{B}_{-} \backslash \mathcal{B}_{+}\right)$. This is an open subset of $\mathcal{U}_{+} \cap \mathcal{U}_{-}$. By definition we have $\widetilde{\mathrm{pr}}^{-1}\left(\mathcal{U}_{0}\right) \cap \mathcal{B}_{-} \subset \widetilde{\mathrm{pr}}^{-1}\left(\mathcal{U}_{0}\right) \cap \mathcal{B}_{+}$. Since we have $\widetilde{p r}^{-1}\left(\left(\mathcal{C} \backslash\left\{0_{+}, 0_{-}\right\}\right) \times\{0\}\right) \subset \operatorname{pr}^{-1}\left(\mathcal{C} \backslash\left\{0_{+}, 0_{-}\right\}\right) \subset \mathcal{B}_{+}$, we conclude $\left(\mathcal{C} \backslash\left\{0_{+}, 0_{-}\right\}\right) \times\{0\} \subset \mathcal{U}_{0}$. Finally we show that the complement of $\widetilde{\mathrm{pr}}^{-1}\left(\mathcal{U}_{0}\right) \cap \mathcal{B}_{-}$in $\widetilde{\mathrm{pr}}^{-1}\left(\mathcal{U}_{0}\right) \cap \mathcal{B}_{+}$is open. Take any point $y$ from the complement, and choose a compact neighbourhood $K$ of $\widetilde{p r}(y)$ in $\mathcal{U}_{0}$. Since $\widetilde{\mathrm{pr}}: \mathcal{B}_{-} \rightarrow \mathcal{U}_{-}$is proper, $\widetilde{\mathrm{pr}}^{-1}(K) \cap \mathcal{B}_{-}$is a compact set not containing $y$. Let $K^{\circ}$ be the interior of $K$; then $\left(\widetilde{\mathrm{pr}}^{-1}\left(K^{\circ}\right) \cap \mathcal{B}_{+}\right) \backslash\left(\widetilde{\mathrm{pr}}^{-1}(K) \cap \mathcal{B}_{-}\right)$is an open neighbourhood of $y$ which does not intersect with $\widetilde{\mathrm{pr}}^{-1}\left(\mathcal{U}_{0}\right) \cap \mathcal{B}_{-}$. 
We consider the analytified equivariant Brieskorn module $\overline{\operatorname{Bri}_{\mathbb{T}}^{\mathrm{an}}}(F)_{ \pm}:=\overline{\operatorname{Bri}_{\mathbb{T}}^{\mathrm{an}}}(F)_{\boldsymbol{\Sigma}_{ \pm}}$from Definition 4.21. Note that it is defined over the $\mathcal{U}_{ \pm}$above, since the only properties we need in the construction are those in Corollary 4.20.

Corollary 5.12. Let $\mathcal{U}_{0}, \mathcal{R}$ be as in Lemma 5.11.

(1) We have a direct sum decomposition of $\mathcal{O}_{\mathcal{U}_{0}}^{\text {an }}$-algebras:

$$
\left.\left.\left(\widetilde{\mathrm{pr}}_{*} \mathcal{O}_{\mathcal{B}_{+}}^{\mathrm{an}}\right)\right|_{\mathcal{U}_{0}} \cong\left(\widetilde{\mathrm{pr}}_{*} \mathcal{O}_{\mathcal{B}_{-}}^{\mathrm{an}}\right)\right|_{\mathcal{U}_{0}} \oplus\left(\widetilde{\mathrm{pr}}_{*} \mathcal{O}_{\mathcal{R}}^{\mathrm{an}}\right)
$$

(2) We have a direct sum decomposition of $\mathcal{O}_{\mathcal{U}_{0}}^{\text {an } \llbracket z \rrbracket-m o d u l e s: ~}$

$$
\left.\left.\overline{\operatorname{Bri}_{\mathbb{T}}^{\mathrm{an}}}(F)_{+}\right|_{\mathcal{U}_{0}} \cong \overline{\operatorname{Bri}_{\mathbb{T}}^{\mathrm{an}}}(F)_{-}\right|_{\mathcal{U}_{0}} \oplus \widetilde{\operatorname{pr}}_{*}\left(\mathcal{O}_{\mathcal{R}}^{\mathrm{an}} \llbracket z \rrbracket\right) .
$$

Under this decomposition, the Gauss-Manin connection $\nabla$, the grading operator $\mathrm{Gr}$ and the higher residue pairing $P$ split into the direct sum.

Definition 5.13. We define $\mathscr{R}:=\widetilde{\operatorname{pr}}_{*}\left(\mathcal{O}_{\mathcal{R}}^{\text {an }} \llbracket z \rrbracket\right)$. Since $\widetilde{\mathrm{pr}}: \mathcal{R} \rightarrow \mathcal{U}_{0}$ is a finite flat morphism, we can define the $\mathcal{O}_{\mathcal{U}_{0}}^{\text {an }} \llbracket z \rrbracket$-module structure, the Gauss-Manin connection $\nabla^{\mathscr{R}}$, the grading operator $\mathrm{Gr}^{\mathscr{R}}$ and the higher residue pairing $P^{\mathscr{R}}$ on $\mathscr{R}$ similarly to Definition 4.21 and Section 4.3.3. By the same argument as in Proposition $4.24, \mathscr{R}$ is a free $\mathcal{O}_{\mathcal{U}_{0}}^{\text {an }} \llbracket z \rrbracket$-module of rank $\operatorname{dim} H_{\mathrm{CR}}^{*}\left(\mathfrak{X}_{+}\right)-$ $\operatorname{dim} H_{\mathrm{CR}}^{*}\left(\mathfrak{X}_{-}\right)$. For a generic $(q, \chi) \in \mathcal{U}_{0}, \widetilde{\operatorname{pr}}^{-1}(q, \chi) \cap \mathcal{R}$ consists of finitely many reduced points, and the asymptotic expansion in Definition 4.28 defines an isomorphism Asym: $\mathscr{R}_{(q, \chi)} \cong$ $\mathbb{C} \llbracket z \rrbracket^{\oplus \operatorname{rank} \mathscr{R}}$.

Remark 5.14. Since $\operatorname{rank} \widetilde{\operatorname{pr}}_{*}\left(\mathcal{O}_{\mathcal{B}_{+}}^{\text {an }}\right)=\operatorname{dim} H_{\mathrm{CR}}^{*}\left(\mathfrak{X}_{+}\right)>\operatorname{dim} H_{\mathrm{CR}}^{*}\left(\mathfrak{X}_{-}\right)=\operatorname{rank} \widetilde{\mathrm{pr}}_{*}\left(\mathcal{O}_{\mathcal{B}_{-}}^{\text {an }}\right)($ by Corollary 4.20 and Remark 5.3), $\mathcal{R}$ is never empty under Assumption 5.2.

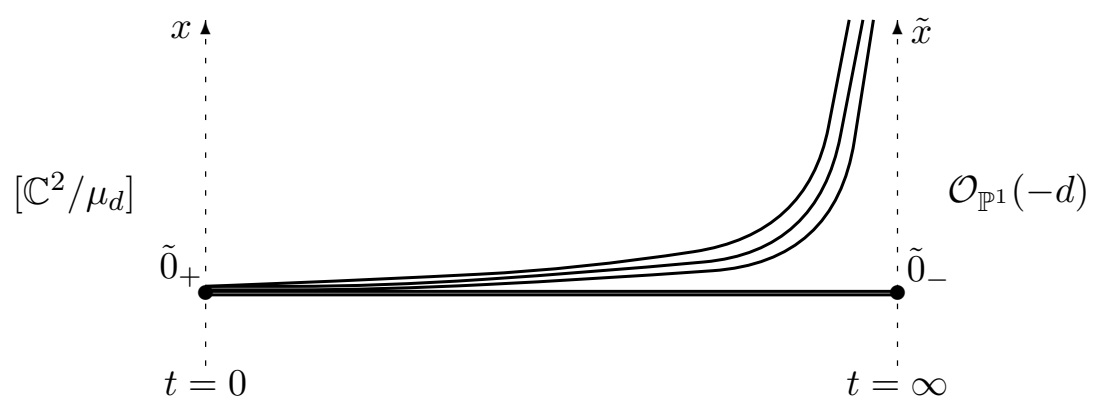

Figure 6. Family of critical points. Out of $d$ critical points, $d-2$ points go to infinity at the large radius limit of $\mathcal{O}_{\mathbb{P}^{1}}(-d)(d=5$ in the picture $)$.

Example 5.15. Consider the example of the LG model in Section 3.6.3. This corresponds to a discrepant transformation between $\mathfrak{X}_{+}=\left[\mathbb{C}^{2} / \mu_{d}\right]$ (of type $\frac{1}{d}(1,1)$ ) and its minimal resolution $\mathfrak{X}_{-}=\mathcal{O}_{\mathbb{P}^{1}}(-d)$. The LG model was given by the potential function

$$
F=x_{2}+\frac{x_{1}^{d}}{x_{2}}+t x_{1}=\tilde{x}_{2}+q \frac{\tilde{x}_{1}^{d}}{\tilde{x}_{2}}+\tilde{x}_{1},
$$

where $\left(x_{1}, x_{2}, t\right)$ and $\left(\tilde{x}_{1}, \tilde{x}_{2}, q\right)$ are related by $\tilde{x}_{1}=t x_{1}, \tilde{x}_{2}=x_{2}, q=t^{-d}$. Here $q=t^{-d}$ is a co-ordinate on $\mathcal{M}=\mathbb{P}(1, d),\{t=0\}$ is the large radius limit point of $\mathfrak{X}_{+}$and $\{q=0\}$ is the large radius limit point of $\mathfrak{X}_{-}$. On the chart near $t=0$ (associated to $\mathfrak{X}_{+}$), the precise domain of definition of the potential function $F$ is given by (see Section 3.6.3)

$$
\mathcal{Y}_{\Sigma_{+}}=\left\{\left(x_{1}, x_{2}, y, t\right) \in \mathbb{C}^{4}: x_{2} y=x_{1}^{d}\right\},
$$


where $y$ is identified with the term $x_{1}^{d} / x_{2}$ in $F$. The relative critical scheme of $F$ on this chart is given by

$$
\begin{aligned}
\left\{x_{1} \frac{\partial F}{\partial x_{1}}=x_{2} \frac{\partial F}{\partial x_{2}}=0\right\} & =\left\{d \frac{x_{1}^{d}}{x_{2}}+t x_{1}=0, x_{2}-\frac{x_{1}^{d}}{x_{2}}=0\right\} \\
& =\left\{\left(x_{1}, x_{2}, y, t\right) \in \mathbb{C}^{4}: d y+t x_{1}=0, x_{2}-y=0, x_{2} y=x_{1}^{d}\right\} \\
& =\left\{\left(x_{1},-\frac{t x_{1}}{d},-\frac{t x_{1}}{d}, t\right) \in \mathbb{C}^{4}: x_{1}^{2}\left(x_{1}^{d-2}-t^{2} / d^{2}\right)=0\right\} .
\end{aligned}
$$

This is schematically depicted in Fig. 6.

\subsection{Comparison of quantum D-modules}

Finally we obtain a comparison result between the quantum D-modules of $\mathfrak{X}_{+}$and $\mathfrak{X}_{-}$combining Theorem 4.34 and Corollary 5.12.

Recall the analytic quantum D-module $\mathrm{QDM}_{\mathbb{T}}^{\mathrm{an}}\left(\mathfrak{X}_{ \pm}\right)$of $\mathfrak{X}_{ \pm}$from Section 4.4; it is defined over an open neighbourhood $U_{ \pm}$of the origin in the equivariant Kähler moduli space

$$
\left[\mathcal{M}_{\mathrm{A}, \mathbb{T}}\left(\mathfrak{X}_{ \pm}\right) / \operatorname{Pic}^{\mathrm{st}}\left(\mathfrak{X}_{ \pm}\right)\right] \text {. }
$$

By Theorem 4.34, we have the analytic mirror isomorphism

$$
\operatorname{Mir}_{ \pm}^{\text {an }}:\left.\overline{\operatorname{Bri}_{\mathbb{T}}^{\mathrm{an}}}(F)_{ \pm}\right|_{\mathcal{U}_{ \pm}^{\prime}} \cong \operatorname{mir}_{ \pm}^{*} \overline{\operatorname{QDM}_{\mathbb{T}}^{\mathrm{an}}}\left(\mathfrak{X}_{ \pm}\right)
$$

over an open neighbourhood $\mathcal{U}_{ \pm}^{\prime} \subset \mathcal{U}_{ \pm}$of $\left(0_{ \pm}, 0\right) \in \mathcal{M}_{\mathbb{T}}=\mathcal{M} \times$ Lie $\mathbb{T}$, where $\operatorname{mir}_{ \pm}: \mathcal{U}_{ \pm}^{\prime} \rightarrow U_{ \pm}$ denotes the mirror map and the overline $\cdots$ means the $z$-adic completion. Let us observe that the analytic mirror isomorphism extends to a domain which is closed under the $\mathbb{C}^{\times}$action (as discussed in Section 5.3). We have already seen in Section 5.3 that $\overline{\operatorname{Bri}} i_{\mathbb{T}}^{\text {an }}(F)_{ \pm}$ extends to $\mathbb{C}^{\times} \mathcal{U}_{ \pm}^{\prime}=\bigcup_{s \in \mathbb{C}^{\times}} s \cdot \mathcal{U}_{ \pm}^{\prime}$. Introduce the $\mathbb{C}^{\times}$-action on the equivariant Kähler moduli space $\left[\mathcal{M}_{\mathrm{A}, \mathbb{T}}\left(\mathfrak{X}_{ \pm}\right) / \mathrm{Pic}^{\text {st }}\left(\mathfrak{X}_{ \pm}\right)\right]$generated by the Euler vector field $(2.7)$; since the mirror map mir $_{ \pm}$preserves the Euler vector fields, it can be extended to a unique $\mathbb{C}^{\times}$-equivariant map $\operatorname{mir}_{ \pm}: \mathbb{C}^{\times} \mathcal{U}_{ \pm}^{\prime} \rightarrow \mathbb{C}^{\times} U_{ \pm}$. We may assume (by shrinking $\mathcal{U}^{\prime}$ if necessary) that $\left.\overline{\operatorname{Bri}} i_{\mathbb{T}}^{\text {an }}(F)_{ \pm}\right|_{\mathcal{U}^{\prime}}$ is generated by Gr-homogeneous sections $\Omega_{0}^{ \pm}, \ldots, \Omega_{s}^{ \pm}$over $\mathcal{O}_{\mathcal{U}_{ \pm}^{\prime}}^{\text {an }} \llbracket z \rrbracket$ (see [27, Theorem 4.26] and Part (b) in Section 4.4). Since Mir ${ }^{\text {an }}$ intertwines the grading operators as $\mathrm{Mir}^{\mathrm{an}} \circ \mathrm{Gr}=\mathrm{Gr} \circ \mathrm{Mir}^{\mathrm{an}}$, the sections $\operatorname{Mir}^{\text {an }}\left(\Omega_{i}^{ \pm}\right)$extend to $\mathbb{C}^{\times} \mathcal{U}_{ \pm}^{\prime}$, and the mirror isomorphism $\operatorname{Mir}^{\text {an }}$ also extends there.

Henceforth we assume that the open set $\mathcal{U}_{ \pm}^{\prime}$ (where the analytic mirror isomorphism (5.4) is defined) is closed under the $\mathbb{C}^{\times}$-action. By the same argument as in Section 5.3, $\mathcal{U}_{+}^{\prime} \cap \mathcal{U}_{-}^{\prime}$ is an open set containing $\left(\mathcal{C} \backslash\left\{0_{+}, 0_{-}\right\}\right) \times\{0\} \subset \mathcal{M}_{\mathbb{T}}$. Setting $\mathcal{U}_{0}^{\prime}=\mathcal{U}_{0} \cap\left(\mathcal{U}_{+}^{\prime} \cap \mathcal{U}_{-}^{\prime}\right)$ for the open set $\mathcal{U}_{0}$ from Lemma 5.11, we obtain the following result.

Theorem 5.16. There exist an open subset $\mathcal{U}_{0}^{\prime}$ of $\mathcal{M}_{\mathbb{T}}$ containing $\left(\mathcal{C} \backslash\left\{0_{+}, 0_{-}\right\}\right) \times\{0\}$ and mirror maps $\operatorname{mir}_{ \pm}: \mathcal{U}_{0}^{\prime} \rightarrow\left[\mathcal{M}_{\mathrm{A}, \mathbb{T}}\left(\mathfrak{X}_{ \pm}\right) / \mathrm{Pic}^{\text {st }}\left(\mathfrak{X}_{ \pm}\right)\right]$to the equivariant Kähler moduli spaces of $\mathfrak{X}_{ \pm}$such that the following decomposition of $\mathcal{O}_{\mathcal{U}_{0}^{\prime}}^{\text {an }} \llbracket z \rrbracket$-modules holds:

$$
\left.\operatorname{mir}_{+}^{*} \overline{\mathrm{QDM}_{\mathbb{T}}^{\mathrm{an}}}\left(\mathfrak{X}_{+}\right) \cong \operatorname{mir}_{-}^{*} \overline{\mathrm{QDM}_{\mathbb{T}}^{\mathrm{an}}}\left(\mathfrak{X}_{-}\right) \oplus \mathscr{R}\right|_{\mathcal{U}_{0}^{\prime}},
$$

where $\overline{\mathrm{QDM}_{\mathbb{T}}^{\mathrm{an}}}\left(\mathfrak{X}_{ \pm}\right)$denotes the z-adic completion of the equivariant quantum D-module of $\mathfrak{X}_{ \pm}$ (see Section 4.4) and $\mathscr{R}$ is as in Definition 5.13. Under this decomposition, the flat connection, the grading operator and the pairing split as follows:

(1) $\operatorname{mir}_{+}^{*} \nabla^{+}=\left(\right.$mir $\left._{-}^{*} \nabla^{-}+\alpha \cdot \mathrm{id}\right) \oplus \nabla^{\mathscr{R}}$ for some 1 -form $\alpha \in \Omega_{\mathcal{M}_{\mathbb{T}} / \text { Lie } \mathbb{T}}^{1}$; 
(2) $\operatorname{mir}_{+}^{*} \mathrm{Gr}^{+}=\operatorname{mir}_{-}^{*} \mathrm{Gr}^{-} \oplus \mathrm{Gr}^{\mathscr{R}}$;

(3) $\operatorname{mir}_{+}^{*} P^{+}=\operatorname{mir}_{-}^{*} P^{-} \oplus P^{\mathscr{R}}$,

where $\nabla^{ \pm}, \mathrm{Gr}^{ \pm}, P^{ \pm}$denote respectively the quantum connection, the grading operator (2.8) and the pairing (2.9) on the quantum D-module of $\mathfrak{X}_{ \pm}$.

Remark 5.17. The one-form $\alpha$ arises from the difference of the splittings of the extended divisor sequence (3.4) over $\mathbb{Q}$, one chosen for $\mathfrak{X}_{+}$and the other for $\mathfrak{X}_{-}$(see Remark 4.35). It is of the form $\alpha\left(\xi q \frac{\partial}{\partial q}\right)=\bar{\alpha}(\xi)$ for some $\bar{\alpha} \in \operatorname{Hom}\left(\mathbb{L}_{\mathbb{Q}}^{\star}, \mathbf{M}_{\mathbb{Q}}^{\star}\right)$; in particular $\alpha$ vanishes in the non-equivariant limit. Recall that the Gauss-Manin connection in the equivariant case depends on the choice of a splitting (see Section 4.1).

Remark 5.18. By construction, the above decomposition (5.5) preserves the additional structure given by multiplication by the mirror co-ordinates $x_{1}, \ldots, x_{n}$. They correspond to the equivariant shift operators (Seidel representation), see Remark 4.3.

\subsection{Comparison of Gromov-Witten theories in all genera}

Using a formula due to Givental $[49,50]$ and Teleman [104] for higher genus Gromov-Witten potentials, we show that the ancestor Gromov-Witten potential of $\mathfrak{X}_{+}$is decomposed into the ancestor potential of $\mathfrak{X}_{-}$and a product of Witten-Kontsevich tau functions, under the action of a quantized symplectic operator. We use Givental's formula for orbifolds studied by BriniCavalieri-Ross [19] and Zong [109].

\subsubsection{Ancestor potentials}

Let $\mathfrak{X}$ be a smooth DM stack equipped with a $\mathbb{T}$-action satisfying the assumptions in Section 2.1; we use the notation there. Consider the forgetful morphism $p: \mathfrak{X}_{g, l+m, d} \rightarrow \bar{M}_{g, l+m} \rightarrow \bar{M}_{g, l}$ which forgets the map and the last $m$ marked points, and let $\bar{\psi}_{i} \in H^{2}\left(\mathfrak{X}_{g, l+m, d}\right), 1 \leq i \leq l$ denote the pull-back of the universal cotangent class $\psi_{i} \in H^{2}\left(\bar{M}_{g, l}\right)$ by $p$. We define the ancestor Gromov-Witten invariants as the $\mathbb{T}$-equivariant integral

$$
\left\langle\alpha_{1} \bar{\psi}^{k_{1}}, \ldots, \alpha_{l} \bar{\psi}^{k_{l}} ; \beta_{1}, \ldots, \beta_{m}\right\rangle_{g, l+m, d}:=\int_{\left[\mathfrak{X}_{g, l+m, d}\right]_{\mathrm{vir}}} \prod_{i=1}^{l} \mathrm{ev}_{i}^{*}\left(\alpha_{i}\right) \bar{\psi}_{i}^{k_{i}} \cdot \prod_{i=1}^{m} \mathrm{ev}_{i+l}^{*}\left(\beta_{i}\right),
$$

where $\alpha_{i}, \beta_{j} \in H_{\mathrm{CR}, \mathbb{T}}^{*}(\mathfrak{X})$. When the moduli stack $\mathfrak{X}_{g, m+l, d}$ is not proper, the right-hand side is defined by the virtual localization formula and lies in $S_{\mathbb{T}}=\operatorname{Frac}\left(R_{\mathbb{T}}\right)$ as before. We choose a homogeneous $R_{\mathbb{T}}$-basis $\left\{\phi_{i}\right\}_{i=0}^{N}$ of $H_{\mathrm{CR}, \mathbb{T}}^{*}(\mathfrak{X})$ satisfying $(2.4)$ and introduce the infinite set $\mathbf{y}=\left\{y_{k}^{i}\right\}_{k \geq 0,0 \leq i \leq s}$ of co-ordinates on $H_{\mathrm{CR}, \mathbb{T}}^{*}(\mathfrak{X}) \llbracket z \rrbracket$ given by

$$
\mathbf{y} \mapsto \mathbf{y}(z)=\sum_{k=0}^{\infty} \sum_{i=0}^{s} y_{k}^{i} \phi_{i} z^{k} .
$$

The ancestor potential of $\mathfrak{X}$ is the following generating function of the ancestor Gromov-Witten invariants

$$
\mathscr{A}_{\mathfrak{X}, \tau}=\exp \left(\sum_{d \in \boldsymbol{\Lambda}_{+}} \sum_{\substack{g \geq 0, l \geq 0 \\ 2 g-2+l>0}} \sum_{m=0}^{\infty}\langle\mathbf{y}(\bar{\psi}), \ldots, \mathbf{y}(\bar{\psi}) ; \tau, \ldots, \tau\rangle_{g, l+m, d} \hbar^{g-1} \frac{Q^{d}}{l ! m !}\right),
$$

which we regard as a function in the formal neighbourhood of $\mathbf{y}=0$. Introduce another set of variables $\mathbf{x}=\left\{x_{k}^{i}\right\}_{k \geq 0,0 \leq i \leq N}$ that are related to $\mathbf{y}$ by the formula (Dilaton shift)

$$
\mathbf{x}(z)=\mathbf{y}(z)-\phi_{0} z
$$


where $\mathbf{x}(z)=\sum_{k=0}^{\infty} \sum_{i=0}^{N} x_{k}^{i} \phi_{i} z^{k}$. Using the co-ordinate $\mathbf{x}(z)$, we shall regard $\mathscr{A}_{\mathfrak{X}, \tau}$ as a formal function on the formal neighbourhood of $\mathbf{x}(z)=-\phi_{0} z$ in $H_{\mathrm{CR}, \mathbb{T}}^{*}(\mathfrak{X}) \llbracket z \rrbracket$.

As in Section 2.2, we can specialize $Q$ to one in the ancestor potential by using the divisor equation for $\tau$. We have

$$
\mathscr{A}_{\mathfrak{X}, \tau}=\exp \left(\sum_{d \in \boldsymbol{\Lambda}_{+}} \sum_{\substack{g \geq 0, l \geq 0 \\ 2 g-2+l>0}} \sum_{m=0}^{\infty}\left\langle\mathbf{y}(\bar{\psi}), \ldots, \mathbf{y}(\bar{\psi}) ; \tau^{\prime}, \ldots, \tau^{\prime}\right\rangle_{g, l+m, d} \hbar^{g-1} \frac{e^{\sigma \cdot d} Q^{d}}{l ! m !}\right),
$$

when $\tau=\sigma+\tau^{\prime}$ with $\sigma=\sum_{i=1}^{r} \tau^{i} \phi_{i} \in H^{2}(\mathfrak{X})$ and $\tau^{\prime}=\tau^{0} \phi_{0}+\sum_{i=r+1}^{s} \tau^{i} \phi_{i}$. Moreover, the Galois symmetry (2.3) implies

$$
\mathscr{A}_{\mathfrak{X}, g(\xi) \tau}(d g(\xi) \mathbf{x})=\mathscr{A}_{\mathfrak{X}, \tau}(\mathbf{x}), \quad \xi \in H^{2}(\mathfrak{X}, \mathbb{Z}),
$$

where $g(\xi), d g(\xi)$ are as in Section 2.2 (they act on the equivariant cohomology $H_{\mathrm{CR}, \mathbb{T}}^{*}(\mathfrak{X})$ via the splitting $H_{\mathrm{CR}, \mathbb{T}}^{*}(\mathfrak{X}) \cong H_{\mathrm{CR}}^{*}(\mathfrak{X}) \otimes R_{\mathbb{T}}$ given by the basis $\left.\left\{\phi_{i}\right\}\right)$. Therefore the specialization $\left.\mathscr{A}_{\mathfrak{X}, \tau}\right|_{Q=1}$ makes sense as an element of $S_{\mathbb{T}}((\hbar)) \llbracket \boldsymbol{\Lambda}_{+} \rrbracket \llbracket \tau^{\prime}, \mathbf{y} \rrbracket-$ we regard it as an $\left(H^{2}(\mathfrak{X}, \mathbb{Z}) / \boldsymbol{\Lambda}^{\star}\right)$ invariant function of $(\tau, \mathbf{x}) \in \mathcal{M}_{\mathrm{A}, \mathbb{T}}(\mathfrak{X}) \times_{\operatorname{Spec} R_{\mathbb{T}}} \mathbf{H}_{\mathrm{CR}, \mathbb{T}}(\mathfrak{X}) \llbracket z \rrbracket$, where $\mathbf{H}_{\mathrm{CR}, \mathbb{T}}(\mathfrak{X}) \llbracket z \rrbracket$ denotes the infinite-dimensional vector bundle over $\operatorname{Spec} R_{\mathbb{T}}$ associated with the $R_{\mathbb{T}}$-module $H_{\mathrm{CR}, \mathbb{T}}^{*}(\mathfrak{X}) \llbracket z \rrbracket$ (see Remark 2.2). Henceforth we assume that $Q$ is specialized to one in the ancestor potential.

\subsubsection{Givental's quantization formalism}

We briefly review the quantization formalism of Givental [49]. We also refer to [29, Section 3], [30, Section 5.1] for the exposition.

Let $V$ be a finite-dimensional $\mathbb{C}$-vector space equipped with a symmetric non-degenerate pairing $(\cdot, \cdot)_{V}$. Then $V((z))$ has the following symplectic form $\Omega_{V}$ :

$$
\Omega_{V}(f, g)=\operatorname{Res}_{z=0}(f(-z), g(z))_{V} d z .
$$

Choosing a basis $\left\{\phi_{i}\right\}$ on $V$, we let $\mathbf{x}=\left\{x_{k}^{i}\right\}_{k \geq 0,0 \leq i \leq s}$ denote the co-ordinates on $V \llbracket z \rrbracket$ given by $\mathbf{x} \mapsto \mathbf{x}(z)=\sum_{k=0}^{\infty} \sum_{i=0}^{s} x_{k}^{i} \phi_{i} z^{k}$

Let $W$ be another $\mathbb{C}$-vector space of the same dimension equipped with a symmetric nondegenerate pairing $(\cdot, \cdot)_{W}$. A $\mathbb{C} \llbracket z \rrbracket$-linear operator $U: V \llbracket z \rrbracket \rightarrow W \llbracket z \rrbracket$ is said to be unitary when it satisfies

$$
\left(U(-z) v_{1}, U(z) v_{2}\right)_{W}=\left(v_{1}, v_{2}\right)_{V} .
$$

A unitary operator $U$ induces a $\mathbb{C}((z))$-linear symplectic transformation $U: V((z)) \rightarrow W((z))$. The quantized operator $\widehat{U}$ described below sends a certain "tame" (spelled out below) formal function $\mathscr{A}$ on $V \llbracket z \rrbracket$ to a tame formal function $\widehat{U} \mathscr{A}$ on $W \llbracket z \rrbracket$. For $\mathbf{D} \in z V \llbracket z \rrbracket$, let $\mathfrak{A} \mathfrak{F o c k}(V, \mathbf{D})$ denote the set of formal power series $\mathscr{A}$ of the form

$$
\mathscr{A}=\exp \left(\sum_{g=0}^{\infty} \hbar^{g-1} F^{g}(\mathbf{x})\right)
$$

with $F^{g}$ a formal power series in $\mathbf{x}-\mathbf{D}$ (i.e., a function in the formal neighbourhood of $\mathbf{x}=\mathbf{D}$ in $V \llbracket z \rrbracket)$ satisfying the following tameness condition

$$
\left.\frac{\partial^{m} F^{g}}{\partial x_{k_{1}}^{i_{1}} \cdots \partial x_{k_{m}}^{i_{m}}}\right|_{\mathbf{x}=\mathbf{D}}=0 \quad \text { if } k_{1}+\cdots+k_{m}>3 g-3+m
$$


and $\left.F^{0}\right|_{\mathbf{x}=\mathbf{D}}=\left.F^{1}\right|_{\mathbf{x}=\mathbf{D}}=0$ (see [30, Definition 5.1]). Then $\widehat{U}$ defines an operator [30, Definition 5.7]:

$$
\widehat{U}: \quad \mathfrak{A} \mathfrak{F o c k}(V, \mathbf{D}) \rightarrow \mathfrak{A} \mathfrak{F o c k}(W, U(\mathbf{D})) .
$$

For $\mathbf{s} \in z V \llbracket z \rrbracket$, let $T_{\mathbf{s}}$ denote the operator acting on tame functions on $V \llbracket z \rrbracket$ which shifts the base point $\mathbf{D}$ by $\mathbf{s}$ [30, Definition 5.5]; for a tame function $\mathscr{A} \in \mathfrak{A} \mathfrak{F o c k}(V, \mathbf{D}), T_{\mathbf{s}} \mathscr{A} \in \mathfrak{A F} \mathfrak{F c k}(V, \mathbf{D}+\mathbf{s})$ denotes the Taylor expansion of $e^{-F_{1}(\mathbf{D}+\mathbf{s})} \mathscr{A}$ at the new base point $\mathbf{D}+\mathbf{s}$ (whenever it makes sense). The tameness condition implies that $T_{\mathbf{s}} \mathscr{A}$ is well-defined for all $\mathscr{A} \in \mathfrak{A} \mathfrak{F o c k}(V, \mathbf{D})$ if $\mathbf{s} \in z^{2} V \llbracket z \rrbracket$; under the additional assumption that $\mathscr{A}$ is rational [30, Definition 5.2], $T_{\mathbf{s}} \mathscr{A}$ is well-defined for $\mathbf{s} \in z V \llbracket z \rrbracket[30$, Definition 5.5]. All tame functions appearing in this section are rational, since they are obtained by the Givental formula [29, Proposition 3.20].

When the ancestor potential $\mathscr{A}_{\mathfrak{X}, \tau}$ is convergent and analytic in the variable $\tau$, it defines an element of $\mathfrak{A} \mathfrak{F} \mathfrak{o c k}\left(\mathbf{H}_{\mathrm{CR}, \mathbb{T}}(\mathfrak{X})_{\chi},-z \phi_{0}\right)$ where $\mathbf{H}_{\mathrm{CR}, \mathbb{T}}(\mathfrak{X})_{\chi}$ denotes the fibre of the vector bundle $\mathbf{H}_{\mathrm{CR}, \mathbb{T}}(\mathfrak{X}) \rightarrow \operatorname{Spec} R_{\mathbb{T}}$ (see Remark 2.2) at $\chi$ and $\chi$ is the image of $\tau \in \mathcal{M}_{\mathrm{A}, \mathbb{T}}(\mathfrak{X})$ in Spec $R_{\mathbb{T}}$.

The Witten-Kontsevich tau-function is the ancestor potential for a point. It is a function on the formal neighbourhood of $-z$ in $\mathbb{C} \llbracket z \rrbracket$ given by

$$
\begin{aligned}
\mathscr{T} & =\exp \left(\sum_{g=0}^{\infty} \hbar^{g-1} \sum_{l: 2 g-2+l>0} \frac{1}{l !} \sum_{k_{1}, \ldots, k_{l} \geq 0}\left\langle\psi^{k_{1}}, \ldots, \psi^{k_{l}}\right\rangle_{g, l} y_{k_{1}} \cdots y_{k_{l}}\right) \\
& =\left(-x_{1}\right)^{-\frac{1}{24}} \exp \left(\sum_{g=0}^{\infty} \hbar^{g-1} \sum_{l: 2 g-2+l>0} \frac{1}{l !} \sum_{\substack{k_{1}, \ldots, k_{l} \geq 0 \\
k_{j} \neq 1}}\left\langle\psi^{k_{1}}, \cdots, \psi^{k_{l}}\right\rangle_{g, l} \frac{x_{k_{1}} \cdots x_{k_{l}}}{\left(-x_{1}\right)^{2 g-2+l}}\right),
\end{aligned}
$$

where

$$
\left\langle\psi^{k_{1}}, \ldots, \psi^{k_{l}}\right\rangle_{g, l}=\int_{\bar{M}_{g, l}} \psi^{k_{1}} \cdots \psi^{k_{l}}
$$

$\sum_{k=0}^{\infty} x_{k} z^{k}$ denotes the co-ordinate on $\mathbb{C} \llbracket z \rrbracket$ and $y_{k}=x_{k}+\delta_{k, 1}$. We used the dilaton equation [2, Theorem 8.3.1] in the second line. The Witten-Kontsevich tau-function defines a rational element of $\mathfrak{A} \mathfrak{F} \mathfrak{o c k}(\mathbb{C},-z)$.

\subsubsection{A relationship between ancestor potentials}

We fix a homogeneous $R_{\mathbb{T}}$-basis $\left\{\phi_{i}\right\}$ of $H_{\mathrm{CR}, \mathbb{T}}^{*}\left(\mathfrak{X}_{ \pm}\right)$satisfying $(2.4)$ as above. Let $\mathcal{U}_{0}^{\prime} \subset \mathcal{M}_{\mathbb{T}}$ be an open set from Theorem 5.16. As discussed in Section 4.3.3, there exists an open dense subset $\mathcal{U}_{0}^{\prime \text { ss }}$ of $\mathcal{U}_{0}^{\prime}$ such that for each $(q, \chi) \in \mathcal{U}_{0}^{\prime \text { ss }}, \widetilde{\mathrm{pr}}^{-1}(q, \chi) \cap \mathcal{B}_{+}$consists of $\operatorname{dim} H_{\mathrm{CR}}^{*}\left(\mathfrak{X}_{+}\right)$ many reduced points. Choose a point $(q, \chi) \in \mathcal{U}_{0}^{\prime \text { ss }}$. Combining the decomposition (5.5) at $(q, \chi)$ and the isomorphism Asym: $\mathscr{R}_{(q, \chi)} \cong \mathbb{C} \llbracket z \rrbracket^{\oplus \operatorname{rank} \mathscr{R}}$ given by the asymptotic expansion (see Definition 5.13), we obtain a $\mathbb{C} \llbracket z \rrbracket$-linear isomorphism

$$
\widetilde{U}_{q, \chi}: \mathbf{H}_{\mathrm{CR}, \mathbb{T}}\left(\mathfrak{X}_{+}\right)_{\chi} \llbracket z \rrbracket \stackrel{\cong}{\rightrightarrows} \mathbf{H}_{\mathrm{CR}, \mathbb{T}}\left(\mathfrak{X}_{-}\right)_{\chi} \llbracket z \rrbracket \oplus \mathbb{C} \llbracket z \rrbracket^{\oplus \operatorname{rank} \mathscr{R}},
$$

where $\mathbf{H}_{\mathrm{CR}, \mathbb{T}}\left(\mathfrak{X}_{ \pm}\right)_{\chi}$ denotes the fibre of the vector bundle $\mathbf{H}_{\mathrm{CR}, \mathbb{T}}\left(\mathfrak{X}_{ \pm}\right) \rightarrow$ Spec $R_{\mathbb{T}}$ at $\chi$ (see Remark 2.2). The equivariant orbifold Poincaré pairing (2.2) defines a $\mathbb{C}$-bilinear pairing on $\mathbf{H}_{\mathrm{CR}, \mathbb{T}}\left(\mathfrak{X}_{ \pm}\right)_{\chi}$ for a generic $\chi$. With respect to these pairings and the diagonal pairing on $\mathbb{C} \llbracket z \rrbracket^{\oplus \operatorname{rank} \mathscr{R}}, U=\widetilde{U}_{q, \chi}$ satisfies the unitarity (5.6); this follows from Theorem 5.16 and the definition of the higher residue pairing (see Section 4.3.3). We flip the sign of $z$ and set $U_{q, \chi}:=\left.\widetilde{U}_{q, \chi}\right|_{z \rightarrow-z}$. 
When functions $\mathscr{A}_{i}$ on $V_{i} \llbracket z \rrbracket(i=1, \ldots, k)$ are given, we write $\mathscr{A}_{1} \otimes \cdots \otimes \mathscr{A}_{k}$ for the function on $V_{1} \llbracket z \rrbracket \times \cdots \times V_{k} \llbracket z \rrbracket$ given by $\left(\mathbf{x}^{(1)}, \ldots, \mathbf{x}^{(k)}\right) \mapsto \prod_{i=1}^{k} \mathscr{A}_{i}\left(\mathbf{x}^{(i)}\right)$.

Theorem 5.19. Let $\mathscr{A}_{ \pm, \tau}$ denote the ancestor potential of $\mathfrak{X}_{ \pm}$. For $(q, \chi) \in \mathcal{U}_{0}^{\text {ss }}$, we have

$$
T_{\mathbf{s}} \widehat{U}_{q, \chi} \mathscr{A}_{+, \operatorname{mir}_{+}(q, \chi)}=\mathscr{A}_{-, \operatorname{mir}_{-}(q, \chi)} \otimes \mathscr{T}^{\otimes \operatorname{rank} \mathscr{R}},
$$

where $\mathbf{s}:=\left(-z \phi_{0},(-z, \ldots,-z)\right)+U_{q, \chi}\left(z \phi_{0}\right) \in \mathbf{H}_{\mathrm{CR}, \mathbb{T}}\left(\mathfrak{X}_{-}\right)_{\chi} \llbracket z \rrbracket \times \mathbb{C} \llbracket z \rrbracket^{\oplus \operatorname{rank} \mathscr{R}}$.

Remark 5.20. Implicit in the above theorem is the convergence and analyticity of $\mathscr{A}_{ \pm, \tau}$ with respect to $\tau$. This follows from the Givental formula. We refer to [29, Theorem 1.4, Definition 3.13] for the discussion on the convergence of ancestor potentials.

Remark 5.21. The ancestor potentials of $\mathfrak{X}_{ \pm}$define sections of the Fock sheaves [30] associated with the quantum $\mathrm{D}$-modules of $\mathfrak{X}_{ \pm}$, and the above relationship can be interpreted in this language.

Remark 5.22. We can state the relationship between the Gromov-Witten theories of $\mathfrak{X}_{+}$ and $\mathfrak{X}_{-}$in terms of cohomological field theory (CohFT): the CohFT of $\mathfrak{X}_{+}$transforms into the product of the CohFT of $\mathfrak{X}_{-}$and the trivial semisimple CohFT under the Givental action of $U_{q, \chi}$. We refer the reader to $[88,102,104]$ for the Givental group action on CohFTs.

\subsubsection{Givental's formula and the proof of Theorem 5.19}

Teleman's classification theorem [104] implies that the ancestor potential $\mathscr{A}$ of a semisimple cohomological field theory on a vector space $H$ lies in the orbit of $\mathscr{T}^{\otimes N}(N=\operatorname{dim} H)$ with respect to the action of symplectic operators (via the Givental quantization). That is, there exists a unitary operator $R: \mathbb{C} \llbracket z \rrbracket^{\oplus N} \rightarrow H \llbracket z \rrbracket$ such that

$$
\mathscr{A}=T_{-z e+R(z, \ldots, z)} \widehat{R} \mathscr{T}^{\otimes N},
$$

where $e \in H$ is the identity element of the Frobenius algebra $H$. In our context, $R$ depends on $\tau \in \mathbf{H}_{\mathrm{CR}, \mathbb{T}}(\mathfrak{X})$ and gives a formal fundamental solution of the quantum connection in the $\tau$-direction. It is ambiguous up to the right multiplication by a constant diagonal matrix [48, Remarks after Proposition 1.1]; as [19, 109] did, the ambiguity can be fixed by Tseng's orbifold quantum Riemann-Roch theorem [105].

We state Givental's formula for a semiprojective toric DM stack in terms of the mirror LG model. Let $\mathfrak{X}_{\boldsymbol{\Sigma}}$ be a smooth toric DM stack with $\boldsymbol{\Sigma} \in \mathfrak{F} \mathfrak{a n}(S)$ and let $(\operatorname{pr}: \mathcal{Y} \rightarrow \mathcal{M}, F$ ) denote the mirror LG model from Definition 3.6. Let $\mathcal{B} \subset \mathcal{Y}, \mathcal{U} \subset \mathcal{M}_{\mathbb{T}}:=\mathcal{M} \times$ Lie $\mathbb{T}$ be analytic open neighbourhoods of $\tilde{0}_{\boldsymbol{\Sigma}}$ and $\left(0_{\boldsymbol{\Sigma}}, 0\right)$ respectively such that the conclusion of Corollary 4.20 holds, as usual. Let $\mathcal{U}^{\text {ss }} \subset \mathcal{U}$ denote the open dense subset consisting of $(q, \chi) \in \mathcal{U}$ such that $\widetilde{p r}^{-1}(q, \chi) \cap \mathcal{B}$ is reduced (recall, as discussed in Section 4.3.3, that $\widetilde{\mathrm{pr}}^{-1}(q, \chi)$ consists of relative critical points of the equivariant potential $\left.F_{\mathbb{T}}=F-\sum_{i=1}^{n} \chi_{i} \log x_{i}\right)$. For $(q, \chi) \in \mathcal{U}^{\text {ss }}$, the asymptotic expansion of equivariant oscillatory integrals (see Definition 4.28) defines an isomorphism

$$
\text { Asym: }\left.\overline{\operatorname{Bri}_{\mathbb{T}}^{\text {an }}}(F)_{\Sigma}\right|_{(q, \chi)} \rightarrow \mathbb{C} \llbracket z \rrbracket^{\oplus N}, \quad \text { Asym }:=\bigoplus_{p \in \widetilde{\operatorname{pr}}^{-1}(q, \chi) \cap \mathcal{B}} \operatorname{Asym}_{p},
$$

where $N=\operatorname{deg}\left(\left.\widetilde{\operatorname{pr}}\right|_{\mathcal{B}}\right)=\operatorname{dim} H_{\mathrm{CR}}^{*}(\mathfrak{X})$. Composing the inverse map with the mirror isomorphism from Theorem 4.34, we obtain a linear map

$$
\widetilde{R}_{q, \chi}:\left.\left.\quad \mathbb{C} \llbracket z \rrbracket^{\oplus N} \stackrel{\mathrm{Asym}^{-1}}{\longrightarrow} \overline{\operatorname{Bri}_{\mathbb{T}}^{\text {an }}}(F)_{\Sigma}\right|_{(q, \chi)} \stackrel{\operatorname{Mir}^{\text {an }}}{\longrightarrow} \overline{\operatorname{QDM}_{\mathbb{T}}^{\text {an }}}\left(\mathfrak{X}_{\boldsymbol{\Sigma}}\right)\right|_{\operatorname{mir}(q, \chi)} \cong \mathbf{H}_{\mathrm{CR}, \mathbb{T}}\left(\mathfrak{X}_{\boldsymbol{\Sigma}}\right)_{\chi} \llbracket z \rrbracket,
$$


where mir $: \mathcal{U} \rightarrow\left[\mathcal{M}_{\mathrm{A}, \mathbb{T}}\left(\mathfrak{X}_{\boldsymbol{\Sigma}}\right) / \mathrm{Pic}^{\mathrm{st}}\left(\mathfrak{X}_{\boldsymbol{\Sigma}}\right)\right]$ is the mirror map. We flip the sign of $z$ and set $R_{q, \chi}:=\left.\widetilde{R}_{q, \chi}\right|_{z \rightarrow-z}$.

Proposition 5.23 (Givental's formula for toric DM stacks). The ancestor potential of $\mathfrak{X}_{\Sigma}$ is given by $\mathscr{A}_{\mathfrak{X}_{\boldsymbol{\Sigma}}, \operatorname{mir}(q, \chi)}=T_{-z \phi_{0}+R(z, \ldots, z)} \widehat{R} \mathscr{T}^{\otimes N}$ with $R=R_{q, \chi}$ above.

Proof. The mirror isomorphism Mir ${ }^{\text {an }}$ identifies the Gauss-Manin connection with the quantum connection, and it was shown in [27, Lemma 6.7] that $e^{F_{\mathbb{T}}(p) / z} \operatorname{Asym}_{p}$ gives a solution to the Gauss-Manin connection for each critical branch $p$ of $F_{\mathbb{T}}$. Let $\mathbf{U}$ denote the diagonal matrix whose entries are relative critical values of $F_{\mathbb{T}}$. It follows that $\widetilde{R}_{q, \chi} e^{-\mathbf{U} / z}$ gives a formal fundamental solution for mir* $\nabla$ in the sense of [48, Proposition 1.1], [109, Theorem 5.1] (our $R_{q, \chi}$ corresponds to $\Psi R$ in $[48,109]$; see also Proposition 6.1 below). We need to flip the sign of $z$ because we use the sign convention for the quantum connection opposite to [48, 109]. It now suffices to check that $R_{q, \chi}$ satisfies the classical limit condition at the large radius limit $q=0_{\boldsymbol{\Sigma}}$ given in [19, Lemma 6.5, equation (156), Remark 6.6], [109, Theorem 6.2] (which generalize [49, Theorem 9.1] to the orbifold setting). We can easily check that $R_{q, \chi}$ satisfies this condition by using the computation in [27, Proposition 6.9].

Remark 5.24. It has been observed by Givental [49] that the operator $R$ can be constructed by equivariant mirrors (for Fano toric manifolds).

Remark 5.25. Recall from Remark 4.29 that the asymptotic expansion is ambiguous up to sign. Therefore $R_{q, \chi}$ is ambiguous up to the right multiplication by a signed permutation matrix. The right-hand side of the Givental formula is, however, independent of the choice (see, e.g., [29, Proposition 4.3]).

Proof of Theorem 5.19. Let $R_{q, \chi}^{ \pm}$denote the $R$-operators for $\mathfrak{X}_{ \pm}$introduced above. By the construction of $U_{q, \chi}$ in Section 5.5.3, we have the following commutative diagram for $(q, \chi) \in \mathcal{U}_{0}^{\prime \text { ss }}$ (when we order the critical points of $F_{\mathbb{T}}$ appropriately):

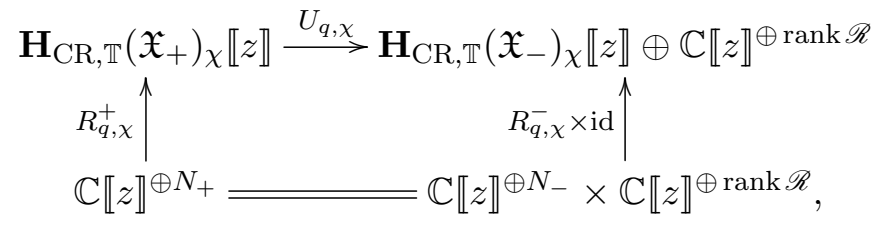

where $N_{ \pm}=\operatorname{dim} H_{\mathrm{CR}}^{*}\left(\mathfrak{X}_{ \pm}\right)$. The conclusion follows by Proposition 5.23 and the 'chain rule' of the Givental quantization (see, e.g., [29, Remark 3.22]).

\section{Formal decomposition and analytic lift}

We discuss the formal decomposition and its analytic lift for quantum D-modules or Brieskorn modules. This is known as the Hukuhara-Turrittin theorem for irregular differential equations. For mirrors of the small quantum cohomology of weak Fano compact toric stacks, the analytic lift is described explicitly in terms of oscillatory integrals. In this section, we restrict ourselves to the non-equivariant quantum cohomology and Brieskorn module, and assume that the toric stacks are compact.

\subsection{Hukuhara-Turrittin type result}

Recall that the non-equivariant quantum connection (or, equivalently, the non-equivariant Gauss-Manin connection) can be extended in the direction of $z$. The connection in the $z$ direction is of the form (see (2.11))

$$
\nabla_{z \frac{\partial}{\partial z}}=z \frac{\partial}{\partial z}-\frac{1}{z}\left(E \star_{\tau}\right)+\mu \text {. }
$$


When the quantum product $\star_{\tau}$ is semisimple, $\nabla$ decomposes over $\mathbb{C} \llbracket z \rrbracket$ into the sum of rank-one connections $d+d\left(\mathbf{u}_{i} / z\right), i=1, \ldots, N$ where $\mathbf{u}_{1}, \ldots, \mathbf{u}_{N}$ are eigenvalues of $E \star_{\tau}$. Moreover this formal decomposition admits an analytic lift over a certain angular sector: this is an instance of the Hukuhara-Turrittin theorem (see, e.g., [107, Theorem 12.3], [10, Theorem A], [95, Chapter II, Section 5.d] for more general statement). We state a version of this theorem following Hertling-Sevenheck [57, Section 8] and Galkin-Golyshev-Iritani [44, Section 2.5]. We restrict our attention to the quantum cohomology of toric stacks, but the discussion in this section can be equally applied to a general semisimple Frobenius manifold [40].

Let $\mathfrak{X}_{\Sigma}$ be a semiprojective smooth toric DM stack from Section 3.1.1. In this section (Section 6.1), we assume that $\mathfrak{X}_{\boldsymbol{\Sigma}}$ is compact but not necessarily weak Fano. Let $\mathcal{M}_{\mathrm{A}}\left(\mathfrak{X}_{\boldsymbol{\Sigma}}\right)$ be the non-equivariant Kähler moduli space of $\mathfrak{X}_{\Sigma}$ from Section 2.2. By the convergence result [27, Corollary 7.3], the non-equivariant quantum product is analytic over an open neighbourhood $V \subset\left[\mathcal{M}_{\mathrm{A}}\left(\mathfrak{X}_{\boldsymbol{\Sigma}}\right) / \mathrm{Pic}^{\mathrm{st}}\left(\mathfrak{X}_{\boldsymbol{\Sigma}}\right)\right]$ of the large radius limit point. This defines the (non-equivariant) quantum connection $\nabla$ over $V$ and the analytic quantum D-module (cf. Sections 2.3 and 4.4):

$$
\mathrm{QDM}^{\mathrm{an}}\left(\mathfrak{X}_{\boldsymbol{\Sigma}}\right)=\left(H_{\mathrm{CR}}^{*}\left(\mathfrak{X}_{\boldsymbol{\Sigma}}\right) \otimes \mathcal{O}_{\widetilde{V}}^{\mathrm{an}}[z], \nabla, P\right),
$$

where $\nabla$ is understood as being extended in the $z$-direction by $(2.11)$ and $\widetilde{V}$ is the preimage of $V$ in $\mathcal{M}_{\mathrm{A}}\left(\mathfrak{X}_{\boldsymbol{\Sigma}}\right) ; H_{\mathrm{CR}}^{*}\left(\mathfrak{X}_{\boldsymbol{\Sigma}}\right) \otimes \mathcal{O}_{\widetilde{V}}^{\text {an }}[z]$ is a $\mathrm{Pic}^{\text {st }}\left(\mathfrak{X}_{\boldsymbol{\Sigma}}\right)$-equivariant sheaf, which shall be regarded as a sheaf on the stack $V=\left[\widetilde{V} / \mathrm{Pic}^{\text {st }}\left(\mathfrak{X}_{\Sigma}\right)\right]$. We omitted the grading operator from the data because it can be recovered from the other data as $\mathrm{Gr}=\nabla_{\mathcal{E}}+\nabla_{z \frac{\partial}{\partial z}}+\frac{1}{2} \operatorname{dim} \mathfrak{X}_{\Sigma}$. We also write

$$
\overline{\mathrm{QDM}^{\mathrm{an}}}\left(\mathfrak{X}_{\boldsymbol{\Sigma}}\right)=\left(H_{\mathrm{CR}}^{*}\left(\mathfrak{X}_{\boldsymbol{\Sigma}}\right) \otimes \mathcal{O}_{\widetilde{V}}^{\mathrm{an}} \llbracket z \rrbracket, \nabla, P\right)
$$

for the quantum D-module completed in $z$. We set

$$
\begin{aligned}
& V^{\times}:=V \cap\left(\text { the image of the natural map } H_{\mathrm{CR}}^{*}\left(\mathfrak{X}_{\boldsymbol{\Sigma}}\right) \rightarrow\left[\mathcal{M}_{\mathrm{A}}\left(\mathfrak{X}_{\boldsymbol{\Sigma}}\right) / \operatorname{Pic}^{\mathrm{st}}\left(\mathfrak{X}_{\boldsymbol{\Sigma}}\right)\right]\right), \\
& V^{\mathrm{ss}}:=\left\{\tau \in V^{\times}:\left(H_{\mathrm{CR}}^{*}\left(\mathfrak{X}_{\boldsymbol{\Sigma}}\right), \star_{\tau}\right) \text { is semisimple as a ring }\right\} \subset V^{\times} .
\end{aligned}
$$

Here recall from Section 2.2 that $\mathcal{M}_{\mathrm{A}}\left(\mathfrak{X}_{\boldsymbol{\Sigma}}\right)$ is a partial compactification of $H_{\mathrm{CR}}^{*}\left(\mathfrak{X}_{\boldsymbol{\Sigma}}\right) / 2 \pi \mathbf{i}\left(\boldsymbol{\Lambda}^{\boldsymbol{\Sigma}}\right)^{\star}$ and hence we have a natural map $H_{\mathrm{CR}}^{*}\left(\mathfrak{X}_{\boldsymbol{\Sigma}}\right) \rightarrow \mathcal{M}_{\mathrm{A}}\left(\mathfrak{X}_{\boldsymbol{\Sigma}}\right) \rightarrow\left[\mathcal{M}_{\mathrm{A}}\left(\mathfrak{X}_{\boldsymbol{\Sigma}}\right) / \operatorname{Pic}^{\text {st }}\left(\mathfrak{X}_{\boldsymbol{\Sigma}}\right)\right]$. When $\mathfrak{X}_{\boldsymbol{\Sigma}}$ is compact, $V^{\text {ss }}$ is open and dense in $V^{\times}$[27, Remark 7.11]. The following proposition describes a Levelt-Turrittin normal form [95, Chapter II, Theorem 5.7] of the quantum connection in the semisimple case.

Proposition 6.1. Let $\tau_{0} \in V^{\mathrm{ss}}$ be a semisimple point. We have the following decomposition in an open neighbourhood of $\tau_{0}$ :

$$
\widehat{\Phi}: \quad \overline{\mathrm{QDM}^{\mathrm{an}}}\left(\mathfrak{X}_{\boldsymbol{\Sigma}}\right) \stackrel{\cong}{\longrightarrow} \bigoplus_{i=1}^{N}\left(\mathcal{O}^{\mathrm{an}} \llbracket z \rrbracket, d+d\left(\mathbf{u}_{i} / z\right), P_{\mathrm{std}}\right),
$$

where $\mathbf{u}_{1}, \ldots, \mathbf{u}_{N}$ are eigenvalues of $E_{\star_{\tau}}$ with $N=\operatorname{dim} H_{\mathrm{CR}}^{*}\left(\mathfrak{X}_{\boldsymbol{\Sigma}}\right)$ and $P_{\mathrm{std}}$ is the pairing given

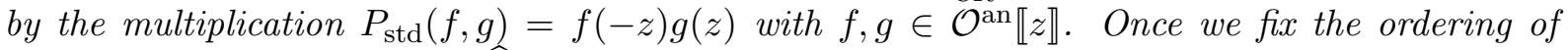
$\mathbf{u}_{1}, \ldots, \mathbf{u}_{N}$, the isomorphism $\Phi$ is unique up to a right multiplication by $\operatorname{diag}( \pm 1, \ldots, \pm 1)$.

Proof. When $\mathbf{u}_{1}, \ldots, \mathbf{u}_{N}$ are mutually distinct, this is discussed in [40, Lecture 4], [48, Proposition 1.1], [95, Chapter II, Theorem 5.7], [30, Proposition 7.2]. When $\mathbf{u}_{1}, \ldots, \mathbf{u}_{N}$ are not mutually distinct (but $\star_{\tau}$ is still semisimple), the existence of $\widehat{\Phi}$ was shown by Teleman [104, Theorem 8.15] (see also [18, Section 8]). That $\widehat{\Phi}$ depends analytically on $\tau$ (along the locus where some of $\mathbf{u}_{1}, \ldots, \mathbf{u}_{N}$ coalesce) was discussed in [44, Remark 2.5.7]. The pairing fixes the isomorphism $\widehat{\Phi}$ up to sign (on each factor), see the argument in [30, Proposition 7.2]. 
Remark 6.2. In this proposition, we do not require that eigenvalues of $E \star_{\tau}$ are mutually distinct. Note however that $\mathbf{u}_{1}, \ldots, \mathbf{u}_{N}$ form a co-ordinate system in a neighbourhood of a semisimple point $\tau_{0}$ [40, Lecture 3], and thus they are generically mutually distinct.

Remark 6.3. The isomorphism $\widehat{\Phi}$ modulo $z$ is given by a normalized idempotent basis of $\left(H_{\mathrm{CR}}^{*}\left(\mathfrak{X}_{\boldsymbol{\Sigma}}\right), \star_{\tau}\right)$, i.e., a basis $\left\{\Psi_{1}, \ldots, \Psi_{N}\right\}$ such that $\Psi_{i} \star_{\tau} \Psi_{j}$ is a non-zero scalar multiple of $\delta_{i, j} \Psi_{i}$ and that $\left(\Psi_{i}, \Psi_{j}\right)=\delta_{i, j}$.

In plain words, Proposition 6.1 means that the differential equation $\nabla_{z \frac{\partial}{\partial z}} f=0$ for a cohomology-valued function $f=f(z)$ admits a formal power series solution

$$
f=e^{-\mathbf{u}_{i} / z}\left(\Psi_{i, 0}+\Psi_{i, 1} z+\Psi_{i, 2} z^{2}+\cdots\right)
$$

with $\Psi_{i, n} \in H_{\mathrm{CR}}^{*}\left(\mathfrak{X}_{\boldsymbol{\Sigma}}\right)$, where $\Psi_{i, 0}=\Psi_{i}$ is the normalized idempotent in Remark 6.3. These formal power series are typically divergent. Over an appropriate angular sector in the $z$-plane, however, we can find an actual analytic solution whose asymptotic expansion is given by (6.3); moreover the actual solution with prescribed asymptotics is unique if the angle of the sector is bigger than $\pi$.

To state this analytic lift in a sheaf-theoretic language, we follow Sabbah [95] and introduce a sheaf $\mathcal{A}$ of "holomorphic" functions on the real blowup of $\mathbb{C}$. An (open) sector is a subset of $\mathbb{C}$ of the form $\left\{z \in \mathbb{C}^{\times}: \phi_{1}<\arg z<\phi_{2},|z|<\delta\right\}$ for some $\phi_{1}, \phi_{2} \in \mathbb{R}$ and $\delta \in(0, \infty]$. A holomorphic function $f(z)$ on the sector $I=\left\{z \in \mathbb{C}^{\times}: \phi_{1}<\arg z<\phi_{2},|z|<\delta\right\}$ is said to have the asymptotic expansion $f \sim \sum_{k=0}^{\infty} a_{k} z^{k}$ as $z \rightarrow 0$ along $I$ if for every $\epsilon>0$ and for every $m \geq 0$, there exists a constant $C_{\epsilon, m}>0$ such that

$$
\left|f(z)-\sum_{k=0}^{m} a_{k} z^{k}\right| \leq C_{\epsilon, m}|z|^{m+1}
$$

for all $z$ with $\phi_{1}+\epsilon \leq \arg z \leq \phi_{2}-\epsilon$ and $|z| \leq \delta / 2$.

Definition 6.4 ([95, Chapter II, Section 5.c]). Let $\widetilde{\mathbb{C}}:=[0, \infty) \times S^{1}$ denote the oriented real blowup of $\mathbb{C}$ at the origin. This is a smooth manifold with boundary and is equipped with the map $\pi: \widetilde{\mathbb{C}} \rightarrow \mathbb{C}, \pi\left(r, e^{\mathbf{i} \theta}\right)=r e^{\mathbf{i} \theta}$. Let $C_{\widetilde{\mathbb{C}}}^{\infty}$ denote the sheaf of complex-valued $C^{\infty}$-functions on $\widetilde{\mathbb{C}}$ and define $\mathcal{A}_{\widetilde{\mathbb{C}}}$ to be the subsheaf of $C_{\widetilde{\mathbb{C}}}^{\mathbb{C}}$ of germs annihilated by the Cauchy-Riemann operator $\bar{z} \frac{\partial}{\partial \bar{z}}=\frac{1}{2}\left(r \frac{\partial}{\partial r}+\mathbf{i} \frac{\partial}{\partial \theta}\right)$. Sections of $\mathcal{A}_{\widetilde{\mathbb{C}}}$ over the open set $\left\{\left(r, e^{\mathbf{i} \theta}\right) \in \widetilde{\mathbb{C}}: 0 \leq r<\delta, \phi_{1}<\theta<\phi_{2}\right\}$ are precisely those holomorphic functions $f(z)$ on the sector $\left\{z \in \mathbb{C}^{\times}: \phi_{1}<\arg z<\phi_{2},|z|<\delta\right\}$ which admit asymptotic expansions $f(z) \sim \sum_{k=0}^{\infty} a_{k} z^{k}$ along this sector.

The same construction works in families: for a complex manifold $M$, we similarly define the sheaf $\mathcal{A}_{M \times \widetilde{\mathbb{C}}}$ over $M \times \widetilde{\mathbb{C}}$ to be the subsheaf of $C_{M \times \widetilde{\mathbb{C}}}^{\infty}$ of germs annihilated by the CauchyRiemann operators $\bar{z} \frac{\partial}{\partial \bar{z}}, \bar{\partial}_{M}$.

The asymptotic expansion yields a map

$$
i_{\xi}^{-1} \mathcal{A}_{M \times \widetilde{\mathbb{C}}} \rightarrow \mathcal{O}_{M}^{\mathrm{an}} \llbracket z \rrbracket
$$

for any $\xi \in S^{1}$, where $i_{\xi}: M \cong M \times\{(0, \xi)\} \hookrightarrow M \times \widetilde{\mathbb{C}}$ is the inclusion. This map is known to be surjective (the Borel-Ritt lemma; see, e.g., [107]). The natural map $\pi: M \times \widetilde{\mathbb{C}} \rightarrow M \times \mathbb{C}$ is a map of ringed spaces, i.e., we have a map $\pi^{-1} \mathcal{O}_{M \times \mathbb{C}}^{\text {an }} \rightarrow \mathcal{A}_{M \times \widetilde{\mathbb{C}}}$ of sheaves of rings. In particular, we can define the pull-back of an $\mathcal{O}_{M \times \mathbb{C}^{-m o d u l e}}^{\text {an }} \mathcal{F}$ to be $\pi^{*} \mathcal{F}:=\pi^{-1} \mathcal{F} \otimes_{\pi^{-1} \mathcal{O}_{M \times \mathbb{C}}^{\text {an }}} \mathcal{A}_{M \times \widetilde{\mathbb{C}}}$.

For a multi-set $\left\{\mathbf{u}_{i}\right\}=\left\{\mathbf{u}_{1}, \ldots, \mathbf{u}_{N}\right\}$ of complex numbers, we say that a direction $e^{\mathbf{i} \phi} \in S^{1}$ or a phase $\phi$ is admissible for $\left\{\mathbf{u}_{i}\right\}$ if $e^{\mathbf{i} \phi}$ is not parallel to any non-zero difference $\mathbf{u}_{i}-\mathbf{u}_{j}$, i.e., $e^{\mathbf{i} \phi} \notin \mathbb{R}\left(\mathbf{u}_{i}-\mathbf{u}_{j}\right)$ for all $i, j$. 
Proposition 6.5. Let $\tau_{0} \in V^{\mathrm{ss}}$ be a semisimple point and let $e^{\mathbf{i} \phi} \in S^{1}$ be an admissible direction for the spectrum $\left\{\mathbf{u}_{1}^{0}, \ldots, \mathbf{u}_{N}^{0}\right\}$ of $E \star_{\tau_{0}}$. Let $\pi: V^{\mathrm{ss}} \times \widetilde{\mathbb{C}} \rightarrow V^{\mathrm{ss}} \times \mathbb{C}$ denote the oriented real blowup along $V^{\mathrm{ss}} \times\{0\}$. There exist an open neighbourhood $B$ of $\tau_{0}$ in $V^{\mathrm{ss}}$, a positive number $\epsilon>0$, and an isomorphism

$$
\Phi_{\phi}:\left.\quad \pi^{*} \operatorname{QDM}^{\mathrm{an}}\left(\mathfrak{X}_{\boldsymbol{\Sigma}}\right)\right|_{B \times I_{\phi}} \cong \bigoplus_{i=1}^{N}\left(\mathcal{A}_{B \times I_{\phi}}, d+d\left(\mathbf{u}_{i} / z\right)\right)
$$

over the sector $I_{\phi}=\left\{\left(r, e^{\mathrm{i} \theta}\right):|\theta-\phi|<\frac{\pi}{2}+\epsilon\right\}$ such that $\Phi_{\phi}$ induces, via (6.4), the formal decomposition $\widehat{\Phi}$ in Proposition 6.1. Here we exclude the data of the pairing from $\operatorname{QDM}^{\mathrm{an}}\left(\mathfrak{X}_{\boldsymbol{\Sigma}}\right)$. Moreover such a $\Phi_{\phi}$ is unique. We call $\Phi_{\phi}$ the analytic lift of $\widehat{\Phi}$.

Proof. In the case where $\left\{\mathbf{u}_{1}^{0}, \ldots, \mathbf{u}_{N}^{0}\right\}$ are pairwise distinct, similar results are given in [107, Theorem 12.3], [10, Theorem A], [40, Theorem 4.2], [95, Chapter II, Theorem 5.12]. We closely follow Hertling-Sevenheck [57, Lemma 8.3] for the formulation. The general case follows from [44, Proposition 2.5.1], where a fundamental solution matrix $Y_{\phi}(\tau, z)$ for $\nabla$ with the asymptotics $Y_{\phi}(\tau, z) e^{-\mathbf{U} / z} \rightarrow\left(\Psi_{1}, \ldots, \Psi_{N}\right)$ as $z \rightarrow 0$ along the sector $I_{\phi}$ is constructed for $\tau \in B$. Here $\mathbf{U}=\operatorname{diag}\left[\mathbf{u}_{1}, \ldots, \mathbf{u}_{N}\right]$ and $\Psi_{i}$ is the normalized idempotent in Remark 6.3.

Remark 6.6. The uniqueness of the analytic lift $\Phi_{\phi}$ is ensured by the fact that the angle of the sector $I_{\phi}$ is bigger than $\pi$ : see [10, Remark 1.4]. The lift $\Phi_{\phi}$ depends on $\tau_{0}$ and $e^{\mathbf{i} \phi}$, and it depends continuously on $\left(\tau_{0}, e^{\mathbf{i} \phi}\right)$ unless $\left(\tau_{0}, e^{\mathbf{i} \phi}\right)$ crosses the locus where $e^{\mathbf{i} \phi}$ is non-admissible for the spectrum of $E \star_{\tau_{0}}$.

Remark 6.7. The analytic lift $\Phi_{\phi}$ preserves the pairing in the following sense: consider the analytic lift $\Phi_{\phi+\pi}$ associated with the opposite direction $-e^{\mathbf{i} \phi}$, then

$$
P\left(s_{-}, s_{+}\right)(x, z)=\sum_{i=1}^{N} \Phi_{\phi+\pi}^{i}\left(s_{-}\right)(x,-z) \Phi_{\phi}^{i}\left(s_{+}\right)(x, z)
$$

for sections $s_{-}, s_{+}$of $\pi^{*} \mathrm{QDM}^{\mathrm{an}}\left(\mathfrak{X}_{\boldsymbol{\Sigma}}\right)$, respectively, over $B \times I_{\phi+\pi}, B \times I_{\phi}$. This follows from the fact that the asymptotic expansions of both sides coincide by Proposition 6.5, and that the pairings are flat.

Remark 6.8. For a thorough discussion on the isomonodromic deformation theory of irregular differential equations (of Poincaré rank 1) with coalescing eigenvalues $\mathbf{u}_{1}, \ldots, \mathbf{u}_{N}$, see CottiDubrovin-Guzzetti [33].

\subsection{Asymptotic basis and marked reflection system}

The sectorial decomposition in Proposition 6.5 gives rise to a linear algebraic data which we call the marked reflection system [44, Section 4.3]; this notion is equivalent to the central connection matrix together with canonical co-ordinates in Dubrovin's theory [40, Lecture 4]. We briefly review it for our later purposes.

Introduce a pairing $[\cdot, \cdot)$ on the $\mathbb{C}$-vector space $H_{\mathrm{CR}}^{*}\left(\mathfrak{X}_{\Sigma}\right)$ by

$$
[\alpha, \beta):=\frac{1}{(2 \pi)^{n}}\left(e^{\pi \mathbf{i} \mu} e^{-\pi \mathbf{i}_{1}\left(\mathfrak{X}_{\boldsymbol{\Sigma}}\right)} \alpha, \beta\right),
$$

where $(\cdot, \cdot)$ is the orbifold Poincaré pairing $(2.2)$.

Recall the fundamental solution $L(\tau, z) z^{-\mu} z^{c_{1}\left(\mathfrak{X}_{\Sigma}\right)}$ of the quantum connection introduced in Section 2.4. The map $\alpha \mapsto(2 \pi)^{-n / 2} L(\tau, z) z^{-\mu} z^{c_{1}\left(\mathfrak{X}_{\boldsymbol{\Sigma}}\right)} \alpha$ intertwines the pairing $[\cdot, \cdot)$ on $H_{\mathrm{CR}}^{*}\left(\mathfrak{X}_{\boldsymbol{\Sigma}}\right)$ with the orbifold Poincaré pairing in the sense that

$$
\left(s_{1}\left(\tau, e^{-\mathbf{i} \pi} z\right), s_{2}(\tau, z)\right)=\left[\alpha_{1}, \alpha_{2}\right),
$$


when $s_{i}(\tau, z)=(2 \pi)^{-n / 2} L(\tau, z) z^{-\mu} z^{c_{1}\left(\mathfrak{X}_{\boldsymbol{\Sigma}}\right)} \alpha_{i}$ (where $n=\operatorname{dim} \mathfrak{X}_{\boldsymbol{\Sigma}}$ as usual), see [62, (20)]. The pairing $[\cdot, \cdot)$ is also related to the Euler pairing $\chi(\cdot, \cdot)$ on the $K$-group by (2.13), i.e.,

$$
\chi\left(V_{1}, V_{2}\right)=\left[\alpha_{1}, \alpha_{2}\right),
$$

when $\alpha_{i}=\widehat{\Gamma}_{\mathfrak{X}_{\boldsymbol{\Sigma}}} \cup(2 \pi \mathbf{i})^{\operatorname{deg}_{0} / 2} \operatorname{inv}^{*} \widetilde{\operatorname{ch}}\left(V_{i}\right)$ and $V_{i} \in K\left(\mathfrak{X}_{\boldsymbol{\Sigma}}\right)$.

Let $\tau_{0} \in V^{\mathrm{ss}}$ be a semisimple point and let $\phi$ be an admissible phase for the eigenvalues of $E \star_{\tau_{0}}$. Let $\Phi_{\phi}$ be the sectorial decomposition associated with $\tau_{0}$ and $\phi$ as in Proposition 6.5:

$$
\Phi_{\phi}:\left.\pi^{*} \mathrm{QDM}^{\mathrm{an}}\left(\mathfrak{X}_{\boldsymbol{\Sigma}}\right)\right|_{B \times I_{\phi}} \cong \bigoplus_{i=1}^{N}\left(\mathcal{A}_{B \times I_{\phi}}, d+d\left(\mathbf{u}_{i} / z\right)\right) .
$$

We choose a base point $\tau_{\star} \in V^{\times}$corresponding to a real cohomology class and choose a path connecting $\tau_{\star}$ and $\tau_{0}$ in $V^{\times}$(see Remark 2.8 for the choice of a base point). Let $s_{i}$ be the flat section of $\mathrm{QDM}^{\mathrm{an}}\left(\mathfrak{X}_{\boldsymbol{\Sigma}}\right)$ on a neighbourhood of $\left(\tau_{0}, e^{\mathbf{i} \phi}\right)$ satisfying $\Phi_{\phi}\left(s_{i}\right)=e^{-\mathbf{u}_{i} / z} e_{i}$, where $e_{i}$ is the $i$ th standard basis of $\mathcal{A}_{B \times I_{\phi}}^{\oplus N}$. Then we have vectors $v_{i} \in H_{\mathrm{CR}}^{*}\left(\mathfrak{X}_{\Sigma}\right)$ such that

$$
s_{i}(\tau, z)=(2 \pi)^{-n / 2} L(\tau, z) z^{-\mu} z^{c_{1}\left(\mathfrak{X}_{\boldsymbol{\Sigma}}\right)} v_{i},
$$

where the determination of the fundamental solution is given by $\arg z=\phi$ at $z=e^{\mathbf{i} \phi}$ and the chosen path (see Remark 2.8). The marked reflection system associated with $\tau_{0}, \phi$ (and the path from $\tau_{\star}$ to $\left.\tau_{0}\right)$ is a tuple $\left(H_{\mathrm{CR}}^{*}\left(\mathfrak{X}_{\boldsymbol{\Sigma}}\right),[\cdot, \cdot),\left\{v_{1}, \ldots, v_{N}\right\}, m, e^{\mathbf{i} \phi}\right)$, where

- $\left\{v_{1}, \ldots, v_{N}\right\}$ is the basis of $H_{\mathrm{CR}}^{*}\left(\mathfrak{X}_{\boldsymbol{\Sigma}}\right)$ defined as above, called the asymptotic basis;

- $m:\left\{v_{1}, \ldots, v_{N}\right\} \rightarrow \mathbb{C}$ is the map given by $m\left(v_{i}\right)=\mathbf{u}_{i}$, called the marking.

The asymptotic basis satisfies the following semiorthogonality condition [44, Proposition 2.6.4, Section 4.4]

$$
\left[v_{i}, v_{j}\right)= \begin{cases}1, & \text { if } i=j, \\ 0, & \text { if } i \neq j \text { and } \Im\left(e^{-\mathbf{i} \phi} \mathbf{u}_{i}\right) \leq \Im\left(e^{-\mathbf{i} \phi} \mathbf{u}_{j}\right) .\end{cases}
$$

Because of the ambiguity of $\widehat{\Phi}$ in Proposition 6.1 , the asymptotic basis $\left\{v_{1}, \ldots, v_{n}\right\}$ is not a priori ordered and each $v_{i}$ is determined up to sign $v_{i} \rightarrow \pm v_{i}$. On the other hand, given a phase $\phi$, we can order $\left\{v_{i}\right\}$ in such a way that $\Im\left(e^{-\mathbf{i} \phi} \mathbf{u}_{1}\right) \geq \Im\left(e^{-\mathbf{i} \phi} \mathbf{u}_{2}\right) \geq \cdots \geq \Im\left(e^{\mathbf{i} \phi} \mathbf{u}_{N}\right)$; then the Gram matrix $\left(\left[v_{i}, v_{j}\right)\right)$ is upper-triangular with diagonal entries all equal to one, and gives the Stokes matrix of the quantum connection at the irregular singular point $z=0$ (see [40, Lecture 4], [44, Proposition 2.6.4]).

When $\tau_{0}$ and $\phi$ vary and cross the locus where the corresponding eigenvalues $\left\{\mathbf{u}_{i}\right\}$ become non-admissible for $\phi$, the corresponding marked reflection system undergoes mutation. We refer to [44, Section 4.2] for the full details of the deformation theory of marked reflection systems. We illustrate an example of mutation in Fig. 7; the figure describes a typical procedure where $\left\{\mathbf{u}_{i}\right\}$ varies in the configuration space of $N$ points in $\mathbb{C}$ and crosses the wall of nonadmissible configurations. In the picture, we drew the half-ray $\mathbf{u}_{j}+\mathbb{R}_{\geq 0} e^{\mathbf{i} \phi}$ from each $\mathbf{u}_{j}$ to show the direction $e^{\mathbf{i} \phi}$. Suppose that the asymptotic basis $\left\{v_{1}, \ldots, v_{N}\right\}$ is marked by $\left\{\mathbf{u}_{1}, \ldots, \mathbf{u}_{N}\right\}$ in the leftmost picture, i.e., $\mathbf{u}_{j}=m\left(v_{j}\right)$. We assume that the basis is ordered so that $\Im\left(e^{-\mathbf{i} \phi} \mathbf{u}_{1}\right)>$ $\Im\left(e^{-\mathbf{i} \phi} \mathbf{u}_{2}\right)>\cdots>\Im\left(e^{-\mathbf{i} \phi} \mathbf{u}_{N}\right)$. After passing through the non-admissible configuration in the middle picture, the vector $v_{i}$ marked by $\mathbf{u}_{i}$ is transformed into

$$
v_{i}^{\prime}=v_{i}-\left[v_{i}, v_{i+1}\right) v_{i+1}
$$


and the other vectors remain the same (i.e., the marking is given by $v_{i}^{\prime} \mapsto \mathbf{u}_{i}$ and $v_{j} \mapsto \mathbf{u}_{j}$ for $j \neq i$ in the rightmost picture). This is called the right mutation of $v_{i}$ with respect to $v_{i+1}$. The inverse procedure is the left mutation of $v_{i}^{\prime}$ with respect to $v_{i+1}$ :

$$
v_{i}=v_{i}^{\prime}-\left[v_{i+1}, v_{i}^{\prime}\right) v_{i+1}
$$

The two operations $(6.6),(6.7)$ are inverse to each other because of the semiorthogonality condition (6.5).

Remark 6.9. The result [62, Theorem 4.11] implies that the asymptotic basis $\left\{v_{1}, \ldots, v_{N}\right\}$ for the quantum cohomology of weak-Fano, compact toric stacks $\mathfrak{X}_{\Sigma}$ is of the form

$$
v_{i}=\widehat{\Gamma}_{\mathfrak{X}_{\Sigma}} \cup(2 \pi \mathbf{i})^{\operatorname{deg}_{0} / 2} \operatorname{inv}^{*} \widetilde{\operatorname{ch}}\left(V_{i}\right)
$$

for some classes $V_{i} \in K\left(\mathfrak{X}_{\boldsymbol{\Sigma}}\right)$ in the $K$-group, i.e., the corresponding flat section $s_{i}$ lies in the $\widehat{\Gamma}$ integral structure (see also Proposition 7.8). The Gamma conjecture II [44, Section 4.6] (recently proved by [41] for Fano toric manifolds) says that $V_{i}$ comes from a full exceptional collection in the derived category of coherent sheaves. In this situation, mutation of asymptotic basis corresponds to that of full exceptional collections.

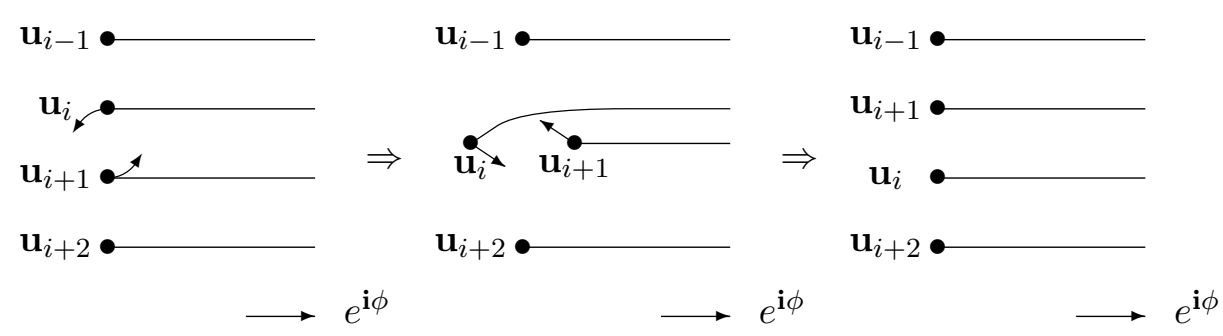

Figure 7. Right mutation: the "wall-crossing" from the left picture to the right one in the configuration space yields a right mutation of $v_{i}$.

\subsection{Sectorial decomposition of the Brieskorn module}

In this section, we describe the Hukuhara-Turrittin sectorial decomposition (Propositions 6.1 and 6.5) explicitly for the non-equivariant Brieskorn modules. First we observe that the analytified Brieskorn module $\overline{\mathrm{Bri}^{\mathrm{an}}}(F)_{\boldsymbol{\Sigma}}$ (Definition 4.21) admits a formal decomposition over the locus where $F$ has only non-degenerate critical points, via the formal asymptotic expansion in Section 4.3.3. When a toric stack $\mathfrak{X}_{\boldsymbol{\Sigma}}$ is weak Fano, the Brieskorn module $\operatorname{Bri}(F)$ over the small quantum cohomology locus of $\mathfrak{X}_{\boldsymbol{\Sigma}}$ has the expected rank equal to $\operatorname{dim} H_{\mathrm{CR}}^{*}\left(\mathfrak{X}_{\boldsymbol{\Sigma}}\right)$ and gives a fully analytic (i.e., analytic both in the $\mathcal{M}$ direction and in the $z$-direction) $\mathrm{D}$-module mirror to the small quantum D-module of $\mathfrak{X}_{\Sigma}$. In this case, we can describe the analytic lift of the formal decomposition using oscillatory integrals.

\subsubsection{Formal decomposition of the Brieskorn module}

Let $\mathfrak{X}_{\boldsymbol{\Sigma}}$ be a toric stack with $\boldsymbol{\Sigma} \in \mathfrak{F a n}(S)$ and let $(\operatorname{pr}: \mathcal{Y} \rightarrow \mathcal{M}, F)$ denote the LG model from Definition 3.6. Let $\mathcal{B} \subset \mathcal{Y}, \mathcal{U} \subset \mathcal{M}_{\mathbb{T}}=\mathcal{M} \times$ Lie $\mathbb{T}$ be open neighbourhoods of (respectively) $\tilde{0}_{\boldsymbol{\Sigma}}$ and $\left(0_{\boldsymbol{\Sigma}}, 0\right)$ as in Corollary 4.20. Recall that the analytified equivariant Brieskorn module $\overline{\operatorname{Bri}_{\mathbb{T}}^{\mathrm{an}}}(F)_{\Sigma}$ is defined over $\mathcal{U}$ and its non-equivariant version $\overline{\operatorname{Bri}^{\mathrm{an}}}(F)_{\boldsymbol{\Sigma}}$ is defined over 
$\mathcal{V}=\mathcal{U} \cap(\mathcal{M} \times\{0\})$ (see Definition 4.21). When $\mathfrak{X}_{\Sigma}$ is compact, the family of relative critical points of the LG potential $F$

$$
\mathrm{Cr}:=\left\{p \in \mathcal{B}: x_{1} \frac{\partial F}{\partial x_{1}}(p)=\cdots=x_{n} \frac{\partial F}{\partial x_{n}}(p)=0\right\} \stackrel{\mathrm{pr}}{\rightarrow} \mathcal{V}
$$

is a finite morphism whose generic fibre is reduced (see [62, Proposition 3.10]), i.e., there exists an open dense subset $\mathcal{V}^{\text {ss }}$ of $\mathcal{V}$ such that for any $q \in \mathcal{V}^{\text {ss }},\left.F\right|_{\mathcal{B} \cap \operatorname{pr}^{-1}(q)}$ has only non-degenerate critical points. We set $\mathrm{Cr}^{\mathrm{ss}}:=\mathrm{Cr} \cap \mathrm{pr}^{-1}\left(\mathcal{V}^{\mathrm{ss}}\right)$. By definition, $\mathrm{pr}: \mathrm{Cr}^{\mathrm{ss}} \rightarrow \mathcal{V}^{\mathrm{ss}}$ is a finite covering. The following result describes a formal decomposition parallel to Proposition 6.1 for the analytified Brieskorn module.

Proposition 6.10. The formal asymptotic expansion Asym in Definition 4.28 defines an isomorphism

$$
\text { Asym: }\left.\quad \overline{\operatorname{Bri}^{\mathrm{an}}}(F)_{\Sigma}\right|_{\mathcal{V}^{\mathrm{ss}}} \cong \operatorname{pr}_{*}\left(\mathcal{O}_{\mathrm{Cr}^{\mathrm{ss}}}^{\mathrm{an}} \llbracket z \rrbracket \otimes \text { ori }\right),
$$

where ori is the $\mu_{2}$-local system over $\mathrm{Cr}^{\mathrm{ss}}$ defined by the monodromy of the square root $\sqrt{\operatorname{det}\left(h_{i, j}\right)}$ of the logarithmic Hessian of $F$ (see (4.11) for $h_{i, j}$ ). This map identifies the Gauss-Manin connection $\nabla$ with $d+d\left(\left.F\right|_{\mathrm{Cr}^{\mathrm{ss}}} / z\right) \wedge$ and the higher residue pairing with the diagonal pairing:

$$
P_{\mathrm{std}}(f, g)(x)=\sum_{p \in \mathrm{pr}^{-1}(x) \cap \mathrm{Cr}^{\mathrm{ss}}} f(p,-z) g(p, z)
$$

where $f=f(x, z), g=g(x, z) \in \operatorname{pr}_{*}\left(\mathcal{O}_{\mathrm{Cr}}^{\mathrm{an}} \llbracket z \rrbracket\right)$.

Proof. As we remarked in Remark 4.29, we need to choose a square root of the Hessian when defining the formal asymptotic expansion; hence we need the $\mu_{2}$-local system ori. Along $z=0$, the map Asym is given by $\left.\phi \cdot \omega \mapsto \phi\right|_{\mathrm{Cr}} ^{\mathrm{ss}} /\left(\left|\mathbf{N}_{\mathrm{tor}}\right| \sqrt{\operatorname{det}\left(h_{i, j}\right)}\right)$. On the other hand,

$$
\begin{aligned}
\left.\overline{\mathrm{Bri}^{\mathrm{an}}}(F)_{\Sigma}\right|_{z=0} & \cong \operatorname{pr}_{*}\left(\mathcal{O}_{\mathcal{B}}^{\mathrm{an}} \llbracket z \rrbracket /\left(\chi_{1}, \ldots, \chi_{n}, z\right) \mathcal{O}_{\mathcal{B}}^{\mathrm{an}} \llbracket z \rrbracket\right) \\
& \cong \operatorname{pr}_{*}\left(\mathcal{O}_{\mathcal{B}}^{\mathrm{an}} /\left(x_{1} \frac{\partial F}{\partial x_{1}}, \ldots, x_{n} \frac{\partial F}{\partial x_{n}}\right)\right) \cong \operatorname{pr}_{*} \mathcal{O}_{\mathrm{Cr}}^{\mathrm{an}},
\end{aligned}
$$

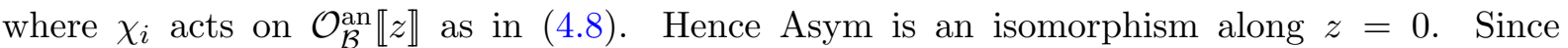
both sides are locally free $\mathcal{O}_{\mathcal{V} s s} \llbracket z \rrbracket$-modules, Asym is an isomorphism. That Asym identifies the connections follows from [27, Lemma 6.7]. That Asym identifies the pairings is obvious from Definition 4.30 .

Remark 6.11. The eigenvalues $\mathbf{u}_{1}, \ldots, \mathbf{u}_{N}$ of $E_{\star_{\tau}}$ in Proposition 6.1 correspond to critical values of $F$.

\subsubsection{Brieskorn module over the small quantum cohomology locus}

To discuss an analytic lift of the above formal decomposition, we restrict the Brieskorn module to the small quantum cohomology locus. We assume that $\mathfrak{X}_{\Sigma}$ is a weak Fano (i.e., $-K_{\mathfrak{X}_{\Sigma}}$ is nef) and compact toric stack. Furthermore, we assume that

$$
S_{-}:=S \cap \Delta=\{b \in S: \bar{b} \in \Delta\} \text { generates } \mathbf{N} \text { as a group, }
$$

where $\Delta \subset \mathbf{N}_{\mathbb{R}}$ denotes the convex hull of ray vectors $\{\bar{b}: b \in R(\boldsymbol{\Sigma})\}$. The compactness implies that $\Delta$ contains the origin in its interior and the weak-Fano condition implies that all rays 
$b \in R(\boldsymbol{\Sigma})$ lie in the boundary of $\Delta$. By replacing $S$ with $S_{-}=S \cap \Delta$ in the construction of the (partially compactified) LG model in Section 3.2, we obtain an LG model

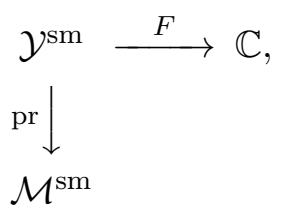

which we call the $L G$ model mirror to the small quantum cohomology of $\mathfrak{X}_{\boldsymbol{\Sigma}}$. We also call $\mathcal{M}^{\text {sm }}$ the small quantum cohomology locus of $\mathfrak{X}_{\boldsymbol{\Sigma}}$. Under the mirror map, $\mathcal{M}^{\mathrm{sm}}$ maps to $H_{\overline{\mathrm{CR}}}^{\leq 2}\left(\mathfrak{X}_{\boldsymbol{\Sigma}}\right)$.

Lemma 6.12. The total space $\mathcal{Y}^{\mathrm{sm}}$ is a closed toric substack of $\mathcal{Y}$ corresponding to the cone $\left(\mathbb{R}_{\geq 0}\right)^{S \backslash S_{-}}$of $\widetilde{\Xi}$; similarly $\mathcal{M}^{\mathrm{sm}}$ is a closed toric substack of $\mathcal{M}$ corresponding to the cone $D\left(\left(\mathbb{R}_{\geq 0}\right)^{S \backslash S_{-}}\right) \in \Xi$. Moreover, we have the pull-back diagram:

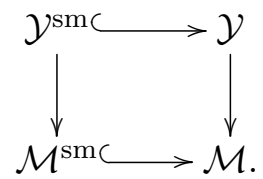

Proof. Let $\widetilde{\Xi}_{-}$denote the fan defining $\mathcal{Y}^{\text {sm }}$. Recall that maximal cones of $\widetilde{\Xi}$ are in one-to-one correspondence with stacky fans adapted to $S$; likewise, maximal cones of $\widetilde{\Xi}_{-}$are in one-to-one correspondence with stacky fans adapted to $S_{-}$. Thus the set of maximal cones of $\widetilde{\Xi}_{-}$can be identified with a subset of the set of maximal cones of $\widetilde{\Xi}$. We can see that this subset consists of maximal cones $\mathrm{CPL}_{+}(\boldsymbol{\Sigma})$ of $\widetilde{\Xi}$ that contain $\left(\mathbb{R}_{>0}\right)^{S \backslash S_{-}}$as a face; moreover the corresponding maximal cone of $\widetilde{\Xi}_{-}$is given by the image of $\mathrm{CPL}_{+}(\boldsymbol{\Sigma})$ under the projection $\left(\mathbb{R}^{S}\right)^{\star} \rightarrow\left(\mathbb{R}^{S_{-}}\right)^{\star}$. Therefore $\widetilde{\Xi}_{-}$is a fan obtained as the star of the cone $\left(\mathbb{R}_{\geq 0}\right)^{S \backslash S_{-}}$in $\widetilde{\Xi}$. This shows the first statement. A similar argument shows that $\mathcal{M}^{\mathrm{sm}}$ is a closed toric substack corresponding to $D\left(\left(\mathbb{R}_{\geq 0}\right)^{S \backslash S_{-}}\right)$. To see the pull-back diagram, we recall the description of the uniformizing chart in (3.21). When $\boldsymbol{\Sigma}$ is adapted to $S_{-}, G(\boldsymbol{\Sigma})$ contains $S \backslash S_{-}$. In the local chart associated with $\boldsymbol{\Sigma}$, the diagram (6.10) is of the form

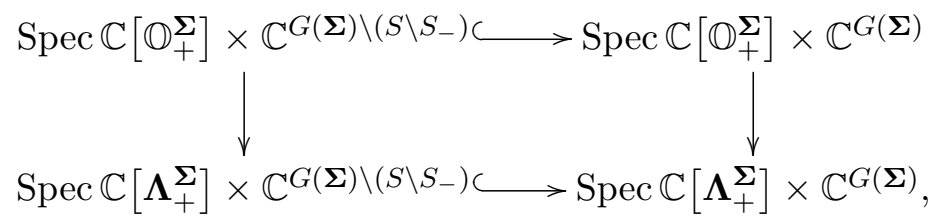

which is clearly a pull-back diagram.

Remark 6.13. The small quantum cohomology locus $\mathcal{M}^{\text {sm }} \subset \mathcal{M}$ depends on the choice of $\Sigma \in \mathfrak{F a n}(S)$.

Remark 6.14. The condition (6.8) ensures that Assumption 3.1 holds for $S_{-}=S \cap \Delta$. This condition is, however, not necessary at this point; we can define $\mathcal{Y}^{\text {sm }}$ (or $\mathcal{M}^{\text {sm }}$ ) as the substack corresponding to $\left(\mathbb{R}_{\geq 0}\right)^{S \backslash S_{-}}$(resp. to $D\left(\left(\mathbb{R}_{\geq 0}\right)^{S \backslash S_{-}}\right)$). We shall need this condition later when we apply the results on the $\widehat{\Gamma}$-integral structure for toric stacks in [62] (see Section 3.1.4 ibid where the same assumption was made).

We observe that the non-equivariant Brieskorn module $\operatorname{Bri}(F)$ over $\mathcal{M}^{\text {sm }}$ already has the expected rank; hence the completion and the analytification studied in Sections 4.2-4.3 are unnecessary over $\mathcal{M}^{\text {sm }}$ in the weak-Fano case. 
Proposition 6.15. Let $\mathfrak{X}_{\boldsymbol{\Sigma}}$ be a weak Fano compact toric stack satisfying (6.8). There exists a Zariski-open subset $\mathcal{M}^{\mathrm{sm} \text {,lf }}$ of $\mathcal{M}^{\mathrm{sm}}$ containing $0_{\boldsymbol{\Sigma}}$ such that the non-equivariant Brieskorn module $\left.\operatorname{Bri}(F)\right|_{\mathcal{M}^{\mathrm{sm}, \text { If }}}$ is a locally free $\mathcal{O}_{\mathcal{M}^{\mathrm{sm}, \text { If }} \times \mathbb{C}_{z}}$-module of rank $\operatorname{dim} H_{\mathrm{CR}}^{*}\left(\mathfrak{X}_{\boldsymbol{\Sigma}}\right)$.

Proof. This essentially follows from a result of Mann-Reichelt [81, Theorem 4.10] on the GKZ system; we give a proof of a more general statement (including the equivariant case and without assumption (6.8)) in Appendix A.

Let $\mathcal{V} \subset \mathcal{M}$ denote the base of the analytified Brieskorn module $\overline{\operatorname{Bri}^{\mathrm{an}}}(F)_{\boldsymbol{\Sigma}}$ as before. This is an analytic open neighbourhood of $0_{\boldsymbol{\Sigma}}$.

\section{Proposition 6.16.}

(1) $\operatorname{Set} \mathcal{V}^{\mathrm{sm}}=\mathcal{V} \cap \mathcal{M}^{\mathrm{sm}, \mathrm{lf}}$. The natural map

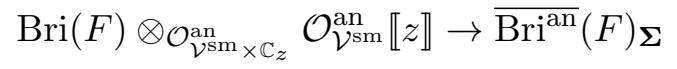

is an isomorphism.

(2) The mirror isomorphism Mir $\left.\right|_{\chi=0}$ in Proposition 4.11 extends to an isomorphism

$$
\left.\operatorname{Bri}(F)\right|_{\mathcal{V}^{\text {sm }}} \cong \operatorname{mir}^{*} \operatorname{QDM}^{\text {an }}(\mathfrak{X})
$$

over an open neighbourhood $\mathcal{V}^{\mathrm{sm} \prime} \subset \mathcal{V}^{\mathrm{sm}}$ of $0_{\boldsymbol{\Sigma}}$, where mir: $\mathcal{V}^{\mathrm{sm} \prime} \rightarrow V$ is the analytic mirror map (see Section 4.4 for the convergence of the mirror map).

Proof. For Part (1), it suffices to show that the map is an isomorphism along $z=0$. As discussed in the proof of Proposition 6.10, the analytified Brieskorn module along $z=0$ is isomorphic to $\mathrm{pr}_{*} \mathcal{O}_{\mathrm{Cr}}^{\text {an }}$. Thus the natural map in (1) is surjective along $z=0$; it is an isomorphism since the ranks are the same. Part (2) follows from the convergence of the $I$-function in the weak-Fano case, see, e.g., [62, Proposition 4.8].

\subsubsection{Analytic lift of the formal decomposition}

We continue to assume that $\mathfrak{X}_{\boldsymbol{\Sigma}}$ is a compact, weak-Fano toric stack and that the condition (6.8) holds. Consider the LG model (6.9) over $\mathcal{M}^{\mathrm{sm}}$. As a toric stack, $\mathcal{M}^{\text {sm }}$ contains the open dense torus orbit ${ }^{17}$

$$
\mathcal{M}^{\mathrm{sm}, \times}=\left(\mathbb{L}^{\prime}\right)^{\star} \otimes \mathbb{C}^{\times} \subset \mathcal{M}^{\mathrm{sm}},
$$

where $\mathbb{L}^{\prime}=\operatorname{Ker}\left(\mathbb{Z}^{S_{-}} \rightarrow \mathbf{N}\right)$ is the lattice appearing in the leftmost term of the extended fan sequence (3.3) with $S$ replaced with $S_{-}=S \cap \Delta$. The fibre of pr: $\mathcal{Y}^{\mathrm{sm}} \rightarrow \mathcal{M}^{\text {sm }}$ at a point $q \in \mathcal{M}^{\mathrm{sm}, \times}$ is isomorphic to $\operatorname{Hom}\left(\mathbf{N}, \mathbb{C}^{\times}\right)$(i.e., the fibre of the uncompactified LG model, see Section 3.2). In fact, since $\Delta$ contains the origin in its interior, we have a linear relation $\sum_{b \in S_{-}} \lambda_{b} b=0$ with $\lambda_{b} \in \mathbb{Z}_{>0}$; this shows that $\prod_{b \in S_{-}} u_{b}^{\lambda_{b}}=q^{\lambda} \neq 0$ on the fibre at $q \in \mathcal{M}^{\mathrm{sm}, \times}$ and in particular that $u_{b} \neq 0$ for all $b \in S_{-}$.

The locally freeness of $\left.\operatorname{Bri}(F)\right|_{\mathcal{M}^{\text {sm,lf }}}$ in Proposition 6.15 implies (by the restriction to $z=0$ ) that the family of relative critical points of $F$

$$
\mathrm{Cr}^{\mathrm{sm}}:=\left\{p \in \mathcal{Y}^{\mathrm{sm}}: x_{1} \frac{\partial F}{\partial x_{1}}(p)=\cdots=x_{n} \frac{\partial F}{\partial x_{n}}(p)=0\right\} \stackrel{\mathrm{pr}}{\rightarrow} \mathcal{M}^{\mathrm{sm}}
$$

\footnotetext{
${ }^{17}$ The following discussion works if we replace $\mathcal{M}^{\mathrm{sm}, \times}$ with the slightly bigger subspace $\left\{q \in \mathcal{M}_{\Sigma}^{\mathrm{sm}}\right.$ : $q^{\lambda} \neq 0$ for all $\left.\lambda \in \Lambda_{+}^{\Sigma}\right\}$ : this bigger space parametrizes Laurent polynomials with Newton polytope $\Delta$.
} 
is finite flat of degree $N=\operatorname{dim} H_{\mathrm{CR}}^{*}\left(\mathfrak{X}_{\boldsymbol{\Sigma}}\right)$ over the base $\mathcal{M}^{\text {sm,lf }}$. The generic fibre of the family $\mathrm{Cr}^{\mathrm{sm}} \rightarrow \mathcal{M}^{\mathrm{sm}}$ is reduced [62, Proposition 3.10], and thus

$$
\mathcal{M}^{\mathrm{sm}, \mathrm{ss}}=\left\{q \in \mathcal{M}^{\mathrm{sm}, \times} \cap \mathcal{M}^{\mathrm{sm}, \mathrm{lf}}:\left.F\right|_{\mathrm{pr}^{-1}(q)} \text { has only non-degenerate critical points }\right\}
$$

is a non-empty Zariski open subset, where "ss" means semisimplicity.

For $q \in \mathcal{M}^{\mathrm{sm}, \mathrm{ss}}$, let $\left\{c_{1}, \ldots c_{N}\right\}$ be the set of (mutually distinct) critical points of $F_{q}:=$ $\left.F\right|_{\mathrm{pr}^{-1}(q)}$, and let $\mathbf{u}_{i}=F_{q}\left(c_{i}\right)$ be the critical value. For an admissible phase $\phi$ for $\left\{\mathbf{u}_{1}, \ldots, \mathbf{u}_{N}\right\}$, let $\Gamma_{i}^{\phi} \subset \operatorname{pr}^{-1}(q)$ denote the Lefschetz thimble of $F_{q}$ emanating from the critical point $c_{i}$ whose image under $F_{q}$ is the half-line $\mathbf{u}_{i}-\mathbb{R}_{\geq 0} e^{\mathbf{i} \phi}$; it is given as the stable manifold of the Morse function $\Re\left(e^{-\mathbf{i} \phi} F_{q}\right): \operatorname{pr}^{-1}(q) \rightarrow \mathbb{R}:$

$$
\Gamma_{i}^{\phi}=\left\{x \in \operatorname{pr}^{-1}(q): \lim _{t \rightarrow \infty} \varphi_{t}(x)=c_{i}\right\} \cong \mathbb{R}^{n},
$$

where $\varphi_{t}$ is the upward gradient flow ${ }^{18}$ of $\Re\left(e^{-\mathbf{i} \phi} F_{q}\right)$ with respect to the complete Kähler metric $\frac{\mathbf{i}}{2} \sum_{j=1}^{n} d \log x_{j} \wedge d \overline{\log x_{j}}$ on $\operatorname{pr}^{-1}(q)$. The cycles $\Gamma_{1}^{\phi}, \ldots, \Gamma_{N}^{\phi}$ form a basis of the relative homology $H_{n}\left(\operatorname{pr}^{-1}(q),\left\{\Re\left(e^{-\mathbf{i} \phi} F_{q}\right) \leq-M\right\} ; \mathbb{Z}\right)$ for sufficiently large $M$, see [62, Section 3.3.1] for more details (see also Section 7.4.4).

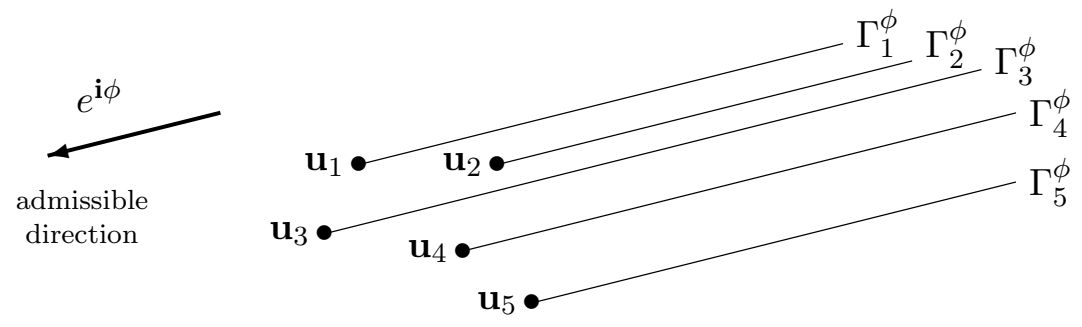

Figure 8. The images of the Lefschetz thimbles $\Gamma_{i}^{\phi}$ by $F_{q}=\left.F\right|_{\mathrm{pr}^{-1}(q)}$.

Remark 6.17. For a non-admissible phase $\phi$, the Lefschetz thimble $\Gamma_{i}^{\phi}$ is not always defined because $\Gamma_{i}^{\phi}$ may hit other critical points $c_{j}$ with $\mathbf{u}_{j} \in \mathbf{u}_{i}-\mathbb{R}_{>0} e^{\mathbf{i} \phi}$. On the other hand, when some of the critical values $\mathbf{u}_{1}, \ldots, \mathbf{u}_{N}$ coalesce at $q_{0} \in \mathcal{M}^{\mathrm{sm}, \mathrm{ss}}$ and $\phi$ is an admissible phase for the critical values of $F_{q_{0}}$, the Lefschetz thimbles $\Gamma_{1}^{\phi}, \ldots, \Gamma_{N}^{\phi}$ are well-defined in a neighbourhood of $q=q_{0}$ despite the possibility that $\phi$ can be non-admissible at a nearby point. This is because different Lefschetz thimbles associated with the same critical value do not intersect each other, and no non-trivial Picard-Lefschetz transformations occur among these thimbles around $q=q_{0}$.

Proposition 6.18. Let $q_{0}$ be a point in $\mathcal{M}^{\mathrm{sm}, \mathrm{ss}}$. Choose an admissible phase $\phi$ for the critical values $\left\{\mathbf{u}_{1,0}, \ldots, \mathbf{u}_{N, 0}\right\}$ of $F_{q_{0}}$. Choose a sufficiently small open neighbourhood $B \subset \mathcal{M}^{\mathrm{sm}, \mathrm{ss}}$ of $q_{0}$ and a sufficiently small number $\epsilon>0$ such that $e^{-\mathbf{i} \phi^{\prime}}\left(\mathbf{u}_{i}-\mathbf{u}_{j}\right) \notin \mathbb{R}$ whenever $q \in B$, $\left|\phi-\phi^{\prime}\right|<\epsilon$ and $\mathbf{u}_{i, 0} \neq \mathbf{u}_{j, 0}$. Let $\pi: \mathcal{M}^{\mathrm{sm}, \mathrm{ss}} \times \widetilde{\mathbb{C}} \rightarrow \mathcal{M}^{\mathrm{sm}, \mathrm{ss}} \times \mathbb{C}$ denote the oriented real blowup along $\mathcal{M}^{\mathrm{sm}, \mathrm{ss}} \times\{0\}$. Define the map

$$
\Phi_{\phi}:\left.\quad \pi^{*} \operatorname{Bri}(F)\right|_{B \times I_{\phi}} \rightarrow \mathcal{A}_{B \times I_{\phi}}^{\oplus N}
$$

by

$$
\Phi_{\phi}(s)(q, z)=\left((-2 \pi z)^{-n / 2} e^{-\mathbf{u}_{i} / z} \int_{\Gamma_{i}^{\phi+\delta}} e^{F_{q} / z} s\right)_{i=1}^{N}, \quad(q, z) \in B \times I_{\phi},
$$

\footnotetext{
${ }^{18}$ The gradient flow of $\Re\left(e^{-\mathbf{i} \phi} F_{q}\right)$ equals the Hamiltonian flow of $\Im\left(e^{-\mathbf{i} \phi} F_{q}\right)$ and thus preserves $\Im\left(e^{-\mathbf{i} \phi} F_{q}\right)$.
} 
where $I_{\phi}=\left\{\left(r, e^{\mathbf{i} \theta}\right):|\theta-\phi|<\frac{\pi}{2}+\epsilon\right\}$ and we choose $\delta \in(-\epsilon, \epsilon)$ depending on the argument of $z$ so that the integral converges. Then $\Phi_{\phi}$ is an isomorphism that identifies the Gauss-Manin connection $\nabla$ with $\bigoplus_{i=1}^{N}\left(d+d\left(\mathbf{u}_{i} / z\right)\right)$ and induces the formal decomposition in Proposition 6.10 (combined with Proposition 6.16) if $B \subset \mathcal{V}^{\mathrm{ss}}$ :

Asym: $\operatorname{Bri}(F) \otimes_{\mathcal{O}_{B \times \mathbb{C}_{z}}^{\text {an }}} \mathcal{O}_{B}^{\text {an }} \llbracket z \rrbracket \cong \mathcal{O}_{B}^{\text {an }} \llbracket z \rrbracket^{\oplus N}$.

Proof. First observe that the oscillatory integral $\Phi_{\phi}(s)$ converges for a suitable choice of $\delta \in$ $(-\epsilon, \epsilon)$, and does not depend on $\delta$ as far as it converges. If $|\arg (z)-\phi|<\frac{\pi}{2}$, we can choose $\delta=0$ because $\Re\left(F_{q} / z\right) \rightarrow-\infty$ in the end of $\Gamma_{i}^{\phi}$; if not we can choose a suitable $\delta$ so that $\Re\left(F_{q} / z\right) \rightarrow-\infty$ in the end of $\Gamma_{i}^{\phi+\delta}$. The fact that $\Phi_{\phi}$ identifies the connections follows from the definition of the Gauss-Manin connection, see [62, equation (54), Lemma 3.15]. Note that the shift of the connection $\nabla_{z \frac{\partial}{\partial z}}$ by $n / 2$ in Remark 4.6 is compensated by the prefactor $(-2 \pi z)^{-n / 2}$. The last statement follows from the fact that $\operatorname{Asym}_{c_{i}}(s)$ gives the asymptotic expansion of the $i$ th component of $\Phi_{\phi}(s)$ along the sector $I_{\phi}$.

Remark 6.19. The choice of an orientation of $\Gamma_{i}^{\phi+\delta}$ and the choice of a branch of $(-2 \pi z)^{-n / 2}$ in (6.13) together give rise to a section of the $\mu_{2}$-local system ori in Proposition 6.10.

\section{Functoriality under toric birational morphisms}

We study the analytic lift of the formal decomposition of the quantum D-modules in Theorem 5.16 in the case where the birational map $\mathfrak{X}_{+} \rightarrow \mathfrak{X}_{-}$extends to a morphism. We show that the analytic lift associated with a certain deformation parameter $\tau_{+}$and a phase is induced by the pull-back between the $K$-groups via the $\widehat{\Gamma}$-integral structure. Moreover, the sectorial decomposition of the quantum D-module of $\mathfrak{X}_{+}$at some $\tau_{+}$corresponds to an Orlov-type semiorthogonal decomposition of the $K$-group. We assume that both $\mathfrak{X}_{+}$and $\mathfrak{X}_{-}$are compact weak-Fano smooth toric DM stacks and restrict ourselves to the non-equivariant quantum D-modules.

\subsection{Notation and assumption}

Consider a discrepant transformation $\mathfrak{X}_{+}-\rightarrow \mathfrak{X}_{-}$arising from a codimension-one wall crossing as in Section 5.1. Let $\boldsymbol{\Sigma}_{ \pm}$be the stacky fan of $\mathfrak{X}_{ \pm}$. We assume that $\mathfrak{X}_{+} \rightarrow \mathfrak{X}_{-}$extends to a birational morphism $\varphi: \mathfrak{X}_{+} \rightarrow \mathfrak{X}_{-}$. In this case, the common blowup $\widehat{\mathfrak{X}}$ in (5.2) is isomorphic to $\mathfrak{X}_{+}$, and $\varphi$ is necessarily a type (II-i) or (III) discrepant transformation in the classification of Remark 5.4, i.e., $\varphi$ is a divisorial contraction or a root construction. We further assume that

- $\mathfrak{X}_{+}$and $\mathfrak{X}_{-}$are compact weak Fano toric stacks; we write $\Delta_{ \pm} \subset \mathbf{N}_{\mathbb{R}}$ for the fan polytopes of $\mathfrak{X}_{ \pm}$; they are convex polytopes containing the origin in their interiors and we have $\Delta_{-} \subset \Delta_{+}$;

- $S_{-}:=S \cap \Delta_{-}=\left\{b \in \mathbf{N}: \bar{b} \in \Delta_{-}\right\}$generates $\mathbf{N}$ over $\mathbb{Z}$.

Here the fan polytope $\Delta_{ \pm}$means the convex hull of ray vectors of the stacky fan $\boldsymbol{\Sigma}_{ \pm}$and $S$ is a finite subset of $\mathbf{N}$ as in Section 3.1 such that both $\boldsymbol{\Sigma}_{+}$and $\boldsymbol{\Sigma}_{-}$are adapted to $S$ in the sense of Definition 3.3. We need these assumptions so that we can apply the results [62] on the $\widehat{\Gamma}$-integral structure for toric stacks, where the same assumptions were made (see Section 3.1.4 ibid).

As in Section 5.1, let $W$ denote the hyperplane wall between the maximal cones $\operatorname{cpl}\left(\boldsymbol{\Sigma}_{+}\right)$and $\operatorname{cpl}\left(\boldsymbol{\Sigma}_{-}\right)$of the secondary fan $\Xi$, and let $\mathbf{w} \in \mathbb{L}$ denote the primitive normal vector of the wall $W$ pointing towards $\operatorname{cpl}\left(\boldsymbol{\Sigma}_{+}\right)$. Set $M_{ \pm}=\left\{b \in S: \pm D_{b} \cdot \mathbf{w}>0\right\}$ as before. When the wall-crossing 

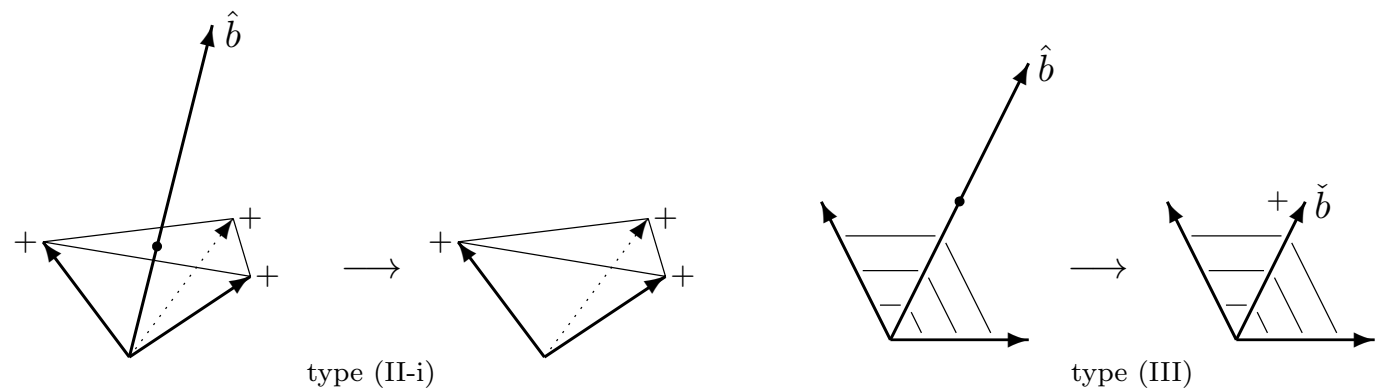

Figure 9. The change of fans associated with $\mathfrak{X}_{+} \rightarrow \mathfrak{X}_{-}$. The sign + means ray vectors from $M_{+} \subset$ $R_{-}=R\left(\boldsymbol{\Sigma}_{-}\right)$and $\hat{b}$ is a positive integral linear combination of $\left\{v: v \in M_{+}\right\}$.

induces a morphism $\mathfrak{X}_{+} \rightarrow \mathfrak{X}_{-}, M_{-}$is a singleton $\{\hat{b}\}$ with $D_{\hat{b}} \cdot \mathbf{w}=-1$ and the corresponding circuit is (see (5.1))

$$
\hat{b}=\sum_{b \in M_{+}} k_{b} b \quad \text { with } k_{b}:=D_{b} \cdot \mathbf{w} .
$$

Assumption 5.2 gives $\sum_{b \in M_{+}} k_{b}>1$. In the type (II-i) case, we have $\sharp M_{+} \geq 2$ and the stacky fan $\boldsymbol{\Sigma}_{+}$is obtained from $\boldsymbol{\Sigma}_{-}$by adding the new ray $\hat{b}$; the cone $\sigma_{M_{+}}=\sum_{b \in M_{+}} \mathbb{R}_{\geq 0} \bar{b}$ of $\boldsymbol{\Sigma}_{-}$is subdivided into cones $\sigma_{M_{+} \cup\{\hat{b}\} \backslash\{v\}}$ with $v \in M_{+}$. In the type (III) case, $M_{+}$is also a singleton $\{\check{b}\}$ and $\boldsymbol{\Sigma}_{+}$is obtained from $\boldsymbol{\Sigma}_{-}$by replacing $\check{b}$ with $\hat{b}=k_{\breve{b}} \breve{b}$ : see Fig. 9 . We write $R_{ \pm}=R\left(\boldsymbol{\Sigma}_{ \pm}\right)$ for the set of rays. Then $R_{+}=R_{-} \sqcup\{\hat{b}\}$ in the type (II-i) case and $R_{+} \sqcup\{\check{b}\}=R_{-} \sqcup\{\hat{b}\}$ in the type (III) case, where $\sqcup$ denotes disjoint union. We also note that $M_{+} \subset R_{-}$. For simplicity of notation, we assume that $S$ is chosen to be a minimal extension of $S_{-}$:

- $S=S_{-} \cup\{\hat{b}\}$.

We do not lose any generality by this assumption: the base $\mathcal{M}$ of the LG model for a larger $S$ always contains the locus corresponding to $S_{-} \cup\{\hat{b}\}$.

The smooth toric DM stacks $\mathfrak{X}_{+}, \mathfrak{X}_{-}$are the GIT quotients of $\mathbb{C}^{S}$. The toric birational morphism $\varphi: \mathfrak{X}_{+} \rightarrow \mathfrak{X}_{-}$is induced by the self-map

$$
\left(z_{\hat{b}},\left(z_{v}\right)_{v \in S \backslash\{\hat{b}\}}\right) \longmapsto\left(1,\left(z_{\hat{b}}^{k_{v}} z_{v}\right)_{v \in S \backslash\{\hat{b}\}}\right)
$$

on $\mathbb{C}^{S} \cong \mathbb{C}^{\{\hat{b}\}} \times \mathbb{C}^{S \backslash\{\hat{b}\}}$, where note that $k_{v} \geq 0$ for $v \neq \hat{b}$. It is easy to check that this sends the stable locus for $\operatorname{cpl}\left(\boldsymbol{\Sigma}_{+}\right)$to the stable locus for $\operatorname{cpl}\left(\boldsymbol{\Sigma}_{-}\right)$. The map $\varphi$ contracts the divisor $\left\{z_{\hat{b}}=0\right\}$ onto the toric substack $Z=\bigcap_{v \in M_{+}}\left\{z_{v}=0\right\}$. In the type (II-i) case, $\varphi$ is a weighted blowup along the codimension $\geq 2$ substack $Z$; in the type (III) case where $M_{+}$is a singleton $\{\check{b}\}$, $\varphi$ exhibits $\mathfrak{X}_{+}$as a root stack of $\mathfrak{X}_{-}$with respect to the divisor $Z=\left\{z_{\breve{b}}=0\right\}$.

We write $(\operatorname{pr}: \mathcal{Y} \rightarrow \mathcal{M}, F)$, (pr: $\left.\mathcal{Y}^{\mathrm{sm}} \rightarrow \mathcal{M}^{\mathrm{sm}}, F\right)$ for the LG models associated with $S$ and $S_{-}=S \cap \Delta_{-}$respectively (see Definition 3.6). These two LG models are related by the pull-back (Lemma 6.12):

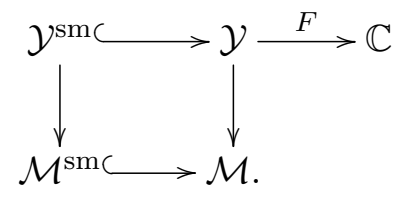


We write $\operatorname{Bri}(F), \operatorname{Bri}^{\mathrm{sm}}(F)$ for the non-equivariant Brieskorn modules (Definition 4.1) associated with the LG models $(\mathrm{pr}: \mathcal{Y} \rightarrow \mathcal{M}, F)$, ( $\left.\mathrm{pr}: \mathcal{Y}^{\mathrm{sm}} \rightarrow \mathcal{M}^{\mathrm{sm}}, F\right)$ respectively. On the affine chart associated with $\boldsymbol{\Sigma}_{-}, \mathcal{M}^{\mathrm{sm}}$ is cut out from $\mathcal{M}$ by the equation $t_{\hat{b}}=0$, where $t_{\hat{b}}=q^{\delta_{\hat{b}}}$ is the coordinate introduced in Section 3.5. We have $t_{\hat{b}}=q^{-\mathbf{w}}$ since $\delta_{\hat{b}}^{\boldsymbol{\Sigma}_{-}}=e_{\hat{b}}-\Psi_{-}(\hat{b})=e_{\hat{b}}-\sum_{b \in M_{+}} k_{b} e_{b}=$ $-\mathbf{w} \in \mathbb{L}$, where $\Psi_{-}=\Psi^{\boldsymbol{\Sigma}_{-}}$(see Notation 3.10).

In this section, we fix an isomorphism $\mathbf{N} \cong \mathbb{Z}^{n} \times \mathbf{N}_{\text {tor }}$ and a splitting $\varsigma: \mathbf{N} \rightarrow \mathbb{O}^{\boldsymbol{\Sigma}_{-}}$of the refined fan sequence (3.12) for $\boldsymbol{\Sigma}_{-}$and use the associated co-ordinates $x_{1}, \ldots, x_{n}$ along the fibres of the LG model as introduced in Section 3.5.

Caution 7.1. Under our assumption $S=S_{-} \cup\{\hat{b}\}, \mathcal{M}$ is the small quantum cohomology locus (see Section 6.3 .2 ) of $\mathfrak{X}_{+}$and $\mathcal{M}^{\mathrm{sm}}$ is the small quantum cohomology locus of $\mathfrak{X}_{-}$.

\subsection{Critical points along a curve}

Consider the 1-dimensional toric substack $\mathcal{C}$ of $\mathcal{M}$ corresponding to $W \cap \operatorname{cpl}\left(\boldsymbol{\Sigma}_{+}\right)=W \cap \operatorname{cpl}\left(\boldsymbol{\Sigma}_{-}\right)$. Recall that uniformizing affine charts of $\mathcal{C}$ and $\mathrm{pr}^{-1}(\mathcal{C})$ have been described explicitly in Section 5.2: $q^{-\mathbf{w} / e}=t_{\hat{b}}^{1 / e}$ gives a rational co-ordinate of $\mathcal{C} \cap \mathcal{M}_{\boldsymbol{\Sigma}_{-}}$, where $e=e_{-} \in \mathbb{N}$ is the smallest common denominator of $\left\{c \in \mathbb{Q}: c \mathbf{w} \in \mathbf{\Lambda}\left(\boldsymbol{\Sigma}_{-}\right)\right\}$, and $w_{v}=w_{v}^{-}=u^{\left(\Psi_{-}(v), v\right)}$ with $v \in \mathbf{N}$ generate the co-ordinate ring $A=\bigoplus_{v \in \mathbf{N}} \mathbb{C}\left[t_{\hat{b}}^{1 / e}\right] w_{v}$ of $\operatorname{pr}^{-1}(\mathcal{C}) \cap \mathcal{Y}_{\boldsymbol{\Sigma}_{-}}$(see Lemma 5.8). Recall also that $F=\sum_{b \in R_{+} \cup R_{-}} u_{b}$ on $\operatorname{pr}^{-1}(\mathcal{C})$. We study the family of relative critical points

$$
x_{1} \frac{\partial F}{\partial x_{1}}=\cdots=x_{n} \frac{\partial F}{\partial x_{n}}=0
$$

over the curve $\mathcal{C}$.

Proposition 7.2. A relative critical point of the $L G$ potential $F$ over $\mathcal{C}$ is given by an assignment of a complex number $w_{v}$ to every $v \in \mathbf{N}$ satisfying $w_{0}=1$,

$$
w_{v_{1}} w_{v_{2}}=w_{v_{1}+v_{2}} \quad \text { if } \overline{v_{1}}, \overline{v_{2}} \in \sigma_{M_{+}} \quad \text { and } \quad w_{v}= \begin{cases}0, & \text { if } \bar{v} \notin \sigma_{M_{+}}, \\ k_{v} \gamma, & \text { if } v \in M_{+},\end{cases}
$$

where $k_{v}=D_{v} \cdot \mathbf{w}$ for $v \in S$ and

$$
\gamma=0 \quad \text { or } \quad \gamma=\left(-\frac{1}{K t_{\hat{b}}}\right)^{1 / J} \quad \text { when } t_{\hat{b}} \neq 0
$$

with $J:=\left(\sum_{b \in M_{+}} k_{b}\right)-1>0$ and $K:=\prod_{b \in M_{+}} k_{b}^{k_{b}}$. The corresponding critical value of $F$ is given by $J \gamma$.

Proof. Take a relative critical point and let $w_{v}$ denote the value of the function $w_{v}$ at that point. We have $w_{0}=1$. The equation (7.2) for relative critical points reads

$$
\sum_{b \in R_{+} \cup R_{-}} u_{b} \bar{b}=0 \quad \text { in } \mathbf{N}_{\mathbb{R}} .
$$

Note that $R_{+} \cup R_{-}=R_{-} \sqcup\{\hat{b}\}, u_{b}=w_{b}$ for $b \in R_{-}$and $u_{\hat{b}}=t_{\hat{b}} w_{\hat{b}}$. By Lemma 5.8, we have

$$
w_{v} \cdot w_{v^{\prime}}= \begin{cases}w_{v+v^{\prime}}, & \text { if } \bar{v} \text { and } \overline{v^{\prime}} \text { belong to a common cone of } \Sigma_{-}=\Sigma_{0}, \\ 0, & \text { otherwise }\end{cases}
$$


where note that $\Sigma_{0}$ in Lemma 5.8 coincides with the underlying fan $\Sigma_{-}$of $\boldsymbol{\Sigma}_{-}$in the current setting. Therefore there exists a cone $\sigma \in \Sigma_{-}$such that $\left\{\bar{v}: w_{v} \neq 0\right\}=\mathbf{N} \cap \sigma$. Then equation (7.3) implies that $\left(R_{-} \cup\{\hat{b}\}\right) \cap \sigma$ is linearly dependent. Therefore we have either $\left(R_{-} \cup\{\hat{b}\}\right) \cap \sigma=\varnothing$ or $\bar{b} \in \sigma$. In the former case, we have $\sigma=0$; this corresponds to the case where $\gamma=0$ in the proposition. In the latter case, we have $\sigma \supset \sigma_{M_{+}}$; the linear relation $\hat{b}=\sum_{b \in M_{+}} k_{b} b$ together with (7.3) implies

$$
\left\{\begin{array}{ll}
w_{b}+k_{b} t_{\hat{b}} w_{\hat{b}}=0 & \text { for } b \in M_{+}, \\
w_{b}=0 & \text { for } b \in\left(R_{-} \backslash M_{+}\right) \cap \sigma,
\end{array} \quad \text { and } \quad w_{\hat{b}}=\prod_{b \in M_{+}} w_{b}^{k_{b}} .\right.
$$

Since $w_{b} \neq 0$ for $\bar{b} \in \sigma$, we have $\left(R_{-} \backslash M_{+}\right) \cap \sigma=\varnothing$ and thus $\sigma=\sigma_{M_{+}}$. Setting $\gamma=w_{b} / k_{b}=-t_{\hat{b}} w_{\hat{b}}$ (with $b \in M_{+}$), we find that $\gamma$ satisfies

$$
-\gamma / t_{\hat{b}}=\prod_{b \in M_{+}}\left(k_{b} \gamma\right)^{k_{b}} \Longleftrightarrow-1 /\left(K t_{\hat{b}}\right)=\gamma^{J}
$$

This proves the proposition.

Remark 7.3. Proposition 7.2 implies that the critical values of $F$ along $\mathcal{C}$ do not belong to $\mathbb{R}_{>0}$ when $t_{\hat{b}}>0$. This phenomenon is closely related to the Conjecture $\mathcal{O}$ [44, Conjecture 3.1.2].

\subsection{Identifying $\mathcal{O}$}

Recall from Section 2.4 that the $\widehat{\Gamma}$-integral structure identifies the $K$-group of a smooth DM stack with a lattice of flat sections of the quantum D-module. In this section, we introduce a 'positive real' Lefschetz thimble $\Gamma_{\mathbb{R}}$ defined along a 'positive real' locus $\mathcal{M}_{\mathbb{R}}$ (or $\mathcal{M}_{\mathbb{R}}^{\text {sm }}$ ). The flat section $\mathfrak{s}_{\mathcal{O}}$ given by the structure sheaf $\mathcal{O}$ corresponds to $\Gamma_{\mathbb{R}}$ under mirror symmetry, and thus spans a component of the analytic lift of the formal decomposition (as discussed in Propositions 6.5 and 6.18) associated with the so-called conifold point. The content in this section is essentially an adaptation of the main result of [62] to the current setting.

\subsubsection{The structure sheaf of $\mathfrak{X}_{+}$}

By definition (see Section 3.4), $\mathcal{M}$ and $\mathcal{Y}$ contain open dense tori $\mathcal{M}^{\times}=\mathbb{L}^{\star} \otimes \mathbb{C}^{\times}, \mathcal{Y}^{\times}=\left(\mathbb{C}^{\times}\right)^{S}$ respectively. We define the positive real loci $\mathcal{M}_{\mathbb{R}} \subset \mathcal{M}^{\times}, \mathcal{Y}_{\mathbb{R}} \subset \mathcal{Y}^{\times}$to be

$$
\mathcal{M}_{\mathbb{R}}:=\mathbb{L}^{\star} \otimes \mathbb{R}_{>0}, \quad \mathcal{Y}_{\mathbb{R}}:=\left(\mathbb{R}_{>0}\right)^{S} .
$$

We write $\mathcal{Y}_{q}$ for the fibre of pr: $\mathcal{Y} \rightarrow \mathcal{M}$ at $q \in \mathcal{M}$; and $\Gamma_{\mathbb{R}}=\Gamma_{\mathbb{R}}(q)$ for the fibre of $\mathcal{Y}_{\mathbb{R}} \rightarrow \mathcal{M}_{\mathbb{R}}$ at $q \in \mathcal{M}_{\mathbb{R}}$ :

$$
\Gamma_{\mathbb{R}}(q)=\mathcal{Y}_{q} \cap \mathcal{Y}_{\mathbb{R}} \cong \operatorname{Hom}\left(\mathbf{N}, \mathbb{R}_{>0}\right) \cong\left(\mathbb{R}_{>0}\right)^{n}
$$

Consider the restriction of $F_{q}=\left.F\right|_{\mathcal{Y}_{q}}$ to the real positive locus $\Gamma_{\mathbb{R}}$. Then

- it is a strictly convex function since the Hessian $\frac{\partial^{2} F_{q}}{\partial \log x_{i} \partial \log x_{j}}=\sum_{b \in S} u_{b} b_{i} b_{j}$ is positive definite on $\Gamma_{\mathbb{R}}$ (where $b_{i} \in \mathbb{Z}$ is the $i$ th entry of $\bar{b} \in \overline{\mathbf{N}} \cong \mathbb{Z}^{n}$ );

- it is proper and bounded from below since 0 is in the interior of the convex hull $\Delta_{+}$of $\{\bar{b}: b \in S\}$. 
Therefore, $\left.F_{q}\right|_{\Gamma_{\mathbb{R}}}$ attains a global minimum at a unique critical point $\operatorname{cr}_{\mathbb{R}}=\operatorname{cr}_{\mathbb{R}}(q) \in \Gamma_{\mathbb{R}}(q)$; the point $\mathrm{cr}_{\mathbb{R}}$ is called the conifold point $[43,44]$. Since $\Gamma_{\mathbb{R}}$ is preserved by the gradient flow of $\Re\left(F_{q}\right)$ (with respect to the Kähler metric $\frac{\mathrm{i}}{2} \sum_{i=1}^{n} d \log x_{i} \wedge d \overline{\log x_{i}}$ ) on $\mathcal{Y}_{q}=\operatorname{pr}^{-1}(q)$, we have the following:

Lemma 7.4. The positive real locus $\Gamma_{\mathbb{R}}$ of $\mathcal{Y}_{q}$ is the Lefschetz thimble (6.12) of $F_{q}$ associated with the conifold point $\mathrm{cr}_{\mathbb{R}}$ and the phase $\phi=\pi$.

By Proposition 6.16, there exist an analytic neighbourhood $\mathcal{V}_{+}$of $0_{+}:=0_{\boldsymbol{\Sigma}_{+}} \in \mathcal{M}$, a mirror map $\operatorname{mir}_{+}: \mathcal{V}_{+} \rightarrow\left[\mathcal{M}_{\mathrm{A}}\left(\mathfrak{X}_{+}\right) / \operatorname{Pic}^{\text {st }}\left(\mathfrak{X}_{+}\right)\right]$and an isomorphism

$$
\operatorname{Mir}_{+}:\left.\operatorname{Bri}(F)\right|_{\mathcal{V}_{+}} \cong \operatorname{mir}_{+}^{*} \mathrm{QDM}^{\mathrm{an}}\left(\mathfrak{X}_{+}\right),
$$

where $\mathrm{QDM}^{\mathrm{an}}\left(\mathfrak{X}_{+}\right)$denotes the analytic quantum D-module as in (6.1). The following result says that $\Gamma_{\mathbb{R}}(q)$ corresponds to the flat section $\mathfrak{s}_{\mathcal{O}}$ under mirror symmetry:

Theorem 7.5 ([62, Theorems 4.11 and 4.14, Section 4.3.1]). Let $P(\alpha, \beta)=(\alpha(-z), \beta(z))$ denote the pairing (2.9) of the quantum D-module induced by the orbifold Poincaré pairing. We write $\Omega=\Omega_{q, z}$ for a local section of $\operatorname{Bri}(F)$.

(1) For $q \in \mathcal{V}_{+} \cap \mathcal{M}_{\mathbb{R}}$, we have an isomorphism

$$
H_{n}\left(\mathcal{Y}_{q},\left\{\Re\left(-F_{q}\right) \ll 0\right\} ; \mathbb{Z}\right) \cong K\left(\mathfrak{X}_{+}\right), \quad \Gamma \mapsto V(\Gamma),
$$

which varies locally constantly in $q$ such that

$$
(2 \pi z)^{-n / 2} \int_{\Gamma} e^{-F_{q} / z} \Omega_{q,-z}=P\left(\operatorname{Mir}_{+}(\Omega), \mathfrak{s}_{V(\Gamma)}\left(\operatorname{mir}_{+}(q), z\right)\right)
$$

for $\Gamma \in H_{n}\left(\mathcal{Y}_{q},\left\{\Re\left(-F_{q}\right) \ll 0\right\} ; \mathbb{Z}\right), \Omega \in \operatorname{Bri}(F)$ and $z>0$, where $\mathfrak{s}_{V}(\tau, z)$ is the flat section of the $\widehat{\Gamma}$-integral structure (see Definition 2.7).

(2) In $(1), V\left(\Gamma_{\mathbb{R}}(q)\right)$ is given by the structure sheaf $\mathcal{O}$ of $\mathfrak{X}_{+}$.

In other words, the integral structure of the Brieskorn module $\operatorname{Bri}(F)$ dual to the lattice

$$
H_{n}\left(\mathcal{Y}_{q},\left\{\Re\left(F_{q} / z\right) \ll 0\right\} ; \mathbb{Z}\right)
$$

corresponds to the $\widehat{\Gamma}$-integral structure of the quantum D-module under the mirror isomorphism.

Remark 7.6 ([62, Section 4.3]). Since the mirror isomorphism $\mathrm{Mir}_{+}$preserves the pairing, the map $\Gamma \mapsto V(\Gamma)$ above satisfies

$$
(-1)^{n(n-1) / 2} \#\left(e^{-\pi \mathbf{i}} \Gamma_{1} \cdot \Gamma_{2}\right)=\chi\left(V\left(\Gamma_{1}\right), V\left(\Gamma_{2}\right)\right)
$$

for $\Gamma_{1}, \Gamma_{2} \in H_{n}\left(\mathcal{Y}_{q},\left\{\Re\left(-F_{q}\right) \ll 0\right\} ; \mathbb{Z}\right)$, where $\chi\left(V_{1}, V_{2}\right)$ is the Euler pairing,

$$
e^{-\pi \mathbf{i}} \Gamma_{1} \in H_{n}\left(\mathcal{Y}_{q_{*}},\left\{\Re\left(F_{q_{*}}\right) \ll 0\right\} ; \mathbb{Z}\right)
$$

is the parallel translate of $\Gamma_{1}$ in the local system

$$
\bigcup_{\theta \in[0, \pi]} H_{n}\left(\mathcal{Y}_{q_{*}},\left\{\Re\left(-e^{\mathrm{i} \theta} F_{q_{*}}\right) \ll 0\right\} ; \mathbb{Z}\right)
$$

from $\theta=0$ to $\theta=\pi$, and \# $\left(e^{-\pi \mathbf{i}} \Gamma_{1} \cdot \Gamma_{2}\right)$ denotes the algebraic intersection number. Here we use the fact that the higher residue pairing corresponds to the intersection pairing on relative homology (see [62, Sections 3.3.1-3.3.2]); note however that the sign factor $(-1)^{n(n-1) / 2}$ was missing in [62], see [64, footnote (16)] for the correction. 
Remark 7.7 (cf. Remark 2.8). We need to specify a branch of the multi-valued section $\mathfrak{s}_{V}$ in the above theorem. We have a standard choice for the branch of $\mathfrak{s}_{V}\left(\operatorname{mir}_{+}(q), z\right)$ with $V \in K\left(\mathfrak{X}_{+}\right)$ when $q \in \mathcal{M}_{\mathbb{R}}$, and the above theorem holds for this choice. By the argument preceding [62, Proposition 4.8], the fundamental solution $L(\operatorname{mir}(q), z)$ can be obtained from the $I$-function via the Birkhoff factorization; the $I$-function has a standard determination on the positive real locus (by requiring $\log q_{a} \in \mathbb{R}$ in the formula [62, (59)] of the $I$-function). We also have a standard determination of $z^{-\mu} z^{c_{1}(\mathfrak{X})}$ given by $\log z \in \mathbb{R}$ for $z>0$. Hence we obtain a standard identification of the space of flat sections of $\operatorname{Bri}(F)$ over $(q, z) \in\left(\mathcal{M}_{\mathbb{R}} \cap \mathcal{V}_{+}\right) \times \mathbb{R}_{>0}$ with the $K$-group $K\left(\mathfrak{X}_{+}\right) \otimes \mathbb{C}$.

Introduce the following subsets of $\mathcal{V}_{+} \subset \mathcal{M}$ :

$$
\begin{aligned}
& \mathcal{V}_{+}^{\text {ss }}:=\left\{q \in \mathcal{V}_{+} \cap \mathcal{M}^{\times}: F_{q}=\left.F\right|_{\mathcal{Y}_{q}} \text { has only non-degenerate critical points }\right\} \\
& \mathcal{V}_{+, \mathbb{R}}^{\text {ss }}:=\mathcal{V}_{+}^{\text {ss }} \cap \mathcal{M}_{\mathbb{R}} .
\end{aligned}
$$

Note that $\mathcal{V}_{+}^{\text {ss }}$ is the intersection of $\mathcal{V}_{+}$and a non-empty Zariski-open subset of $\mathcal{M}$ by the discussion in Section 6.3.3; hence $\mathcal{V}_{+}^{\text {ss }}$ is open dense in $\mathcal{V}_{+}$, and $\mathcal{V}_{+, \mathbb{R}}^{\text {ss }}$ is open dense in $\mathcal{V}_{+} \cap \mathcal{M}_{\mathbb{R}}$.

Choose $q_{0} \in \mathcal{V}_{+, \mathbb{R}}^{\text {ss }}$ and let $\mathrm{cr}_{1}(q), \ldots, \mathrm{cr}_{N_{+}}(q)$ denote all branches of critical points of $F_{q}$ near $q=q_{0}$, where $N_{+}=\operatorname{dim} H_{\mathrm{CR}}^{*}\left(\mathfrak{X}_{+}\right)$. We may assume that $\operatorname{cr}_{1}\left(q_{0}\right)$ is the conifold point $\mathrm{cr}_{\mathbb{R}}\left(q_{0}\right)$. Let $\mathbf{u}_{i}(q)=F_{q}\left(\operatorname{cr}_{i}(q)\right)$ be the corresponding critical value. Suppose that $\phi \in \mathbb{R}$ is an admissible phase for $\left\{\mathbf{u}_{1}\left(q_{0}\right), \ldots, \mathbf{u}_{N_{+}}\left(q_{0}\right)\right\}$. By Proposition 6.18 , there exist an open neighbourhood $B$ of $q_{0}$ in $\mathcal{V}_{+}^{\text {ss }}$, a sector $I_{\phi}=\left\{\left(r, e^{\mathbf{i} \theta}\right) \in \widetilde{\mathbb{C}}:|\theta-\phi|<\frac{\pi}{2}+\epsilon\right\}$ (with small $\epsilon>0$ ) and an isomorphism (analytic lift)

$$
\Phi_{\phi}^{+}:\left.\pi^{*} \operatorname{Bri}(F)\right|_{B \times I_{\phi}} \cong \bigoplus_{i=1}^{N_{+}}\left(\mathcal{A}_{B \times I_{\phi}}, d+d\left(\mathbf{u}_{i}(q) / z\right)\right)
$$

that induces the formal decomposition Asym: $\operatorname{Bri}(F) \otimes_{\mathcal{O}_{B \times \mathbb{C}_{z}}^{\text {an }}} \mathcal{O}_{B}^{\text {an }} \llbracket z \rrbracket \cong \mathcal{O}_{B}^{\text {an }} \llbracket z \rrbracket^{\oplus N_{+}}$, where $\pi: \mathcal{V}_{+}^{\text {ss }} \times \widetilde{\mathbb{C}} \rightarrow \mathcal{V}_{+}^{\text {ss }} \times \mathbb{C}$ is the oriented real blow-up. Composing this with the mirror isomorphism, we also get the analytic lift of the formal decomposition of the quantum D-module (cf. Proposition 6.5):

$$
\widetilde{\Phi}_{\phi}^{+}:\left.\pi^{*} \operatorname{mir}_{+}^{*} \operatorname{QDM}^{\mathrm{an}}\left(\mathfrak{X}_{+}\right)\right|_{B \times I_{\phi}} \cong \bigoplus_{i=1}^{N_{+}}\left(\mathcal{A}_{B \times I_{\phi}}, d+d\left(\mathbf{u}_{i}(q) / z\right)\right),
$$

where $\widetilde{\Phi}_{\phi}^{+}=\Phi_{\phi}^{+} \circ \operatorname{Mir}_{+}^{-1}$.

Proposition 7.8. For $q_{0} \in \mathcal{V}_{+, \mathbb{R}}^{\mathrm{ss}}$, there exists $\alpha_{0} \in(0, \pi / 2)$ such that the following holds. For every admissible phase $\phi \in\left(-\alpha_{0}, \alpha_{0}\right)$ for $\left\{\mathbf{u}_{1}\left(q_{0}\right), \ldots, \mathbf{u}_{N_{+}}\left(q_{0}\right)\right\}$, there exists a basis $\left\{V_{i}\right\}_{i=1}^{N_{+}}$of $K\left(\mathfrak{X}_{+}\right)$with $V_{1}$ being the structure sheaf $\mathcal{O}$ of $\mathfrak{X}_{+}$, such that the corresponding flat section $s_{i}=$ $\mathfrak{s}_{V_{i}}\left(\operatorname{mir}_{+}(q), z\right)$ satisfies $\widetilde{\Phi}_{\phi}^{+}\left(e^{\mathbf{u}_{i}(q) / z} s_{i}\right)=e_{i}$, where $e_{i}$ denotes the ith standard basis of $\mathcal{A}_{B \times I_{\phi}}^{\oplus N_{+}}$.

Proof. Let $\Gamma_{i}^{\phi}(q)$ denote the Lefschetz thimble (6.12) of $F_{q}$ associated with the critical point $\operatorname{cr}_{i}(q)$ and phase $\phi$. By Lemma 7.4, $\Gamma_{1}^{\pi}\left(q_{0}\right)=\Gamma_{\mathbb{R}}\left(q_{0}\right)$. Since there are no critical points of $F_{q_{0}}$ on $\Gamma_{\mathbb{R}}\left(q_{0}\right)$ other than the conifold point $\mathrm{cr}_{\mathbb{R}}\left(q_{0}\right), \Gamma_{1}^{\pi+\phi}(q)$ varies continuously in a neighbourhood of $(q, \phi)=\left(q_{0}, 0\right)$. Let $\alpha_{0} \in(0, \pi / 2)$ be such that $\Gamma_{1}^{\pi+\phi}(q)$ depends continuously on $(q, \phi)$ as $(q, \phi)$ varies in a neighbourhood of $\left\{q_{0}\right\} \times\left(-\alpha_{0}, \alpha_{0}\right)$ in $\mathcal{V}_{+}^{\mathrm{ss}} \times \mathbb{R}$.

Choose an admissible phase $\phi \in\left(-\alpha_{0}, \alpha_{0}\right)$ for $\left\{\mathbf{u}_{1}\left(q_{0}\right), \ldots, \mathbf{u}_{N_{+}}\left(q_{0}\right)\right\}$ and let $\Phi_{\phi}^{+}, \widetilde{\Phi}_{\phi}^{+}$be the associated analytic lifts over a neighbourhood $B \times I_{\phi}$ of $\left(q_{0},\left(0, e^{\mathbf{i} \phi}\right)\right)$ as above. Let $\varphi_{i}$ denote a section of $\pi^{*}$ mir $\left._{+}^{*} \operatorname{QDM}^{\text {an }}\left(\mathfrak{X}_{+}\right)\right|_{B \times I_{\phi}}$ such that $\widetilde{\Phi}_{\phi}^{+}\left(\varphi_{i}\right)=e_{i}$. Take a local section $\Omega=\Omega_{q, z}$ of 
$\operatorname{Bri}(F)$ near $q=q_{0}$. By the definition (6.13) of $\Phi_{\phi}^{+}$, for $q \in B$ and $z \in \mathbb{C}^{\times}$with $|\arg (z)-\phi|<\pi / 2$, we have ${ }^{19}$

$$
\begin{aligned}
(2 \pi z)^{-n / 2} e^{\mathbf{u}_{i}(q) / z} \int_{\Gamma_{i}^{\phi+\pi}(q)} e^{-F_{q} / z} \Omega_{q,-z} & =\Phi_{\phi+\pi}^{i}(\Omega)(q,-z) \\
& =P_{\text {std }}\left(\Phi_{\phi+\pi}(\Omega), e_{i}\right)(q, z) \\
& =P_{\text {std }}\left(\widetilde{\Phi}_{\phi+\pi}\left(\operatorname{Mir}_{+}(\Omega)\right), \widetilde{\Phi}_{\phi}\left(\varphi_{i}\right)\right)(q, z) \\
& =P\left(\operatorname{Mir}_{+}(\Omega), \varphi_{i}\right)(q, z),
\end{aligned}
$$

where $P_{\text {std }}$ is the diagonal pairing as in Proposition 6.10 and we used Remark 6.7 in the last step. By Theorem 7.5(1), the left-hand side of the above equation equals

$$
e^{\mathbf{u}_{i}(q) / z} P\left(\operatorname{Mir}_{+}(\Omega), \mathfrak{s}_{V_{i}}\left(\operatorname{mir}_{+}(q), z\right)\right) \quad \text { with } V_{i}=V\left(\Gamma_{i}^{\phi+\pi}(q)\right) .
$$

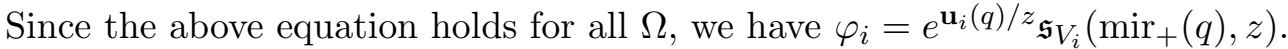

We now set $i=1$. By the choice of $\alpha_{0}$ and $\phi$, the cycle $\Gamma_{1}^{\phi+\pi}\left(q_{0}\right)$ (and hence $\Gamma_{1}^{\phi+\pi}(q)$ with $q \in B)$ is a continuous deformation of $\Gamma_{1}^{\pi}\left(q_{0}\right)=\Gamma_{\mathbb{R}}\left(q_{0}\right)$. Thus we get $V_{1}=V_{1}\left(\Gamma_{\mathbb{R}}\left(q_{0}\right)\right)=\mathcal{O}$ by Theorem 7.5(2).

Remark 7.9. Recall from Propositions 6.1 and 6.10 that the formal decomposition and its analytic lift $\Phi_{\phi}^{+}$are ambiguous up to multiplication by $\operatorname{diag}( \pm 1, \ldots, \pm 1)$, and this ambiguity is fixed once we give a local trivialization of the $\mu_{2}$-local system ori. We note that there is a standard trivialization of ori at the conifold point $\mathrm{cr}_{\mathbb{R}}$ since the Hessian of $F_{q}$ at $\mathrm{cr}_{\mathbb{R}}$ is positivedefinite; hence the component of the analytic lift $\Phi_{\phi}^{+}$corresponding to $\operatorname{cr}_{\mathbb{R}}(q)$ (which is the first component) is unambiguous.

Remark 7.10. Proposition 7.8 says that $\mathfrak{s}_{\mathcal{O}}$ is characterized by the exponential asymptotics $\mathfrak{s}_{\mathcal{O}} \sim e^{-\mathbf{u}_{1}(q) / z} \Psi_{\mathbb{R}}(q)$ as $z \rightarrow 0$ along the sector $\arg z \in\left(-\frac{\pi}{2}-\alpha_{0}, \frac{\pi}{2}+\alpha_{0}\right)$ when $q$ lies in a neighbourhood of the positive real locus, where $\Psi_{\mathbb{R}}(q)$ is the normalized idempotent (see Remark 6.3) corresponding to the conifold point $\mathrm{cr}_{\mathbb{R}}(q)$. In terms of the marked reflection system in Section 6.2 , it also says that $\widehat{\Gamma}_{\mathfrak{X}_{+}} \cup(2 \pi \mathbf{i})^{\operatorname{deg}_{0} / 2} \operatorname{inv}^{*} \widetilde{\operatorname{ch}}\left(V_{i}^{+}\right), 1 \leq i \leq N_{+}$give the asymptotic basis at $q_{0}$ and $\phi$.

Remark 7.11. At various places in Section 7, we work around for the fact that $u_{1}(q)+\mathbb{R}_{\geq 0}$ may contain other critical values; the argument would become much simpler if otherwise.

\subsubsection{The structure sheaf of $\mathfrak{X}_{-}$}

We repeat the same discussion for $\mathfrak{X}_{-}$. The difference is that we consider the analytic lift over a region $\mathcal{V}_{-}$which protrudes from the small quantum cohomology locus $\mathcal{M}^{\text {sm }}$ of $\mathfrak{X}_{-}$.

Let $\mathcal{M}^{\mathrm{sm}, \times}:=\mathbb{L}^{\prime \star} \otimes \mathbb{C}^{\times}, \mathcal{Y}^{\mathrm{sm}, \times}:=\left(\mathbb{C}^{\times}\right)^{S_{-}}$denote the open dense tori in $\mathcal{M}^{\mathrm{sm}}$ and $\mathcal{Y}^{\mathrm{sm}}$ respectively and set:

$$
\begin{aligned}
& \mathcal{M}_{\mathbb{R}}^{\mathrm{sm}}:=\mathbb{L}^{\prime \star} \otimes \mathbb{R}_{>0} \subset \mathcal{M}^{\mathrm{sm}, \times}, \\
& \mathcal{Y}_{\mathbb{R}}^{\mathrm{sm}}:=\left(\mathbb{R}_{>0}\right)^{S_{-}} \subset \mathcal{Y}^{\mathrm{sm}, \times}, \\
& \Gamma_{\mathbb{R}}=\Gamma_{\mathbb{R}}(q):=\text { the fibre of } \mathcal{Y}_{\mathbb{R}}^{\mathrm{sm}} \rightarrow \mathcal{M}_{\mathbb{R}}^{\mathrm{sm}} \text { at } q \in \mathcal{M}_{\mathbb{R}}^{\mathrm{sm}} \text { (as defined before), }
\end{aligned}
$$

where $\mathbb{L}^{\prime}=\operatorname{Ker}\left(\mathbb{Z}^{S_{-}} \rightarrow \mathbf{N}\right)$ is the lattice as in Section 6.3.3. For $q \in \mathcal{M}_{\mathbb{R}}^{\text {sm }}, \Gamma_{\mathbb{R}}(q)$ is the Lefschetz thimble of $F_{q}$ associated with the conifold point $\operatorname{cr}_{\mathbb{R}}(q)$ and the phase $\pi$ as in Lemma 7.4. The

\footnotetext{
${ }^{19}$ Recall from Proposition 6.18 that $B$ is chosen sufficiently small so that $\Gamma_{i}^{\phi+\pi}(q)$ deforms continuously as $q$ varies in $B$.
} 
analytified Brieskorn module $\overline{\operatorname{Bri}^{\mathrm{an}}}(F)_{\boldsymbol{\Sigma}}$ is defined over an analytic open neighbourhood $\mathcal{V}_{-}$ of $0_{-}:=0_{\boldsymbol{\Sigma}_{-}}$in $\mathcal{M}$ (see Definition 4.21). By the non-equivariant limit of Theorem 4.34, by shrinking $\mathcal{V}_{-}$if necessary, we have a mirror map $\operatorname{mir}_{-}: \mathcal{V}_{-} \rightarrow\left[\mathcal{M}_{\mathrm{A}}\left(\mathfrak{X}_{-}\right) / \mathrm{Pic}^{\text {st }}\left(\mathfrak{X}_{-}\right)\right]$and a mirror isomorphism

$$
\operatorname{Mir}_{-}: \overline{\operatorname{Bri}^{\mathrm{an}}}(F)_{\Sigma} \cong \operatorname{mir}_{-}^{*} \overline{\mathrm{QDM}^{\mathrm{an}}}\left(\mathfrak{X}_{-}\right) .
$$

By Proposition 6.16, by shrinking $\mathcal{V}_{-}$further if necessary, this mirror isomorphism can be lifted to a fully analytic mirror isomorphism over $\mathcal{V}_{-}^{\mathrm{sm}}=\mathcal{V}_{-} \cap \mathcal{M}^{\mathrm{sm}}$

$$
\operatorname{Mir}_{-}:\left.\operatorname{Bri}^{\mathrm{sm}}(F)\right|_{\mathcal{V}_{-}^{\mathrm{sm}}} \cong \operatorname{mir}_{-}^{*} \mathrm{QDM}^{\mathrm{an}}\left(\mathfrak{X}_{-}\right) \text {. }
$$

Theorem 7.12 ([62, Theorems 4.11 and 4.14, Section 4.3.1]). The conclusions of Theorem 7.5 hold true even when $\mathfrak{X}_{+}, \mathcal{V}_{+}, \mathrm{Mir}_{+}, \operatorname{mir}_{+}, \mathcal{M}_{\mathbb{R}}$ there are replaced with $\mathfrak{X}_{-}, \mathcal{V}_{-}$, Mir $\mathrm{r}_{-}$, mir $\mathcal{M}_{\mathbb{R}}^{\mathrm{sm}}$ respectively.

By the construction of $\overline{\operatorname{Bri}^{a n}}(F)_{\Sigma_{-}}$in Section 4.3, we have an open neighbourhood $\mathcal{B}_{-}$of $\tilde{0}_{-}:=\tilde{0}_{\Sigma_{-}} \in \mathcal{Y}$ such that the family

$$
\mathrm{Cr}_{-}:=\mathcal{B}_{-} \cap \widetilde{\mathrm{pr}}^{-1}\left(\mathcal{V}_{-} \times\{0\}\right) \stackrel{\mathrm{pr}}{\longrightarrow} \mathcal{V}_{-}
$$

of relative critical points (of $F$ ) in $\mathcal{B}_{-}$is a finite flat morphism of degree $N_{-}=\operatorname{dim} H_{\mathrm{CR}}^{*}\left(\mathfrak{X}_{-}\right)$ (see also the discussion in Section 6.3.1), where $\widetilde{p r}$ is the map in (4.3). Along the small quantum cohomology locus $\mathcal{V}_{-}^{\mathrm{sm}}=\mathcal{V}_{-} \cap \mathcal{M}^{\mathrm{sm}}$, this family contains all relative critical points, i.e., $\mathcal{B}_{-} \cap$ $\widetilde{\mathrm{pr}}^{-1}\left(\mathcal{V}_{-}^{\mathrm{sm}} \times\{0\}\right)=\widetilde{\mathrm{pr}}^{-1}\left(\mathcal{V}_{-}^{\mathrm{sm}} \times\{0\}\right)$ (since the Brieskorn module $\mathrm{Bri}^{\mathrm{sm}}(F)$ has the rank $N_{-}$by Proposition 6.15). We define

$$
\begin{aligned}
& \mathcal{V}_{-}^{\mathrm{ss}}:=\left\{q \in \mathcal{V}_{-}: \text {the fibre of } \mathrm{Cr}_{-} \rightarrow \mathcal{V}_{-} \text {at } q \in \mathcal{V}_{-} \text {is reduced }\right\}, \\
& \mathcal{V}_{-}^{\mathrm{sm}, \mathrm{ss}}:=\mathcal{V}_{-}^{\mathrm{ss}} \cap \mathcal{M}^{\mathrm{sm}}, \\
& \mathcal{V}_{-, \mathbb{R}}^{\mathrm{sm}, \mathrm{ss}}:=\mathcal{V}_{-}^{\mathrm{sm}, \mathrm{ss}} \cap \mathcal{M}_{\mathbb{R}}^{\mathrm{sm}},
\end{aligned}
$$

where $\mathcal{V}_{-}^{\text {ss }}$ is open dense in $\mathcal{V}_{-}, \mathcal{V}_{-}^{\text {sm,ss }}$ is open dense in $\mathcal{V}_{-}^{\text {sm }}$ and $\mathcal{V}_{-, \mathbb{R}}^{\text {sm,ss }}$ is open dense in $\mathcal{V}_{-}^{\mathrm{sm}} \cap \mathcal{M}_{\mathbb{R}}^{\mathrm{sm}}$.

Choose $q_{0} \in \mathcal{V}_{-, \mathbb{R}}^{\mathrm{sm}, \mathrm{ss}}$. Let $\mathrm{cr}_{1}(q), \ldots, \mathrm{cr}_{N_{-}}(q)$ be all branches of critical points of $F_{q}$ contained in $\mathcal{B}_{-}$and defined in a neighbourhood of $q=q_{0}$ in $\mathcal{V}_{-}^{\text {ss }}$. We may assume that $\operatorname{cr}_{1}\left(q_{0}\right)=\operatorname{cr}_{\mathbb{R}}\left(q_{0}\right)$ and write $\mathbf{u}_{i}(q)=F_{q}\left(\mathrm{cr}_{i}(q)\right)$ as before. By combining the mirror isomorphism (7.4) and the formal decomposition of the analytified Brieskorn module in Proposition 6.10, we obtain a formal decomposition

$$
\widehat{\Phi}: \quad \operatorname{mir}_{-}^{*} \overline{\mathrm{QDM}^{\mathrm{an}}}\left(\mathfrak{X}_{-}\right) \cong \bigoplus_{i=1}^{N_{-}}\left(\mathcal{O}^{\mathrm{an}} \llbracket z \rrbracket, d+d\left(\mathbf{u}_{i} / z\right)\right)
$$

over a neighbourhood of $q_{0}$ in $\mathcal{V}_{-}^{\text {ss }}$. By Proposition 6.5 , for an admissible phase $\phi$ for $\left\{\mathbf{u}_{1}\left(q_{0}\right), \ldots\right.$, $\left.\mathbf{u}_{N_{-}}\left(q_{0}\right)\right\}$, we have a connected open neighbourhood $B$ of $q_{0}$ in $\mathcal{V}_{-}^{\text {ss }}$, a sector $I_{\phi}=\left\{\left(r, e^{\mathbf{i} \theta}\right)\right.$ : $\left.|\theta-\phi|<\frac{\pi}{2}+\epsilon\right\}$ (with small $\epsilon>0$ ) and an analytic lift of $\widehat{\Phi}$ :

$$
\widetilde{\Phi}_{\phi}^{-}:\left.\pi^{*} \operatorname{mir}_{-}^{*} \operatorname{QDM}^{\mathrm{an}}\left(\mathfrak{X}_{-}\right)\right|_{B \times I_{\phi}} \cong \bigoplus_{i=1}^{N_{-}}\left(\mathcal{A}_{B \times I_{\phi}}, d+d\left(\mathbf{u}_{i} / z\right)\right),
$$

where $\pi: \mathcal{V}_{-}^{\text {ss }} \times \widetilde{\mathbb{C}} \rightarrow \mathcal{V}_{-}^{\text {ss }} \times \mathbb{C}$ is the oriented real blow-up. By the uniqueness of the analytic lift, this coincides with the analytic lift of the Brieskorn module from Proposition 6.18 over the small quantum cohomology locus $B \cap \mathcal{M}^{\mathrm{sm}}$, via the mirror isomorphism (7.5). Each component of $\widehat{\Phi}, \widetilde{\Phi}_{\phi}^{-}$is ambiguous up to sign, but recall from Remark 7.9 that the sign of the first component (corresponding to the conifold point $\mathrm{cr}_{\mathbb{R}}(q)$ ) is determined canonically. We have the following

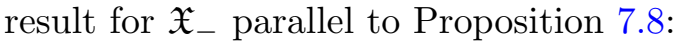


Proposition 7.13. For $q_{0} \in \mathcal{V}_{-, \mathbb{R}}^{\mathrm{sm}, \mathrm{ss}}$, there exists $\alpha_{0} \in(0, \pi / 2)$ such that the following holds. For every admissible phase $\phi \in\left(-\alpha_{0}, \alpha_{0}\right)$ for $\left\{\mathbf{u}_{1}\left(q_{0}\right), \ldots, \mathbf{u}_{N_{-}}\left(q_{0}\right)\right\}$, there exists a basis $\left\{V_{i}\right\}_{i=1}^{N_{-}}$ of $K\left(\mathfrak{X}_{-}\right)$with $V_{1}$ being the structure sheaf $\mathcal{O}$ of $\mathfrak{X}_{-}$, such that the corresponding flat section $s_{i}=\mathfrak{s}_{V_{i}}\left(\operatorname{mir}_{-}(q), z\right)$ satisfies $\widetilde{\Phi}_{\phi}^{-}\left(e^{\mathbf{u}_{i}(q) / z} s_{i}\right)=e_{i}$.

Proof. The same argument as Proposition 7.13 (using Theorem 7.12 in place of Theorem 7.5) shows that there exist $V_{i} \in K\left(\mathfrak{X}_{-}\right), i=1, \ldots, N_{-}$with $V_{1}=\mathcal{O}$ such that $\widetilde{\Phi}_{\phi}^{-}\left(e^{\mathbf{u}_{i}(q) / z} s_{i}\right)=e_{i}$ over $B \cap \mathcal{M}^{\text {sm }}$, where $s_{i}=\mathfrak{s}_{V_{i}}\left(\operatorname{mir}_{-}(q), z\right)$. It follows from the flatness of $s_{i}$ and $e^{-\mathbf{u}_{i}(q) / z} e_{i}$ that $\widetilde{\Phi}_{\phi}^{-}\left(e^{\mathbf{u}_{i}(q) / z} s_{i}\right)=e_{i}$ holds over the whole $B$.

\subsection{Inclusion of the local systems of Lefschetz thimbles}

The Brieskorn module over the small quantum cohomology locus is underlain by a local system of Lefschetz thimbles. In this section, we observe an inclusion of the local system over $\mathcal{M}^{\text {sm }}$ in a neighbourhood of $0_{-}=0_{\Sigma_{-}}\left(\right.$mirror to $\left.\mathfrak{X}_{-}\right)$to the local system over $\mathcal{M}$ (mirror to $\mathfrak{X}_{+}$) under a slide in the 'positive real' direction. The inclusion shall be identified with the pull-back $\varphi^{*}: K\left(\mathfrak{X}_{-}\right) \rightarrow K\left(\mathfrak{X}_{+}\right)$in $K$-theory in Section 7.5.

\subsubsection{Convergent and divergent critical branches}

Let $\mathcal{V}_{ \pm}$be (sufficiently small) analytic open neighbourhoods of $0_{ \pm} \in \mathcal{M}$ as in the previous Section 7.3. Recall the $\mathbb{C}^{\times}$-action on pr: $\mathcal{Y} \rightarrow \mathcal{M}$ generated by the Euler vector field considered in Sections 5.3-5.4. As discussed there, we may assume that $\mathcal{V}_{ \pm}$is $\mathbb{C}^{\times}$-invariant, because the mirror map mir $_{ \pm}$, the mirror isomorphism $\mathrm{Mir}_{ \pm}$and the analytified Brieskorn module can be extended to the orbit $\mathbb{C}^{\times} \mathcal{V}_{ \pm}\left(\mathcal{V}_{ \pm}\right.$is the intersection of $\mathcal{U}_{ \pm} \subset \mathcal{M} \times$ Lie $\mathbb{T}$ in Section 5.4 with $\mathcal{M} \times\{0\}$ ). Let $\mathrm{Cr}_{ \pm} \rightarrow \mathcal{V}_{ \pm}$denote the (finite, flat) family of relative critical points over $\mathcal{V}_{ \pm}$:

$$
\begin{aligned}
& \mathrm{Cr}_{+}=\left\{p \in \operatorname{pr}^{-1}\left(\mathcal{V}_{+}\right): x_{i} \frac{\partial F}{\partial x_{i}}(p)=0(\forall i)\right\}=\widetilde{\mathrm{pr}}^{-1}\left(\mathcal{V}_{+} \times\{0\}\right), \\
& \mathrm{Cr}_{-}=\left\{p \in \mathcal{B}_{-} \cap \operatorname{pr}^{-1}\left(\mathcal{V}_{-}\right): x_{i} \frac{\partial F}{\partial x_{i}}(p)=0(\forall i)\right\}=\widetilde{\operatorname{pr}}^{-1}\left(\mathcal{V}_{-} \times\{0\}\right) \cap \mathcal{B}_{-},
\end{aligned}
$$

where $\mathcal{B}_{-}$is the subset of $\mathcal{Y}$ appearing in the construction of $\overline{\operatorname{Bri}^{\text {an }}}(F)_{\boldsymbol{\Sigma}_{-}}$in Section 4.3 and $\widetilde{\text { pr }}$ is the map in (4.3). Since all the relative critical points over $\mathcal{V}_{+}$are contained in $\mathcal{B}_{+}$, we do not need to take the intersection with $\mathcal{B}_{+}$in the first formula. By Lemma 5.11, we have an open subset $\mathcal{V}_{0}=\mathcal{U}_{0} \cap(\mathcal{M} \times\{0\}) \subset \mathcal{V}_{+} \cap \mathcal{V}_{-}$containing $\mathcal{C} \backslash\left\{0_{+}, 0_{-}\right\}$such that the ramified covering $\mathrm{Cr}_{+} \mid \mathcal{V}_{0} \rightarrow \mathcal{V}_{0}$ decomposes as:

$$
\mathrm{Cr}_{+}\left|\mathcal{V}_{0}=\mathrm{Cr}_{-}\right| \mathcal{V}_{0} \sqcup \mathcal{D},
$$

where $\mathcal{D} \subset \mathrm{Cr}_{+}$is an open subset giving a subcover of $\mathrm{Cr}_{+} \mid \mathcal{V}_{0}$. By taking the $\mathbb{C}^{\times}$-orbit, we may assume that $\mathcal{V}_{0}$ is also $\mathbb{C}^{\times}$-invariant. The subcover $\mathcal{D} \rightarrow \mathcal{V}_{0}$ consists of branches of critical points that diverge at $0_{-}$. We call critical points corresponding to $\mathcal{D}$ divergent and those corresponding to $\mathrm{Cr}_{-}$convergent. Among the critical points over $\mathcal{C}$ described in Proposition 7.2, those corresponding to $\gamma \neq 0$ are divergent.

\subsubsection{Local co-ordinate system around $0_{-}=0_{\Sigma_{-}}$}

Recall from Proposition 3.15 that the local chart of $\mathcal{M}$ around $0_{-}$is given by

$$
\mathcal{M}_{-}=\left[\widetilde{\mathcal{M}}_{-} / \operatorname{Pic}^{\text {st }}\left(\mathfrak{X}_{-}\right)\right] \quad \text { with } \quad \widetilde{\mathcal{M}}_{-}=\operatorname{Spec}\left(\mathbb{C}\left[\boldsymbol{\Lambda}\left(\boldsymbol{\Sigma}_{-}\right)_{+}\right]\right)
$$


Set $R_{-}=R\left(\boldsymbol{\Sigma}_{-}\right)$. By the decomposition (3.16), we have $\boldsymbol{\Lambda}\left(\boldsymbol{\Sigma}_{-}\right)_{+} \cong \boldsymbol{\Lambda}_{+}^{\boldsymbol{\Sigma}_{-}} \times\left(\mathbb{Z}_{\geq 0}\right)^{S \backslash R_{-}}$. Let $\boldsymbol{\Lambda}^{\prime}\left(\boldsymbol{\Sigma}_{-}\right)_{+} \cong \boldsymbol{\Lambda}_{+}^{\boldsymbol{\Sigma}_{-}} \times\left(\mathbb{Z}_{\geq 0}\right)^{S_{-} \backslash R_{-}}$denote the monoid that we obtain from $\boldsymbol{\Lambda}\left(\boldsymbol{\Sigma}_{-}\right)_{+}$by replacing $S$ with $S_{-}$. Then we can decompose $\widetilde{\mathcal{M}}_{-}$as

$$
\widetilde{\mathcal{M}}_{-}=\operatorname{Spec}\left(\mathbb{C}\left[\boldsymbol{\Lambda}^{\prime}\left(\boldsymbol{\Sigma}_{-}\right)_{+}\right]\right) \times \mathbb{C}^{\{\hat{b}\}} .
$$

We denote by $(q, t)=\left(q, t_{\hat{b}}\right)$ a point on $\widetilde{\mathcal{M}}_{-}$, where $q \in \operatorname{Spec}\left(\mathbb{C}\left[\boldsymbol{\Lambda}^{\prime}\left(\boldsymbol{\Sigma}_{-}\right)_{+}\right]\right)$. Note that the local chart of $\mathcal{M}^{\mathrm{sm}}$ around $0_{-}$is the substack $\{t=0\}$ of $\mathcal{M}_{-}$:

$$
\mathcal{M}_{-}^{\mathrm{sm}}=\left[\widetilde{\mathcal{M}}_{-}^{\mathrm{sm}} / \operatorname{Pic}^{\mathrm{st}}\left(\mathfrak{X}_{-}\right)\right] \quad \text { with } \quad \widetilde{\mathcal{M}}_{-}^{\mathrm{sm}}=\operatorname{Spec}\left(\mathbb{C}\left[\boldsymbol{\Lambda}^{\prime}\left(\boldsymbol{\Sigma}_{-}\right)_{+}\right]\right) .
$$

Recall that $\operatorname{Pic}^{\text {st }}\left(\mathfrak{X}_{-}\right)$acts on the chart $\widetilde{\mathcal{M}}_{-}$(7.6) by the age pairing $(3.18)$; since $t_{\hat{b}}=q^{-\mathbf{w}}$ and $\mathbf{w} \in \mathbb{L}, \mathrm{Pic}^{\text {st }}\left(\mathfrak{X}_{-}\right)$acts trivially on the last co-ordinate $t_{\hat{b}}$.

On the chart (7.6), the $\mathbb{C}^{\times}$-action generated by the Euler vector field is given by

$$
s \cdot(q, t)=\left(s \cdot q, s^{-J} t\right),
$$

where $J=\left(\sum_{b \in M_{+}} D_{b} \cdot \mathbf{w}\right)-1$ (as in Proposition 7.2). Note that the $\mathbb{C}^{\times}$-weight of the coordinate $q^{\lambda}$ with $\lambda \in \boldsymbol{\Lambda}^{\prime}\left(\boldsymbol{\Sigma}_{-}\right)_{+}$is non-negative by Lemma A.5.

\subsubsection{Sliding out $\mathcal{M}^{\text {sm }}$}

Let $\mathcal{C} \subset \mathcal{M}$ be the toric curve connecting $0_{-}$and $0_{+}$as in Section 7.2 ; in the above chart $\widetilde{\mathcal{M}}_{-}$, $\mathcal{C}$ consists of points $(0, t)$. By Proposition 7.2 , we can choose a closed polydisc $\widetilde{\mathbb{D}} \subset\left\{t_{\hat{b}}=1\right\} \subset$ $\widetilde{\mathcal{M}}_{-}$of radius $0<\varepsilon<1$ centred at $(0,1) \in \mathcal{C}$ such that

- $\widetilde{\mathbb{D}}=\widetilde{\mathbb{D}}_{\mathrm{sm}} \times\{1\}$ with $\widetilde{\mathbb{D}}_{\mathrm{sm}} \subset \widetilde{\mathcal{M}}_{-}^{\text {sm }}=\operatorname{Spec}\left(\mathbb{C}\left[\boldsymbol{\Lambda}^{\prime}\left(\boldsymbol{\Sigma}_{-}\right)_{+}\right]\right)$a neighbourhood of $0_{-}$given by

$$
\widetilde{\mathbb{D}}_{\mathrm{sm}}:=\left\{q:\left|q^{\lambda}\right| \leq \varepsilon, \forall \lambda \in \boldsymbol{\Lambda}^{\prime}\left(\boldsymbol{\Sigma}_{-}\right)_{+} \backslash\{0\}\right\} ;
$$

- $\mathbb{D}:=\widetilde{\mathbb{D}} / \mathrm{Pic}^{\mathrm{st}}\left(\mathfrak{X}_{-}\right)$is contained in $\mathcal{V}_{0}$;

- $\mathbb{D}_{\mathrm{sm}}:=\widetilde{\mathbb{D}}_{\mathrm{sm}} / \mathrm{Pic}^{\mathrm{st}}\left(\mathfrak{X}_{-}\right)$is contained in $\mathcal{V}_{-}$(when regarded as a subset of $\left.\mathcal{M}_{-}^{\mathrm{sm}}\right)$;

- $F\left(\mathrm{Cr}_{-} \cap \mathrm{pr}^{-1}(\mathbb{D})\right) \subset B_{\rho_{0}}(0)$;

- $F\left(\mathcal{D} \cap \operatorname{pr}^{-1}(\mathbb{D})\right) \subset \bigcup_{k=1}^{J} B_{\rho_{1}}\left(3 \rho_{0} e^{(2 k-1) \pi \mathbf{i} / J}\right)$,

where $\rho_{0}:=\frac{1}{3} K^{-1 / J}, \rho_{1}:=\min \left(1,3 \sin \left(\frac{\pi}{2 J}\right)\right) \rho_{0}, B_{\rho}(z)$ denotes the open disc of radius $\rho$ centred at $z \in \mathbb{C}$, and $K, J$ are as in Proposition 7.2. The last two conditions imply that convergent critical values over $\mathbb{D}$ are contained in $\left\{|\mathbf{u}|<\rho_{0}\right\}$ and divergent critical values over $\mathbb{D}$ are contained in $\left\{|\mathbf{u}|>2 \rho_{0}\right\}$ and away from the sector $-\frac{\pi}{2 J} \leq \arg (\mathbf{u}) \leq \frac{\pi}{2 J}$ with vertex at the origin, see Fig. 10. See also Fig. 11.

Definition 7.14. Define the sliding map $\mathrm{s}_{t}: \mathbb{D}_{\mathrm{sm}} \rightarrow \mathcal{M}_{-}$with $0 \leq t \leq 1$ by $\mathrm{s}_{t}(q)=(q, t)$. Note that the map $\tilde{\mathbf{s}}_{t}: \widetilde{\mathbb{D}}_{\mathrm{sm}} \rightarrow \widetilde{\mathcal{M}}_{-}, q \mapsto(q, t)$ between the uniformizing charts is $\operatorname{Pic}^{\mathrm{st}}\left(\mathfrak{X}_{-}\right)$equivariant and thus descends to the map $s_{t}$. Note also that $\mathbf{s}_{0}=\operatorname{id}_{\mathbb{D}_{\mathrm{sm}}}$.

\section{Lemma 7.15.}

(1) For $0<t \leq 1$ and $q \in \mathbb{D}_{\mathrm{sm}}^{\times}:=\mathbb{D}_{\mathrm{sm}} \cap \mathcal{M}^{\mathrm{sm}, \times}$, we have $\mathrm{s}_{t}(q) \in \mathcal{M}^{\times}$.

(2) For $0<t \leq 1$, we have $\operatorname{Im}\left(\mathbf{s}_{t}\right) \subset t^{-1 / J} \cdot \mathbb{D} \subset \mathcal{V}_{0}$, where $t^{-1 / J} \cdot(-)$ denotes the $\mathbb{C}^{\times}$-action generated by the Euler vector field. 


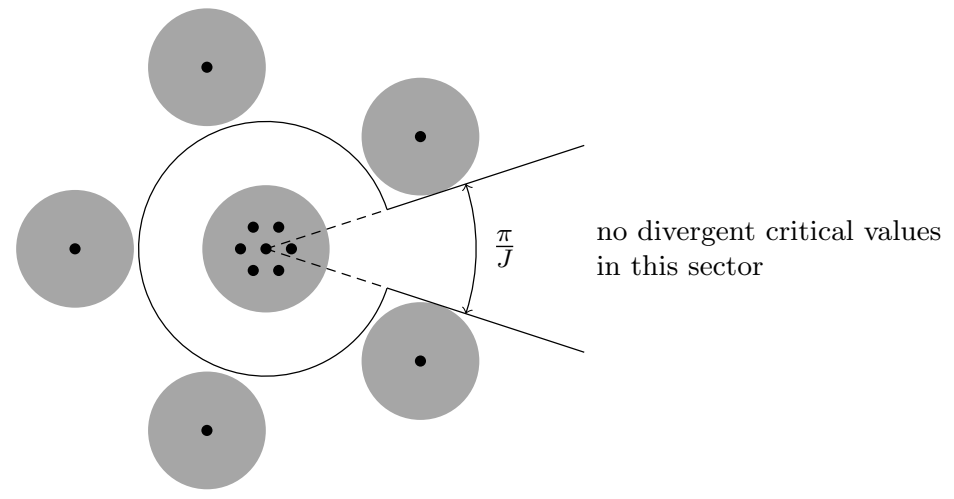

Figure 10. Distribution of critical values over $\mathbb{D}$ for $J=5$ : convergent critical values are in the shaded disc of radius $\rho_{0}$ in the centre; divergent critical values are in the 'satellite' discs.

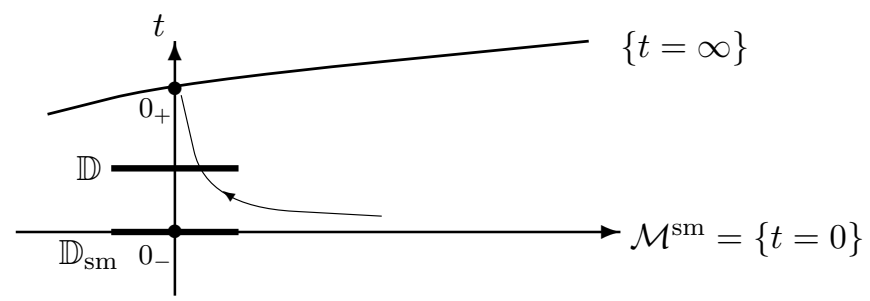

Figure 11. The base space $\mathcal{M}$ of the LG model and the $\operatorname{discs} \mathbb{D}, \mathbb{D}_{\mathrm{sm}}$ : the vertical $t$-axis is the curve $\mathcal{C}$ connecting $0_{+}$and $0_{-}$; the thin curve denotes the negative Euler flow (the flow as $s \rightarrow 0$ in (7.7)).

(3) There exists a constant $\rho_{2}>0$ such that convergent critical values over $\operatorname{Im}\left(\mathbf{s}_{t}\right)$ are contained in $B_{\rho_{2}}(0) \cap t^{-1 / J} \cdot B_{\rho_{0}}(0)$ and divergent critical values over $\operatorname{Im}\left(\mathbf{s}_{t}\right)$ are contained in $\bigcup_{k=1}^{J} t^{-1 / J} \cdot B_{\rho_{1}}\left(3 \rho_{0} e^{(2 k-1) \pi \mathbf{i} / J}\right)$.

Proof. Part (1) is obvious. By $(7.7), t^{1 / J} \cdot \operatorname{Im}\left(\mathbf{s}_{t}\right)$ consists of points $\left(t^{1 / J} \cdot q, 1\right)$ with $q \in \widetilde{\mathbb{D}}_{\mathrm{sm}}$. Since $0<t \leq 1$ and the $\mathbb{C}^{\times}$-weights on $\mathbb{C}\left[\boldsymbol{\Lambda}^{\prime}\left(\boldsymbol{\Sigma}_{-}\right)_{+}\right]$are non-negative, $\left|\left(t^{1 / J} \cdot q\right)^{\lambda}\right| \leq\left|q^{\lambda}\right| \leq \varepsilon$ for $q \in \widetilde{\mathbb{D}}_{\mathrm{sm}}$ and $\lambda \in \boldsymbol{\Lambda}^{\prime}\left(\boldsymbol{\Sigma}_{-}\right)_{+}$. Hence $t^{1 / J} \cdot \operatorname{Im}\left(\mathbf{s}_{t}\right) \subset \mathbb{D}$. Part (2) follows. Part (2) and the fact that the action of $t^{-1 / J}$ scales the critical values by $t^{-1 / J}$ imply that convergent critical values over $\operatorname{Im}\left(\mathbf{s}_{t}\right)$ are contained in $t^{-1 / J} \cdot B_{\rho_{0}}(0)$ and divergent ones over $\operatorname{Im}\left(\mathbf{s}_{t}\right)$ are contained in $\bigcup_{k=1}^{J} t^{-1 / J} \cdot B_{\rho_{1}}\left(3 \rho_{0} e^{(2 k-1) \pi \mathbf{i} / J}\right)$. Because the convergent critical branches form a proper family $\mathrm{Cr}_{-} \rightarrow \mathcal{V}_{-}$, the corresponding critical values are uniformly bounded in a neighbourhood of $\mathbb{D}_{\mathrm{sm}}=\operatorname{Im}\left(\mathbf{s}_{0}\right)$. Part (3) follows.

\subsubsection{Kouchnirenko's condition and a local system of Lefschetz thimbles}

Let $(q, t)=\left(q, t_{\hat{b}}\right)$ be the local co-ordinates on $\mathcal{M}_{-}$in Section 7.4.2. By abuse of notation, we mean by $q$ (resp. $(q, t))$ either a point on the uniformizing chart $\widetilde{\mathcal{M}}_{-}^{\text {sm }}$ (resp. $\widetilde{\mathcal{M}}_{-}$) or its image in $\mathcal{M}_{-}^{\text {sm }}$ (resp. $\mathcal{M}_{-}$) depending on the context. We write $\mathcal{Y}_{q, t}:=\operatorname{pr}^{-1}(q, t) \subset \mathcal{Y}, \mathcal{Y}_{q}^{\mathrm{sm}}:=$ $\operatorname{pr}^{-1}(q) \subset \mathcal{Y}^{\mathrm{sm}}$ for the fibres of the LG model; note that $\mathcal{Y}_{q}^{\mathrm{sm}}=\mathcal{Y}_{q, 0}$. The LG potential $F_{q, t}=\left.F\right|_{\mathcal{Y}_{q, t}}$ with $q \in \mathcal{M}^{\mathrm{sm}, \times}$ is a Laurent polynomial of the form (cf. (3.22))

$$
F_{q, t}=\sum_{b \in S} u_{b}=\left(\sum_{b \in S_{-}} q^{\ell_{b}} x^{b}\right)+t q^{\hat{\ell}} x^{\hat{b}} .
$$


Following Kouchnirenko [75, Définition 1.19], we make the following definition:

Definition 7.16 ([75]). Let $T \subset \mathbf{N}$ be a finite set and let $f(x)=\sum_{b \in T} c_{b} x^{b}$ be a Laurent polynomial function on $\operatorname{Hom}\left(\mathbf{N}, \mathbb{C}^{\times}\right)$. Suppose that $c_{b} \neq 0$ for all $b \in T$ and that the Newton polytope $\Delta$ of $f$, i.e., the convex hull of $\{\bar{b}: b \in T\}$ in $\mathbf{N}_{\mathbb{R}}$, contains the origin in its interior. We say that $f$ is (Newton) non-degenerate if for every proper face $\diamond$ of $\Delta$ (of any dimension), $f_{\diamond}(x):=\sum_{b \in T \cap \diamond} c_{b} x^{b}$ has no critical points on $\operatorname{Hom}\left(\mathbf{N}, \mathbb{C}^{\times}\right)$.

We are interested in the case where $T$ is $S$ or $S_{-}$, and $f$ is $F_{q, t}$ with $(q, t) \in \mathcal{M}^{\times}$or $F_{q, 0}$ with $q \in \mathcal{M}^{\mathrm{sm}, \times}$. The compactness of $\mathfrak{X}_{ \pm}$implies that the Newton polytope contains the origin in its interior in these cases. We define

$$
\begin{aligned}
& \mathcal{M}^{\text {sm,nd }}:=\left\{q \in \mathcal{M}^{\mathrm{sm}, \times}: F_{q, 0} \text { is Newton non-degenerate }\right\} \\
& \mathcal{M}^{\text {nd }}:=\left\{(q, t) \in \mathcal{M}^{\times}: F_{q, t} \text { is Newton non-degenerate }\right\} .
\end{aligned}
$$

\section{Proposition 7.17.}

(1) $\mathcal{M}^{\text {sm,nd }}$ is Zariski-open and dense in $\mathcal{M}^{\mathrm{sm}, \times}$.

(2) There exists a neighbourhood $B$ of $0_{-}$in $\mathcal{M}^{\mathrm{sm}}$ such that $\mathcal{M}^{\mathrm{sm}, \mathrm{nd}}$ contains $B \cap \mathcal{M}^{\mathrm{sm}, \times}$.

(3) The same conclusions as (1), (2) hold for $\mathcal{M}^{\text {nd }}$ when we replace $\mathcal{M}^{\mathrm{sm}, \times}$ with $\mathcal{M}^{\times}$and $0_{-}$ with $0_{+}$.

Proof. Part (1) is due to Kouchnirenko [75, Théorème 6.1]. Part (2) follows from the proof of [62, Lemma 3.8]; there we stated and proved a similar result using a particular co-ordinate system $\mathbb{C}^{r} \rightarrow \mathcal{M}_{-}^{\text {sm }}$, but the argument works verbatim for the possibly singular base $\mathcal{M}_{-}^{\text {sm }}$.

Remark 7.18. In view of Proposition 7.17(2), after shrinking the open sets $\mathcal{V}_{ \pm}$in Section 7.4.1 if necessary, we may assume that $\mathcal{V}_{+} \cap \mathcal{M}^{\times} \subset \mathcal{M}^{\text {nd }}$ and $\mathcal{V}_{-} \cap \mathcal{M}^{\text {sm, } \times} \subset \mathcal{M}^{\text {sm,nd }}$. This is possible because $\mathcal{V}_{ \pm}$was given as the $\mathbb{C}^{\times}$-orbit of an arbitrarily small neighbourhood of $0_{ \pm}$, and the non-degenerate loci $\mathcal{M}^{\text {sm,nd }}, \mathcal{M}^{\text {nd }}$ are $\mathbb{C}^{\times}$-invariant.

We define a function $H(x)$ of $\left(x_{1}, \ldots, x_{n}\right) \in\left(\mathbb{C}^{\times}\right)^{n}$ as

$$
H(x):=\sqrt{\sum_{b \in S_{-}}\left|x^{b}\right|^{2}} .
$$

Since the convex hull $\Delta_{-}$of $\left\{\bar{b}: b \in S_{-}\right\}$contains the origin in its interior, $H(x)$ is proper and bounded from below. By the splitting $\varsigma$ chosen at the end of Section 7.1, we can regard $x_{1}, \ldots, x_{n}$ and $H(x)$ as functions on the preimage of $\left\{(q, t): q \in \mathcal{M}^{\text {sm, }} \times\right\} \subset \mathcal{M}_{-}$under pr: $\mathcal{Y} \rightarrow \mathcal{M}$.

\section{Proposition 7.19.}

(1) For every compact set $K \subset \mathcal{M}^{\text {sm,nd }}$, there exist a compact set $B \subset \operatorname{pr}^{-1}(K)$ and $\epsilon>0$ such that

$$
\left\|d F_{q, 0}(x)\right\| \geq \epsilon H(x) \quad \text { for all } q \in K \text { and } x \in \mathcal{Y}_{q}^{\mathrm{sm}} \backslash B
$$

where $\|\cdot\|$ is the norm with respect to the Kähler metric $\frac{\mathbf{i}}{2} \sum_{j=1}^{n} d \log x_{j} \wedge d \overline{\log x_{j}}$, i.e., $\left\|d F_{q, 0}(x)\right\|=\left(\sum_{j=1}^{n}\left|\partial F_{q, 0} / \partial \log x_{j}\right|^{2}\right)^{1 / 2}$. 
(2) The same estimate holds for $\left\|d F_{q, t}\right\|$ by replacing $\mathcal{M}^{\mathrm{sm}, \mathrm{nd}}$ with $\mathcal{M}^{\mathrm{nd}}, \mathcal{Y}^{\mathrm{sm}}$ with $\mathcal{Y}$, and $H(x)$ with $\left(\sum_{b \in S}\left|x^{b}\right|^{2}\right)^{1 / 2}$.

Proof. This is a refinement of [62, Lemma 3.11], which says that $\left\|d F_{q, 0}(x)\right\|$ is proper on $\mathrm{pr}^{-1}(K)$. In fact, a slight modification of the argument there yields a proof of the proposition.

It suffices to show that there exists $\epsilon>0$ such that $\left\{x \in \operatorname{pr}^{-1}(K):\left\|d F_{q, 0}(x)\right\| \leq \epsilon H(x)\right\}$ is compact. Suppose on the contrary that $\left\{x \in \operatorname{pr}^{-1}(K):\left\|d F_{q, 0}(x)\right\| \leq H(x) / k\right\}$ is non-compact for all $k \geq 1$. Then we can find $q(k) \in K$ and $x(k) \in \mathcal{Y}_{q(k)}^{\text {sm }}$ such that $\left\|d F_{q(k), 0}(x(k))\right\| \leq$ $H(x(k)) / k$ and $H(x(k)) \geq k$. Passing to a subsequence we may assume that $q(k)$ converges in $K$; we may also assume that we can label elements of $S_{-}$as $\{b(1), b(2), \ldots, b(m)\}$ so that $\left|x(k)^{b(1)}\right| \geq\left|x(k)^{b(2)}\right| \geq \cdots \geq\left|x(k)^{b(m)}\right|$ holds for all $k$. Since $H(x(k)) \leq \sqrt{m}\left|x(k)^{b(1)}\right|$, we have $\lim _{k \rightarrow \infty}\left|x(k)^{b(1)}\right|=\infty$ and

$$
\lim _{k \rightarrow \infty} \frac{\left\|d F_{q(k), 0}(x(k))\right\|}{\left|x(k)^{b(1)}\right|}=0 .
$$

Now the argument after the second displayed equation in [62, Section A.2] yields a contradiction. The proposition follows.

Remark 7.20. Proposition 7.19 shows that the relative critical set of $F$ is proper over the non-degenerate loci $\mathcal{M}^{\text {sm,nd }}, \mathcal{M}^{\text {nd }}$.

\section{Corollary 7.21.}

(1) The family $\left\{F_{q, 0}^{-1}(\mathbf{u}) \subset \mathcal{Y}_{q}^{\mathrm{sm}}\right\}_{q, \mathbf{u}}$ of affine varieties is a locally trivial family of $C^{\infty}$ manifolds over $\left\{(q, \mathbf{u}): q \in \mathcal{M}^{\mathrm{sm}, \mathrm{nd}}, \mathbf{u}\right.$ is a regular value of $\left.F_{q, 0}\right\}$.

(2) The same conclusion holds for $F_{q, t}^{-1}(\mathbf{u}) \subset \mathcal{Y}_{q, t}$ when we replace $\mathcal{M}^{\mathrm{sm}, \mathrm{nd}}$ with $\mathcal{M}^{\mathrm{nd}}$.

Proof. Take $\left(q_{0}, \mathbf{u}_{0}\right)$ such that $q_{0} \in \mathcal{M}^{\text {sm,nd }}$ and $\mathbf{u}_{0}$ is a regular value of $F_{q_{0}, 0}$. Choose a sufficiently small co-ordinate neighbourhood $\left(B ; q^{1}, \ldots, q^{r}, \mathbf{u}\right)$ of $\left(q_{0}, \mathbf{u}_{0}\right)$ which does not intersect the discriminant locus. The ambient family $\bigcup_{(q, \mathbf{u}) \in B} \mathcal{Y}_{q}^{\mathrm{sm}}$ is trivial over $B$, and is identified with $B \times \operatorname{Hom}\left(\mathbf{N}, \mathbb{C}^{\times}\right)$through the co-ordinates $x_{1}, \ldots, x_{n}$. It suffices to shows that the co-ordinate vector fields ${ }^{20} \alpha \partial / \partial q^{i}, \alpha \partial / \partial \mathbf{u}$ with $\alpha \in \mathbb{C}$ on $B$ can be lifted to integrable vector fields tangent to the family $\bigcup_{(q, u) \in B} F_{q, 0}^{-1}(\mathbf{u})$. Lifts of $\alpha \partial / \partial q^{i}, \alpha \partial / \partial \mathbf{u}$ are given under the trivialization $\bigcup_{(q, \mathbf{u}) \in B} \mathcal{Y}_{q}^{\mathrm{sm}} \cong B \times \operatorname{Hom}\left(\mathbf{N}, \mathbb{C}^{\times}\right)$by

$$
\left(\alpha \frac{\partial}{\partial q^{i}},-\alpha \frac{\partial F_{q, 0}}{\partial q^{i}} \frac{\operatorname{grad} F_{q, 0}}{\left\|d F_{q, 0}\right\|^{2}}\right), \quad\left(\alpha \frac{\partial}{\partial \mathbf{u}}, \alpha \frac{\operatorname{grad} F_{q, 0}}{\left\|d F_{q, 0}\right\|^{2}}\right)
$$

where

$$
\operatorname{grad} F_{q, 0}:=\sum_{j=1}^{n} \overline{\frac{\partial F_{q, 0}}{\partial \log x_{j}}} \frac{\partial}{\partial \log x_{j}} .
$$

Since the potential $F_{q, 0}$ is of the form (7.8), we have $\left|\partial F_{q, 0} / \partial q^{i}\right| \leq C \cdot H(x)$ for some constant $C>0$ over $B$. Thus the estimate in Proposition 7.19 shows that these lifts are bounded on the family $\bigcup_{(q, \mathbf{u}) \in B} F_{q, 0}^{-1}(\mathbf{u})$. Therefore the flows of these vector fields exist as long as the corresponding flows on the base $B$ exists.

\footnotetext{
${ }^{20}$ Here we identify (real) vector fields of a complex manifold with $(1,0)$ vector fields.
} 
Proposition 7.19 implies that the improper function $\Re\left(F_{q, 0}(x)\right)$ satisfies the so-called PalaisSmale condition ${ }^{21}$ when $q \in \mathcal{M}^{\text {sm,nd }}$, and hence the usual Morse theory can be applied to it. It follows (see [62, Section 3.3.1]) that the relative homology group $H_{n}\left(\mathcal{Y}_{q}^{\text {sm }},\left\{x: \Re\left(F_{q, 0}(x)\right) \geq\right.\right.$ $M\} ; \mathbb{Z})$ is a free $\mathbb{Z}$-module of rank $\operatorname{dim} H_{\mathrm{CR}}^{*}\left(\mathfrak{X}_{-}\right)$when $M$ is large enough so that all critical values of $F_{q, 0}$ are contained in $\{\Re(z)<M\}$. This group is independent of the choice of sufficiently large $M$, and we denote it by $H_{n}\left(\mathcal{Y}_{q}^{\mathrm{sm}},\left\{x: \Re\left(F_{q, 0}(x)\right) \gg 0\right\} ; \mathbb{Z}\right)$. By the local triviality in Corollary 7.21 , we have

$$
\operatorname{Lef}_{q}^{\mathrm{sm}}:=H_{n}\left(\mathcal{Y}_{q}^{\mathrm{sm}},\left\{x: \Re\left(F_{q, 0}(x)\right) \gg 0\right\} ; \mathbb{Z}\right) \cong H_{n}\left(\mathcal{Y}_{q}^{\mathrm{sm}}, F_{q, 0}^{-1}(\mathbf{u}) ; \mathbb{Z}\right)
$$

for any $\mathbf{u}>0$ such that all critical values of $F_{q, 0}$ are contained in $\{z: \Re(z)<\mathbf{u}\}$, and this forms a local system over $\mathcal{M}^{\mathrm{sm} \text {,nd }}$. Similarly, the relative homology groups

$$
\operatorname{Lef}_{q, t}:=H_{n}\left(\mathcal{Y}_{q, t},\left\{x: \Re\left(F_{q, t}(x)\right) \gg 0\right\} ; \mathbb{Z}\right) \cong H_{n}\left(\mathcal{Y}_{q, t}, F_{q, t}^{-1}(\mathbf{u}) ; \mathbb{Z}\right)
$$

form a local system of rank $\operatorname{dim} H_{\mathrm{CR}}^{*}\left(\mathfrak{X}_{+}\right)$over $\mathcal{M}^{\text {nd }}$, where $\mathbf{u}>0$ is such that all critical values of $F_{q, t}$ are contained in $\{z: \Re(z)<\mathbf{u}\}$. These two local systems have different ranks and we will relate them below.

\subsubsection{Inclusion of the local systems: statement and proof}

Let $\mathbf{s}_{t}$ be the sliding map in Definition 7.14 and set $\mathbb{D}_{\mathrm{sm}}^{\times}:=\mathbb{D}_{\mathrm{sm}} \cap \mathcal{M}^{\mathrm{sm}, \times}$. Let $\mathbf{s}_{>0}$ denote the map $(0,1] \times \mathbb{D}_{\mathrm{sm}}^{\times} \rightarrow \mathcal{M}^{\text {nd }}$ given by $\mathbf{s}_{>0}(t, q)=\mathbf{s}_{t}(q)$. Since $(0,1]$ is contractible, we have

$$
\mathrm{s}_{>0}^{-1} \text { Lef } \cong \mathrm{p}^{-1}\left(\left.\left(\mathrm{~s}_{t}^{-1} \text { Lef }\right)\right|_{\mathbb{D}_{\mathrm{sm}}^{\times}}\right) \quad \forall t \in(0,1]
$$

where $\mathrm{p}:(0,1] \times \mathbb{D}_{\mathrm{sm}}^{\times} \rightarrow \mathbb{D}_{\mathrm{sm}}^{\times}$is the projection to the second factor. Let $\mathrm{j}:(0,1] \times \mathbb{D}_{\mathrm{sm}}^{\times} \rightarrow$ $[0,1] \times \mathbb{D}_{\mathrm{sm}}^{\times}$and $i: \mathbb{D}_{\mathrm{sm}}^{\times} \cong\{0\} \times \mathbb{D}_{\mathrm{sm}}^{\times} \rightarrow[0,1] \times \mathbb{D}_{\mathrm{sm}}^{\times}$denote the inclusions. Then we have

$$
i^{-1} j_{*} s_{>0}^{-1} \text { Lef }\left.\cong i^{-1} j_{*} p^{-1}\left(\left.\left(s_{t}^{-1} \text { Lef }\right)\right|_{\mathbb{D}_{\mathrm{sm}}^{\times}}\right) \cong\left(s_{t}^{-1} \text { Lef }\right)\right|_{\mathbb{D}_{\mathrm{sm}}^{\times}} .
$$

Theorem 7.22. We have an inclusion of the local systems:

$$
\iota: \quad \text { Lef }\left.^{\mathrm{sm}}\right|_{\mathbb{D}_{\mathrm{sm}}^{\times}} \rightarrow i^{-1} j_{*} \mathbf{s}_{>0}^{-1} \text { Lef }\left.\cong\left(\mathbf{s}_{t}^{-1} \text { Lef }\right)\right|_{\mathbb{D}_{\mathrm{sm}}^{\times}}, \quad t \in(0,1] .
$$

The map ८ maps the positive real Lefschetz thimble (introduced in Section 7.3) to the positive real one, i.e., $\iota\left(\Gamma_{\mathbb{R}}(q)\right)=\Gamma_{\mathbb{R}}\left(\mathbf{s}_{t}(q)\right)$ when $q \in \mathbb{D}_{\mathrm{sm}}^{\times} \cap \mathcal{M}_{\mathbb{R}}^{\mathrm{sm}}$.

Remark 7.23. By Remark 7.18, we may assume that $\mathcal{V}_{+} \cap \mathcal{M}^{\times} \subset \mathcal{M}^{\text {nd }}$ and $\mathcal{V}_{-} \cap \mathcal{M}^{\text {sm, } \times} \subset$ $\mathcal{M}^{\text {sm,nd }}$. Then by the choice of $\mathbb{D}_{\mathrm{sm}}, \mathbb{D}$ in Section 7.4.3 and Lemma 7.15 , we have that $\mathbb{D}_{\mathrm{sm}}^{\times} \subset$ $\mathcal{M}^{\text {sm,nd }}$ and $\mathrm{s}_{t}\left(\mathbb{D}_{\mathrm{sm}}^{\times}\right) \subset \mathcal{V}_{0} \cap \mathcal{M}^{\times} \subset \mathcal{V}_{+} \cap \mathcal{M}^{\times} \subset \mathcal{M}^{\text {nd }}$ for $t>0$. Hence Lef ${ }^{\text {sm }}$ and Lef are local systems over $\mathbb{D}_{\mathrm{sm}}^{\times}$and $\operatorname{Im}\left(\mathbf{s}_{t}\right)$ (with $0<t \leq 1$ ) respectively, and the statement of Theorem 7.22 makes sense.

For $(q, t) \in \mathcal{M}_{-}$and $\eta>0$, we set

$$
A_{q, t}(\eta):=\mathcal{Y}_{q, t} \cap\{H(x) \leq \eta\},
$$

where $H(x)$ is as in (7.9). When $\eta>\min _{x \in \mathcal{Y}_{q, t}} H(x), A_{q, t}(\eta)$ is a compact region such that the inclusion $A_{q, t}(\eta) \hookrightarrow \mathcal{Y}_{q, t}$ is a homotopy equivalence. Indeed, via the $\operatorname{Hom}\left(\mathbf{N}, S^{1}\right)$-action, we have

$$
A_{q, t}(\eta) \cong \operatorname{Hom}\left(\mathbf{N}, S^{1}\right) \times\left\{\left(x_{1}, \ldots, x_{n}\right) \in\left(\mathbb{R}_{>0}\right)^{n}: H(x) \leq \eta\right\}
$$

\footnotetext{
${ }^{21}\left\|d F_{q, 0}(x)\right\|$ is bounded away from zero outside a neighbourhood of the critical set.
} 
and the second factor is contractible since $\left.H\right|_{\left(\mathbb{R}_{>0}\right)^{n}}$ is strictly convex. For any $(q, \mathbf{u}) \in \mathcal{M}^{\mathrm{sm}, \times} \times \mathbb{C}$, the real algebraic function $H(x)^{2}$ restricted to $F_{q, 0}^{-1}(\mathbf{u})$ has finitely many critical values by [84, Corollary 2.8], and thus there exists $\eta_{0}>0$ such that $F_{q, 0}^{-1}(\mathbf{u})$ and $\partial A_{q, 0}(\eta)$ intersect transversally for all $\eta$ with $\eta \geq \eta_{0}$. We will show in the following lemma that such an $\eta_{0}$ can be chosen independently of $(q, \mathbf{u})$ as far as $(q, \mathbf{u})$ varies in a compact subset of $\mathcal{M}^{\text {sm,nd }} \times \mathbb{C}$. We note that Nemethi-Zaharia [85] and Parusinski [89] have obtained analogous results for a single polynomial function on $\mathbb{C}^{n}$ (and the proof is similar).

\section{Lemma 7.24.}

(1) For a compact subset $K \subset \mathcal{M}^{\mathrm{sm}, \mathrm{nd}} \times \mathbb{C}$, there exists $\eta_{0}$ such that for all $\eta \geq \eta_{0}$ and all $(q, \mathbf{u}) \in K, F_{q, 0}^{-1}(\mathbf{u})$ and $\partial A_{q, 0}(\eta)$ intersect transversally.

(2) The same result on the transversality of $F_{q, t}^{-1}(\mathbf{u})$ and $\partial A_{q, t}(\eta)$ holds when we replace $\mathcal{M}^{\mathrm{sm}, \mathrm{nd}}$ with $\mathcal{M}^{\text {nd }}$.

The proof of Lemma 7.24 will be given in Appendix B. In the situation of Lemma 7.24(1), $A_{q, 0}(\eta) \cap F_{q, 0}^{-1}(\mathbf{u})$ is a deformation retract of $F_{q, 0}^{-1}(\mathbf{u})$ for $\eta \geq \eta_{0}$; we can see this using the Morse flow for the function $H(x)$ on $F_{q, 0}^{-1}(\mathbf{u})$. In particular, the inclusion of pairs

$$
\left(A_{q, 0}(\eta), F_{q, 0}^{-1}(\mathbf{u}) \cap A_{q, 0}(\eta)\right) \rightarrow\left(\mathcal{Y}_{q}^{\mathrm{sm}}, F_{q, 0}^{-1}(\mathbf{u})\right)
$$

induces an isomorphism of relative homology. This fact will be used in the following proof.

Proof of Theorem 7.22. We shall construct an inclusion of local systems:

$$
\iota: \quad \text { Lef }\left.^{\mathrm{sm}}\right|_{\mathbb{D}_{\mathrm{sm}}^{\times}} \rightarrow \mathrm{i}^{-1} \mathrm{j}_{*} \mathrm{~s}_{>0}^{-1} \text { Lef. }
$$

Note that the stalk of $i^{-1} j_{*} \mathbf{s}_{>0}^{-1}$ Lef at $q_{0} \in \mathbb{D}_{\mathrm{sm}}^{\times}$consists of a Gauss-Manin flat family of relative homology classes in $\operatorname{Lef}_{\mathrm{s}_{t}(q)}$ with $t>0$ sufficiently small and $q$ in a small contractible neighbourhood of $q_{0}$ in $\mathcal{M}^{\text {sm,nd }}$. The construction of $\iota$ will be done in the following 5 steps.

(1) Construction of $\iota$ on the stalk at $q_{0} \in \mathbb{D}_{\mathrm{sm}}^{\times}$. Let $\rho_{2}>0$ be the constant in Lemma 7.15(3) and let $\mathbf{u}_{0}>\rho_{2}$ be such that all critical values of $F_{q_{0}, 0}$ are contained in $\left\{\mathbf{u}: \Re(\mathbf{u})<\mathbf{u}_{0}\right\}$. By Lemma 7.24, there exists $\eta_{0}>0$ such that $F_{q, 0}^{-1}(\mathbf{u}) \pitchfork \partial A_{q, 0}(\eta)$ for all $\eta \geq \eta_{0}$ and $(q, \mathbf{u})$ in a neighbourhood of $\left(q_{0}, \mathbf{u}_{0}\right)$. Then by the remark preceding the proof, the inclusion induces an isomorphism of relative homology (we use $\mathbb{Z}$ coefficients unless otherwise mentioned):

$$
H_{n}\left(A_{q_{0}, 0}\left(\eta_{0}\right), F_{q_{0}, 0}^{-1}\left(\mathbf{u}_{0}\right) \cap A_{q_{0}, 0}\left(\eta_{0}\right)\right) \stackrel{\cong}{\rightrightarrows} H_{n}\left(\mathcal{Y}_{q_{0}}^{\mathrm{sm}}, F_{q_{0}, 0}^{-1}\left(\mathbf{u}_{0}\right)\right) \cong \operatorname{Lef}_{q_{0}}^{\mathrm{sm}}
$$

Since $\left\{\partial A_{q, t}\left(\eta_{0}\right)\right\}_{q, t}$ is a proper family, $F_{q, t}^{-1}\left(\mathbf{u}_{0}\right)$ and $\partial A_{q, t}\left(\eta_{0}\right)$ intersect transversally for $(q, t)$ in a sufficiently small contractible neighbourhood $B$ of $\left(q_{0}, 0\right)$ in $\mathcal{M}_{-}$. The Ehresmann fibration theorem implies that $F_{q, t}^{-1}\left(\mathbf{u}_{0}\right) \cap A_{q, t}\left(\eta_{0}\right)$ is a trivial family of $C^{\infty}$-manifolds (with boundary) when $(q, t)$ varies in $B$. Therefore, whenever $\mathbf{s}_{t}(q)$ lies in $B$, the inclusion of pairs

$$
\left(A_{\mathbf{s}_{t}(q)}\left(\eta_{0}\right), F_{\mathbf{s}_{t}(q)}^{-1}\left(\mathbf{u}_{0}\right) \cap A_{\mathbf{s}_{t}(q)}\left(\eta_{0}\right)\right) \hookrightarrow\left(A_{B}\left(\eta_{0}\right), F^{-1}\left(\mathbf{u}_{0}\right) \cap A_{B}\left(\eta_{0}\right)\right)
$$

induces an isomorphism of relative homology, where we set $A_{B}\left(\eta_{0}\right):=\bigcup_{(q, t) \in B} A_{q, t}\left(\eta_{0}\right)$. Thus we obtain a Gauss-Manin flat isomorphism

$$
\begin{aligned}
& H_{n}\left(A_{B}\left(\eta_{0}\right), F^{-1}\left(\mathbf{u}_{0}\right) \cap A_{B}\left(\eta_{0}\right)\right) \\
& H_{n}\left(A_{q_{0}, 0}\left(\eta_{0}\right), F_{q_{0}, 0}^{-1}\left(\mathbf{u}_{0}\right) \cap A_{q_{0}, 0}\left(\eta_{0}\right)\right) \quad H_{n}\left(A_{\mathbf{s}_{t}(q)}\left(\eta_{0}\right), F_{\mathbf{s}_{t}(q)}^{-1}\left(\mathbf{u}_{0}\right) \cap A_{\mathbf{s}_{t}(q)}\left(\eta_{0}\right)\right)
\end{aligned}
$$

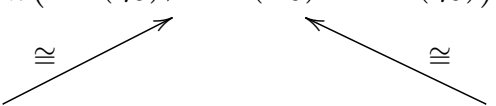


for $t>0$ sufficiently small and $q$ in a small neighbourhood of $q_{0}$. Composing (7.11), (7.12) and the natural map induced by the inclusion:

$$
H_{n}\left(A_{\mathbf{s}_{t}(q)}\left(\eta_{0}\right), F_{\mathbf{s}_{t}(q)}^{-1}\left(\mathbf{u}_{0}\right) \cap A_{\mathbf{s}_{t}(q)}\left(\eta_{0}\right)\right) \longrightarrow H_{n}\left(\mathcal{Y}_{\mathbf{s}_{t}(q)}, F_{\mathbf{s}_{t}(q)}^{-1}\left(\mathbf{u}_{0}\right)\right)
$$

we obtain

$$
\operatorname{Lef}_{q_{0}}^{\mathrm{sm}} \longrightarrow H_{n}\left(\mathcal{Y}_{\mathrm{s}_{t}(q)}, F_{\mathrm{s}_{t}(q)}^{-1}\left(\mathbf{u}_{0}\right)\right)
$$

Now recall from Lemma 7.15(3) that there are no critical values of $F_{\mathbf{s}_{t}(q)}$ in the region $\{\mathbf{u} \in \mathbb{C}$ : $\left.|\mathbf{u}| \geq \rho_{2}, \arg (\mathbf{u}) \in\left[-\frac{\pi}{2 J}, \frac{\pi}{2 J}\right]\right\}$. Since $\mathbf{u}_{0}>\rho_{2}$, the parallel transportation along a straight path defines a canonical isomorphism

$$
H_{n}\left(\mathcal{Y}_{\mathbf{s}_{t}(q)}, F_{\mathbf{s}_{t}(q)}^{-1}\left(\mathbf{u}_{0}\right)\right) \cong H_{n}\left(\mathcal{Y}_{\mathbf{s}_{t}(q)}, F_{\mathbf{s}_{t}(q)}^{-1}\left(\mathbf{u}_{1}\right)\right)
$$

for all $\mathbf{u}_{1}>\mathbf{u}_{0}$. Thus (7.13) gives a map $\operatorname{Lef}_{q_{0}}^{\mathrm{sm}} \rightarrow \operatorname{Lef}_{\mathrm{s}_{t}(q)}$ for $t>0$ sufficiently small and $q$ in a small neighbourhood of $q_{0}$. This map is Gauss-Manin flat as $(t, q)$ varies and defines the map $\iota_{q_{0}}$ on the stalks.

(2) Independence of the choice of $\eta_{0}, \mathbf{u}_{0}$. We show that the map $\iota_{q_{0}}$ is independent of the choices made. That replacing $\eta_{0}$ with a bigger $\eta_{1}>\eta_{0}$ does not change the map $\iota_{q_{0}}$ follows from the commutative diagram:

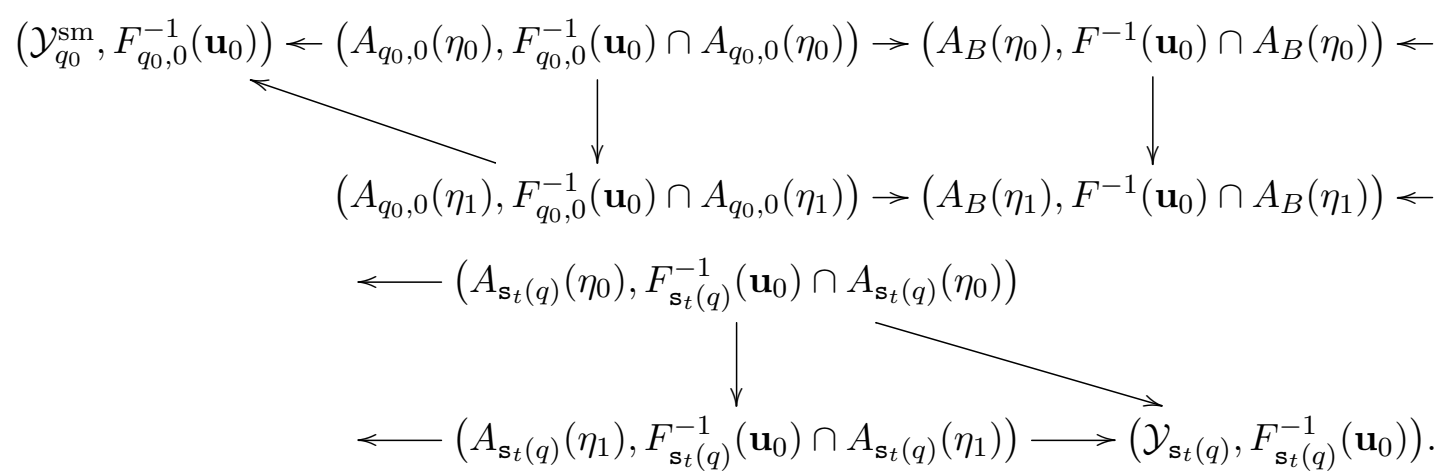

Next we show that the map is independent of $\mathbf{u}_{0}$. Suppose that we choose $\mathbf{u}_{1}>\mathbf{u}_{0}$ in place of $\mathbf{u}_{0}$. By Lemma 7.24 again, we can choose $\eta_{0}>0$ such that $F_{q, 0}^{-1}(\mathbf{u}) \pitchfork \partial A_{q, 0}(\eta)$ for all $\eta \geq \eta_{0}$, all $q$ in a neighbourhood of $q_{0}$ and all $\mathbf{u}$ in the interval $\left[\mathbf{u}_{0}, \mathbf{u}_{1}\right]$. Then the family of $C^{\infty}$-manifolds $F_{q, t}^{-1}(\mathbf{u}) \cap A_{q, t}\left(\eta_{0}\right)$ is trivial as $\mathbf{u}$ varies in $\left[\mathbf{u}_{0}, \mathbf{u}_{1}\right]$ and $(q, t)$ varies in a contractible neighbourhood $B$ of $\left(q_{0}, 0\right)$. The various maps in the construction of $\iota_{q_{0}}$ can be compared with the following sequence of maps:

$$
\begin{aligned}
\left(\mathcal{Y}_{q_{0}}^{\mathrm{sm}}\right. & \left.\times\left[\mathbf{u}_{0}, \mathbf{u}_{1}\right], F_{q_{0}, 0}^{-1}\left[\mathbf{u}_{0}, \mathbf{u}_{1}\right]\right) \\
& \leftarrow\left(A_{q_{0}, 0}\left(\eta_{0}\right) \times\left[\mathbf{u}_{0}, \mathbf{u}_{1}\right], F_{q_{0}, 0}^{-1}\left[\mathbf{u}_{0}, \mathbf{u}_{1}\right] \cap\left(A_{q_{0}, 0}\left(\eta_{0}\right) \times\left[\mathbf{u}_{0}, \mathbf{u}_{1}\right]\right)\right) \\
& \rightarrow\left(A_{B}\left(\eta_{0}\right) \times\left[\mathbf{u}_{0}, \mathbf{u}_{1}\right], F^{-1}\left[\mathbf{u}_{0}, \mathbf{u}_{1}\right] \cap\left(A_{B}\left(\eta_{0}\right) \times\left[\mathbf{u}_{0}, \mathbf{u}_{1}\right]\right)\right) \\
& \leftarrow\left(A_{\mathrm{s}_{t}(q)}\left(\eta_{0}\right) \times\left[\mathbf{u}_{0}, \mathbf{u}_{1}\right], F_{\mathrm{s}_{t}(q)}^{-1}\left[\mathbf{u}_{0}, \mathbf{u}_{1}\right] \cap\left(A_{\mathrm{s}_{t}(q)}\left(\eta_{0}\right) \times\left[\mathbf{u}_{0}, \mathbf{u}_{1}\right]\right)\right) \\
& \rightarrow\left(\mathcal{Y}_{\mathrm{s}_{t}(q)} \times\left[\mathbf{u}_{0}, \mathbf{u}_{1}\right], F_{\mathrm{s}_{t}(q)}^{-1}\left[\mathbf{u}_{0}, \mathbf{u}_{1}\right]\right)
\end{aligned}
$$

where $F_{q, t}^{-1}\left[\mathbf{u}_{0}, \mathbf{u}_{1}\right]$ is regarded as a subset of $\mathcal{Y}_{q, t} \times\left[\mathbf{u}_{0}, \mathbf{u}_{1}\right]$ via $x \mapsto\left(x, F_{q, t}(x)\right)$ and the maps between the first four pairs induce isomorphisms in relative homology. It follows that the different choices $\mathbf{u}_{0}, \mathbf{u}_{1}$ give the same map $\iota_{q_{0}}$. 
(3) Gauss-Manin flatness of $\iota$. This is obvious from the construction in (1); note that $q_{0}$ there can vary in a small open subset of $\mathbb{D}_{\mathrm{sm}}^{\times}$.

(4) Injectivity of $\iota$. Choose $q_{0} \in \mathbb{D}_{\mathrm{sm}}^{\times} \cap \mathcal{V}_{-}^{\mathrm{sm} \text {,ss }}$, where $\mathcal{V}_{-}^{\mathrm{sm}, \mathrm{ss}}$ is an open dense subset of $\mathcal{V}_{-}^{\mathrm{sm}}$ appearing in Section 7.3.2, so that all critical points $\mathrm{cr}_{1}, \ldots, \mathrm{cr}_{N_{-}}$of $F_{q_{0}, 0}$ are non-degenerate. It suffices to show that $\iota_{q_{0}}$ is injective. Let $\mathbf{u}_{0}>0$ be as in (1). In this case, a basis of Lef $q_{0}^{\text {sm }}$ is given by Lefschetz thimbles: setting $\mathbf{u}_{i}=F_{q_{0}, 0}\left(\mathrm{cr}_{i}\right)$ with $1 \leq i \leq N_{-}$and choosing a system of mutually non-intersecting paths $\gamma_{i}$ connecting $\mathbf{u}_{i}$ and $\mathbf{u}_{0}$, we have finite Lefschetz thimbles $\Gamma_{i} \cong D^{n}$ emanating from the critical point $\mathrm{cr}_{i}$ and fibred over the path $\gamma_{i}$; then $\Gamma_{1}, \ldots, \Gamma_{N_{-}}$ define relative cycles in the pair $\left(\mathcal{Y}_{q_{0}}^{\mathrm{sm}}, F_{q_{0}, 0}^{-1}\left(\mathbf{u}_{0}\right)\right)$ and form a basis of Lef ${ }_{q_{0}}^{\mathrm{sm}}$. We choose $\eta_{0}>0$ big enough so that $\Gamma_{i}$ are contained in $A_{q_{0}, 0}\left(\eta_{0}\right)$ and that the conditions in (1) are satisfied. The critical points $\mathrm{cr}_{i}$ belong to convergent critical branches, and as such, vary continuously in a neighbourhood of $\left(q_{0}, 0\right)$ in $\mathcal{M}_{-}$; the path $\gamma_{i}$ can be also continuously deformed to a family of paths $\gamma_{i}(q, t)$ connecting the critical value $F_{q, t}\left(\mathrm{cr}_{i}\right)$ and $\mathbf{u}_{0}$. Due to the compactness, the finite thimble $\Gamma_{i}$ can be continuously deformed ${ }^{22}$ to a finite thimble $\Gamma_{i}(q, t) \subset A_{q, t}\left(\eta_{0}\right)$ for $F_{q, t}$ fibred over $\gamma_{i}(q, t)$ when $(q, t)$ is sufficiently close to $\left(q_{0}, 0\right) ; \Gamma_{i}(q, t)$ gives a relative cycle of $\left(A_{q, t}\left(\eta_{0}\right), F_{q, t}^{-1}\left(\mathbf{u}_{0}\right) \cap A_{q, t}\left(\eta_{0}\right)\right)$. As relative cycles in $\left(\mathcal{Y}_{\mathbf{s}_{t}(q)}, F_{\mathbf{s}_{t}(q)}^{-1}\left(\mathbf{u}_{0}\right)\right), \Gamma_{1}\left(\mathbf{s}_{t}(q)\right), \ldots, \Gamma_{N_{-}}\left(\mathbf{s}_{t}(q)\right)$ form part of a basis of the $n$th relative homology (corresponding to the convergent critical points) when $t>0$ is sufficiently small and $q$ is sufficiently close to $q_{0}$. Thus $\iota_{q_{0}}$ sends a basis to part of a basis, and is injective.

(5) That $\iota$ maps $\Gamma_{\mathbb{R}}$ to $\Gamma_{\mathbb{R}}$ along $\mathbb{D}_{\mathrm{sm}}^{\times} \cap \mathcal{M}_{\mathbb{R}}^{\mathrm{sm}}$. The positive real Lefschetz thimble $\Gamma_{\mathbb{R}}$ (see Section 7.3) defines a global section of Lef ${ }^{\mathrm{sm}}$ over $\mathbb{D}_{\mathrm{sm}}^{\times} \cap \mathcal{M}_{\mathbb{R}}^{\mathrm{sm}}$ and a global section of $\mathbf{s}_{>0}^{-1}$ Lef over $(0,1] \times\left(\mathbb{D}_{\mathrm{sm}}^{\times} \cap \mathcal{M}_{\mathbb{R}}^{\mathrm{sm}}\right)$. It is obvious from the construction that the map $\iota_{q_{0}}$ with $q_{0} \in \mathbb{D}_{\mathrm{sm}}^{\times} \cap \mathcal{M}_{\mathbb{R}}^{\mathrm{sm}}$ sends $\Gamma_{\mathbb{R}}\left(q_{0}\right)$ to the germ in $\left(i^{-1} j_{*} \mathbf{s}_{>0}^{-1} \text { Lef }\right)_{q_{0}}$ given by $\left\{\Gamma_{\mathbb{R}}\left(\mathbf{s}_{t}(q)\right)\right\}_{t>0, q}$.

\subsection{Functoriality}

Let $\mathcal{V}_{0}$ be an open neighbourhood of $\mathcal{C} \backslash\left\{0_{+}, 0_{-}\right\}$as in Section 7.4.1. We consider the open dense subset $\mathcal{V}_{0}^{\text {ss }}$ of $\mathcal{V}_{0}$ :

$$
\mathcal{V}_{0}^{\text {ss }}:=\left\{q \in \mathcal{V}_{0} \cap \mathcal{M}^{\times}: F_{q}=\left.F\right|_{p^{-1}(q)} \text { has only non-degenerate critical points }\right\} .
$$

Take $q_{0} \in \mathcal{V}_{0}^{\text {ss }}$ and choose an admissible phase $\phi$ for the critical values of $F_{q_{0}}$. By composing the formal decomposition of the analytified Brieskorn module in Proposition 6.10 and (the nonequivariant version of) the mirror isomorphism in Theorem 4.34, we get formal decompositions of the pulled-back quantum D-modules over an analytic open neighbourhood $B$ of $q_{0}$ :

$$
\left.\operatorname{mir}_{ \pm}^{*} \overline{\operatorname{QDM}^{\mathrm{an}}}\left(\mathfrak{X}_{ \pm}\right)\right|_{B} \cong \bigoplus_{i=1}^{N_{ \pm}}\left(\mathcal{O}_{B} \llbracket z \rrbracket, d+d\left(\mathbf{u}_{i} / z\right), P_{\mathrm{std}}\right)
$$

where $N_{ \pm}=\operatorname{dim} H_{\mathrm{CR}}^{*}\left(\mathfrak{X}_{ \pm}\right)$and $\mathbf{u}_{i}$ are relative critical values of $F$. By Proposition 6.5, by shrinking $B$ if necessary, we have analytic lifts of these formal decompositions over $B \times I_{\phi}$, where $I_{\phi}=\left\{\left(r, e^{\mathbf{i} \theta}\right) \in \widetilde{\mathbb{C}}:|\theta-\phi|<\frac{\pi}{2}+\epsilon\right\}$ (for some small $\epsilon>0$ ):

$$
\left.\pi^{*} \operatorname{mir}_{ \pm}^{*} \operatorname{QDM}^{\mathrm{an}}\left(\mathfrak{X}_{ \pm}\right)\right|_{B \times I_{\phi}} \cong \bigoplus_{i=1}^{N_{ \pm}}\left(\mathcal{A}_{B \times I_{\phi}}, d+d\left(\mathbf{u}_{i} / z\right)\right) \text {, }
$$

where $\pi: \mathcal{M}^{\times} \times \widetilde{\mathbb{C}} \rightarrow \mathcal{M}^{\times} \times \mathbb{C}$ denotes the oriented real blowup along $\mathcal{M} \times\{0\}$. Summands of this sectorial decomposition are indexed by relative critical points of $F$; those for $\mathfrak{X}_{+}$are indexed

\footnotetext{
${ }^{22}$ Away from the critical point, we use a symplectic connection to deform the thimble; around the critical point we use a family version of the Morse lemma.
} 
by all critical points and those for $\mathfrak{X}_{-}$are indexed by the subset of convergent critical points. Combining these two decompositions, we get a sectorial decomposition:

$$
\left.\left.\pi^{*} \operatorname{mir}_{+}^{*} \mathrm{QDM}^{\mathrm{an}}\left(\mathfrak{X}_{+}\right)\right|_{B \times I_{\phi}} \cong \pi^{*} \operatorname{mir}_{-}^{*} \mathrm{QDM}^{\mathrm{an}}\left(\mathfrak{X}_{-}\right)\right|_{B \times I_{\phi}} \oplus \mathcal{A}_{B \times I_{\phi}}^{\oplus\left(N_{+}-N_{-}\right)} .
$$

This gives an analytic lift of the formal decomposition in Theorem 5.16. We consider the inclusion

$$
\left.\left.\pi^{*} \operatorname{mir}_{-}^{*} \mathrm{QDM}^{\mathrm{an}}\left(\mathfrak{X}_{-}\right)\right|_{B \times I_{\phi}} \hookrightarrow \pi^{*} \operatorname{mir}_{+}^{*} \mathrm{QDM}^{\mathrm{an}}\left(\mathfrak{X}_{+}\right)\right|_{B \times I_{\phi}}
$$

induced by (7.15). Note that the maps (7.15), (7.16) are associated with a semisimple point $q_{0}$ and an admissible direction $e^{\mathbf{i} \phi}$.

Theorem 7.25. Let $\varphi: \mathfrak{X}_{+} \rightarrow \mathfrak{X}_{-}$be a toric birational morphism as in Section 7.1. For each point $q_{*} \in \mathcal{V}_{-, \mathbb{R}}^{\mathrm{sm}, \mathrm{ss}} \cap \mathbb{D}_{\mathrm{sm}}^{\times \circ} \subset \mathcal{M}_{\mathbb{R}}^{\mathrm{sm}}$, there exists a contractible open neighbourhood $W_{*}$ of $q_{*}$ in $\mathcal{V}_{-, \mathbb{R}}^{\mathrm{sm}, \mathrm{ss}} \cap \mathbb{D}_{\mathrm{sm}}^{\times \circ}$ and positive numbers $t_{*} \in(0,1), \alpha_{0} \in\left(0, \frac{\pi}{2 J}\right)$ such that the following holds. For each $q_{0} \in \bigcup_{0<t<t_{*}} \mathbf{s}_{t}\left(W_{*}\right) \cap \mathcal{V}_{0}^{\text {ss }}$ and each $\phi \in\left(-\alpha_{0}, \alpha_{0}\right)$ that is admissible for the critical values of $F_{q_{0}}$, the inclusion (7.16) associated with $q_{0}$ and $e^{\mathbf{i} \phi}$ is induced by the pull-back in K-theory via the $\widehat{\Gamma}$-integral structure, i.e., it sends $\mathfrak{s}_{V}$ to $\mathfrak{s}_{\varphi^{*} V}$ for $V \in K\left(\mathfrak{X}_{-}\right)$.

Recall from Section 7.3.2 that $\mathcal{V}_{-, \mathbb{R}}^{\text {sm,ss }}$ is the intersection of the semisimple locus $\mathcal{V}_{-}^{\text {ss }}$ of $\mathcal{V}_{-}$and the real positive locus $\mathcal{M}_{\mathbb{R}}^{\mathrm{sm}}$ of $\mathcal{M}^{\mathrm{sm}}$. Also, $\mathbb{D}_{\mathrm{sm}}^{\times \circ}$ denotes the interior of $\mathbb{D}_{\mathrm{sm}}^{\times}=\mathbb{D}_{\mathrm{sm}} \cap \mathcal{M}^{\mathrm{sm}, \times}$ as a subset of $\mathcal{M}^{\mathrm{sm}}$. Recall that $\mathbf{s}_{t}$ is the sliding map from Definition 7.14 and $J \geq 1$ is the natural number in Proposition 7.2. Note that $\bigcup_{0<t<t_{*}} \mathrm{~s}_{t}\left(W_{*}\right) \cap \mathcal{V}_{0}^{\text {ss }}$ is non-empty because $\mathcal{V}_{0}^{\text {ss }} \cap \mathcal{M}_{\mathbb{R}}$ is open dense in $\mathcal{V}_{0} \cap \mathcal{M}_{\mathbb{R}}$ and $\bigcup_{0<t<t_{*}} \mathbf{s}_{t}\left(W_{*}\right)$ is an open subset of $\mathcal{V}_{0} \cap \mathcal{M}_{\mathbb{R}}$ by Lemma 7.15(2).

Remark 7.26. As we explained in Remark 7.7, we have a standard identification between $K$ classes of $\mathfrak{X}_{+}\left(\right.$resp. $\left.\mathfrak{X}_{-}\right)$and flat sections over $\left(\mathcal{M}_{\mathbb{R}} \cap \mathcal{V}_{+}\right) \times \mathbb{R}_{>0}\left(\operatorname{resp} .\left(\mathcal{M}_{\mathbb{R}}^{\text {sm }} \cap \mathcal{V}_{-}\right) \times \mathbb{R}_{>0}\right)$. We use these identifications in the above theorem; we also use the analytic continuation along the sliding homotopy $\mathbf{s}_{t}$ for $\mathfrak{X}_{-}$.

Remark 7.27. By the duality of the analytic lift discussed in Remark 6.7, if the inclusion (7.16) corresponds to the pull-back $\varphi^{*}: K\left(\mathfrak{X}_{-}\right) \rightarrow K\left(\mathfrak{X}_{+}\right)$in $K$-theory, the projection associated with the opposite direction $-e^{\mathbf{i} \phi}=e^{\mathbf{i}(\phi+\pi)}$

$$
\left.\left.\pi^{*} \operatorname{mir}_{+}^{*} \mathrm{QDM}^{\mathrm{an}}\left(\mathfrak{X}_{+}\right)\right|_{B \times I_{\phi+\pi}} \rightarrow \pi^{*} \operatorname{mir}_{-}^{*} \mathrm{QDM}^{\mathrm{an}}\left(\mathfrak{X}_{-}\right)\right|_{B \times I_{\phi+\pi}}
$$

corresponds to the push-forward $\varphi_{*}: K\left(\mathfrak{X}_{+}\right) \rightarrow K\left(\mathfrak{X}_{-}\right)$in $K$-theory.

Lemma 7.28. Let $q_{*}$ be a point in $\mathcal{M}_{\mathbb{R}}^{\mathrm{sm}} \cap \mathbb{D}_{\mathrm{sm}}^{\times}$. We have a commutative diagram

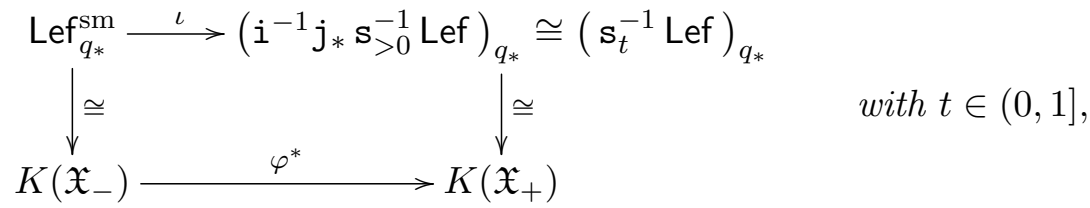

where the vertical arrows are induced by the isomorphisms in Theorems 7.5 and 7.12 , the top horizontal arrow is the inclusion in Theorem 7.22 . 
Proof. Note that the left vertical arrow is induced, via the $\widehat{\Gamma}$-integral structure, by the isomorphism of local systems over $\mathbb{D}_{\mathrm{sm}}^{\times}$:

$$
\left.\operatorname{Lef}^{\mathrm{sm}} \otimes \mathbb{C}\right|_{\mathbb{D}_{\mathrm{sm}}^{\times}} \cong\left(\text { mir. }\left._{-}^{*} \operatorname{QDM}^{\mathrm{an}}\left(\mathfrak{X}_{-}\right)\right|_{\mathbb{D}_{\mathrm{sm}}^{\times} \times\{1\}}\right)^{\nabla}
$$

sending a class $[\Gamma] \in H_{n}\left(\mathcal{Y}_{q},\left\{\Re\left(F_{q}\right) \gg 0\right\}\right)$ to a flat section $s_{\Gamma}$ of $\left.\operatorname{mir}_{-}^{*} \operatorname{QDM}^{\text {an }}\left(\mathfrak{X}_{-}\right)\right|_{\mathbb{D}_{\mathrm{sm}}^{\times} \times\{1\}}$ satisfying

$$
\frac{1}{(2 \pi)^{n / 2}} \int_{\Gamma} e^{-F_{q}} \Omega_{q,-1}=P\left(\operatorname{Mir}(\Omega), s_{\Gamma}\right)
$$

for every local section $\Omega_{q, z}$ of $\operatorname{Bri}^{\mathrm{sm}}(F)$. Here $(\cdots)^{\nabla}$ denotes the local system defined by the kernel of $\nabla$. Also, the right vertical arrow in (7.17) is induced by the isomorphism of local systems over $\operatorname{Im}\left(\mathbf{s}_{>0}\right)$ :

$$
\text { Lef }\left.\otimes \mathbb{C}\right|_{\operatorname{Im}\left(\mathbf{s}_{>0}\right)} \cong\left(\left.\operatorname{mir}_{+}^{*} \operatorname{QDM}^{\mathrm{an}}\left(\mathfrak{X}_{+}\right)\right|_{\operatorname{Im}\left(\mathbf{s}_{>0}\right) \times\{1\}}\right)^{\nabla},
$$

which is defined similarly. We conclude the lemma by studying the monodromy of these local systems. Let $\mathbb{L}^{\prime}=\operatorname{Ker}\left(\mathbb{Z}^{S_{-}} \rightarrow \mathbf{N}\right)$ be as in Section 6.3.3. The inclusion $\mathbb{D}_{\mathrm{sm}}^{\times} \subset \mathcal{M}^{\mathrm{sm}, \times}=$ $\left(\mathbb{L}^{\prime}\right)^{\star} \otimes \mathbb{C}^{\times}$induces an isomorphism $\pi_{1}\left(\mathbb{D}_{\mathrm{sm}}^{\times}\right) \cong\left(\mathbb{L}^{\prime}\right)^{\star}$; an element $\xi \in\left(\mathbb{L}^{\prime}\right)^{\star}$ corresponds to the loop $[0,2 \pi] \ni \theta \mapsto e^{\mathrm{i} \theta \xi} \cdot q_{*}$ based at $q_{*} \in \mathbb{D}_{\mathrm{sm}}^{\times}$. We claim that the monodromy of Lef ${ }^{\mathrm{sm}}$ around the loop $e^{\mathbf{i} \theta \xi} \cdot q_{*}$ corresponds to tensoring by $L_{\xi}^{-1}$ in $K\left(\mathfrak{X}_{-}\right)$on the $\widehat{\Gamma}$-integral structure under the isomorphism (7.18), where $L_{\xi}$ denotes the orbi-line bundle as in Section 3.1.2. We also claim that the monodromy of Lef around $\mathrm{s}_{t}\left(e^{\mathrm{i} \theta \xi} \cdot q_{*}\right)=\left(e^{\mathbf{i} \theta \xi} \cdot q_{*}, t\right)$ corresponds to tensoring by $\varphi^{*} L_{\xi}^{-1}$ in $K\left(\mathfrak{X}_{+}\right)$under the isomorphism (7.19). These two claims prove the lemma. In fact, since the map $\iota$ is monodromy-equivariant, the composition

$$
K\left(\mathfrak{X}_{-}\right) \cong \operatorname{Lef}_{q_{*}}^{\mathrm{sm}} \stackrel{\iota}{\longrightarrow}\left(\mathrm{s}_{t}^{-1} \text { Lef }\right)_{q_{*}} \cong K\left(\mathfrak{X}_{+}\right)
$$

identifies tensoring by $L_{\xi}$ with tensoring by $\varphi^{*} L_{\xi}$; also this map (7.20) sends the structure sheaf to the structure sheaf since $\iota$ sends the positive real Lefschetz thimble $\Gamma_{\mathbb{R}}\left(q_{*}\right)$ to the positive-real one $\Gamma_{\mathbb{R}}\left(\mathbf{s}_{t}\left(q_{*}\right)\right.$ ) (see Theorem 7.22 ); since $K\left(\mathfrak{X}_{-}\right)$is generated by line bundles [16], we conclude that the map (7.20) equals $\varphi^{*}$.

It remains to prove the above two claims. Let $[\xi]$ denote the class in $\operatorname{Pic}\left(\mathfrak{X}_{-}\right)$of $L_{\xi}$. By [62, (61)], the mirror map satisfies

$$
\operatorname{mir}_{-}\left(e^{2 \pi \mathbf{i} \xi} \cdot q_{*}\right)=g(-[\xi]) \operatorname{mir}_{-}\left(q_{*}\right)
$$

(see Section 2.2 for $g(-[\xi]))$ and hence the flat section $\mathfrak{s}_{V}\left(\operatorname{mir}_{-}\left(q_{*}\right), z=1\right.$ ) is analytically continued, along the path $\theta \mapsto e^{\mathbf{i} \theta \xi} \cdot q_{*}$, to $\mathfrak{s}_{V}\left(g(-[\xi]) \operatorname{mir}_{-}\left(q_{*}\right), 1\right)$, which is identified with $d g(-[\xi])^{-1} \mathfrak{s}_{V}\left(g(-[\xi]) \operatorname{mir}_{-}\left(q_{*}\right), 1\right)=\mathfrak{s}_{V \otimes L_{\xi}^{-1}}\left(\operatorname{mir}_{-}\left(q_{*}\right), 1\right)$ by the Galois action in Section 2.3; here we used the formula (2.12). This proves the first claim. To prove the second claim, it suffices to show that the homotopy class $\hat{\xi} \in \mathbb{L}^{\star} \cong \pi_{1}\left(\mathcal{M}^{\times}\right)$of the loop $\mathrm{s}_{t}\left(e^{\mathbf{i} \theta \xi} \cdot q_{*}\right)$ defines a line bundle $L_{\hat{\xi}}$ isomorphic to $\varphi^{*} L_{\xi}$. Observe that we have a direct sum decomposition

$$
\mathbb{L}=\mathbb{L}^{\prime} \oplus \mathbb{Z} \delta_{\hat{b}}^{\boldsymbol{\Sigma}_{-}},
$$

where $\delta_{\hat{b}}^{\boldsymbol{\Sigma}_{-}}=e_{\hat{b}}-\Psi_{-}(\hat{b})=-\mathbf{w}$ is an element introduced in Section 3.3 (see also Section 7.1) which corresponds to the co-ordinate $t=t_{\hat{b}}$. The class $\hat{\xi} \in \mathbb{L}^{\star}$ is a unique lift of $\xi \in\left(\mathbb{L}^{\prime}\right)^{\star}$ such that $\hat{\xi} \cdot \delta_{\hat{b}}^{\boldsymbol{\Sigma}_{-}}=0$. The map (7.1) inducing the birational morphism $\varphi: \mathfrak{X}_{+} \rightarrow \mathfrak{X}_{-}$is equivariant with respect to the homomorphism $\mathbb{L} \otimes \mathbb{C}^{\times} \rightarrow \mathbb{L}^{\prime} \otimes \mathbb{C}^{\times}, \exp (\lambda) \mapsto \exp \left(\lambda+\left(D_{\hat{b}} \cdot \lambda\right) \mathbf{w}\right.$ ) (where $\lambda \in \mathbb{L}_{\mathbb{C}}$ ), which is dual to the above lift $\left(\mathbb{L}^{\prime}\right)^{\star} \rightarrow \mathbb{L}^{\star}, \xi \mapsto \hat{\xi}$. In view of the definition of $L_{\hat{\xi}}$ in Section 3.1.2, we see that $L_{\hat{\xi}} \cong \varphi^{*} L_{\xi}$. The lemma is proved. 
We show that $\alpha_{0}$ appearing in Proposition 7.8 can be chosen independently of $q_{0} \in \mathcal{V}_{+, \mathbb{R}}^{\text {ss }}$ if $q_{0}$ is close to a given point $q_{*}$ in $\mathcal{V}_{-, \mathbb{R}}^{\mathrm{sm}, \mathrm{ss}} \cap \mathbb{D}_{\mathrm{sm}}^{\times \circ}$.

Lemma 7.29. Let $q_{*}$ be a point in $\mathcal{V}_{-, \mathbb{R}}^{\mathrm{sm}, \mathrm{ss}} \cap \mathbb{D}_{\mathrm{sm}}^{\times \circ}$. There exist a contractible open neighbourhood $W_{*}$ of $q_{*}$ in $\mathcal{V}_{-, \mathbb{R}}^{\mathrm{sm}, \mathrm{ss}} \cap \mathbb{D}_{\mathrm{sm}}^{\times 0}$ and positive numbers $t_{*} \in(0,1), \alpha_{0} \in\left(0, \frac{\pi}{2 J}\right)$ such that the conclusion of Proposition 7.8 holds for all $q_{0} \in \bigcup_{0<t<t_{*}} \mathbf{s}_{t}\left(W_{*}\right) \cap \mathcal{V}_{0}^{\mathrm{ss}} \subset \mathcal{V}_{+, \mathbb{R}}^{\mathrm{ss}}$ with this same $\alpha_{0}$.

Proof. Let $\mathrm{cr}_{1}(q), \ldots, \mathrm{cr}_{N_{-}}(q)$ be branches of "convergent" critical points of $F_{q}$ contained in $\mathcal{B}_{-}$ and defined in a neighbourhood of $q=q_{*}$ in $\mathcal{V}_{-}^{\text {ss }}$. We assume that $\operatorname{cr}_{1}\left(q_{*}\right)=\operatorname{cr}_{\mathbb{R}}\left(q_{*}\right)$ and write $\mathbf{u}_{i}(q)=F_{q}\left(\operatorname{cr}_{i}(q)\right)$ as before. We may also assume that $\mathbf{u}_{i}\left(q_{*}\right) \in \mathbf{u}_{1}\left(q_{*}\right)+\mathbb{R}_{\geq 0}$ if and only if $1 \leq i \leq k$. Choose $\alpha_{0} \in\left(0, \frac{\pi}{2 J}\right)$ so that the closed sector $I_{*}:=\mathbf{u}_{1}\left(q_{*}\right)+\left\{r e^{\mathbf{i} \theta}: r \geq 0,|\theta| \leq \alpha_{0}\right\}$ with vertex at $\mathbf{u}_{1}\left(q_{*}\right)$ does not contain critical values other than $\mathbf{u}_{1}\left(q_{*}\right), \ldots, \mathbf{u}_{k}\left(q_{*}\right)$, see Fig. 12 . We can find a contractible open neighbourhood $W_{*}$ of $q_{*}$ in $\mathcal{V}_{-, \mathbb{R}}^{\mathrm{sm}} \cap \mathbb{D}_{\mathrm{sm}}^{\times \circ}, 0<t_{*}<1$, and $\epsilon>0$ such that for all $q \in \bigcup_{0 \leq t<t_{*}} \mathbf{s}_{t}\left(W_{*}\right), \mathbf{u}_{i}(q) \in-\epsilon+I_{*}$ if and only if $1 \leq i \leq k$. Note that $W_{*} \subset \mathcal{V}_{-}^{\mathrm{sm}, \mathrm{ss}} \subset \mathcal{M}^{\mathrm{sm}, \mathrm{nd}}$ and $\bigcup_{0<t<t_{*}} \mathrm{~s}_{t}\left(W_{*}\right) \subset \mathcal{V}_{0} \subset \mathcal{M}^{\text {nd }}$ by Lemma 7.15 and Remark 7.18.

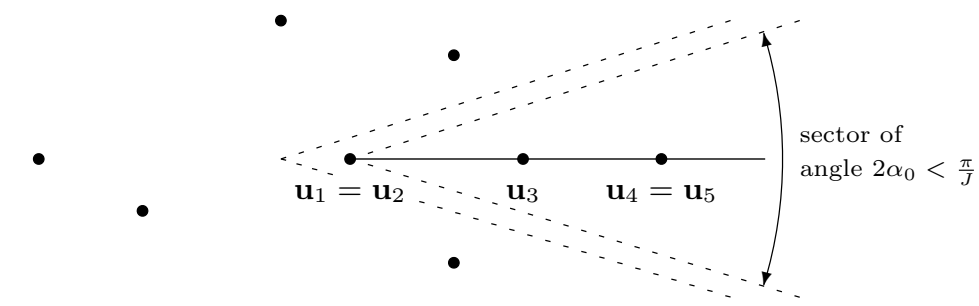

Figure 12. Critical values at $q_{*}$ : only $\mathbf{u}_{1}, \ldots, \mathbf{u}_{k}$ (critical values on the half-line $\mathbf{u}_{1}+\mathbb{R}_{\geq 0}$ ) lie in the sector $-\alpha_{0} \leq \arg \left(\mathbf{u}-\mathbf{u}_{1}\right) \leq \alpha_{0}(k=5)$.

We also have divergent critical points over $\bigcup_{0<t<t_{*}} \mathrm{~s}_{t}\left(W_{*}\right)$; let $\operatorname{cr}_{j}(q), j=N_{-}+1, \ldots, N_{+}$ denote divergent critical branches over $\bigcup_{0<t<t_{*}} \mathbf{s}_{t}\left(W_{*}\right)$ (they can be ramified and may not be single-valued). By taking smaller $t_{*}>0$ if necessary, by Lemma 7.15(3), we may assume that the divergent critical values $\mathbf{u}_{j}(q)=F_{q}\left(\mathrm{cr}_{j}(q)\right), N_{-}+1 \leq j \leq N_{+}$do not lie in the sector $-\epsilon+I_{*}$ as long as $q \in \bigcup_{0<t<t_{*}} \mathbf{s}_{t}\left(W_{*}\right)$.

Let $\Gamma_{i}^{\phi}(q)$ denote the Lefschetz thimble (6.12) of $F_{q}$ associated with the critical point $\mathrm{cr}_{i}(q)$ and the phase $\phi$. Here $q$ can be either in $W_{*}$ or in $\bigcup_{0<t<t_{*}} \mathbf{s}_{t}\left(W_{*}\right)-$ we have $1 \leq i \leq N_{-}$in the former case and $1 \leq i \leq N_{+}$in the latter case. In view of the proof of Proposition 7.8, it suffices to show that the relative homology class

$$
\left[\Gamma_{1}^{\pi+\phi}(q)\right] \in H_{n}\left(\mathcal{Y}_{q},\left\{\Re\left(F_{q}\right) \gg 0\right\} ; \mathbb{Z}\right)
$$

represented by the thimble $\Gamma_{1}^{\pi+\phi}(q)$ is constant as $(q, \phi)$ varies in $\underset{0<t<t_{*}}{\bigcup} \mathbf{s}_{t}\left(W_{*}\right) \times\left(-\alpha_{0}, \alpha_{0}\right)$. The homology class $\left[\Gamma_{1}^{\pi+\phi}(q)\right]$ can jump by the Picard-Lefschetz transformation as $(q, \phi)$ varies (see, e.g., [7, Chapter I]). By our choice of $W_{*}, t_{*}$ and $\alpha_{0}$, it suffices to check that the intersection numbers of vanishing cycles at $\operatorname{cr}_{1}(q)$ and $\operatorname{cr}_{i}(q)$ (with $2 \leq i \leq k$ ) are zero at some $q \in \bigcup_{0<t<t_{*}} \mathbf{s}_{t}\left(W_{*}\right)$; here we consider vanishing cycles associated with paths from $\mathbf{u}_{i}(q)$ to a base point $\mathbf{u}_{0} \gg 0$ inside the sector $-\epsilon+I_{*}$. At $(q, \phi)=\left(q_{*}, 0\right)$, the Lefschetz thimble $\Gamma_{1}^{\pi}\left(q_{*}\right)=\Gamma_{\mathbb{R}}\left(q_{*}\right)$ lying over $\mathbf{u}_{1}\left(q_{*}\right)+\mathbb{R}_{\geq 0}$ does not contain the critical points $\operatorname{cr}_{i}\left(q_{*}\right), 2 \leq i \leq k$, and therefore the vanishing cycles at $\operatorname{cr}_{1}\left(q_{*}\right)$ and $\operatorname{cr}_{i}\left(q_{*}\right)$ (with $2 \leq i \leq k$ ) do not intersect. For $1 \leq i \leq k$, choose 
a path $\gamma_{i}$ starting from $\mathbf{u}_{i}\left(q_{*}\right)$ and ending at $\mathbf{u}_{0}$ which avoids other critical points, and let $\Gamma_{i}$ denote the finite Lefschetz thimble (with boundary in $\left.F_{q_{*}}^{-1}\left(\mathbf{u}_{0}\right)\right)$ associated with $\operatorname{cr}_{i}\left(q_{*}\right)$ and the path $\gamma_{i}$. We choose a sufficiently big $\eta_{0}>0$ such that $\Gamma_{i}$ 's are contained in $A_{q_{*}}\left(\eta_{0}\right)$ and that $\partial A_{q_{*}}\left(\eta_{0}\right) \pitchfork F_{q_{*}}^{-1}\left(\mathbf{u}_{0}\right)$. Arguing as in Parts (1), (4) in the proof of Theorem 7.22, we see that $\Gamma_{i}$ can be continuously deformed to a relative cycle $\Gamma_{i}(q)$ of $\left(A_{q}\left(\eta_{0}\right), A_{q}\left(\eta_{0}\right) \cap F_{q}^{-1}\left(\mathbf{u}_{0}\right)\right)$ as $q$ varies in a small neighbourhood of $q_{*}$ in $\mathcal{V}_{-}^{\text {ss }}$. This shows that the vanishing cycles $\partial \Gamma_{1}(q)$ and $\partial \Gamma_{i}(q)$ (with $2 \leq i \leq k$ ) have zero intersection number. The lemma is proved.

Proof of Theorem 7.25. Choose $q_{*} \in \mathcal{V}_{-, \mathbb{R}}^{\mathrm{sm}, \mathrm{ss}} \cap \mathbb{D}_{\mathrm{sm}}^{\times \circ}$. Let $W_{*} \subset \mathcal{V}_{-, \mathbb{R}}^{\mathrm{sm}, \mathrm{ss}} \cap \mathbb{D}_{\mathrm{sm}}^{\times \circ}, t_{*} \in(0,1)$, $\alpha_{0} \in\left(0, \frac{\pi}{2 J}\right)$ as in Lemma 7.29. By taking smaller $\alpha_{0}$ if necessary, we may assume that the conclusion of Proposition 7.13 holds for the same $\alpha_{0}$ at $q_{0}=q_{*}$. As in the proof of Lemma 7.29, let $\mathrm{cr}_{1}(q), \ldots, \mathrm{cr}_{N_{-}}(q)$ denote the convergent critical branches over a neighbourhood of $q_{*}$ in $\mathcal{V}_{-}^{\text {ss }}$ such that $\operatorname{cr}_{1}(q)$ is the conifold point when $q \in \mathcal{M}_{\mathbb{R}} \cup \mathcal{M}_{\mathbb{R}}^{\mathrm{sm}}$. Also, let $\operatorname{cr}_{N_{-}+1}(q), \ldots, \mathrm{cr}_{N_{+}}(q)$ denote divergent critical branches over a neighbourhood of $q_{*}$, defined away from $\mathcal{M}^{\text {sm }}$ (they can be ramified and may not be single-valued). We write $\mathbf{u}_{i}(q)=F_{q}\left(\operatorname{cr}_{i}(q)\right)$ for the corresponding critical value. Let $\phi_{*} \in\left(-\alpha_{0}, \alpha_{0}\right)$ be an admissible phase for $\left\{\mathbf{u}_{1}\left(q_{*}\right), \ldots, \mathbf{u}_{N_{-}}\left(q_{*}\right)\right\}$. Proposition 7.13 gives an analytic decomposition

$$
\widetilde{\Phi}_{\phi_{*}}^{-}:\left.\pi^{*} \operatorname{mir}_{-}^{*} \operatorname{QDM}\left(\mathfrak{X}_{-}\right)\right|_{B^{\prime} \times I_{\phi_{*}}} \cong \bigoplus_{i=1}^{N_{-}}\left(\mathcal{A}_{B^{\prime} \times I_{\phi_{*}}}, d+d\left(\mathbf{u}_{i} / z\right)\right),
$$

where $B^{\prime}$ is an open neighbourhood of $q_{*}$ in $\mathcal{V}_{-}^{\text {ss }}, I_{\phi_{*}}=\left\{\left(r, e^{\mathbf{i} \theta}\right):\left|\theta-\phi_{*}\right|<\frac{\pi}{2}+\epsilon\right\}$ (for some $\epsilon>0)$ and $\pi: \mathcal{V}_{-}^{\text {ss }} \times \widetilde{\mathbb{C}} \rightarrow \mathcal{V}_{-}^{\text {ss }} \times \mathbb{C}$ is the oriented real blowup, with the following property: there exists a basis $V_{1}^{-}, \ldots, V_{N_{-}}^{-}$of $K\left(\mathfrak{X}_{-}\right)$such that $V_{1}^{-}$is the structure sheaf of $\mathfrak{X}_{-}$and that $\widetilde{\Phi}_{\phi}^{-}\left(e^{-\mathbf{u}_{j}(q) / z} s_{j}^{-}\right)=e_{j}$, where $s_{j}^{-}$is the flat section $\mathfrak{s}_{V_{j}^{-}}\left(\operatorname{mir}{ }_{-}(q), z\right)$ associated with $V_{j}^{-}$. Choose $\phi_{1}, \phi_{2} \in\left(\phi_{*}-\frac{\epsilon}{2}, \phi_{*}+\frac{\epsilon}{2}\right.$ ) (where $\epsilon$ is the one appearing in $I_{\phi_{*}}$ ) such that $\phi_{1}<\phi_{2}$ and that all phases in $\left[\phi_{1}, \phi_{2}\right]$ are admissible for $\left\{\mathbf{u}_{1}\left(q_{*}\right), \ldots, \mathbf{u}_{N_{-}}\left(q_{*}\right)\right\}$. By shrinking $W_{*}$ and taking smaller $t_{*}>0$ if necessary, we may assume that

(a) $\bigcup_{0 \leq t<t_{*}} \mathbf{s}_{t}\left(W_{*}\right) \subset B^{\prime}$

(b) $\mathbf{u}_{i}(q)-\mathbf{u}_{j}(q) \notin e^{\mathbf{i} \theta} \mathbb{R}$ for all $q \in \bigcup_{0 \leq t<t_{*}} \mathbf{s}_{t}\left(W_{*}\right), \theta \in\left[\phi_{1}, \phi_{2}\right], i, j \in\left\{1,2, \ldots, N_{-}\right\}$provided that $\mathbf{u}_{i}\left(q_{*}\right) \neq \mathbf{u}_{j}\left(q_{*}\right)$;

(c) $\bigcup_{1 \leq i \leq N_{-}} \mathbf{u}_{i}(q)+\left\{r e^{\mathbf{i} \theta}: r \geq 0,|\theta| \leq \alpha_{0}\right\}$ does not contain divergent critical values $\mathbf{u}_{j}(q)$ with

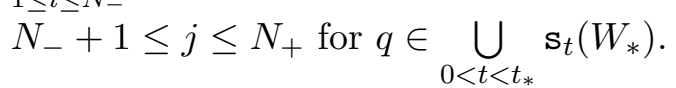

The third point (c) is possible by Lemma 7.15(3) and the fact that $\alpha_{0}<\frac{\pi}{2 J}$.

Take $q_{0} \in \bigcup_{0<t<t_{*}} \mathbf{s}_{t}\left(W_{*}\right) \cap \mathcal{V}_{0}^{\text {ss }}$ and a phase $\phi \in\left(-\alpha_{0}, \alpha_{0}\right)$ which is admissible for $\left\{\mathbf{u}_{1}\left(q_{0}\right), \ldots\right.$, $\left.\mathbf{u}_{N_{+}}\left(q_{0}\right)\right\}$. First we prove the conclusion of the theorem assuming that $\phi$ lies in $\left(\phi_{1}, \phi_{2}\right) \subset$ $\left(-\alpha_{0}, \alpha_{0}\right)$. The discussion for any admissible $\phi \in\left(-\alpha_{0}, \alpha_{0}\right)$ will be postponed until the last paragraph of the proof. Since $\phi \in\left(-\alpha_{0}, \alpha_{0}\right)$, Lemma 7.29 (Proposition 7.8) gives an analytic decomposition $\widetilde{\Phi}_{\phi}^{+}$

$$
\widetilde{\Phi}_{\phi}^{+}:\left.\pi^{*} \operatorname{mir}_{+}^{*} \operatorname{QDM}\left(\mathfrak{X}_{+}\right)\right|_{B \times I_{\phi}} \cong \bigoplus_{i=1}^{N_{+}}\left(\mathcal{A}_{B \times I_{\phi}}, d+d\left(\mathbf{u}_{i} / z\right)\right),
$$

where $B$ is a neighbourhood of $q_{0}$ in $\mathcal{V}_{-}^{\text {ss }}$ and $I_{\phi}$ is a sector of the form $\left\{\left(r, e^{\mathrm{i} \theta}\right) \in \widetilde{\mathbb{C}}:|\theta-\phi|<\frac{\pi}{2}+\right.$ $\delta$ \} (for some $0<\delta \leq \frac{\epsilon}{2}$ ) with the following property: there exists a basis $V_{1}^{+}, \ldots, V_{N_{+}}^{+}$of $K\left(\mathfrak{X}_{+}\right)$ 
such that $V_{1}^{+}$is the structure sheaf of $\mathfrak{X}_{+}$and that $\widetilde{\Phi}_{\phi}^{+}\left(e^{-\mathbf{u}_{j}(q) / z} s_{j}^{+}\right)=e_{j}$, where $s_{j}^{+}$is the flat section $\mathfrak{s}_{V_{j}^{+}}\left(\operatorname{mir}_{+}(q), z\right)$ associated with $V_{j}^{+}$. We may assume that $B \subset B^{\prime}$ by the condition (a) above. We have $I_{\phi} \subset I_{\phi_{*}}$ because $\phi \in\left(\phi_{1}, \phi_{2}\right) \subset\left(\phi_{*}-\frac{\epsilon}{2}, \phi_{*}+\frac{\epsilon}{2}\right)$. Therefore $\widetilde{\Phi}_{\phi_{*}}^{-}$gives the analytic lift associated with the point $q_{0}$ and the phase $\phi$. In particular, the decomposition (7.15) is induced by $\widetilde{\Phi}_{\phi}^{+}$and $\widetilde{\Phi}_{\phi_{*}}^{-}$, and the inclusion (7.16) is induced by the map $\kappa: K\left(\mathfrak{X}_{-}\right) \rightarrow K\left(\mathfrak{X}_{+}\right)$ sending $V_{i}^{-}$to $V_{i}^{+}$for $1 \leq i \leq N_{-}$. It now suffices to show that $V_{i}^{+}=\varphi^{*} V_{i}^{-}$. We already know that this is true for $i=1$.

Recall from the proof of Propositions 7.8, 7.13 that $V_{i}^{+}$(resp. $V_{i}^{-}$) corresponds to the Lefschetz thimble $\Gamma_{i}^{\pi+\phi}\left(q_{0}\right)$ of $F_{q_{0}}$ (resp. the Lefschetz thimble $\Gamma_{i}^{\pi+\phi}\left(q_{*}\right)$ of $\left.F_{q_{*}}\right)$ under the isomorphism in Theorem 7.5 (resp. Theorem 7.12). We claim that the map sending $\Gamma_{i}^{\pi+\phi}\left(q_{*}\right) \in \operatorname{Lef}_{q_{*}}^{\mathrm{sm}}$ to $\Gamma_{i}^{\pi+\phi}\left(q_{0}\right) \in \operatorname{Lef}_{q_{0}}$ coincides with the map $\iota$ from Theorem 7.22. Here we regard $\Gamma_{i}^{\pi+\phi}\left(q_{0}\right)$ as a germ of $\mathbf{i}^{-1} \mathbf{j}_{*} \mathbf{s}_{>0}^{-1}$ Lef at $q_{*}$ by extending it to a Gauss-Manin flat section over $\bigcup_{0<t<t_{*}}^{i} \mathbf{s}_{t}\left(W_{*}\right)$.

Fix a sufficiently large $\mathbf{u}_{0} \gg 0$. Under the isomorphism

$$
H_{n}\left(\mathcal{Y}_{q_{*}},\left\{\Re\left(F_{q_{*}}\right) \gg 0\right\}\right) \cong H_{n}\left(\mathcal{Y}_{q_{*}}, F_{q_{*}}^{-1}\left(\mathbf{u}_{0}\right)\right)
$$

the class of $\Gamma_{i}^{\pi+\phi}\left(q_{*}\right)$ corresponds to the class of a finite Lefschetz thimble fibred over a bent ray as shown in Fig. 13(ii). We move $q_{*}$ along the sliding map. For sufficiently small $t>0$, these finite Lefschetz thimbles can be continuously deformed to finite thimbles for $F_{\mathbf{s}_{t}\left(q_{*}\right)}$ with boundary in $F_{\mathbf{s}_{t}\left(q_{*}\right)}^{-1}\left(\mathbf{u}_{0}\right)$, as discussed in Parts (1) and (4) in the proof of Theorem 7.22. By straightening these paths in the direction $e^{\mathbf{i} \phi}$ again, we see that these relative cycles in $\left(\mathcal{Y}_{\mathbf{s}_{t}\left(q_{*}\right)}, F_{\mathbf{s}_{t}\left(q_{*}\right)}^{-1}\left(\mathbf{u}_{0}\right)\right)$ correspond to the Lefschetz thimbles $\Gamma_{i}^{\pi+\phi}\left(\mathbf{s}_{t}\left(q_{*}\right)\right)$ over the straight ray $\mathbf{u}_{i}\left(\mathbf{s}_{t}\left(q_{*}\right)\right)+e^{\mathbf{i} \phi} \mathbb{R}_{\geq 0}$. Therefore, the map $\iota$ sends $\Gamma_{i}^{\pi+\phi}\left(q_{*}\right)$ to $\Gamma_{i}^{\pi+\phi}\left(\mathbf{s}_{t}\left(q_{*}\right)\right)$ for sufficiently small $t>0$. The assumptions (b), (c) above ensure that the homology class of $\Gamma_{i}^{\pi+\phi}(q)$ stays constant as $q$ varies inside $\bigcup \mathbf{s}_{t}\left(W_{*}\right)$; the Picard-Lefschetz transformation can only arise from the intersection of van$0<t<t_{*}$

ishing cycles at $\operatorname{cr}_{i}(q)$ and $\operatorname{cr}_{j}(q)$ with $i \neq j, \mathbf{u}_{i}\left(q_{*}\right)=\mathbf{u}_{j}\left(q_{*}\right), i, j \in\left\{1, \ldots, N_{-}\right\}$, but these intersection numbers vanish since the vanishing cycles do not intersect at $q_{*}$. This proves the claim.

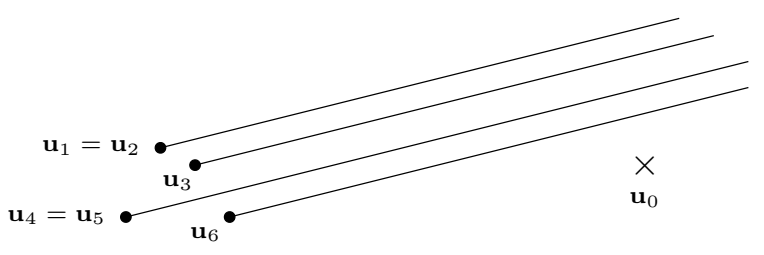

(i)

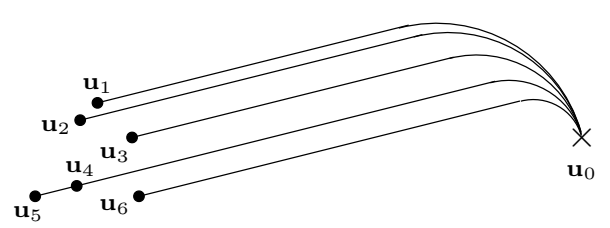

(iii)

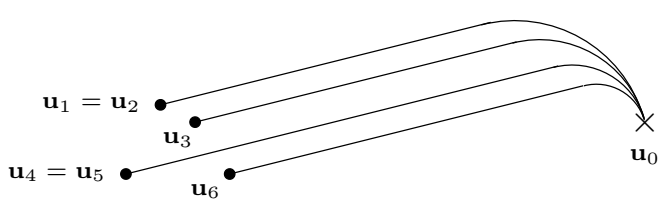

(ii)

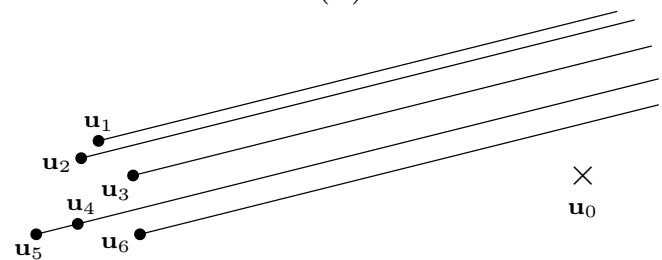

(iv)

Figure 13. Deforming Lefschetz thimbles: (i) semi-infinite Lefschetz thimbles fibred over the ray $\mathbf{u}_{j}\left(q_{*}\right)+$ $\mathbb{R}_{\geq 0} e^{\mathbf{i} \phi}$; (ii) bent rays passing through $\mathbf{u}_{0}$; (iii) moving from $q_{*}$ to a nearby point in $\underset{0<t<t_{*}}{\bigcup} \mathbf{s}_{t}\left(W_{*}\right)$; (iv) straightening the paths again. In (iii) and (iv), the paths from $\mathbf{u}_{4}$ and $\mathbf{u}_{5}$ overlaps, but it does not matter since the relevant vanishing cycles do not intersect. 
The above discussion gives a commutative diagram

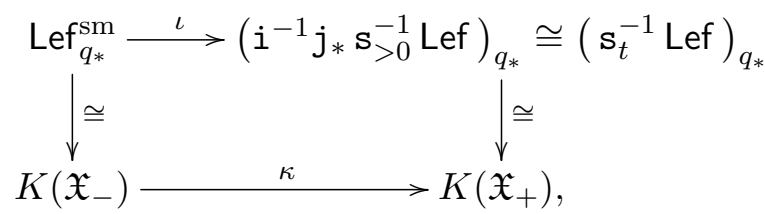

where the vertical arrows are induced by the isomorphisms in Theorems 7.5 and 7.12 , the top horizontal arrow is the inclusion in Theorem 7.22 and the bottom arrow $\kappa$ sends $V_{i}^{-}$to $V_{i}^{+}$for $1 \leq i \leq N_{-}$. Comparing this with the commutative diagram in Lemma 7.28, we conclude that $\kappa=\varphi^{*}$ as required.

Finally, we explain that the conclusion of the theorem holds for every admissible phase $\phi \in\left(-\alpha_{0}, \alpha_{0}\right)$ for $\left\{\mathbf{u}_{1}\left(q_{0}\right), \ldots, \mathbf{u}_{N_{+}}\left(q_{0}\right)\right\}$. As we reviewed in Section 6.2, the analytic decompositions (7.14) for $\mathfrak{X}_{ \pm}$change by mutation as the phase $\phi$ varies; see [44, Sections 2.6, 4.2 and 4.3]. In the case at hand, the analytic decompositions are given by the basis $\left\{s_{j}^{ \pm}\right\}$of flat sections associated with the $K$-classes $\left\{V_{j}^{ \pm}\right\}$; they give rise to an asymptotic basis in the sense of Section 6.2. Their all possible mutations are completely determined by the configuration $\left\{\mathbf{u}_{1}\left(q_{0}\right), \ldots, \mathbf{u}_{N_{ \pm}}\left(q_{0}\right)\right\}$ of the critical values and the Euler pairings $\chi\left(V_{i}^{ \pm}, V_{j}^{ \pm}\right)$. We have $\chi\left(V_{i}^{+}, V_{j}^{+}\right)=\chi\left(V_{i}^{-}, V_{j}^{-}\right)$for $1 \leq i, j \leq N_{-}$since $V_{i}^{+}=\varphi^{*} V_{i}^{-}$and $\varphi$ is birational. The condition (c) above ensures that the basis $\left\{s_{1}^{-}, \ldots, s_{N_{-}}^{-}\right\}$of flat sections for $\mathfrak{X}_{-}$and the part $\left\{s_{1}^{+}, \ldots, s_{N_{-}}^{+}\right\}$ of the basis of flat sections for $\mathfrak{X}_{+}$undergo the same mutation when $\phi$ varies in $\left(-\alpha_{0}, \alpha_{0}\right)$. Hence the relation $V_{i}^{+}=\varphi^{*} V_{i}^{-}$is preserved under mutation. The theorem is proved.

Remark 7.30. We hope that we can analyze general discrepant transformations $\mathfrak{X}_{+} \leftarrow \widehat{\mathfrak{X}} \rightarrow \mathfrak{X}_{-}$ by using the functoriality under blowups (Theorem 7.25) twice. In the case of crepant toric wall crossings, we can see how a Fourier-Mukai transformation (as discussed in $[15,31]$ ) arises from this result, see the slides from [63].

\subsection{Orlov's decomposition and analytic lift}

In the previous section, we have observed that the Lefschetz thimbles associated with 'convergent' critical points (at some point $q_{0} \in \mathcal{V}_{0}^{\text {ss }} \cap \mathcal{M}_{\mathbb{R}}$ and for some phase $\phi$ ) correspond to $K$-classes from $\varphi^{*}\left(K\left(\mathfrak{X}_{-}\right)\right) \subset K\left(\mathfrak{X}_{+}\right)$. In this section, we see that the remaining 'divergent' critical points correspond to $K$-classes supported on the exceptional divisor of $\varphi: \mathfrak{X}_{+} \rightarrow \mathfrak{X}_{-}$. We will see the correspondence between Orlov's semiorthogonal decomposition of the derived category $D^{b}\left(\mathfrak{X}_{+}\right)$ and the analytic decomposition (7.15) of the quantum D-module of $\mathfrak{X}_{+}$.

\subsubsection{Decomposition of the relative homology mirror to Orlov's decomposition}

Recall from Lemma 7.15(3) that convergent critical values over $\mathrm{s}_{t}\left(\mathbb{D}_{\mathrm{sm}}\right)$ are contained in $B_{\rho_{2}}(0)$ and divergent critical values over $\mathbf{s}_{t}\left(\mathbb{D}_{\mathrm{sm}}\right)$ are contained in the $J$ 'satellite' discs $\mathfrak{B}_{k}(t):=t^{-1 / J}$. $B_{\rho_{1}}\left(3 \rho_{0} e^{(-2 k-1) \pi \mathbf{i} / J}\right), k \in \mathbb{Z} / J \mathbb{Z}$ (see Fig. 10). We choose $t_{1}>0$ small enough so that $\overline{B_{\rho_{2}}(0)}+$ $\mathbb{R}_{\geq 0}$ does not intersect any other satellite discs $\overline{\mathfrak{B}_{k}(t)}$ when $t$ is real and $0<t \leq t_{1}$. Then, if $|\phi|$ is sufficiently small, we have that

$$
\mathcal{S}_{\text {conv }}:=\overline{B_{\rho_{2}}(0)}+\mathbb{R}_{\geq 0} e^{\mathbf{i} \phi}
$$

does not intersect with any $\overline{\mathfrak{B}_{k}(t)}$ when $0<t \leq t_{1}$. We choose a number $h \in\{0,1,2, \ldots, J\}$ and let $\mathcal{S}_{k} \subset \mathbb{C}, k=-h,-h+1, \ldots, J-h-1$ be mutually disjoint strip regions as in Fig. 14; each $\mathcal{S}_{k}$ is a closed region disjoint from $\mathcal{S}_{\text {conv }}$, emanating from $\overline{\mathfrak{B}_{k}(t)}$, going around the origin by the angle $(2 k-1) \pi / J+\phi$ anticlockwise, and extending straight toward the direction $e^{\mathbf{i} \phi}$ near 


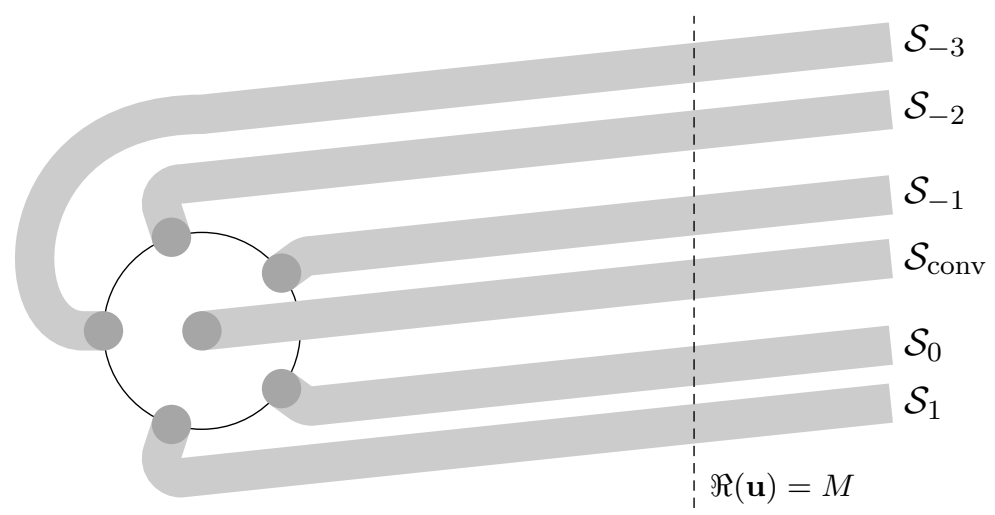

Figure 14. Strip regions $\mathcal{S}_{\text {conv }}$ and $\mathcal{S}_{k}$. In this picture, $J=5, h=3$ and $\phi=\frac{\pi}{30}$.

the end. These regions $\mathcal{S}_{\text {conv }}, \mathcal{S}_{k}$ depend on $t, \phi$ and are defined when $0<t \leq t_{1}$ and $|\phi|$ is sufficiently small.

For sufficiently large $M \gg 0, \bigcup_{k=-h}^{J-h-1} \mathcal{S}_{k} \cup \mathcal{S}_{\text {conv }} \cup\{\Re(\mathbf{u}) \geq M\}$ is a strong deformation retract of $\mathbb{C}$. The fibration given by $F_{q, t}$ is smoothly trivial outside this region by Corollary 7.21 , and hence we get the decomposition of the relative homology Lef ${ }_{q, t}$ (see (7.10)):

$$
\operatorname{Lef}_{q, t}=\operatorname{Lef}_{q, t}^{(-h)} \oplus \cdots \oplus \operatorname{Lef}_{q, t}^{(-1)} \oplus \operatorname{Lef}_{q, t}^{\operatorname{conv}} \oplus \operatorname{Lef}_{q, t}^{(0)} \oplus \cdots \oplus \operatorname{Lef}_{q, t}^{(J-h-1)}
$$

for $q \in \mathbb{D}_{\mathrm{sm}}^{\times}$and $0<t \leq t_{1}$, where we use the notation as in Sections 7.4.2-7.4.4 and

$$
\begin{aligned}
& \operatorname{Lef}_{q, t}^{\text {conv }}=H_{n}\left(F_{q, t}^{-1}\left(\mathcal{S}_{\text {conv }}\right), F_{q, t}^{-1}\left(\mathcal{S}_{\text {conv }}\right) \cap\left\{x: \Re\left(F_{q, t}(x)\right) \geq M\right\} ; \mathbb{Z}\right), \\
& \operatorname{Lef}_{q, t}^{(k)}=H_{n}\left(F_{q, t}^{-1}\left(\mathcal{S}_{k}\right), F_{q, t}^{-1}\left(\mathcal{S}_{k}\right) \cap\left\{x: \Re\left(F_{q, t}(x)\right) \geq M\right\} ; \mathbb{Z}\right) .
\end{aligned}
$$

This decomposition is independent of $\phi$ (with $|\phi|$ sufficiently small) and is preserved by the Gauss-Manin connection. We say that a direct sum decomposition $A=A_{1} \oplus \cdots \oplus A_{m}$ is semiorthogonal with respect to a (not necessarily symmetric) pairing $[\cdot, \cdot)$ on $A$ if $\left[a_{i}, a_{j}\right)=0$ for $a_{i} \in A_{i}, a_{j} \in A_{j}$ whenever $i>j$. Note that the decomposition (7.22) is semiorthogonal with respect to the pairing \# $\left(e^{-\pi \mathbf{i}} \Gamma_{1} \cdot \Gamma_{2}\right)$ discussed in Remark 7.6.

Let $E \subset \mathfrak{X}_{+}$be the exceptional divisor of the morphism $\varphi: \mathfrak{X}_{+} \rightarrow \mathfrak{X}_{-}$; this is the toric divisor corresponding to $\hat{b} \in R_{+}$. Let $Z=\varphi(E)$ denote the image of $E$; this is a toric substack corresponding to the cone $\sigma_{M_{+}}$of $\boldsymbol{\Sigma}_{-}$(see (7.1)). We write $\varphi_{E}: E \rightarrow Z$ for the restriction of $\varphi$ to $E$ and $i_{E}: E \rightarrow \mathfrak{X}_{+}$for the inclusion.

Theorem 7.31. For $q \in \mathbb{D}_{\mathrm{sm}}^{\times} \cap \mathcal{M}_{\mathbb{R}}^{\mathrm{sm}}$ and $0<t \leq t_{1}$, the decomposition (7.22) of the relative homology corresponds to the decomposition of the $K$-group

$$
K\left(\mathfrak{X}_{+}\right)=K(Z)_{-h} \oplus \cdots \oplus K(Z)_{-1} \oplus \varphi^{*} K\left(\mathfrak{X}_{-}\right) \oplus K(Z)_{0} \oplus \cdots \oplus K(Z)_{J-h-1}
$$

under the isomorphism Lef $_{q, t} \cong K\left(\mathfrak{X}_{+}\right)$in Theorem $7.5(1)$, where $K(Z)_{k}$ denotes the subgroup $\mathcal{O}(-k E) \otimes i_{E *} \varphi_{E}^{*} K(Z) \subset K\left(\mathfrak{X}_{+}\right)$.

Remark 7.32. The decomposition (7.23) is semiorthogonal with respect to the Euler pairing by Remark 7.6 and the above theorem. In view of this, we expect that $D^{b}\left(\mathfrak{X}_{+}\right)$admits a semiorthogonal decomposition:

$$
D^{b}\left(\mathfrak{X}_{+}\right)=\left\langle D^{b}(Z)_{-h}, \cdots, D^{b}(Z)_{-1}, \varphi^{*} D^{b}\left(\mathfrak{X}_{-}\right), D^{b}(Z)_{0}, \ldots, D^{b}(Z)_{J-h-1}\right\rangle,
$$


where $D^{b}(Z)_{j}$ denotes the full subcategory of $D^{b}\left(\mathfrak{X}_{+}\right)$which is the image of the functor $\mathcal{O}(-j E) \otimes$ $i_{E *} \varphi_{E}^{*}: D^{b}(Z) \rightarrow D^{b}\left(\mathfrak{X}_{+}\right)$. When $\mathfrak{X}_{+}$is the blowup of a smooth variety $\mathfrak{X}_{-}$along a smooth centre $Z$, this is the semiorthogonal decomposition proved by Orlov [87, Theorem 4.3]; Orlov stated the result for $h=J$, but the other cases $0 \leq h \leq J-1$ follow from this case by using the fact that $D^{b}(Z)_{-h}=\mathbb{S}\left(D^{b}(Z)_{J-h}\right)$, where $\mathbb{S}$ is the Serre functor for $D^{b}\left(\mathfrak{X}_{+}\right)$.

Proof of Theorem 7.31. Observe that the subgroup $\operatorname{Lef}_{q, t}^{\text {conv }} \subset \operatorname{Lef}_{q, t}$ is the image of the map $\iota:$ Lef $_{q}^{\text {sm }} \rightarrow\left(\mathrm{s}_{t}^{-1} \text { Lef }\right)_{q}$ in Theorem 7.22 (e.g., by considering the case $\left.\phi=0\right)$. Hence it corresponds to $\varphi^{*}\left(K\left(\mathfrak{X}_{-}\right)\right) \subset K\left(\mathfrak{X}_{+}\right)$by Lemma 7.28. It suffices to show that Lef ${ }_{q, t}^{(k)}$ corresponds to $K(Z)_{k}$ for $-h \leq k \leq J-h-1$. Let $K^{(k)} \subset K\left(\mathfrak{X}_{+}\right)$denote the subgroup corresponding to Lef $_{q, t}^{(k)} \subset$ Lef $_{q, t}$. It suffices to show that

(a) $K(Z)_{k} \subset K^{(k)}$;

(b) the decomposition (7.23) of the $K$-group holds true.

As discussed, over $\mathbf{s}_{t}\left(\mathbb{D}_{\mathrm{sm}}\right)$, convergent critical values are contained in $B_{\rho_{2}}(0)$ and divergent critical values are contained in the $J$ satellite $\operatorname{discs} \mathfrak{B}_{k}(t), k \in \mathbb{Z} / J \mathbb{Z}$; these statement still hold for a non-zero complex number $t$ with $|t| \leq 1$ by naturally extending the definition of $\mathbf{s}_{t}$ and $\mathfrak{B}_{k}(t)$ to $t \in \mathbb{C}$ (see the proof of Lemma 7.15). Consider the class $\left[\Gamma_{\mathbb{R}}\right] \in$ Lef $_{q, t}$ of the positive real Lefschetz thimble. It corresponds to the class $[\mathcal{O}] \in K\left(\mathfrak{X}_{+}\right)$of the structure sheaf. We study the monodromy action on $\operatorname{Lef}_{q, t}$ along the loop $[0,2 \pi] \ni \theta \mapsto\left(q, e^{\mathbf{i} \theta} t\right)$. The monodromy corresponds to the tensor product by $\mathcal{O}(-E)$ on $K\left(\mathfrak{X}_{+}\right)$; this follows from the same argument as in the proof of Lemma 7.28 and the fact that this loop corresponds to the element of $\mathbb{L}^{\star}$ given by the second projection in the decomposition (7.21) (which equals $D_{\hat{b}} \in \mathbb{L}^{\star}$ ). Under this monodromy, $\left[\Gamma_{\mathbb{R}}\right]$ undergoes the Picard-Lefschetz transformation. Among the satellite discs, only $\mathfrak{B}_{-1}(t)$ passes through the strip region $\mathcal{S}_{\text {conv }}$ as $t$ rotates; here $\mathfrak{B}_{-1}(t)$ becomes $\mathfrak{B}_{-1}\left(e^{2 \pi \mathbf{i}} t\right)=\mathfrak{B}_{0}(t)$ after rotation. Thus by the Picard-Lefschetz formula, the monodromy transform of $\left[\Gamma_{\mathbb{R}}\right]$ equals $\left[\Gamma_{\mathbb{R}}\right]-\alpha_{0}$ for some $\alpha_{0} \in \operatorname{Lef}_{q, t}^{(0)}$. Then we find that $\alpha_{0}$ corresponds to $[\mathcal{O}]-[\mathcal{O}(-E)]=\left[\mathcal{O}_{E}\right] \in$ $K\left(\mathfrak{X}_{+}\right)$and in particular that it is non-zero. Similarly, by considering the monodromy around the inverse loop $\theta \mapsto\left(q, e^{-\mathbf{i} \theta} t\right)$, we find an element $\beta_{1} \in \operatorname{Lef}_{q, t}^{(-1)}$ that corresponds to $[\mathcal{O}(E)]-[\mathcal{O}]=$ $\left[\mathcal{O}_{E}(E)\right] \in K\left(\mathfrak{X}_{+}\right)$. By considering further monodromy actions on $\alpha_{0}, \beta_{1}$, we find elements $\alpha_{i} \in \operatorname{Lef}_{q, t}^{(i)}$ with $0 \leq i \leq J-h-1$ and $\beta_{j} \in \operatorname{Lef}_{q, t}^{(-j)}$ with $1 \leq j \leq h$ such that $\alpha_{i}$ corresponds to $\left[\mathcal{O}_{E}(-i E)\right]$ and that $\beta_{j}$ corresponds to $\left[\mathcal{O}_{E}(j E)\right]$. Hence we have

$$
\left[\mathcal{O}_{E}(-k E)\right]=\mathcal{O}(-k E) \otimes\left[\mathcal{O}_{E}\right] \in K^{(k)}
$$

for $-h \leq k \leq J-h-1$. Note that the decomposition (7.22) is invariant under monodromy in $q \in \mathbb{D}_{\mathrm{sm}}^{\times}$. As discussed in the proof of Lemma 7.28, the monodromy around loops in $\mathbb{D}_{\mathrm{sm}}^{\times}$ corresponds to the tensoring $\varphi^{*} L$ with $L \in \operatorname{Pic}\left(\mathfrak{X}_{-}\right)$. Since $K\left(\mathfrak{X}_{-}\right)$is generated by line bundles, we conclude from (7.24) that $\mathcal{O}(-k E) \otimes \mathcal{O}_{E} \otimes \varphi^{*} V \in K^{(k)}$ for all $V \in K\left(\mathfrak{X}_{-}\right)$. Part (a) follows from the fact that $K(Z)$ is also generated by line bundles and that the natural map $\operatorname{Pic}\left(\mathfrak{X}_{-}\right) \rightarrow \operatorname{Pic}(Z)$ is surjective.

It remains to prove Part (b). In view of part (a) and the decomposition (7.22), it suffices to show that $K\left(\mathfrak{X}_{+}\right)$is generated by the classes of $\mathcal{O}$ and $\mathcal{O}_{E}(-k E),-h \leq k \leq J-h-1$ as a $K\left(\mathfrak{X}_{-}\right)$module, where the module structure is given by $\varphi^{*}: K\left(\mathfrak{X}_{-}\right) \rightarrow K\left(\mathfrak{X}_{+}\right)$. Let $L_{b}=L_{-D_{b}} \in K\left(\mathfrak{X}_{+}\right)$ denote the class of the line bundle associated with $-D_{b} \in \mathbb{L}^{\star}$ for $b \in S$ (see Section 3.1.2 for $\left.L_{-D_{b}}\right) ; 1-L_{b}$ is the class of the structure sheaf of the toric divisor associated with $b$ when $b \in R_{+}$. Similarly, let $L_{b}^{-} \in K\left(\mathfrak{X}_{-}\right)$denote the class of the line bundle associated with $-D_{b} \in\left(\mathbb{L}^{\prime}\right)^{\star}$ for $b \in S_{-}$. We also set $L:=L_{\hat{b}}=[\mathcal{O}(-E)] \in K\left(\mathfrak{X}_{+}\right)$. Recall from the proof of Lemma 7.28 that the splitting $\left(\mathbb{L}^{\prime}\right)^{\star} \rightarrow \mathbb{L}^{\star}, \xi \mapsto \hat{\xi}$ induced by the decomposition (7.21) 
corresponds to the pull-back of line bundles, i.e., $\varphi^{*} L_{\xi} \cong L_{\hat{\xi}}$. Since the splitting $\left(\mathbb{L}^{\prime}\right)^{\star} \rightarrow \mathbb{L}^{\star}$ sends $D_{b}$ to $D_{b}+k_{b} D_{\hat{b}}$ (with $k_{b}=-D_{b} \cdot \delta_{\hat{b}}^{\boldsymbol{\Sigma}_{-}}$as before), we have

$$
\varphi^{*} L_{b}^{-}=L_{b} \cdot L^{k_{b}} \text {. }
$$

On the other hand, since the intersection of the toric divisors corresponding to rays $b \in M_{+}$is empty in $\mathfrak{X}_{+}$, we have the following relation in $K\left(\mathfrak{X}_{+}\right)$(see [16]):

$$
\prod_{b \in M_{+}}\left(1-L_{b}\right)=0
$$

Combining the two relations $(7.25),(7.26)$, we obtain

$$
\prod_{b \in M_{+}}\left(L^{k_{b}}-\varphi^{*} L_{b}^{-}\right)=0
$$

The left-hand side of the relation is a monic polynomial in $L$ of degree $J+1=\sum_{b \in M_{+}} k_{b}$ with coefficients in $K\left(\mathfrak{X}_{-}\right)$whose constant term is an invertible element in $K\left(\mathfrak{X}_{-}\right)$. Note that $\operatorname{Pic}\left(\mathfrak{X}_{+}\right)$ is generated by $\varphi^{*} \operatorname{Pic}\left(\mathfrak{X}_{-}\right)$and $L^{ \pm}$; therefore any element in $K\left(\mathfrak{X}_{+}\right)$can be written as a Laurent polynomial of $L$ with coefficients in $K\left(\mathfrak{X}_{-}\right)$. Using the above relation (7.27), we find that every element of $K\left(\mathfrak{X}_{+}\right)$can be written in the form

$$
\sum_{k=-h}^{J-h} a_{k} \cdot L^{k} \quad \text { with } a_{k} \in K\left(\mathfrak{X}_{-}\right),
$$

which can also be rewritten as a $K\left(\mathfrak{X}_{-}\right)$-linear combination of 1 and $(1-L) L^{k}=\left[\mathcal{O}_{E}(-k E)\right]$ with $-h \leq k \leq J-h-1$. Part (b) follows. The theorem is proved.

\subsubsection{Orlov's decomposition as a sectorial decomposition of the quantum D-module}

Next we see that this Orlov-type decomposition actually arises from a sectorial decomposition of the quantum D-module of $\mathfrak{X}_{+}-$as appears in Proposition 6.5 - associated with some $\tau_{+} \in$ $H_{\mathrm{CR}}^{*}\left(\mathfrak{X}_{+}\right)$; the point $\tau_{+}$can be very far from the large radius limit point and is not explicit in general (see however Example 7.34).

The analytic quantum D-module (6.1) is originally defined in a neighbourhood of the large radius limit point. In the following theorem, we consider analytic continuation of the quantum D-module along certain paths in $H_{\mathrm{CR}}^{*}\left(\mathfrak{X}_{ \pm}\right)$; we choose a real class $\tau_{\star, \pm} \in H_{\mathrm{CR}}^{*}\left(\mathfrak{X}_{ \pm} ; \mathbb{R}\right)$ which is sufficiently close to the large radius limit point as a base point (see Remark 2.8).

Theorem 7.33. There exist paths from $\tau_{\star, \pm}$ to $\tau_{ \pm} \in H_{\mathrm{CR}}^{*}\left(\mathfrak{X}_{ \pm}\right)$and analytic continuation of the quantum D-modules $\mathrm{QDM}^{\mathrm{an}}\left(\mathfrak{X}_{ \pm}\right)$along these paths with the following properties.

(1) The eigenvalues of the Euler multiplication $E_{\star_{\tau_{+}}}$at $\tau_{+}$have mutually distinct imaginary parts, and we have the following sectorial decomposition as in Proposition 6.5

$$
\Phi^{+}:\left.\pi^{*} \operatorname{QDM}^{\mathrm{an}}\left(\mathfrak{X}_{+}\right)\right|_{W \times I} \cong \bigoplus_{i=1}^{N_{+}}\left(\mathcal{A}_{W \times I}, d+d\left(\mathbf{u}_{i} / z\right)\right)
$$

over an open neighbourhood $W$ of $\tau_{+}$in $H_{\mathrm{CR}}^{*}\left(\mathfrak{X}_{+}\right)$, where $\pi: H_{\mathrm{CR}}^{*}\left(\mathfrak{X}_{+}\right) \times \widetilde{\mathbb{C}} \rightarrow H_{\mathrm{CR}}^{*}\left(\mathfrak{X}_{+}\right) \times \mathbb{C}$ is the oriented real blow-up, $I=\left\{\left(z, e^{\mathbf{i} \theta}\right) \in \widetilde{\mathbb{C}}:|\theta|<\frac{\pi}{2}+\epsilon\right\}$ (for some $\epsilon>0$ ) and $\mathbf{u}_{1}, \ldots, \mathbf{u}_{N_{+}}$are the eigenvalues of the Euler multiplication. 
(2) Moreover, there exists a holomorphic submersion $f: W \rightarrow H_{\mathrm{CR}}^{*}\left(\mathfrak{X}_{-}\right)$with $f\left(\tau_{+}\right)=\tau_{-}$such that we have the similar sectorial decomposition for $\mathfrak{X}_{-}$

$$
\Phi^{-}:\left.\pi^{*} f^{*} \operatorname{QDM}^{\mathrm{an}}\left(\mathfrak{X}_{-}\right)\right|_{W \times I} \cong \bigoplus_{i=1}^{N_{-}}\left(\mathcal{A}_{W \times I}, d+d\left(\mathbf{u}_{i} / z\right)\right),
$$

where $W$ and $I$ are the same as Part (1) and $\mathbf{u}_{1}, \ldots, \mathbf{u}_{N_{-}}$are the pull-backs along $f$ of the eigenvalues of the Euler multiplication in the quantum cohomology of $\mathfrak{X}_{-}$which form a subset of $\left\{\mathbf{u}_{1}, \ldots, \mathbf{u}_{N_{+}}\right\}$in Part (1).

(3) The eigenvalues $\left\{\mathbf{u}_{1}, \ldots, \mathbf{u}_{N_{+}}\right\}$in Part (1) are divided into $J+1$ groups $\bigsqcup_{k=-h}^{J-h-1}\left\{\mathbf{u}_{i}^{(k)}: 1 \leq\right.$ $i \leq r\} \sqcup\left\{\mathbf{u}_{1}, \ldots, \mathbf{u}_{N_{-}}\right\}$satisfying

$$
\Im\left(\mathbf{u}_{i_{1}}^{(-h)}\right)>\cdots>\Im\left(\mathbf{u}_{i_{h}}^{(-1)}\right)>\Im\left(\mathbf{u}_{j}\right)>\Im\left(\mathbf{u}_{i_{h+1}}^{(0)}\right)>\cdots>\Im\left(\mathbf{u}_{i_{J}}^{(J-h-1)}\right)
$$

for all $i_{k} \in\{1, \ldots, r\}$ (with $\left.1 \leq k \leq J\right)$ and $j \in\left\{1, \ldots, N_{-}\right\}$.

(4) There exist $K$-classes $V_{i}^{ \pm} \in K\left(\mathfrak{X}_{ \pm}\right), 1 \leq i \leq N_{ \pm}$such that $\Phi^{ \pm}\left(e^{\mathbf{u}_{i} / z} s_{i}^{ \pm}\right)=e_{i}$, where $s_{i}^{ \pm}$is the flat section associated with $V_{i}^{ \pm}$via the $\widehat{\Gamma}$-integral structure (Definition 2.7 ) (which is analytically continued from $\tau_{\star, \pm}$ through the specified paths) and $e_{i}$ denotes the ith standard basis. Moreover, we have

(a) $V_{i}^{-}, 1 \leq i \leq N_{-}$form a basis of $K\left(\mathfrak{X}_{-}\right)$;

(b) $\left\{V_{i}^{+}: \mathbf{u}_{i}=\mathbf{u}_{j}^{(k)}\right.$ for some $\left.j\right\}$ gives a basis of $K(Z)_{k} \subset K\left(\mathfrak{X}_{+}\right)$;

(c) $V_{i}^{+}=\varphi^{*} V_{i}^{-}$for $1 \leq i \leq N_{-}$.

In particular, we have a sectorial decomposition

$$
\left.\left.\pi^{*} \operatorname{QDM}^{\mathrm{an}}\left(\mathfrak{X}_{+}\right)\right|_{W \times I} \cong \mathscr{R}_{-h}^{\mathrm{an}} \oplus \cdots \oplus \mathscr{R}_{-1}^{\mathrm{an}} \oplus \pi^{*} f^{*} \mathrm{QDM}^{\mathrm{an}}\left(\mathfrak{X}_{-}\right)\right|_{W \times I} \oplus \mathscr{R}_{0}^{\mathrm{an}} \oplus \cdots \oplus \mathscr{R}_{J-h-1}^{\mathrm{an}},
$$

which corresponds to the decomposition (7.23) of the $K$-group via the $\widehat{\Gamma}$-integral structure, where $\mathscr{R}_{k}^{\mathrm{an}}=\bigoplus_{i=1}^{r_{k}}\left(\mathcal{A}_{W \times I}, d+d\left(\mathbf{u}_{i}^{(k)} / z\right)\right)$.

Proof. Throughout Section 7, we have assumed that $S_{-}=S \cap \Delta_{-}$and $S=S_{-} \cup\{\hat{b}\}$ so that $\mathcal{M}$ is the small quantum cohomology locus of $\mathfrak{X}_{+}$. The construction of the sectorial decompositions (7.14), however, does not require this assumption because $S$ can be arbitrarily large in the discussion of Sections 5-6. We choose a large enough finite set $\widehat{S} \subset \mathbf{N} \cap \Pi$ containing $S$ such that the corresponding mirror map mir $_{+}$is generically submersive (this is possible by the argument in [27, Section 7.4]). Let $\widehat{\mathcal{M}} \supset \mathcal{M}$ denote the base of the LG model corresponding to $\widehat{S}$. The discussion at the beginning of Section 7.5 yields, for any $q_{0} \in \mathcal{V}_{0}^{\text {ss }}$ and an admissible phase $\phi$ for the critical values of $F_{q_{0}}$, the following decompositions (similarly to (7.14))

$$
\left.\pi^{*} \operatorname{mir}_{ \pm}^{*} \operatorname{QDM}^{\mathrm{an}}\left(\mathfrak{X}_{ \pm}\right)\right|_{\widehat{B} \times I_{\phi}} \cong \bigoplus_{i=1}^{N_{ \pm}}\left(\mathcal{A}_{\widehat{B} \times I_{\phi}}, d+d\left(\mathbf{u}_{i} / z\right)\right)
$$

over a neighbourhood $\widehat{B}$ of $q_{0}$ in $\widehat{\mathcal{M}}$. Here $\left(\mathbf{u}_{1}, \ldots, \mathbf{u}_{N_{+}}\right)$are the eigenvalues of $E \star_{\tau}$ in the quantum cohomology of $\mathfrak{X}_{+}$at $\tau=\operatorname{mir}_{+}(q)$, and first $N_{-}\left(\mathbf{u}_{1}, \ldots, \mathbf{u}_{N_{-}}\right)$of them are the eigenvalues of $E \star_{\tau}$ in the quantum cohomology of $\mathfrak{X}_{-}$at $\tau=\operatorname{mir}_{-}(q)$, where $q$ varies in $\widehat{B}$. By the choice of $\widehat{S}$, the eigenvalues $\mathbf{u}_{1}, \ldots, \mathbf{u}_{N_{+}}$are pairwise distinct at generic points in $\widehat{B}$ (see Remark 6.2). 
Suppose now that $q_{0}$ is a point from $\bigcup_{0<t<t_{1}} \mathbf{s}_{t}\left(\mathcal{V}_{-, \mathbb{R}}^{\mathrm{sm}, \mathrm{ss}} \cap \mathbb{D}_{\mathrm{sm}}^{\times \circ}\right) \cap \mathcal{V}_{0}^{\mathrm{ss}}$ and $|\phi|$ is sufficiently small so that the conclusion of Theorem 7.25 holds; then there exist $K$-classes $V_{i}^{\circ \pm} \in K\left(\mathfrak{X}_{ \pm}\right)$such that the associated flat sections $s_{i}^{ \pm}$(via the $\widehat{\Gamma}$-integral structure) correspond to $e^{-\mathbf{u}_{i} / z} e_{i}$ under the decomposition (7.28) and that $V_{i}^{\circ+}=\varphi^{*} V_{i}^{\circ-}$ for $1 \leq i \leq N_{-}$. Choose a point $q^{\circ} \in \widehat{B}$ such that the corresponding eigenvalues $\mathbf{u}_{1}^{\circ}, \ldots, \mathbf{u}_{N_{+}}^{\circ}$ are pairwise distinct, and set $\tau_{ \pm}^{\circ}=\operatorname{mir}_{ \pm}\left(q^{\circ}\right)$; we regard $\tau_{ \pm}^{\circ}$ as elements of $H_{\mathrm{CR}}^{*}\left(\mathfrak{X}_{ \pm}\right)$(rather than their images in $\left[\mathcal{M}_{\mathrm{A}}\left(\mathfrak{X}_{ \pm}\right) / \mathrm{Pic}^{\mathrm{st}}\left(\mathfrak{X}_{ \pm}\right)\right]$). Let $C_{N}$ denote the configuration space of distinct $N$ points in $\mathbb{C}$ :

$$
C_{N}:=\left\{\left(\mathbf{u}_{1}, \ldots, \mathbf{u}_{N}\right) \in \mathbb{C}^{N}: \mathbf{u}_{i} \neq \mathbf{u}_{j} \text { if } i \neq j\right\} / \mathfrak{S}_{N}
$$

Since the eigenvalues of $E \star_{\tau}$ form a local co-ordinate system on $H^{*}\left(\mathfrak{X}_{ \pm}\right)$near a semisimple point, we can identify a neighbourhood of $\left(\mathbf{u}_{1}^{\circ}, \ldots, \mathbf{u}_{N_{+}}^{\circ}\right)$ in $C_{N_{ \pm}}$with a neighbourhood of $\tau_{ \pm}^{\circ}$ in $H_{\mathrm{CR}}^{*}\left(\mathfrak{X}_{ \pm}\right)$. Let $\widetilde{C}_{N}$ denote the universal cover of $C_{N}$. By isomonodromic deformation [40, Theorem 4.7], there exist analytic hypersurfaces ${ }^{23} H_{ \pm} \subset \widetilde{C}_{N_{ \pm}}$such that the quantum connection in a neighbourhood of $\tau_{ \pm}^{\circ} \in H_{\mathrm{CR}}^{*}\left(\mathfrak{X}_{ \pm}\right)$can be extended to a meromorphic flat connection $\nabla$ on the trivial bundle $H_{\mathrm{CR}}^{*}\left(\mathfrak{X}_{ \pm}\right) \times\left(\widetilde{C}_{N_{ \pm}}^{\circ} \times \mathbb{C}\right) \rightarrow \widetilde{C}_{N_{ \pm}}^{\circ} \times \mathbb{C}$ of the form

$$
\nabla=d+\frac{1}{z} A+\left(-\frac{1}{z^{2}} U+\frac{1}{z} V\right) d z
$$

where $\widetilde{C}_{N_{ \pm}}^{\circ}:=\widetilde{C}_{N_{ \pm}} \backslash H_{ \pm}, A$ is an $\operatorname{End}\left(H_{\mathrm{CR}}^{*}\left(\mathfrak{X}_{ \pm}\right)\right)$-valued 1 -form on $\widetilde{C}_{N_{ \pm}}^{\circ}, U$ and $V$ are $\operatorname{End}\left(H_{\mathrm{CR}}^{*}\left(\mathfrak{X}_{ \pm}\right)\right)$-valued functions on $\widetilde{C}_{N_{ \pm}}^{\circ}$ and $A, U, V$ are independent of $z$. Here the eigenvalues of $U$ give the co-ordinates $\left(\mathbf{u}_{1}, \ldots, \mathbf{u}_{N_{ \pm}}\right)$on $\widetilde{C}_{N_{ \pm}}$. Moreover, by taking bigger hypersurfaces $H_{ \pm}$ if necessary, we may assume that this defines a Frobenius manifold structure on $\widetilde{C}_{N_{ \pm}}^{\circ}$. By choosing a basis $\left\{\phi_{i}\right\}$ of $H_{\mathrm{CR}}^{*}\left(\mathfrak{X}_{ \pm}\right)$, we can determine the flat vector fields $\frac{\partial}{\partial \tau^{i}}$ by $A_{\partial / \partial \tau^{i}} 1=\phi_{i}$. In particular, we have a flat co-ordinate system $\left(\widetilde{C}_{N_{ \pm}}^{\circ}\right)^{\sim} \rightarrow H_{\mathrm{CR}}^{*}\left(\mathfrak{X}_{ \pm}\right)$on the universal cover $\left(\widetilde{C}_{N_{ \pm}}^{\circ}\right)^{\sim}$ which equals $\tau_{ \pm}^{\circ}$ at $\left(\mathbf{u}_{1}^{\circ}, \ldots, \mathbf{u}_{N_{ \pm}}^{\circ}\right)$. We also have a submersion $g: \widetilde{C}_{N_{+}} \rightarrow \widetilde{C}_{N_{-}}$sending $\left(\mathbf{u}_{1}, \ldots, \mathbf{u}_{N_{+}}\right)$to $\left(\mathbf{u}_{1}, \ldots, \mathbf{u}_{N_{-}}\right)$; this induces a submersion $g: \widetilde{C}_{N_{+}}^{\circ \circ} \rightarrow \widetilde{C}_{N_{-}}^{\circ}$, where $\widetilde{C}_{N_{+}}^{\circ \circ}:=$ $\widetilde{C}_{N_{+}} \backslash\left(H_{+} \cup g^{-1}\left(H_{-}\right)\right) \subset \widetilde{C}_{N_{+}}^{\circ}$.

We may assume that $q^{\circ}$ is sufficiently close to $q_{0}$ so that $\left\{\mathbf{u}_{1}^{\circ}, \ldots, \mathbf{u}_{N_{+}}^{\circ}\right\}$ are contained in $B_{\rho_{2}}(0) \sqcup \bigsqcup_{k \in \mathbb{Z} / J \mathbb{Z}} \mathfrak{B}_{k}(t)$, where $0<t<t_{1}$ is the number such that $q_{0} \in \operatorname{Im}\left(\mathbf{s}_{t}\right)$. Moving $\mathbf{u}_{i}^{\circ}$ along the strip regions $\mathcal{S}_{\text {conv }} \cup \mathcal{S}_{-h} \cup \cdots \cup \mathcal{S}_{J-h-1}$, we can connect $\left(\mathbf{u}_{1}^{\circ}, \ldots, \mathbf{u}_{N_{+}}^{\circ}\right)$ by a continuous path $\gamma$ inside $\widetilde{C}_{N_{+}}^{\circ}$ with a point $\left(\mathbf{u}_{1}^{\diamond}, \ldots, \mathbf{u}_{N_{+}}^{\diamond}\right) \in \widetilde{C}_{N_{+}}^{\circ \circ}$ having mutually distinct imaginary parts: see Fig. 15. The numbers $\mathbf{u}_{1}^{\diamond}, \ldots, \mathbf{u}_{N_{+}}^{\diamond}$ are divided into $J+1$ groups as in Part (3) of the statement, depending on which strip regions they belong to. Let $\tau_{+} \in H_{\mathrm{CR}}^{*}\left(\mathfrak{X}_{+}\right)$be the analytically continued (along the path $\gamma$ ) flat co-ordinate at the point $\left(\mathbf{u}_{1}^{\diamond}, \ldots, \mathbf{u}_{N_{+}}^{\diamond}\right) \in \widetilde{C}_{N_{+}}^{\circ}$ and $\tau_{-} \in H_{\mathrm{CR}}^{*}\left(\mathfrak{X}_{-}\right)$be the analytically continued (along the path $g(\gamma)$ ) flat co-ordinate at the point $g\left(\mathbf{u}_{1}^{\diamond}, \ldots, \mathbf{u}_{N_{+}}^{\diamond}\right)=\left(\mathbf{u}_{1}^{\diamond}, \ldots, \mathbf{u}_{N_{-}}^{\diamond}\right) \in \widetilde{C}_{N_{-}}^{\circ}$. By the above construction, the quantum D-modules of $\mathfrak{X}_{ \pm}$are analytically continued to $\tau_{ \pm}$along certain paths, and the submersion $g$ induces, when written in flat co-ordinates, a submersion $f$ from a neighbourhood of $\tau_{+}$in $H_{\mathrm{CR}}^{*}\left(\mathfrak{X}_{+}\right)$to a neighbourhood of $\tau_{-}$in $H_{\mathrm{CR}}^{*}\left(\mathfrak{X}_{-}\right)$with $f\left(\tau_{+}\right)=\tau_{-}$.

We claim that the statement of the theorem holds for $\tau_{ \pm}, f$ and a sufficiently small neighbourhood $W$ of $\tau_{+}$. Parts (1), (2) follow from the construction and the Hukuhara-Turrittin decomposition (Proposition 6.5 ); note that 0 is an admissible phase for $\left(\mathbf{u}_{1}^{\diamond}, \ldots, \mathbf{u}_{N_{ \pm}}^{\diamond}\right)$. Part (3)

\footnotetext{
${ }^{23}$ In the original version of the present paper, we did not delete the hypersurfaces $H_{ \pm}$from $\widetilde{C}_{N_{ \pm}}$. The need for the deletion was pointed out by one of the referees.
} 


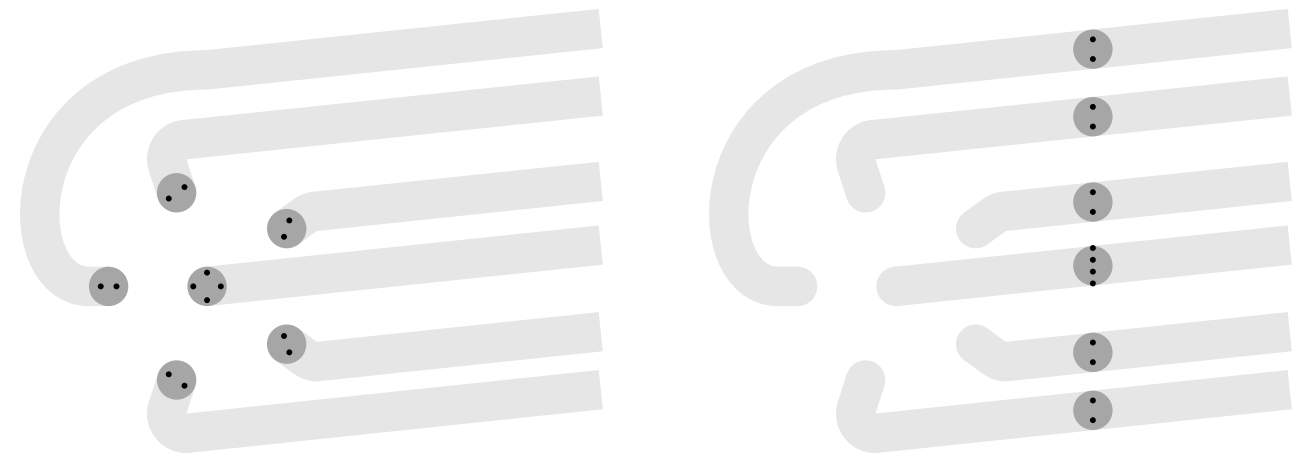

Figure 15. A path from $\left(\mathbf{u}_{1}^{\circ}, \ldots, \mathbf{u}_{N_{+}}^{\circ}\right)$ to $\left(\mathbf{u}_{1}^{\diamond}, \ldots, \mathbf{u}_{N_{+}}^{\diamond}\right)$ : we move the left configuration $\left(\mathbf{u}_{i}^{\circ}\right)$ to the right one $\left(\mathbf{u}_{i}^{\diamond}\right)$ along the strip regions.

has been already achieved. We show Part (4). Recall that the decomposition (7.28) corresponds to the basis $\left\{V_{i}^{\text {o士 }}\right\}$ of $K\left(\mathfrak{X}_{ \pm}\right)$under the $\widehat{\Gamma}$-integral structure and it gives rise to the asymptotic basis (see Section 6.2) associated with $\tau_{ \pm}^{\circ}$ and $\phi$. As we move $\left(\mathbf{u}_{1}, \ldots, \mathbf{u}_{N_{ \pm}}\right)$along the path $\gamma$ (or $g(\gamma)$ ) and change the phase from $\phi$ to zero, this asymptotic basis undergoes mutation as explained in Section 6.2. The basis $\left\{V_{i}^{ \pm}\right\} \subset K\left(\mathfrak{X}_{ \pm}\right)$in Part (4) arises from $\left\{V_{i}^{\circ \pm}\right\}$ by this mutation. Part (4-a) is obvious from this construction. By the same argument as in the last paragraph of the proof of Theorem 7.25, we see that the relation $V_{i}^{\circ+}=\varphi^{*} V_{i}^{\circ-}$ is preserved by mutation, and we get $V_{i}^{+}=\varphi^{*} V_{i}^{-}$for $1 \leq i \leq N_{-}$as required in Part (4-c). Let $\Gamma_{i}^{\pi+\phi}$ denote the Lefschetz thimbles (6.12) of $F_{q_{0}}$ corresponding to the $i$ th component of the decomposition (7.28) (recall that components in (7.28) are naturally indexed by critical points of $F_{q_{0}}$; see Proposition 6.18). As discussed in the proof of Proposition 7.8, $V_{i}^{\circ+}$ equals the $K$-class $V\left(\Gamma_{i}^{\pi+\phi}\right)$ associated with $\Gamma_{i}^{\pi+\phi}$ under the correspondence in Theorem 7.5. Note by Remark 7.6 that

$$
\chi\left(V_{i}^{\circ+}, V_{j}^{\circ+}\right)=(-1)^{n(n-1) / 2} \#\left(e^{-\pi \mathbf{i}} \Gamma_{i}^{\pi+\phi} \cdot \Gamma_{j}^{\pi+\phi}\right) .
$$

Therefore, mutation of $\left\{V_{i}^{\circ+}\right\}$ corresponds to Picard-Lefschetz transformation (see [7, Chapter I]) of thimbles $\left\{\Gamma_{i}^{\pi+\phi}\right\}$; here we consider Picard-Lefschetz transformation with $q_{0} \in \mathcal{M}$ fixed. We transform $\left\{\Gamma_{i}^{\pi+\phi}\right\}$ by the sequence of Picard-Lefschetz transformations corresponding to the sequence of mutations that $\left\{V_{i}^{\circ+}\right\}$ undergoes and obtain a new basis $\left\{\Gamma_{i}\right\}$ of $\operatorname{Lef}_{q_{0}}$. The basis $\left\{\Gamma_{i}\right\}$ is the union of bases of $\operatorname{Lef}_{q_{0}}^{(k)}$ and $\operatorname{Lef}_{q_{0}}^{\text {conv }} ;\left\{\Gamma_{i}: \mathbf{u}_{i}=\mathbf{u}_{j}^{(k)}\right.$ for some $\left.j\right\}$ gives a basis of Lef $q_{0}^{(k)}$. Since we have $V_{i}^{+}=V\left(\Gamma_{i}\right)$, Part (4-b) follows from Theorem 7.31. The theorem is proved.

Example 7.34. We give an example where the Orlov-type decomposition occurs at an explicit $\tau_{+}$. Set $X_{-}:=\mathbb{P}^{4}$ and let $X_{+}$be the blowup of $X_{-}$along a line $Z=\mathbb{P}^{1} \subset X_{-}$. Both $X_{+}$and $X_{-}$are Fano, and their small quantum cohomologies are defined over polynomial rings. Planes in $X_{-}=\mathbb{P}^{4}$ containing the line $Z$ are parametrized by $\mathbb{P}^{2}$, and hence we have a natural projection $X_{+} \rightarrow \mathbb{P}^{2}$. Thus $X_{+}$is a $\mathbb{P}^{2}$-bundle over $\mathbb{P}^{2}: X_{+} \cong \mathbb{P}_{\mathbb{P}^{2}}(\mathcal{O} \oplus \mathcal{O} \oplus \mathcal{O}(-1))$. Let $p_{1}$ be the pull-back of the ample class in $H^{2}\left(\mathbb{P}^{2} ; \mathbb{Z}\right)$ and let $p_{2}=\varphi^{*}(p)$ be the pull-back of the ample class $p$ in $H^{2}\left(X_{-} ; \mathbb{Z}\right)$. They form a nef basis of $H^{2}\left(X_{+} ; \mathbb{Z}\right)$. The class of the exceptional divisor is given by $[E]=p_{1}-p_{2}$. The uncompactified LG mirror of $X_{+}$is given by the family of Laurent polynomials

$$
F_{q_{1}, q_{2}}=x_{1}+x_{2}+x_{3}+x_{4}+\frac{q_{1} q_{2}}{x_{1} x_{2} x_{3} x_{4}}+\frac{1}{q_{1}} x_{1} x_{2} x_{3}
$$


parametrized by $\left(q_{1}, q_{2}\right) \in\left(\mathbb{C}^{\times}\right)^{2}$. The large radius limit (LRL) point for $X_{+}$corresponds to $q_{1}=q_{2}=0$. The mirror map for $X_{+}$is trivial: $\operatorname{mir}_{+}\left(q_{1}, q_{2}\right)=p_{1} \log q_{1}+p_{2} \log q_{2}$. On the other hand, the uncompactified LG mirror of $X_{-}$is given by the family

$$
F_{q, t}=x_{1}+x_{2}+x_{3}+x_{4}+\frac{q}{x_{1} x_{2} x_{3} x_{4}}+t x_{1} x_{2} x_{3},
$$

where the LRL point for $X_{-}$is $q=t=0$. The two families are related by the change of coordinates $q=q_{1} q_{2}, t=q_{1}^{-1}$. The small quantum cohomology locus for $X_{-}$is $t=0$. It is not easy to find a closed formula for the mirror map of $X_{-}$, but we know that it has the asymptotic form $\operatorname{mir}_{-}(q, t) \sim p \log q+t \cdot p^{3}$ as $(q, t) \rightarrow(0,0)$ (see Remark 4.10) and that $\operatorname{mir}_{-}(q, 0)=p \log q$. The Euler vector field gives a grading $\operatorname{deg} q_{1}=2, \operatorname{deg} q_{2}=3$ and $\operatorname{deg} t=-2, \operatorname{deg} q=5$ (in complex unit). Define a dimensionless parameter $\lambda:=q_{1}^{-\frac{3}{2}} q_{2}=t^{\frac{5}{2}} q$. Critical points/values of $F_{q_{1}, q_{2}}=F_{q, t}$ are given by

$$
x_{1}=x_{2}=x_{3}=t^{-\frac{1}{2}} x, \quad x_{4}=t^{-\frac{1}{2}}\left(x+x^{3}\right), \quad F_{q, t}(\mathrm{cr})=t^{-\frac{1}{2}}\left(5 x+3 x^{3}\right),
$$

where $x$ is a root of $x^{5}\left(x^{2}+1\right)^{2}=\lambda$. The discriminant locus (where $x$ has multiple roots) is $\lambda=0$ or $\pm 400 \sqrt{5} \mathbf{i} / 3^{9}$. Therefore the quantum cohomology is semisimple over the positive real locus $q_{1}>0, q_{2}>0$. The critical values have the asymptotics

$$
F_{q, t}(\mathrm{cr})=\left\{\begin{array}{l}
t^{-\frac{1}{2}}\left(5 \lambda^{\frac{1}{5}}+\lambda^{\frac{3}{5}}+O(\lambda)\right), \\
t^{-\frac{1}{2}}\left(2 \mathbf{i} \pm 2 \sqrt{\mathbf{i}} \lambda^{\frac{1}{2}}+O(\lambda)\right), \\
t^{-\frac{1}{2}}\left(-2 \mathbf{i} \pm 2 \sqrt{-\mathbf{i}} \lambda^{\frac{1}{2}}+O(\lambda)\right)
\end{array} \quad=\left\{\begin{array}{l}
5 q^{\frac{1}{5}}+t q^{\frac{3}{5}}+O\left(t^{2}\right), \\
2 \mathbf{i} t^{-\frac{1}{2}} \pm 2 \sqrt{\mathbf{i}} q^{\frac{1}{2}} t^{\frac{3}{4}}+O\left(t^{2}\right), \\
-2 \mathbf{i} t^{-\frac{1}{2}} \pm 2 \sqrt{-\mathbf{i}} q^{\frac{1}{2}} t^{\frac{3}{4}}+O\left(t^{2}\right) .
\end{array}\right.\right.
$$

as $\lambda \rightarrow 0$ (or $t \rightarrow 0$ with $q$ fixed) and

$$
F_{q, t}(\mathrm{cr})=t^{-\frac{1}{2}}\left(3 \lambda^{\frac{1}{3}}+3 \lambda^{\frac{1}{9}}+O\left(\lambda^{-\frac{1}{9}}\right)\right)=3 q_{2}^{\frac{1}{3}}+3 q_{1}^{\frac{1}{3}} q_{2}^{\frac{1}{9}}+O\left(q_{1}^{\frac{2}{3}}\right)
$$

as $\lambda \rightarrow \infty$ (or $q_{1} \rightarrow 0$ with $q_{2}$ fixed). The first line of (7.29) gives 5 'convergent' critical values (depending on the choice of $q^{\frac{1}{5}}$ ) and the next two lines give 4 'divergent' critical values around $t=0$. They have mutually distinct imaginary parts when $t>0$ is sufficiently close to zero and $q>0$. We will identify the corresponding elements in the $K$-group for the phase $\phi=0$.

We consider a path where the parameter $\lambda \in \mathbb{R}$ increases from 0 to $\infty$. It is convenient to take $t=t(\lambda)=\lambda^{\frac{2}{3}}+\lambda^{\frac{2}{5}}$ so that $\left(q_{1}, q_{2}\right) \sim\left(\lambda^{-\frac{3}{2}}, 1\right)$ as $\lambda \rightarrow \infty$ and $(q, t) \sim\left(1, \lambda^{\frac{2}{5}}\right)$ as $\lambda \rightarrow 0$. The move of the critical values is shown in Fig. 16. When $\lambda$ is large, say $\lambda=12.5$, we can determine the mirror partners (in the $K$-group) of some Lefschetz thimbles by using the monodromy action on the $\left(q_{1}, q_{2}\right)$-space: see the rightmost picture of Fig. 16 . Here $H_{1}, H_{2}$ denote the pull-backs of the hyperplanes on $\mathbb{P}^{2}$ and $\mathbb{P}^{4}$ respectively (so that $p_{i}$ is the Poincaré dual of $H_{i}$ ). A numerical calculation on computer shows that, as $\lambda$ decreases from $\lambda=12.5$ to $\lambda=0.0009$, the thimble corresponding to $\mathcal{O}\left(-H_{1}\right)$ undergoes a Picard-Lefschetz transformation with respect to that corresponding to $\mathcal{O}\left(-H_{2}\right)$. As we saw in the proof of Theorem 7.33 , this corresponds to mutation in $K$-group. After (left) mutation, $\mathcal{O}\left(-H_{1}\right)$ becomes

$$
\mathcal{E}=\text { Cone }\left(\operatorname{Hom}^{\bullet}\left(\mathcal{O}\left(-H_{2}\right), \mathcal{O}\left(-H_{1}\right)\right) \otimes \mathcal{O}\left(-H_{2}\right) \rightarrow \mathcal{O}\left(-H_{1}\right)\right)=\mathcal{O}_{E}\left(E-H_{2}\right) .
$$

Considering the monodromy action near $t=0$ and performing further mutation, we find that the exceptional collection (adapted to the decomposition (7.23) with $J=2, h=1$ )

$$
\begin{aligned}
& \mathcal{E}=\mathcal{O}_{E}\left(E-H_{2}\right), \quad \mathcal{O}_{E}(E), \quad \mathcal{O}\left(-H_{2}\right), \quad \mathcal{G}=\mathcal{O}\left(-2 H_{2}\right) \otimes \varphi^{*} T_{\mathbb{P}^{4}}[-1], \quad \mathcal{O}, \\
& \mathcal{H}=\mathcal{O}\left(2 H_{2}\right) \otimes \varphi^{*} \Omega_{\mathbb{P}^{4}}^{1}, \quad \mathcal{O}\left(H_{2}\right), \quad \mathcal{O}_{E}[-1], \quad \mathcal{F}=\mathcal{O}_{E}\left(H_{2}\right)[-1]
\end{aligned}
$$



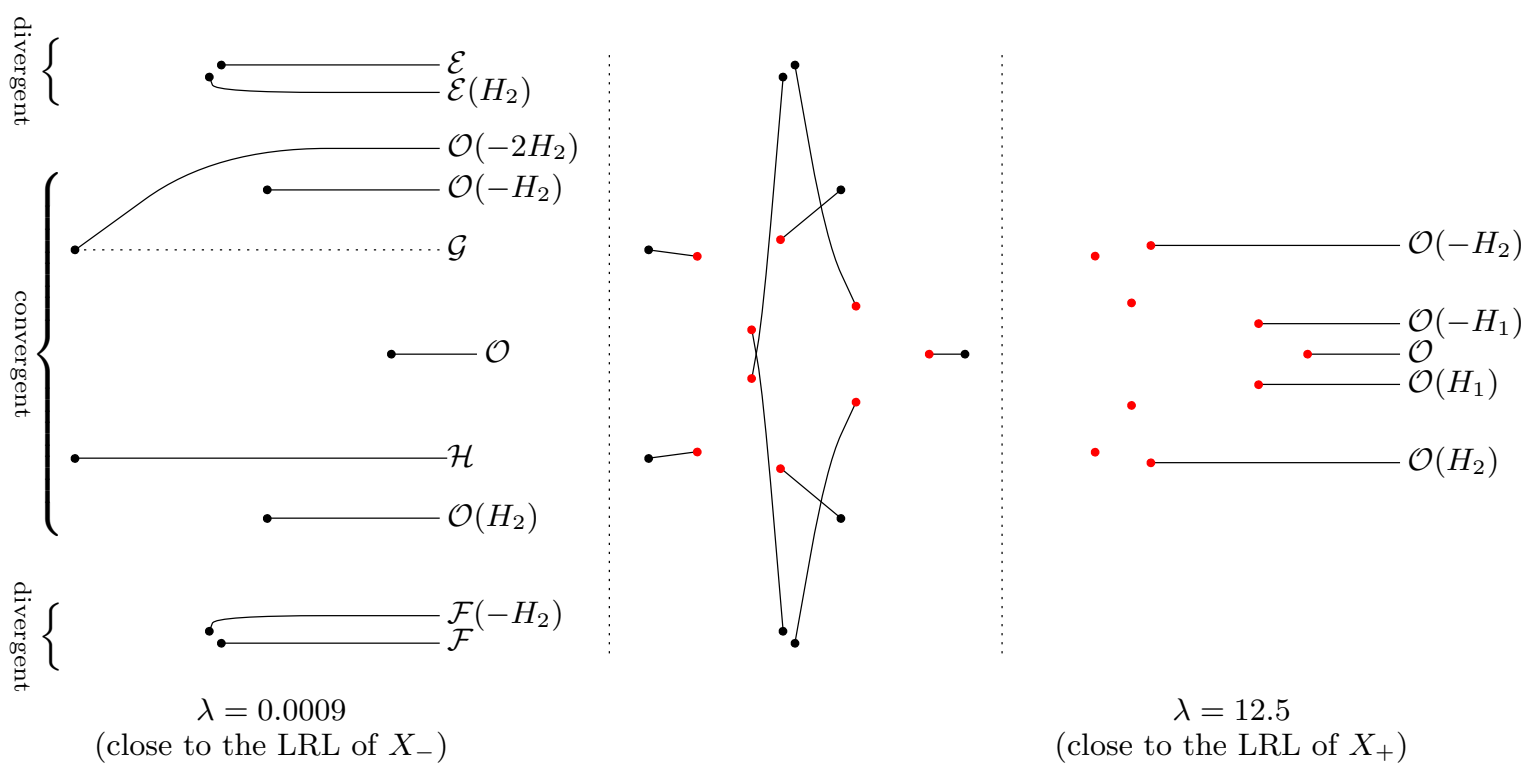

$\lambda=12.5$
(close to the LRL of $X_{+}$)

Figure 16. Move of critical values: the trajectories of the critical values are shown in the middle picture; $\mathcal{E}$ is the left mutation of $\mathcal{O}\left(-H_{1}\right)$ with respect to $\mathcal{O}\left(-H_{2}\right)$; similarly $\mathcal{F}$ is the right mutation of $\mathcal{O}\left(H_{1}\right)$ with respect to $\mathcal{O}\left(H_{2}\right) ; \mathcal{G}$ is the right mutation of $\mathcal{O}\left(-2 H_{2}\right)$ with respect to $\mathcal{O}\left(-H_{2}\right)$.

corresponds to the Lefschetz thimbles $\Gamma_{1}^{\pi}, \ldots, \Gamma_{9}^{\pi}(6.12)$ ordered in such a way that the imaginary parts of the corresponding critical values decrease $\Im\left(F\left(\mathrm{cr}_{1}\right)\right)>\Im\left(F\left(\mathrm{cr}_{2}\right)\right)>\cdots>\Im\left(F\left(\mathrm{cr}_{9}\right)\right)$. These are the classes $V_{i}^{+}$in Theorem 7.33(4) in this case. Note that the corresponding sectorial decomposition occurs at a point $\tau_{+}=[E] \log t+p_{2} \log q \in H^{2}\left(X_{+}\right), q, t>0$ such that $t$ is sufficiently small (in the leftmost picture of Fig. $16, t \approx 0.0698, q \approx 0.698$ ).

Remark 7.35. We can use Theorem 7.33 to prove Gamma conjecture II [44, Section 4.6] in some cases. By applying Theorem 7.33 to the case where $Z$ is a (non-stacky) point, we know that if the Gamma conjecture II holds for a weak-Fano compact toric stack $\mathfrak{X}_{-}$, then it also holds for a weighted blowup $\mathfrak{X}_{+}$of $\mathfrak{X}_{-}$at a non-stacky torus-fixed point (as long as $\mathfrak{X}_{+}$is weak-Fano).

\section{Conjecture and discussion}

\subsection{General conjecture}

In view of our main results (Theorems 5.16, 5.19, 7.25, 7.31, and 7.33), we conjecture the following phenomena for more general discrepant birational transformations. Suppose that we have a birational transformation $\varphi: \mathfrak{X}_{+} \rightarrow \mathfrak{X}_{-}$between smooth (not necessarily toric) DM stacks which fits into the diagram

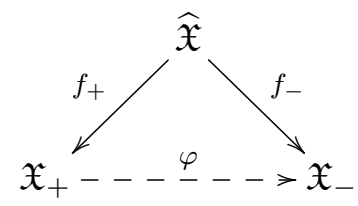

with $\widehat{\mathfrak{X}}$ smooth and $f_{ \pm}$projective birational morphisms, such that $f_{+}^{*} K_{\mathfrak{X}_{+}}-f_{-}^{*} K_{\mathfrak{X}_{-}}$is an effective divisor. We assume that the coarse moduli spaces of $\mathfrak{X}_{ \pm}$are projective, but some of the discussions below can be also adapted to non-compact cases.

We choose a base point $\tau_{\star, \pm} \in H_{\mathrm{CR}}^{*}\left(\mathfrak{X}_{ \pm} ; \mathbb{R}\right)$ which is real and sufficiently close to the large radius limit point. 
Conjecture 8.1 (formal decomposition). There exist paths from $\tau_{\star, \pm}$ to $\tau_{ \pm} \in H_{\mathrm{CR}}^{*}\left(\mathfrak{X}_{ \pm}\right)$and a holomorphic map $f$ from a neighbourhood $W$ of $\tau_{+}$in $H_{\mathrm{CR}}^{*}\left(\mathfrak{X}_{+}\right)$to $H_{\mathrm{CR}}^{*}\left(\mathfrak{X}_{-}\right)$with $f\left(\tau_{+}\right)=\tau_{-}$ such that the quantum D-modules $\mathrm{QDM}^{\mathrm{an}}\left(\mathfrak{X}_{ \pm}\right)$are analytically continued along these paths, and that we have the decomposition of the quantum D-modules completed in z (see (6.2)):

$$
\left.\left.\overline{\mathrm{QDM}^{\mathrm{an}}}\left(\mathfrak{X}_{+}\right)\right|_{W} \cong f^{*} \overline{\mathrm{QDM}^{\mathrm{an}}}(\mathfrak{X}-)\right|_{W} \oplus \mathscr{R} \text {. }
$$

Here $\mathscr{R}$ is a locally free $\mathcal{O}_{W} \llbracket z \rrbracket$-module equipped with a meromorphic flat connection $\nabla^{\mathscr{R}}$ and a z-sesquilinear pairing $P^{\mathscr{R}}$, and the formal decomposition respects both the connection and the pairing (in particular it is orthogonal with respect to the pairing (2.9)).

Building on the above conjecture, we can also state a conjecture comparing higher genus Gromov-Witten theories. This involves quantization of the formal decomposition (8.1). See Section 5.5 for the notation.

Conjecture 8.2 (comparison in all genera). The ancestor potentials $\mathscr{A}_{ \pm, \tau}$ of $\mathfrak{X}_{ \pm}$can be analytically continued along the paths from $\tau_{\star, \pm}$ to $\tau_{ \pm}$in Conjecture 8.1. There exist a family $\mathscr{A}_{\tau}^{\prime} \in \mathfrak{A} \mathfrak{F o c k}\left(V_{\tau}, \mathbf{D}_{\tau}\right)$ of tame functions such that

$$
T_{\mathbf{s}} \widehat{U}_{\tau} \mathscr{A}_{+, \tau}=\mathscr{A}_{-, f(\tau)} \otimes \mathscr{A}_{\tau}^{\prime}
$$

for $\tau \in W$, where $\mathbf{s}=\left(-z \phi_{0}, \mathbf{D}_{\tau}\right)+U_{\tau}\left(z \phi_{0}\right), V_{\tau} \subset \mathscr{R}_{\tau}$ is a $\mathbb{C}$-vector subspace such that $\mathscr{R}_{\tau}=V_{\tau} \llbracket z \rrbracket$ and that $P^{\mathscr{R}}$ restricts to the $\mathbb{C}$-valued pairing $V_{\tau} \times V_{\tau} \rightarrow \mathbb{C}, \mathbf{D}_{\tau} \in z \mathscr{R}_{\tau}$ and $U_{\tau}: H_{\mathrm{CR}}^{*}\left(\mathfrak{X}_{+}\right) \llbracket z \rrbracket \cong H_{\mathrm{CR}}^{*}\left(\mathfrak{X}_{-}\right) \llbracket z \rrbracket \oplus V_{\tau} \llbracket z \rrbracket$ is the unitary isomorphism obtained from (8.1) by restricting to $\tau$ and flipping the sign of $z$.

In this conjecture we implicitly assume that the action of the operator $T_{\mathbf{S}}$ on $\widehat{U}_{\tau} \mathscr{A}_{+, \tau}$ is welldefined; this holds if $\mathscr{A}_{+, \tau}$ is rational (see Section 5.5.2). Note also that the space $\mathfrak{A F o c k}\left(V_{\tau}, \mathbf{D}_{\tau}\right)$ itself depends only on $\mathscr{R}_{\tau}$ and $\mathbf{D}_{\tau}$ and does not depend on the choice of $V_{\tau}$, but that $\mathscr{A}_{\tau}^{\prime}$ depends on the choice of $V_{\tau}$.

Next we state a conjecture relating the analytic lift of the formal decomposition (8.1) with a semiorthogonal decomposition of the $K$-group. Recall from (2.11) that the action of $-\nabla_{z^{2} \partial_{z}}$ on $\left.\operatorname{QDM}^{\text {an }}\left(\mathfrak{X}_{ \pm}\right)\right|_{z=0}$ equals the Euler multiplication $E_{\star_{\tau}}$. In particular, the formal decomposition (8.1) implies that $E \star_{\tau}$ on $H_{\mathrm{CR}}^{*}\left(\mathfrak{X}_{+}\right)$is conjugate to $E \star_{f(\tau)} \oplus\left(-\nabla_{z^{2} \partial_{z}}^{\mathscr{R}}\right)$ on $\left.H_{\mathrm{CR}}^{*}\left(\mathfrak{X}_{-}\right) \oplus \mathscr{R}\right|_{z=0}$.

Conjecture 8.3 (analytic lift). We can arrange $\tau_{ \pm}$and the paths from $\tau_{\star, \pm}$ to $\tau_{ \pm}$in Conjecture 8.1 so that (in addition to Conjecture 8.1) the following holds.

(1) We have a decomposition $\left(\mathscr{R}, \nabla^{\mathscr{R}}, P^{\mathscr{R}}\right)=\left(\mathscr{R}_{1}, \nabla^{\mathscr{R}_{1}}, P^{\mathscr{R}_{1}}\right) \oplus\left(\mathscr{R}_{2}, \nabla^{\mathscr{R}_{2}}, P^{\mathscr{R}_{2}}\right)$, where $\mathscr{R}_{i}$ is a locally free $\mathcal{O}_{W} \llbracket z \rrbracket$-module equipped with a flat connection $\nabla^{\mathscr{R}_{i}}$ and a z-sesquilinear pairing $P^{\mathscr{R}_{i}}$. There exist a phase $\phi \in \mathbb{R}$ and real numbers $l_{2}<l_{1}$ such that

(a) all eigenvalues $\mathbf{u}$ of $\left(-\nabla_{z^{2} \partial_{z}}^{\mathscr{R}_{1}}\right)$ on $\left.\mathscr{R}_{1}\right|_{z=0}$ satisfy $\Im\left(e^{-\mathbf{i} \phi} \mathbf{u}\right)>l_{1}$,

(b) all eigenvalues $\mathbf{u}$ of $\left(-\nabla_{z^{2} \partial_{z}}^{\mathscr{R}_{2}}\right)$ on $\left.\mathscr{R}_{2}\right|_{z=0}$ satisfy $\Im\left(e^{-\mathbf{i} \phi} \mathbf{u}\right)<l_{2}$ and

(c) all eigenvalues $\mathbf{u}$ of $E \star_{f(\tau)}$ on $H_{\mathrm{CR}}^{*}\left(\mathfrak{X}_{-}\right)$satisfy $l_{2}<\Im\left(e^{-\mathbf{i} \phi} \mathbf{u}\right)<l_{1}$.

(2) Moreover, we have a sector $I_{\phi}=\left\{\left(r, e^{\mathbf{i} \theta}\right) \in \widetilde{\mathbb{C}}:|\theta-\phi|<\frac{\pi}{2}+\epsilon\right\}$ with some $\epsilon>0$ and an analytic decomposition

$$
\left.\left.\pi^{*} \mathrm{QDM}^{\mathrm{an}}\left(\mathfrak{X}_{+}\right)\right|_{W \times I_{\phi}} \cong \mathscr{R}_{1}^{\mathrm{an}} \oplus \pi^{*} f^{*} \mathrm{QDM}^{\mathrm{an}}\left(\mathfrak{X}_{-}\right)\right|_{W \times I_{\phi}} \oplus \mathscr{R}_{2}^{\mathrm{an}},
$$

where $\pi: W \times \widetilde{\mathbb{C}} \rightarrow W \times \mathbb{C}$ is the oriented real blow-up and $\mathscr{R}_{i}^{\text {an }}$ is a locally free $\mathcal{A}_{W \times I_{\phi}}$ module equipped with a flat connection, such that $\mathscr{R}_{i} \cong \mathscr{R}_{i}^{\mathrm{an}} \otimes_{\mathcal{A}_{W \times I_{\phi}}} \mathcal{O}_{W} \llbracket z \rrbracket, i=1,2$ and that the formal decomposition (8.1) is induced by (8.2). 
(3) Via the $\widehat{\Gamma}$-integral structure for $\mathfrak{X}_{+}$and $\mathfrak{X}_{-}$, the decomposition $(8.2)$ is induced by a semiorthogonal decomposition of the topological K-groups:

$$
K\left(\mathfrak{X}_{+}\right) \cong K_{1} \oplus K\left(\mathfrak{X}_{-}\right) \oplus K_{2}
$$

such that the associated inclusion $K\left(\mathfrak{X}_{-}\right) \hookrightarrow K\left(\mathfrak{X}_{+}\right)$respects the Euler pairing.

Remark 8.4. We expect that the semiorthogonal decomposition (8.3) arises naturally from geometry. In our setting, for example, we could hope that there is a semiorthogonal decomposition of the bounded derived category of $\mathfrak{X}_{+}$of the form (see [9, Conjecture 4.3.7]):

$$
D^{b}\left(\mathfrak{X}_{+}\right)=\left\langle\mathscr{C}_{1}, D^{b}\left(\mathfrak{X}_{-}\right), \mathscr{C}_{2}\right\rangle .
$$

When $\mathfrak{X}_{ \pm}$are toric stacks arising from the variation of GIT quotients as in Section 5, BallardFavero-Katzarkov [9, Theorem 5.2.1] showed that such a semiorthogonal decomposition exists (see also $[55,71]$ ). A semiorthogonal summand of $D^{b}\left(\mathfrak{X}_{+}\right)$gives a $K$-motive (see [52, Section 4]), which in turn defines a direct summand of the topological $K$-group of $\mathfrak{X}_{+}{ }^{24}$. We expect that (8.3) arises from (8.4) in this way (cf. [76] for the discussion on Hochschild homology). On the other hand, in view of the deformation invariance of Gromov-Witten invariants, it is more natural to state conjectures in terms of topological $K$-groups instead of derived categories.

\subsection{Functoriality and Riemann-Hilbert problem}

We discuss how to recover the quantum cohomology of $\mathfrak{X}_{-}$from the quantum cohomology of $\mathfrak{X}_{+}$, assuming Conjecture 8.3. This involves solving a Riemann-Hilbert boundary value problem.

Proposition 8.5. Assume that the quantum D-modules $\mathrm{QDM}^{\mathrm{an}}\left(\mathfrak{X}_{ \pm}\right)$are of exponential type. Suppose that we are given the analytic continuation of the quantum D-module $\mathrm{QDM}^{\mathrm{an}}\left(\mathfrak{X}_{+}\right)$to a neighbourhood $W$ of $\tau_{+} \in H_{\mathrm{CR}}^{*}\left(\mathfrak{X}_{+}\right)$and its formal decomposition

$$
\left.\overline{\operatorname{QDM}^{\mathrm{an}}}\left(\mathfrak{X}_{+}\right)\right|_{W}=\mathscr{Q} \oplus \mathscr{R}
$$

corresponding to the decomposition (8.1) for which Conjecture 8.3 holds. Then we can recover the map $f$ in Conjecture 8.3 and the quantum D-module $f^{*} \mathrm{QDM}^{\text {an }}\left(\mathfrak{X}_{-}\right)$of $\mathfrak{X}_{-}$(trivialized as a vector bundle over $W \times \mathbb{C})$ together with an isomorphism $\left.f^{*} \overline{\mathrm{QDM}^{\mathrm{an}}\left(\mathfrak{X}_{-}\right)}\right|_{W} \cong \mathscr{Q}$.

Before giving a proof of this proposition, we review the exponential type assumption (see [70, Definition 2.12]). This was originally introduced by Hertling-Sevenheck [57, Definition 8.1] under the name "require no ramification". We also review a mutation system of Sanda-Shamoto [101, Definition 2.30].

The quantum D-module of $\mathfrak{X}=\mathfrak{X}_{ \pm}$is of exponential type if for each $\tau \in H_{\mathrm{CR}}^{*}(\mathfrak{X})$, we have a formal decomposition of the quantum D-module $\mathrm{QDM}^{\mathrm{an}}(\mathfrak{X})_{\tau}:=\left.\mathrm{QDM}^{\mathrm{an}}(\mathfrak{X})\right|_{\{\tau\} \times \mathbb{C}}$ (see $[57$, Lemma 8.2]):

$$
\overline{\operatorname{QDM}^{\mathrm{an}}}(\mathfrak{X})_{\tau} \cong \bigoplus_{\mathbf{u} \in \operatorname{Spec}\left(E \star_{\tau}\right)}\left(e^{\mathbf{u} / z} \otimes \mathcal{F}_{\mathbf{u}}\right) \otimes_{\mathbb{C}\{z\}} \mathbb{C} \llbracket z \rrbracket,
$$

where $\operatorname{Spec}\left(E \star_{\tau}\right)$ denotes the set of (mutually distinct) eigenvalues of $E \star_{\tau}, e^{\mathbf{u} / z}$ denotes the rank one connection $(\mathbb{C}\{z\}, d+d(\mathbf{u} / z))$ and $\mathcal{F}_{\mathbf{u}}$ is a free $\mathbb{C}\{z\}$-module equipped with a regular singular meromorphic connection. The decomposition is (automatically) orthogonal with respect to the pairing $P$, and induces a $z$-sesquilinear pairing $P_{\mathbf{u}}$ on each $\mathcal{F}_{\mathbf{u}} ; \mathcal{F}_{\mathbf{u}}$ is called the regular singular piece in [57, Lemma 8.2]. Moreover, the Hukuhara-Turrittin theorem (see [57, Lemma 8.3] in

\footnotetext{
${ }^{24}$ I thank Sergey Galkin for this remark and pointing to the reference [52].
} 
this context) implies that, for each phase $\phi$ admissible for $\operatorname{Spec}\left(E \star_{\tau}\right)$, the formal decomposition admits a unique analytic lift over the sector $I_{\phi}=\left\{\left(r, e^{\mathbf{i} \theta}\right) \in \widetilde{\mathbb{C}}:|\theta-\phi|<\frac{\pi}{2}+\epsilon\right\}$ (for some $\epsilon>0$ )

$$
\left.\left.\pi^{*}\left(\mathrm{QDM}^{\mathrm{an}}(\mathfrak{X})_{\tau}\right)\right|_{I_{\phi}} \cong \bigoplus_{\mathbf{u} \in \operatorname{Spec}\left(E \star_{\tau}\right)} \pi^{*}\left(e^{\mathbf{u} / z} \otimes \mathcal{F}_{\mathbf{u}}\right)\right|_{I_{\phi}},
$$

where $\pi: \widetilde{\mathbb{C}} \rightarrow \mathbb{C}$ is the oriented real blowup. In Section 6.1 , we discussed in details the special case of these decompositions where the quantum cohomology is semisimple.

The analytic germ at $z=0$ of the quantum D-module $\mathrm{QDM}^{\mathrm{an}}(\mathfrak{X})_{\tau}$ can be determined by the formal decomposition (8.5) and the Stokes data. Sanda-Shamoto [101, Definition 2.30] encoded the Stokes data in linear-algebraic data which they called a mutation system. This can be viewed as a generalization of a marked reflection system in Section 6.2. Let $V$ denote the space of flat sections of $\mathrm{QDM}^{\mathrm{an}}(\mathfrak{X})_{\tau}$ over the sector $I_{\phi}^{\times}=\left\{z \in \mathbb{C}^{\times}:|\arg z-\phi|<\frac{\pi}{2}+\epsilon\right\}$. We define a pairing $[\cdot, \cdot)$ on $V$ by (cf. Section 6.2)

$$
\left[s_{1}, s_{2}\right)=P\left(s_{1}\left(e^{-\pi \mathbf{i}} z\right), s_{2}(z)\right),
$$

where $s_{1}\left(e^{-\pi \mathbf{i}} z\right)$ denotes the analytic continuation of $s_{1}(z)$ along the path $[0, \pi] \ni \theta \mapsto e^{-\mathbf{i} \theta} z$. The mutation system (see [101, Proposition 2.5, Section 2.7]) for $\operatorname{QDM}^{\text {an }}(\mathfrak{X})_{\tau}$ associated with the admissible phase $\phi$ is given by the data ${ }^{25}$

- the tuple $(V,[\cdot, \cdot))$ of a vector space and a pairing;

- the decomposition $V \cong \underset{\mathbf{u} \in \operatorname{Spec}\left(E_{\star_{\tau}}\right)}{\bigoplus} V_{\mathbf{u}}$ induced by the analytic lift (8.6), where $V_{\mathbf{u}}$ is the space of flat sections of $e^{\mathbf{u} / z} \otimes \mathcal{F}_{\mathbf{u}}$ over the sector $I_{\phi}^{\times}$

satisfying the semiorthogonality:

$$
\left[v_{1}, v_{2}\right)=0 \quad \text { if } v_{1} \in V_{\mathbf{u}_{1}}, v_{2} \in V_{\mathbf{u}_{2}} \text { and } \Im\left(e^{-\mathbf{i} \phi} \mathbf{u}_{1}\right)<\Im\left(e^{-\mathbf{i} \phi} \mathbf{u}_{2}\right) .
$$

The pairing $[\cdot, \cdot)$ restricted to $V_{\mathbf{u}}$ is induced by the pairing $P_{\mathbf{u}}$ on $\mathcal{F}_{\mathbf{u}}$ [57, Lemma 8.4]. It was shown [101, Proposition 2.5, Section 2.5] that a mutation system is equivalent to Stokes data (or a Stokes filtered local system) equipped with a pairing. By the Riemann-Hilbert correspondence (see [101, Section 2.7] in this context), the formal structure (8.5) and the mutation system together recover the analytic germ at $z=0$ of $\operatorname{QDM}(\mathfrak{X})_{\tau}$.

Proof of Proposition 8.5. Fix $\tau \in W$. We may assume that $\phi$ in Conjecture 8.3 is admissible for the set $\operatorname{Spec}\left(E \star_{\tau}\right)$ of eigenvalues of $E \star_{\tau}$ on $H_{\mathrm{CR}}^{*}\left(\mathfrak{X}_{+}\right)$, by perturbing $\phi$ if necessary. Under the assumption that $\mathrm{QDM}^{\mathrm{an}}\left(\mathfrak{X}_{+}\right)_{\tau}$ is of exponential type, the summands $\mathscr{Q}, \mathscr{R}_{1}, \mathscr{R}_{2}$ of $\overline{\mathrm{QDM}^{\mathrm{an}}}\left(\mathfrak{X}_{+}\right)_{\tau}$ admit decompositions similar to (8.5). We write $\operatorname{Spec}(\mathscr{Q})\left(\operatorname{resp} \operatorname{Spec}\left(\mathscr{R}_{i}\right)\right)$ for the set of the eigenvalues of the operators $-\nabla_{z^{2} \partial_{z}}$ on $\left.\mathscr{Q}\right|_{z=0}$ (resp. on $\left.\mathscr{R}_{i}\right|_{z=0}$ ). By Conjecture 8.3(1), the sets $\operatorname{Spec}(\mathscr{Q}), \operatorname{Spec}\left(\mathscr{R}_{1}\right), \operatorname{Spec}\left(\mathscr{R}_{2}\right)$ are mutually distinct; therefore $\mathscr{Q}, \mathscr{R}_{1}, \mathscr{R}_{2}$ are partial sums of the right-hand side of the formal decomposition (8.5) for $\mathfrak{X}=\mathfrak{X}_{+}$. Writing $V \cong \underset{\mathbf{u} \in \operatorname{Spec}\left(E \star_{\tau}\right)}{\bigoplus} V_{\mathbf{u}}$ for the mutation system of $\mathrm{QDM}^{\mathrm{an}}\left(\mathfrak{X}_{+}\right)_{\tau}$, we can decompose $V$ as

$$
V \cong V_{\mathscr{R}_{1}} \oplus V_{\mathscr{Q}} \oplus V_{\mathscr{R}_{2}}
$$

$$
\text { with } \begin{aligned}
V_{\mathscr{Q}} & =\bigoplus_{\mathbf{u} \in \operatorname{Spec}(\mathscr{Q})} V_{\mathbf{u}}, V_{\mathscr{R}_{i}}=\bigoplus_{\mathbf{u} \in \operatorname{Spec}\left(\mathscr{R}_{i}\right)} V_{\mathbf{u}} \text {. By Conjecture 8.3(2), the decomposition } \\
V_{\mathscr{Q}} & =\bigoplus_{\mathbf{u} \in \operatorname{Spec}(\mathscr{Q})} V_{\mathbf{u}}
\end{aligned}
$$

\footnotetext{
${ }^{25}$ We omitted the data of a labelling $\tau$ in [101, Definition 2.30] since it can be recovered from the phase $\phi$.
} 
gives the mutation system for $\mathrm{QDM}^{\mathrm{an}}\left(\mathfrak{X}_{-}\right)_{f(\tau)}$. The $\widehat{\Gamma}$-integral structure identifies $(V,[\cdot, \cdot))$ with $\left(K\left(\mathfrak{X}_{+}\right) \otimes \mathbb{C}, \chi\right)$ and the decomposition $(8.7)$ is identified with that (8.3) of $K\left(\mathfrak{X}_{+}\right)$by Conjecture $8.3(3)$. Then we obtain an isomorphism

$$
\Phi: V_{\mathscr{Q}} \cong K\left(\mathfrak{X}_{-}\right) \otimes \mathbb{C} .
$$

Since the inclusion $K\left(\mathfrak{X}_{-}\right) \rightarrow K\left(\mathfrak{X}_{+}\right)$respects the Euler pairing, we see that the restriction of the pairing $[\cdot, \cdot)$ on $V$ to $V_{\mathscr{Q}}$ coincides with the pairing on $V_{\mathscr{Q}}$ as a mutation system for $\mathrm{QDM}^{\mathrm{an}}\left(\mathfrak{X}_{-}\right)_{f(\tau)}$. Therefore the mutation system for $\mathrm{QDM}^{\mathrm{an}}\left(\mathfrak{X}_{-}\right)_{f(\tau)}$ can be recovered as a summand of the mutation system for $\operatorname{QDM}^{\text {an }}\left(\mathfrak{X}_{+}\right)_{\tau}$. Thus we recover the analytic germ at $z=0$ of $\mathrm{QDM}^{\mathrm{an}}\left(\mathfrak{X}_{-}\right)_{f(\tau)}$ by the Riemann-Hilbert correspondence. We write $\mathscr{Q}^{\text {an }}$ for the germ so reconstructed.

We extend $\mathscr{Q}^{\text {an }}$ to the trivial vector bundle $\mathrm{QDM}^{\text {an }}\left(\mathfrak{X}_{-}\right)_{f(\tau)}$ over $\mathbb{C}_{z}$ and construct the fundamental solution $L(f(\tau), z)$ for $\mathfrak{X}_{-}$(see Section 2.4) and the map $f$. Consider the trivial bundle $Q^{(\infty)}:=H^{*}\left(\mathfrak{X}_{-}\right) \times\left(\mathbb{P}^{1} \backslash\{0\}\right) \rightarrow\left(\mathbb{P}^{1} \backslash\{0\}\right)$ equipped with the meromorphic connection

$$
\nabla_{z \partial_{z}}^{(\infty)}=z \frac{\partial}{\partial z}-\frac{c_{1}\left(\mathfrak{X}_{-}\right) \cup}{z}+\mu
$$

This has $z^{-\mu} z^{c_{1}(\mathfrak{X})}$ as a fundamental solution and the facts recalled in Section 2.4 imply that the quantum connection on $\{f(\tau)\} \times \mathbb{C}_{z}$ is gauge equivalent to $\nabla^{(\infty)}$ via the gauge transformation by $L(f(\tau), z)$ (which is regular and the identity at $z=\infty$ ). We glue this trivial bundle $Q^{(\infty)}$ with the germ $\mathscr{Q}^{\text {an }}$ of vector bundle at $z=0$ to get a vector bundle $\widehat{Q}$ over $\mathbb{P}^{1}$. The gluing is given by sending a flat section $s \in V_{\mathscr{Q}}$ over the sector $I_{\phi}^{\times}$to the flat section for $\nabla^{(\infty)}$ :

$$
(2 \pi)^{-n / 2} z^{-\mu} z^{c_{1}\left(\mathfrak{X}_{-}\right)}\left(\widehat{\Gamma}_{\mathfrak{X}_{-}} \cdot(2 \pi \mathbf{i})^{\operatorname{deg}_{0} / 2} \operatorname{inv}^{*} \widetilde{\operatorname{ch}}(\Phi(s))\right)
$$

with $n=\operatorname{dim} \mathfrak{X}_{-}$, where $\Phi$ is the isomorphism in (8.8). In view of the definition of the $\widehat{\Gamma}$-integral structure, this glued bundle $\widehat{Q}$ must be isomorphic to the trivial extension of $\mathrm{QDM}^{\text {an }}\left(\mathfrak{X}_{-}\right)_{f(\tau)}$ to $\mathbb{P}^{1}$ (with respect to the given trivialization). In particular, $\widehat{Q}$ is trivial, and the identification $\left.Q^{(\infty)}\right|_{\infty} \cong H_{\mathrm{CR}}^{*}\left(\mathfrak{X}_{-}\right)$at infinity induces a global trivialization $\widehat{Q} \cong H_{\mathrm{CR}}^{*}\left(\mathfrak{X}_{-}\right) \times \mathbb{P}^{1}$. The trivial bundle $\widehat{Q}$ equipped with a meromorphic connection gives the quantum D-module $\operatorname{QDM}^{\text {an }}\left(\mathfrak{X}_{-}\right)_{f(\tau)}$. Moreover, the isomorphism $\left.\widehat{Q}\right|_{\mathbb{P}^{1} \backslash\{0\}} \cong Q^{(\infty)}$ written in the respective trivializations gives the fundamental solution $L(f(\tau), z)$ as an $\operatorname{End}\left(H_{\mathrm{CR}}^{*}\left(\mathfrak{X}_{-}\right)\right)$-valued function. Varying $\tau$ in $W$, we recover the full quantum connection for $f^{*} \mathrm{QDM}^{\text {an }}\left(\mathfrak{X}_{-}\right)$from $L(f(\tau), z)$. We also recover $f(\tau)$ from the expansion

$$
L(f(\tau), z)^{-1} 1=1+\frac{f(\tau)}{z}+O\left(z^{-2}\right) .
$$

The proposition is proved.

Remark 8.6. Choosing fundamental solutions for regular singular pieces, we can formulate the above reconstruction in terms of a Riemann-Hilbert boundary value problem for the triple $\left(Y, Y^{-}, L\right)$ of fundamental solutions, where $Y$ is a fundamental solution on the sector $I_{\phi}^{\times}$with prescribed asymptotics, $Y^{-}$is a fundamental solution on the opposite sector $I_{\phi+\pi}^{\times}$with prescribed asymptotics and $L$ is the fundamental solution around $z=\infty$. In the semisimple case, this was explained in details by Dubrovin [40, Lecture 4] and the method there applies to the situation of our main Theorem 7.33. In the semisimple case, the formal structure (8.5) is determined only by the eigenvalues of $E \star_{\tau}$, and therefore the asymptotic basis (or, if any, the corresponding exceptional collection) reconstructs the quantum D-module. 
Remark 8.7. When a candidate formal decomposition $\overline{\mathrm{QDM}^{\mathrm{an}}}\left(\mathfrak{X}_{+}\right)_{\tau} \cong \mathscr{R}_{1} \oplus \mathscr{Q} \oplus \mathscr{R}_{2}$ and a decomposition (8.3) of the $K$-group (corresponding to the analytic lift via the $\widehat{\Gamma}$-integral structure) are given, what is non-trivial in the above reconstruction is the triviality of the glued bundle $\widehat{Q}$.

Remark 8.8. The fundamental solution $L(\tau, z)$ in Section 2.4 is called a calibration in the theory of Frobenius manifolds. A calibration of a Frobenius manifold is not unique in general and its ambiguity was discussed in [40, Lemma 4.1]. The above procedure recovers, not only (the germ of) the quantum cohomology Frobenius manifold of $\mathfrak{X}_{-}$(if $f$ is submersive), but also its calibration. ${ }^{26}$ Since the $\widehat{\Gamma}$-integral structure was normalized at the large radius limit point, it somehow 'remembers' the limit point.

Remark 8.9. Conjecture 8.3 is closely related to Dubrovin's conjecture [39, Conjecture 4.2.2] (or Gamma conjecture [44, Section 4.6] or Dubrovin-type conjecture [101, Definition 5.2]). It can be viewed as a relative version of these conjectures.

\section{A The Brieskorn module in the weak Fano case}

We discuss the coherence and the locally-freeness of the equivariant Brieskorn module $\operatorname{Bri}_{\mathbb{T}}(F)$ mirror to the small quantum cohomology of a weak-Fano smooth toric DM stack $\mathfrak{X}$, near the large radius limit point $0_{\boldsymbol{\Sigma}}$. We stated this in Proposition 6.15. We can describe the Brieskorn module as a certain variant of the GKZ system (see [27, Section 5.2]). The coherence of the relevant GKZ system near the large radius limit point has been discussed by the author [62, Proposition 4.4] (on a neighbourhood of $0_{\Sigma}$ with the logarithmic locus deleted), Reichelt-Sevenheck [91, Theorem 3.7] (on a neighbourhood of $0_{\boldsymbol{\Sigma}}$ for toric manifolds) and Mann-Reichelt [81, Theorem 4.10] (similarly for toric orbifolds). We adapt the argument of [81] to our Brieskorn module with minor modifications. Note that we impose only minimal assumptions on $\mathfrak{X}$ and $S$; we do not assume compactness of $\mathfrak{X}$ nor a generation condition for $S$. We also deal with the equivariant case. In this sense our result is slightly more general than [81].

Let $\mathfrak{X}=\mathfrak{X}_{\Sigma}$ be a semiprojective smooth toric DM stack from Section 3.1.1 which is weak Fano, i.e., $-K_{\mathfrak{X}}$ is nef. Let $\Delta \subset \mathbf{N}_{\mathbb{R}}$ denote the fan polytope, that is, the union of simplices spanned by $\{0\} \cup\{b \in R(\boldsymbol{\Sigma}): \bar{b} \in \sigma\}$ over all maximal cones $\sigma$ of $\boldsymbol{\Sigma}$. The weak Fano assumption implies that $\Delta$ is a convex polytope. We assume that the set $S$ (which is used to construct the LG mirror) is contained in the fan polytope:

$$
S \subset \mathbf{N} \cap \Delta .
$$

This means that the base space $\mathcal{M}$ of the LG model corresponds to the small quantum cohomology locus of $\mathfrak{X}$ in Section 6.3. The definition of the Brieskorn module on the affine chart $\operatorname{Spec} \mathbb{C}\left[\boldsymbol{\Lambda}(\boldsymbol{\Sigma})_{+}\right]$does not rely on Assumption 3.1 and we do not need this assumption in the following discussion.

The equivariant Brieskorn module $\operatorname{Bri}_{\mathbb{T}}(F)$ on the affine chart $\operatorname{Spec} \mathbb{C}\left[\boldsymbol{\Lambda}(\boldsymbol{\Sigma})_{+}\right]$is given by the module

$$
\mathrm{G}:=\mathbb{C}\left[\mathbb{O}(\boldsymbol{\Sigma})_{+}\right][z] .
$$

Recall from Section 4.1 that, by choosing a splitting $\mathbb{L}_{\mathbb{C}}^{\star} \rightarrow\left(\mathbb{C}^{S}\right)^{\star}, \xi \mapsto \hat{\xi}$ of $(3.4)$, G has the structure of a module over the ring of differential operators:

$$
\mathrm{D}:=R_{\mathbb{T}}\left[\boldsymbol{\Lambda}(\boldsymbol{\Sigma})_{+}\right][z]\left\langle z \xi q \frac{\partial}{\partial q}: \xi \in \mathbb{L}_{\mathbb{C}}^{\star}\right\rangle
$$

\footnotetext{
${ }^{26}$ I thank Vasily Golyshev for asking me a question which led me to this observation.
} 
where $\chi_{i} \in R_{\mathbb{T}}$ acts by $z x_{i} \frac{\partial}{\partial x_{i}}+x_{i} \frac{\partial F}{\partial x_{i}}$ and $z \xi q \frac{\partial}{\partial q}$ acts by $z \hat{\xi} u \frac{\partial}{\partial u}+\hat{\xi} u \frac{\partial F}{\partial u}$. For convenience, we choose co-ordinates $q_{1}, \ldots, q_{m} \in \mathbb{C}[\boldsymbol{\Lambda}(\boldsymbol{\Sigma})]$ corresponding to a $\mathbb{Z}$-basis of $\boldsymbol{\Lambda}(\boldsymbol{\Sigma})$ and write $\theta_{i}=q_{i} \frac{\partial}{\partial q_{i}} ;$ then we have

$$
\mathrm{D}=R_{\mathbb{T}}\left[\boldsymbol{\Lambda}(\boldsymbol{\Sigma})_{+}\right][z]\left\langle z \theta_{1}, \ldots, z \theta_{m}\right\rangle .
$$

Let $\mathscr{G}$ and $\mathscr{D}$ denote the sheaves on

$$
B:=\operatorname{Spec}\left(R_{\mathbb{T}}\left[\boldsymbol{\Lambda}(\boldsymbol{\Sigma})_{+}\right][z]\right)=\operatorname{Spec}\left(\mathbb{C}\left[\boldsymbol{\Lambda}(\boldsymbol{\Sigma})_{+}\right]\right) \times \operatorname{Lie} \mathbb{T} \times \mathbb{C}_{z}
$$

corresponding to $G$ and $D$ respectively. The main result in the appendix is the following.

Proposition A.1. Suppose, as above, that $\mathfrak{X}$ is a weak Fano semiprojective toric stack and that $S$ satisfies (A.1). There exists a Zariski open subset $U$ of $\operatorname{Spec}\left(\mathbb{C}\left[\boldsymbol{\Lambda}(\boldsymbol{\Sigma})_{+}\right]\right)$containing the large radius limit point $0_{\boldsymbol{\Sigma}}$ such that $\left.\mathscr{G}\right|_{U \times \operatorname{Lie}} \mathbb{T} \times \mathbb{C}_{z}$ is a locally free coherent $\mathcal{O}_{U \times \text { Lie }} \mathbb{T} \times \mathbb{C}_{z}$-module of rank $\operatorname{dim} H_{\mathrm{CR}}^{*}(\mathfrak{X})$.

\section{A.1 Generators and relations}

By Remark 3.17, the equivariant Brieskorn module $\mathrm{G}$ is generated by $w_{v}:=u^{\left(\Psi^{\Sigma}(\bar{v}), v\right)}$ with $v \in \mathbf{N} \cap \Pi$ as a $\mathbb{C}\left[\boldsymbol{\Lambda}(\boldsymbol{\Sigma})_{+}\right][z]$-module. Relations among these generators as a D-module are given as follows.

Lemma A.2. For $b \in S$, we write $e_{b}^{\star}=\widehat{D}_{b}+\chi(b)$ with $\chi(b) \in \mathbf{M}\left(\right.$ recall that $\left.D_{b}=D\left(e_{b}^{\star}\right) \in \mathbb{L}^{\star}\right)$. We have the following relation in $\mathrm{G}$ :

$$
\begin{aligned}
& \left(\prod_{b \in S} \prod_{c=0}^{\nu_{b}-1}\left(z D_{b} q \frac{\partial}{\partial q}+\chi(b)-\left(\Psi_{b}^{\Sigma}(v)+c\right) z\right)\right) w_{v} \\
& \quad=\left(\prod_{b \in S} u_{b}^{\nu_{b}}\right) w_{v}=q^{\Psi(v)+\sum_{b \in S} \nu_{b} e_{b}-\Psi\left(v+\sum_{b \in S} \nu_{b} b\right)} w_{v+\sum_{b \in S} \nu_{b} b}
\end{aligned}
$$

for every $\left(\nu_{b}\right)_{b \in S} \in\left(\mathbb{Z}_{\geq 0}\right)^{S}$ and $v \in \mathbf{N} \cap \Pi$.

Proof. By definition, $z D_{b} q \frac{\partial}{\partial q}+\chi(b)$ acts on $\mathrm{G}$ by $z u_{b} \frac{\partial}{\partial u_{b}}+u_{b}$. The first equality follows from this and $u_{b} \frac{\partial}{\partial u_{b}} w_{v}=\Psi_{b}(v) w_{v}$. The second equality is just by definition, see Section 3.5.

When $b \in R(\boldsymbol{\Sigma})$ and $v \in \mathbf{N} \cap \Pi$ lie in the same cone of $\Sigma$, we have a relation

$$
w_{v+b}=\left(z D_{b} q \frac{\partial}{\partial q}+\chi(b)-z \Psi_{b}^{\Sigma}(v)\right) w_{v}
$$

in $\mathrm{G}$ by Lemma A.2. From this we can see that $\mathrm{G}$ is generated by finitely many $w_{v}$ as a Dmodule. For example, the set $\left\{w_{v}: v \in \operatorname{Box}(\boldsymbol{\Sigma})\right\}$ generates G. By Lemma A.2, $w_{v}$ is annihilated by $P_{v, \lambda} \in \mathrm{D}$

$$
\begin{aligned}
P_{v, \lambda}:= & \prod_{b \in S: \lambda_{b}>0} \prod_{c=0}^{\lambda_{b}-1}\left(z D_{b} q \frac{\partial}{\partial q}+\chi(b)-\left(\Psi_{b}^{\boldsymbol{\Sigma}}(v)+c\right) z\right) \\
& -q^{\lambda} \prod_{b \in S: \lambda_{b}<0} \prod_{c=0}^{-\lambda_{b}-1}\left(z D_{b} q \frac{\partial}{\partial q}+\chi(b)-\left(\Psi_{b}^{\boldsymbol{\Sigma}}(v)+c\right) z\right)
\end{aligned}
$$

for any $\lambda \in \mathbb{L} \cap \widehat{\mathrm{NE}}\left(\mathfrak{X}_{\boldsymbol{\Sigma}}\right) \subset \mathbb{Z}^{S}$, cf. [27, Section 5.1]. 


\section{A.2 Characteristic variety and coherence}

Define an increasing filtration $\mathcal{F}_{l}(\mathscr{D})$ of $\mathscr{D}$ by the rank of differential operators, i.e., $\mathcal{F}_{l}(\mathscr{D})$ consists of differential operators of the form

$$
\sum_{k_{1}+\cdots+k_{m} \leq l} a_{k}(q, \chi, z)\left(z \theta_{1}\right)^{k_{1}} \cdots\left(z \theta_{m}\right)^{k_{m}}
$$

Choose generators $w_{v_{1}}, \ldots, w_{v_{k}}$ of $\mathrm{G}$ as a $\mathrm{D}$-module and introduce a filtration on $\mathscr{G}$ by

$$
\mathcal{F}_{l}(\mathscr{G}):=\sum_{i=1}^{k} \mathcal{F}_{l}(\mathscr{D}) w_{v_{i}} .
$$

An easy argument shows that if $\left.\operatorname{gr}_{\mathcal{F}}(\mathscr{G})\right|_{V}$ is finitely generated as an $\mathcal{O}_{V}$-module for an open set $V \subset B$, then $\left.\mathscr{G}\right|_{V}$ is also finitely generated as an $\mathcal{O}_{V}$-module. We shall show that $\operatorname{gr}_{\mathcal{F}}(\mathscr{G})$ is finitely generated on a neighbourhood of $\left\{0_{\boldsymbol{\Sigma}}\right\} \times \operatorname{Lie} \mathbb{T} \times \mathbb{C}_{z}$.

The associated graded module $\operatorname{gr}_{\mathcal{F}}(\mathscr{G})$ is a $\operatorname{gr}_{\mathcal{F}}(\mathscr{D})$-module generated by $w_{v_{1}}, \ldots, w_{v_{k}}$. We have $\operatorname{gr}_{\mathcal{F}}(\mathscr{D})=\mathcal{O}_{B}\left[\xi_{1}, \ldots, \xi_{m}\right]$, where $\xi_{i}$ denotes the image of $z \theta_{i} \in \mathcal{F}_{1}(\mathscr{D})$. The characteristic variety $\operatorname{Ch}(\mathscr{G})$ of $\mathscr{G}$ is defined to be the support of $\operatorname{gr}_{\mathcal{F}}(\mathscr{G})$ as an $\mathcal{O}_{B}\left[\xi_{1}, \ldots, \xi_{m}\right]$-module. It is a closed subset of $B \times \mathbb{C}^{m}$ invariant under the dilation $\left(\xi_{1}, \ldots, \xi_{m}\right) \mapsto\left(\lambda \xi_{1}, \ldots, \lambda \xi_{m}\right)$ with $\lambda \in \mathbb{C}^{\times}$. Then $\left.\operatorname{gr}_{\mathcal{F}}(\mathscr{G})\right|_{V}$ is a finitely generated $\mathcal{O}_{V}$-module over the following set $V$ :

$$
V=\{x \in B:(x, \xi) \in \operatorname{Ch}(\mathscr{G}) \Longrightarrow \xi=0\} .
$$

Note that $\operatorname{Ch}(\mathscr{G})$ induces a closed subset $C \subset B \times \mathbb{P}^{m-1}$ and $V$ is the complement of $\pi(C)$, where $\pi: B \times \mathbb{P}^{m-1} \rightarrow B$ is the projection; thus $V$ is Zariski-open.

For a differential operator $a \in \mathcal{F}_{l}(\mathscr{D}) \backslash \mathcal{F}_{l-1}(\mathscr{D})$, its principal symbol $\sigma(a)$ is the image of $a$ in $\operatorname{gr}_{\mathcal{F}}(\mathscr{D})$; explicitly

$$
\sigma(a):=\sum_{k_{1}+\cdots+k_{m}=l} a_{k}(q, \chi, z) \xi_{1}^{k_{1}} \cdots \xi_{m}^{k_{m}} \quad \text { if } a=\sum_{k_{1}+\cdots+k_{m} \leq l} a_{k}(q, \chi, z)\left(z \theta_{1}\right)^{k_{1}} \cdots\left(z \theta_{m}\right)^{k_{m}} .
$$

The principal symbol of the relation $P_{v, \lambda}$ in (A.2) is given by

$$
\sigma\left(P_{v, \lambda}\right)= \begin{cases}\prod_{b \in S: \lambda_{b}>0} D_{b}(\xi)^{\lambda_{b}}-q^{\lambda} \prod_{b \in S: \lambda_{b}<0} D_{b}(\xi)^{-\lambda_{b}} & \text { if } \sum_{b \in S} \lambda_{b}=0, \\ \prod_{b \in S: \lambda_{b}>0} D_{b}(\xi)^{\lambda_{b}} & \text { if } \sum_{b \in S} \lambda_{b}>0, \\ -q^{\lambda} \prod_{b \in S: \lambda_{b}<0} D_{b}(\xi)^{-\lambda_{b}} & \text { if } \sum_{b \in S} \lambda_{b}<0,\end{cases}
$$

where $D_{b}(\xi):=\sigma\left(z D_{b} q \frac{\partial}{\partial q}\right)$ is a linear form in $\xi_{1}, \ldots, \xi_{m}$. Because $\sigma\left(P_{v, \lambda}\right)$ is independent of $v$, it is an annihilator of $\operatorname{gr}_{\mathcal{F}}(\mathscr{G})$. Therefore $\mathrm{Ch}(\mathscr{G})$ is contained in the closed subset of $B \times \mathbb{C}^{m}$ defined by $\sigma\left(P_{v, \lambda}\right)=0$ for all $\lambda \in \mathbb{L} \cap \widehat{\mathrm{NE}}(\mathfrak{X})$.

Lemma A.3. There exists a Zariski-open subset $U$ of $\operatorname{Spec} \mathbb{C}\left[\boldsymbol{\Lambda}(\boldsymbol{\Sigma})_{+}\right]$containing $0_{\boldsymbol{\Sigma}}$ such that $U \times \operatorname{Lie} \mathbb{T} \times \mathbb{C}_{z}$ is contained in the locus $V$ in (A.3).

Proof. Note that $\sigma\left(P_{v, \lambda}\right)$ does not depend on $(\chi, z) \in \operatorname{Lie} \mathbb{T} \times \mathbb{C}_{z}$. Therefore it suffices to show that $\xi=0$ if $\xi \in \mathbb{C}^{m}$ satisfies

$$
\left.\sigma\left(P_{v, \lambda}\right)\right|_{q=0 \Sigma}=0 \quad \text { for all } \lambda \in \mathbb{L} \cap \widehat{\mathrm{NE}}(\mathfrak{X}) .
$$

Suppose that $\xi \in \mathbb{C}^{m}$ satisfies (A.4). We first show that there exists a cone $\sigma \in \Sigma$ such that $\left\{b \in S: D_{b}(\xi) \neq 0\right\}=R(\boldsymbol{\Sigma}) \cap \sigma$. Let $\left\{b_{1}, \ldots, b_{s}\right\}$ be the set of $b \in S$ such that $D_{b}(\xi) \neq 0$. The 
relative interior of the convex hull of $\left\{b_{1}, \ldots, b_{s}\right\}$ intersects with the relative interior of some cone $\sigma$ of $\Sigma$. Hence we get a relation of the form

$$
\sum_{i=1}^{s} c_{i} \bar{b}_{i}-\sum_{b \in R(\boldsymbol{\Sigma}) \cap \sigma} f_{b} \bar{b}=0
$$

for some $c_{i}>0$ satisfying $\sum_{i=1}^{s} c_{i}=1$ and some $f_{b}>0$. The convexity of $\Delta$ together with (A.1) implies that $\sum_{b \in R(\boldsymbol{\Sigma}) \cap \sigma} f_{b} \leq 1$. We may further assume that $c_{i}$ and $f_{b}$ are rational numbers. Then for some positive integer $l$,

$$
\lambda:=\sum_{i=1}^{s} l c_{i} e_{b_{i}}-\sum_{b \in R(\boldsymbol{\Sigma}) \cap \sigma} l f_{b} e_{b} \in \mathbb{Z}^{S}
$$

belong to $\mathbb{L} \cap \widehat{\mathrm{NE}}(\mathfrak{X})$ (recall the definition of $\widehat{\mathrm{NE}}(\mathfrak{X})$ around (3.14)). Note that

$$
\sum_{b \in S} \lambda_{b}=l\left(\sum_{i=1}^{s} c_{i}-\sum_{b \in R(\boldsymbol{\Sigma}) \cap \sigma} f_{b}\right) \geq 0 .
$$

Therefore, if $\lambda \neq 0$, we have the relation

$$
0=\left.\sigma\left(P_{v, \lambda}\right)\right|_{q=0_{\Sigma}}=\prod_{i=1}^{s} D_{b_{i}}(\xi)^{l c_{i}} .
$$

This contradicts the fact that $D_{b_{1}}(\xi) \neq 0, \ldots, D_{b_{s}}(\xi) \neq 0$. Hence $\lambda=0$ and we conclude that $\left\{b_{1}, \ldots, b_{s}\right\}=R(\boldsymbol{\Sigma}) \cap \sigma$.

Now we have $D_{b}(\xi)=0$ for all $b \notin R(\boldsymbol{\Sigma}) \cap \sigma$. Since $\left\{D_{b} \in \mathbb{L}_{\mathbb{C}}^{\star}: b \notin R(\Sigma) \cap \sigma\right\}$ spans $\mathbb{L}_{\mathbb{C}}^{\star}$, we have $\xi=0$. The lemma is proved.

Corollary A.4. There exists a Zariski-open neighbourhood $U$ of $0_{\boldsymbol{\Sigma}}$ in $\operatorname{Spec} \mathbb{C}\left[\boldsymbol{\Lambda}(\boldsymbol{\Sigma})_{+}\right]$such that

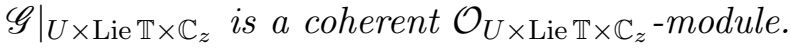

\section{A.3 Locally freeness and rank}

We complete the proof of Proposition A.1. Recall from Theorem 4.8 that the completed equivariant Brieskorn module is isomorphic to the quantum D-module of $\mathfrak{X}$. Thus we have

$$
\mathrm{G} / \mathfrak{m}_{\Sigma} \mathrm{G} \cong \widehat{\mathrm{G}} / \mathfrak{m}_{\Sigma} \widehat{\mathrm{G}} \cong H_{\mathrm{CR}, \mathbb{T}}^{*}(\mathfrak{X})[z],
$$

where $\mathfrak{m}_{\boldsymbol{\Sigma}} \subset \mathbb{C}\left[\boldsymbol{\Lambda}(\boldsymbol{\Sigma})_{+}\right]$denotes the maximal ideal corresponding to $0_{\boldsymbol{\Sigma}}$ and $\widehat{\mathrm{G}}$ denotes the $\mathfrak{m}_{\boldsymbol{\Sigma}^{-}}$ adic completion as discussed in Section 4.2. This implies that the restriction $\mathscr{G}_{\left\{0_{\boldsymbol{\Sigma}}\right\} \times \operatorname{Lie} \mathbb{T} \times \mathbb{C}_{z}}$ is free of rank $\operatorname{dim} H_{\mathrm{CR}}^{*}(\mathfrak{X})$. Hence by coherence, $\mathscr{G}$ is generated by $\operatorname{dim} H_{\mathrm{CR}}^{*}(\mathfrak{X})$ many sections in a neighbourhood of $\left\{0_{\boldsymbol{\Sigma}}\right\} \times \operatorname{Lie} \mathbb{T} \times \mathbb{C}_{z}$. On the other hand, the localization map "Loc" appearing in [27, Definition 4.17] gives $\operatorname{dim} H_{\mathrm{CR}}^{*}(\mathfrak{X})$ many linearly independent solutions of $\mathscr{G}$ for a generic $(\chi, z) \in \operatorname{Lie} \mathbb{T} \times \mathbb{C}_{z}$; the weak Fano condition ensures the convergence of the power series solution Loc. This implies that $\mathscr{G}$ is locally free of $\operatorname{rank} \operatorname{dim} H_{\mathrm{CR}}^{*}(\mathfrak{X})$ in a neighbourhood of $\left\{0_{\boldsymbol{\Sigma}}\right\} \times \operatorname{Lie} \mathbb{T} \times \mathbb{C}_{z}$.

To see that $\mathscr{G}$ is locally free on an open set of the form $U \times \operatorname{Lie} \mathbb{T} \times \mathbb{C}_{z}$ (for some open set $U \subset \operatorname{Spec} \mathbb{C}\left[\boldsymbol{\Lambda}(\boldsymbol{\Sigma})_{+}\right]$containing $\left.0_{\boldsymbol{\Sigma}}\right)$, we use the grading operator (4.1). The grading operator makes $\mathscr{G}$ a $\mathbb{C}^{\times}$-equivariant sheaf; the induced $\mathbb{C}^{\times}$-action on the base is given by the same grading operator on $\mathbb{C}\left[\boldsymbol{\Lambda}(\boldsymbol{\Sigma})_{+}\right][z]$ and the weight one $\mathbb{C}^{\times}$-action on Lie $\mathbb{T}$. 
Lemma A.5 (cf. [62, Section 3.1.4]). When $\mathfrak{X}$ is weak Fano, $\mathbb{C}\left[\mathbb{O}(\boldsymbol{\Sigma})_{+}\right]$is non-negatively graded with respect to the grading operator (4.1).

Proof. It suffices to show that $\sum_{b \in S} \lambda_{b} \geq 0$ for any $(\lambda, v) \in \mathbb{O}(\boldsymbol{\Sigma})_{+}$. By the definition (3.15) of $\mathbb{O}(\boldsymbol{\Sigma})_{+}$, it suffices to show that $\sum_{b \in S} \lambda_{b} \geq 0$ for all maximal cones $\sigma \in \Sigma$ and $\lambda \in \widetilde{C}_{\boldsymbol{\Sigma}, \sigma}$ (see (3.14)). Define a linear function $h: \mathbf{N}_{\mathbb{R}} \rightarrow \mathbb{R}$ by $h(\bar{b})=1$ for all $b \in R(\boldsymbol{\Sigma}) \cap \sigma$. Then the weak Fano condition (i.e., the convexity of $\Delta$ ) together with (A.1) implies that $h(\bar{b}) \leq 1$ for all $b \in S$. Hence, for $\lambda \in \widetilde{C}_{\boldsymbol{\Sigma}, \sigma}$, we have

$$
0 \leq h(\beta(\lambda))=\sum_{b \in R(\boldsymbol{\Sigma}) \cap \sigma} \lambda_{b}+\sum_{b \notin R(\boldsymbol{\Sigma}) \cap \sigma} \lambda_{b} h(\bar{b}) \leq \sum_{b \in S} \lambda_{b} .
$$

This proves the lemma.

In particular, $\mathbb{C}\left[\boldsymbol{\Lambda}(\boldsymbol{\Sigma})_{+}\right]$is non-negatively graded. Because the locus where $\mathscr{G}$ is locally free is preserved by the $\mathbb{C}^{\times}$-action and contains a neighbourhood of $\left(0_{\boldsymbol{\Sigma}}, 0,0\right) \in B$, it follows that $\mathscr{G}$ is locally free on an open set of the form $U \times \operatorname{Lie} \mathbb{T} \times \mathbb{C}_{z}$. The proof of Proposition A.1 is now complete.

Remark A.6. The generic rank of the GKZ system has been studied by many people, notably by Gelfand-Kapranov-Zelevinsky [46], Adolphson [6], Matusevich-Miller-Walther [83] and has been identified with the volume ${ }^{27}$ of $\Delta$ (when $\chi$ is not special). Over the open torus $\left(\mathbb{C}^{\times}\right)^{m}$ contained in Spec $\mathbb{C}\left[\boldsymbol{\Lambda}(\boldsymbol{\Sigma})_{+}\right]$, the Brieskorn module in this paper corresponds to the betterbehaved GKZ system of Borisov-Horja [17]; they showed that the generic rank of the betterbehaved GKZ system equals $\operatorname{vol}(\Delta)$ (independently of $\chi$ ).

\section{B Proof of Lemma 7.24}

We only prove Part (1) of the lemma; the argument for Part (2) is the same. It is easy to see that $F_{q, 0}^{-1}(\mathbf{u})$ and $\partial A_{q, 0}(\eta)$ intersect transversally at $x \in F_{q, 0}^{-1}(\mathbf{u}) \cap \partial A_{q, 0}(\eta)$ if and only if $\operatorname{grad} F_{q, 0}(x)$ and $\operatorname{grad} H(x)$ are linearly independent over $\mathbb{C}$, where we set

$$
\operatorname{grad} f(x)=\left(\overline{x_{i} \frac{\partial f}{\partial x_{i}}}\right)_{i=1}^{n} .
$$

Suppose that the lemma is not true. Then we can find sequences $(q(k), \mathbf{u}(k)) \in K$ and $x(k) \in$ $\mathcal{Y}_{q(k)}^{\mathrm{sm}}$ such that the following holds:

- $\eta_{k}=H(x(k)) \rightarrow \infty$ as $k \rightarrow \infty$;

- $F_{q(k), 0}(x(k))=\mathbf{u}(k)$;

- $\operatorname{grad} F_{q(k), 0}(x(k))$ and $\operatorname{grad} H(x(k))$ are linearly dependent over $\mathbb{C}$, i.e., there exists $\alpha_{k} \in \mathbb{C}$ such that $\operatorname{grad} F_{q(k), 0}(x(k))=\alpha_{k} \operatorname{grad} H(x(k))$.

Define an $\mathbb{R}^{n}$-valued function $\mathbf{v}(x)$ by

$$
\mathbf{v}(x)=\sum_{b \in S_{-}}\left|x^{b}\right|^{2} \bar{b}
$$

\footnotetext{
${ }^{27}$ The volume is normalized so that the standard simplex has volume 1 . When we allow $\mathbf{N}$ to have torsions, the generic rank is $\left|\mathbf{N}_{\text {tor }}\right| \times \operatorname{vol}(\Delta)$.
} 
Then we have

$$
\operatorname{grad} H(x)=\frac{\mathbf{v}(x)}{2 H(x)} .
$$

Writing $\mathbf{v} \cdot \mathbf{w}=\sum_{i=1}^{n} \mathbf{v}_{i} \mathbf{w}_{i}$ for the $\mathbb{C}$-bilinear scalar product, the third condition above can be written as

$$
\operatorname{grad} F_{q(k), 0}(x(k))=\frac{\operatorname{grad} F_{q(k), 0}(x(k)) \cdot \mathbf{v}(x(k))}{\|\mathbf{v}(x(k))\|^{2}} \mathbf{v}(x(k)) .
$$

By the curve selection lemma in [85, Lemma 2], we can find a real analytic curve $(0, \epsilon) \ni s \mapsto$ $(q(s), \mathbf{u}(s), x(s))$ admitting a Laurent expansion at $s=0$ such that for $0<s<\epsilon,(q(s), \mathbf{u}(s)) \in$ $K, F_{q(s), 0}(x(s))=\mathbf{u}(s)$,

$$
\operatorname{grad} F_{q(s), 0}(x(s))=\frac{\operatorname{grad} F_{q(s), 0}(x(s)) \cdot \mathbf{v}(x(s))}{\| \mathbf{v}\left(x(s) \|^{2}\right.} \mathbf{v}(x(s)),
$$

and $\lim _{s \rightarrow+0} H(x(s))=\infty$. Since $K$ is compact, $(q(0), \mathbf{u}(0)):=\lim _{s \rightarrow+0}(q(s), \mathbf{u}(s))$ exists in $K$. We write $x_{i}(s)=s^{\xi_{i}} a_{i}(1+O(s))$ with $a_{i} \neq 0$ and $\xi_{i} \in \mathbb{Z}$. Then the leading term for $H(x(s))^{2}$ is

$$
H(x(s))^{2}=\left(\sum_{b \in \sigma}|a|^{2 b}\right) s^{-2 m}+\text { higher order terms, }
$$

where we set $\sigma:=\left\{b \in S_{-}: \xi \cdot \bar{b}=-m\right\}$ with $\xi=\left(\xi_{1}, \ldots, \xi_{n}\right)$ and $-m:=\min \{\xi \cdot \bar{b}: b \in$ $\left.S_{-}\right\}$, and $|a|^{2 b}=\prod_{i=1}^{n}\left|a_{i}\right|^{2 b_{i}}$. Note that elements of $\sigma$ spans a face of the polytope $\Delta_{-}$. Since $\lim _{s \rightarrow+0} H(x(s))=\infty$, we have $m>0$. We calculate:

$$
\begin{aligned}
& \mathbf{v}(s)=\left(\sum_{b \in \sigma}|a|^{2 b} \bar{b}\right) s^{-2 m}+\text { higher order terms, } \\
& \mathbf{v}(s) \cdot \frac{d \log x(s)}{d s}=-m\left(\sum_{b \in \sigma}|a|^{2 b}\right) s^{-2 m-1}+\text { higher order terms, } \\
& \overline{\operatorname{grad} F_{q(s), 0}(x(s))}=\sum_{b \in S_{-}} q(s)^{\ell_{b}} x(s)^{b} \bar{b}=\left(\sum_{b \in \sigma} a^{b} q(0)^{\ell_{b}} \bar{b}\right) s^{-m}+\text { higher order terms. }
\end{aligned}
$$

By differentiating the equality $\mathbf{u}(s)=F_{q(s), 0}(x(s))$ in $s$, we get

$$
\begin{aligned}
& \frac{d u(s)}{d s}-\sum_{i=1}^{n} \frac{\partial F_{q(s), 0}(x(s))}{\partial q^{i}} \frac{d q^{i}(s)}{d s}=\overline{\operatorname{grad} F_{q(s), 0}(x(s))} \cdot \frac{d \log x(s)}{d s} \\
& =\left(\overline{\operatorname{grad} F_{q(s), 0}(x(s))} \cdot \frac{\mathbf{v}(x(s))}{\| \mathbf{v}\left(x(s) \|^{2}\right.}\right) \cdot\left(\mathbf{v}(x(s)) \cdot \frac{d \log x(s)}{d s}\right),
\end{aligned}
$$

where $\left\{q^{i}\right\}$ denotes a local co-ordinate system on $\mathcal{M}^{\mathrm{sm}, \times}$ and we used (B.1) in the second line. We compare the leading order terms as $s \rightarrow+0$. By the above calculation, the right-hand side of (B.3) has the leading term

$$
c\left(\sum_{b \in \sigma} a^{b} q(0)^{\ell} b \bar{b}\right) \cdot\left(\sum_{b \in \sigma}|a|^{2 b} \bar{b}\right) s^{-m-1}
$$


with $c=-m \sum_{b \in \sigma}|a|^{2 b} \neq 0$. On the other hand, because $q(s), \mathbf{u}(s)$ are regular at $s=0$ and

$$
\left|\frac{\partial F_{q, 0}(x(s))}{\partial q^{i}}\right|=\left|\sum_{b \in S} \frac{\partial q(s)^{\ell_{b}}}{\partial q^{i}} x^{b}\right| \leq C \cdot H(x(s)) \quad \text { for some } C>0,
$$

the left-hand side of (B.3) has poles of order at most $m$ by (B.2). Hence the quantity (B.4) must vanish. This together with (B.1) implies:

$$
\overline{\operatorname{grad} F_{q(s), 0}(x(s))}=\frac{\overline{\operatorname{grad} F_{q(s), 0}(x(s))} \cdot \mathbf{v}(x(s))}{\| \mathbf{v}\left(x(s) \|^{2}\right.} \mathbf{v}(x(s))=O\left(s^{-m+1}\right) .
$$

On the other hand, Proposition 7.19 and (B.2) give an estimate of the form

$$
\left\|\operatorname{grad} F_{q(s), 0}(x(s))\right\| \geq \epsilon_{1} H(x(s)) \geq \epsilon_{2} s^{-m}
$$

for sufficiently small $s>0$ (for some $\epsilon_{1}, \epsilon_{2}>0$ ). These two estimates contradict each other. Lemma 7.24 is proved.

\section{Acknowledgements}

I would like to thank Pedro Acosta, Arend Bayer, Andrea Brini, Tom Coates, Alessio Corti, Sergey Galkin, Vasily Golyshev, Eduardo González, Claus Hertling, Yuki Hirano, Paul Horja, Yunfeng Jiang, Yuan-Pin Lee, Chiu-Chu Melissa Liu, Wanmin Liu, Thomas Reichelt, Yongbin Ruan, Kyoji Saito, Fumihiko Sanda, Christian Sevenheck, Yota Shamoto, Mark Shoemaker, Takuro Mochizuki, Mauricio Romo, Hsian-Hua Tseng, Chris Woodward for many insightful discussions and explanations. I also thank the anonymous referees for their careful reading and helpful comments. This work is supported by EPSRC grant EP/E022162/1, and JSPS Kakenhi Grants Number 22740042, 23224002, 24224001, 25400069, 26610008, 16K05127, 16H06335, $16 \mathrm{H} 06337$ and 17H06127. Part of this work was done while I was in residence at the Mathematical Sciences Research Institute in Berkeley, California, during the Spring semester of 2018 and the stay was supported by the National Science Foundation under Grant No. DMS-1440140. I thank the organizers and participants of the programme for many stimulating discussions.

\section{References}

[1] Abramovich D., Graber T., Vistoli A., Algebraic orbifold quantum products, in Orbifolds in Mathematics and Physics (Madison, WI, 2001), Contemp. Math., Vol. 310, Amer. Math. Soc., Providence, RI, 2002, 1-24, arXiv:math.AG/0112004.

[2] Abramovich D., Graber T., Vistoli A., Gromov-Witten theory of Deligne-Mumford stacks, Amer. J. Math. 130 (2008), 1337-1398, arXiv:math.AG/0603151.

[3] Acosta P., Asymptotic expansion and the LG/(Fano, general type) correspondence, arXiv:1411.4162.

[4] Acosta P., Shoemaker M., Quantum cohomology of toric blowups and Landau-Ginzburg correspondences, Algebr. Geom. 5 (2018), 239-263, arXiv:1504.04396.

[5] Acosta P., Shoemaker M., Gromov-Witten theory of toric birational transformations, Int. Math. Res. Not., to appear, arXiv:1604.03491.

[6] Adolphson A., Hypergeometric functions and rings generated by monomials, Duke Math. J. 73 (1994), 269-290.

[7] Arnol'd V.I., Guseŭn-Zade S.M., Varchenko A.N., Singularities of differentiable maps, Vol. II, Monodromy and asymptotics of integrals, Monographs in Mathematics, Vol. 83, Birkhäuser Boston, Inc., Boston, MA, 1988.

[8] Ballard M., Diemer C., Favero D., Katzarkov L., Kerr G., The Mori program and non-Fano toric homological mirror symmetry, Trans. Amer. Math. Soc. 367 (2015), 8933-8974, arXiv:1302.0803. 
[9] Ballard M., Favero D., Katzarkov L., Variation of geometric invariant theory quotients and derived categories, J. Reine Angew. Math. 746 (2019), 235-303, arXiv:1203.6643.

[10] Balser W., Jurkat W.B., Lutz D.A., Birkhoff invariants and Stokes' multipliers for meromorphic linear differential equations, J. Math. Anal. Appl. 71 (1979), 48-94.

[11] Barannikov S., Semi-infinite Hodge structure and mirror symmetry for projective spaces, arXiv:math.AG/0010157.

[12] Bayer A., Semisimple quantum cohomology and blowups, Int. Math. Res. Not. 2004 (2004), 2069-2083, arXiv:math.AG/0403260.

[13] Bondal A., Orlov D., Semiorthogonal decomposition for algebraic varieties, arXiv:alg-geom/9506012.

[14] Borisov L.A., Chen L., Smith G.G., The orbifold Chow ring of toric Deligne-Mumford stacks, J. Amer. Math. Soc. 18 (2005), 193-215, arXiv:math.AG/0309229.

[15] Borisov L.A., Horja R.P., Mellin-Barnes integrals as Fourier-Mukai transforms, Adv. Math. 207 (2006), 876-927, arXiv:math.AG/0510486.

[16] Borisov L.A., Horja R.P., On the $K$-theory of smooth toric DM stacks, in Snowbird Lectures on String Geometry, Contemp. Math., Vol. 401, Amer. Math. Soc., Providence, RI, 2006, 21-42, arXiv:math.AG/0503277.

[17] Borisov L.A., Horja R.P., On the better behaved version of the GKZ hypergeometric system, Math. Ann. 357 (2013), 585-603, arXiv:1011.5720.

[18] Bridgeland T., Toledano Laredo V., Stokes factors and multilogarithms, J. Reine Angew. Math. 682 (2013), 89-128, arXiv:1006.4623.

[19] Brini A., Cavalieri R., Ross D., Crepant resolutions and open strings, J. Reine Angew. Math. 755 (2019), 191-245, arXiv:1309.4438.

[20] Bryan J., Graber T., The crepant resolution conjecture, in Algebraic Geometry - Seattle 2005, Part 1, Proc. Sympos. Pure Math., Vol. 80, Amer. Math. Soc., Providence, RI, 2009, 23-42, arXiv:math.AG/0610129.

[21] Cadman C., Using stacks to impose tangency conditions on curves, Amer. J. Math. 129 (2007), 405-427, arXiv:math.AG/0312349.

[22] Charest F., Woodward C.T., Floer cohomology and flips, arXiv:1508.01573.

[23] Chen W., Ruan Y., Orbifold Gromov-Witten theory, in Orbifolds in Mathematics and Physics (Madison, WI, 2001), Contemp. Math., Vol. 310, Amer. Math. Soc., Providence, RI, 2002, 25-85, arXiv:math.AG/0103156.

[24] Chen W., Ruan Y., A new cohomology theory of orbifold, Comm. Math. Phys. 248 (2004), 1-31, arXiv:math.AG/0004129.

[25] Clingempeel J., Le Floch B., Romo M., Brane transport in anomalous $(2,2)$ models and localization, arXiv:1811.12385.

[26] Coates T., Corti A., Iritani H., Tseng H.-H., A mirror theorem for toric stacks, Compos. Math. 151 (2015), 1878-1912, arXiv:1310.4163.

[27] Coates T., Corti A., Iritani H., Tseng H.-H., Hodge-theoretic mirror symmetry for toric stacks, J. Differential Geom. 114 (2020), 41-115, arXiv:1606.07254.

[28] Coates T., Givental A., Quantum Riemann-Roch, Lefschetz and Serre, Ann. of Math. 165 (2007), 15-53, arXiv:math.AG/0110142.

[29] Coates T., Iritani H., On the convergence of Gromov-Witten potentials and Givental's formula, Michigan Math. J. 64 (2015), 587-631, arXiv:1203.4193.

[30] Coates T., Iritani H., A Fock sheaf for Givental quantization, Kyoto J. Math. 58 (2018), 695-864, arXiv:1411.7039.

[31] Coates T., Iritani H., Jiang Y., The crepant transformation conjecture for toric complete intersections, Adv. Math. 329 (2018), 1002-1087, arXiv:1410.0024.

[32] Coates T., Iritani H., Tseng H.-H., Wall-crossings in toric Gromov-Witten theory. I. Crepant examples, Geom. Topol. 13 (2009), 2675-2744, arXiv:math.AG/0611550.

[33] Cotti G., Dubrovin B., Guzzetti D., Isomonodromy deformations at an irregular singularity with coalescing eigenvalues, Duke Math. J. 168 (2019), 967-1108, arXiv:1706.04808.

[34] Cox D.A., Little J.B., Schenck H.K., Toric varieties, Graduate Studies in Mathematics, Vol. 124, Amer. Math. Soc., Providence, RI, 2011. 
[35] Diemer C., Katzarkov L., Kerr G., Compactifications of spaces of Landau-Ginzburg models, Izv. Math. 77 (2013), 487-508, arXiv:1207.0042.

[36] Diemer C., Katzarkov L., Kerr G., Symplectomorphism group relations and degenerations of LandauGinzburg models, J. Eur. Math. Soc. 18 (2016), 2167-2271, arXiv:1204.2233.

[37] Douai A., Sabbah C., Gauss-Manin systems, Brieskorn lattices and Frobenius structures. I, Ann. Inst. Fourier (Grenoble) $5 \mathbf{3}$ (2003), 1055-1116, arXiv:math.AG/0211352.

[38] Douai A., Sabbah C., Gauss-Manin systems, Brieskorn lattices and Frobenius structures. II, in Frobenius Manifolds, Aspects Math., Vol. E36, Friedr. Vieweg, Wiesbaden, 2004, 1-18, arXiv:math.AG/0211353.

[39] Dubrovin B., Geometry and analytic theory of Frobenius manifolds, Doc. Math. (1998), extra Vol. II, 315-326, arXiv:math.AG/9807034.

[40] Dubrovin B., Painlevé transcendents in two-dimensional topological field theory, in The Painlevé Property, CRM Ser. Math. Phys., Springer, New York, 1999, 287-412, arXiv:math.AG/9803107.

[41] Fang B., Zhou P., Gamma II for toric varieties from integrals on T-dual branes and homological mirror symmetry, arXiv:1903.05300.

[42] Fantechi B., Mann E., Nironi F., Smooth toric Deligne-Mumford stacks, J. Reine Angew. Math. 648 (2010), 201-244, arXiv:0708.1254.

[43] Galkin S., The conifold point, arXiv:1404.7388.

[44] Galkin S., Golyshev V., Iritani H., Gamma classes and quantum cohomology of Fano manifolds: gamma conjectures, Duke Math. J. 165 (2016), 2005-2077, arXiv:1404.6407.

[45] Gel'fand I.M., Kapranov M.M., Zelevinsky A.V., Discriminants, resultants, and multidimensional determinants, Mathematics: Theory \& Applications, Birkhäuser Boston, Inc., Boston, MA, 1994.

[46] Gel'fand I.M., Zelevinskii A.V., Kapranov M.M., Hypergeometric functions and toric varieties, Funct. Anal. Appl. 23 (1989), 94-106.

[47] Givental A.B., Homological geometry and mirror symmetry, in Proceedings of the International Congress of Mathematicians, Vols. 1, 2 (Zürich, 1994), Birkhäuser, Basel, 1995, 472-480.

[48] Givental A.B., Elliptic Gromov-Witten invariants and the generalized mirror conjecture, in Integrable Systems and Algebraic Geometry (Kobe/Kyoto, 1997), World Sci. Publ., River Edge, NJ, 1998, 107-155, arXiv:math.AG/9803053.

[49] Givental A.B., Gromov-Witten invariants and quantization of quadratic Hamiltonians, Mosc. Math. J. 1 (2001), 551-568, arXiv:math.AG/0108100.

[50] Givental A.B., Semisimple Frobenius structures at higher genus, Int. Math. Res. Not. 2001 (2001), 12651286, arXiv:math.AG/0008067.

[51] González E., Woodward C.T., Quantum cohomology and toric minimal model programs, Adv. Math. 353 (2019), 591-646, arXiv:1207.3253.

[52] Gorchinskiy S., Orlov D., Geometric phantom categories, Publ. Math. Inst. Hautes Études Sci. 117 (2013), 329-349, arXiv:1209.6183.

[53] Graber T., Pandharipande R., Localization of virtual classes, Invent. Math. 135 (1999), 487-518, arXiv:alggeom/9708001.

[54] Gross M., Tropical geometry and mirror symmetry, CBMS Regional Conference Series in Mathematics, Vol. 114, Amer. Math. Soc., Providence, RI, 2011.

[55] Halpern-Leistner D., The derived category of a GIT quotient, J. Amer. Math. Soc. 28 (2015), 871-912, arXiv:1203.0276.

[56] Herbst M., Hori K., Page D., Phases of $\mathcal{N}=2$ theories in 1+1 dimensions with boundary, arXiv:0803.2045.

[57] Hertling C., Sevenheck C., Nilpotent orbits of a generalization of Hodge structures, J. Reine Angew. Math. 609 (2007), 23-80, arXiv:math.AG/0603564.

[58] Hori K., Vafa C., Mirror symmetry, arXiv:hep-th/0002222.

[59] Iritani H., Convergence of quantum cohomology by quantum Lefschetz, J. Reine Angew. Math. 610 (2007), 29-69, arXiv:math.DG/0506236.

[60] Iritani H., Fourier-Mukai transformation and toric quantum cohomology, Talk at Korean Institute for Advanced Study on June 10, 2008, as part of the Workshop on Gromov-Witten Theory and Related Topics, June 09-13, 2008, http://workshop.kias.re.kr/grw/. 
[61] Iritani H., Quantum D-modules and generalized mirror transformations, Topology 47 (2008), 225-276, arXiv:math.DG/0411111.

[62] Iritani H., An integral structure in quantum cohomology and mirror symmetry for toric orbifolds, Adv. Math. 222 (2009), 1016-1079, arXiv:0903.1463.

[63] Iritani H., Wall-crossings in toric Gromov-Witten theory III, Talk at Massachusetts Institute of Technology on June 25, 2009, as part of the Workshop on Mirror Symmetry and Related Topics, June 22-26, 2009, Notes and slides available at https://math. berkeley.edu/ auroux/frg/mit09-notes/.

[64] Iritani H., Quantum cohomology and periods, Ann. Inst. Fourier (Grenoble) 61 (2011), 2909-2958, arXiv:1101.4512.

[65] Iritani H., A mirror construction for the big equivariant quantum cohomology of toric manifolds, Math. Ann. 368 (2017), 279-316, arXiv:1503.02919.

[66] Iwanari I., The category of toric stacks, Compos. Math. 145 (2009), 718-746, arXiv:math.AG/0610548.

[67] Iwanari I., Logarithmic geometry, minimal free resolutions and toric algebraic stacks, Publ. Res. Inst. Math. Sci. 45 (2009), 1095-1140, arXiv:0707.2568.

[68] Jiang Y., The orbifold cohomology ring of simplicial toric stack bundles, Illinois J. Math. 52 (2008), 493-514, arXiv:math.AG/0504563.

[69] Jinzenji M., Coordinate change of Gauss-Manin system and generalized mirror transformation, Internat. J. Modern Phys. A 20 (2005), 2131-2156, arXiv:math.AG/0310212.

[70] Katzarkov L., Kontsevich M., Pantev T., Hodge theoretic aspects of mirror symmetry, in From Hodge theory to integrability and TQFT $t t^{*}$-geometry, Proc. Sympos. Pure Math., Vol. 78, Amer. Math. Soc., Providence, RI, 2008, 87-174, arXiv:0806.0107.

[71] Kawamata Y., Log crepant birational maps and derived categories, J. Math. Sci. Univ. Tokyo 12 (2005), 211-231, arXiv:math.AG/0311139.

[72] Kawamata Y., Derived categories and birational geometry, in Algebraic Geometry - Seattle 2005, Part 2, Proc. Sympos. Pure Math., Vol. 80, Amer. Math. Soc., Providence, RI, 2009, 655-665, arXiv:0804.3150.

[73] Kerr G., Weighted blowups and mirror symmetry for toric surfaces, Adv. Math. 219 (2008), 199-250, arXiv:math.AG/0609162.

[74] Kontsevich M., Birational invariants from quantum cohomology, Talk at Higher School of Economics on May 27, 2019, as part of Homological Mirror Symmetry at HSE, May 27 - June 1, 2019, https: //euro-math-soc.eu/event/mon-27-may-19-0000/homological-mirror-symmetry-hse.

[75] Kouchnirenko A.G., Polyèdres de Newton et nombres de Milnor, Invent. Math. 32 (1976), 1-31.

[76] Kuznetsov A., Hochschild homology and semiorthogonal decompositions, arXiv:0904.4330.

[77] Lee Y.-P., Lin H.-W., Wang C.-L., Flops, motives, and invariance of quantum rings, Ann. of Math. 172 (2010), 243-290, arXiv:math.AG/0608370.

[78] Lee Y.-P., Lin H.-W., Wang C.-L., Quantum cohomology under birational maps and transitions, in String-Math 2015, Proc. Sympos. Pure Math., Vol. 96, Amer. Math. Soc., Providence, RI, 2017, 149-168, arXiv:1705.04799.

[79] Lee Y.-P., Lin H.-W., Wang C.-L., Quantum flips I: local model, arXiv:1912.03012.

[80] Li A.-M., Ruan Y., Symplectic surgery and Gromov-Witten invariants of Calabi-Yau 3-folds, Invent. Math. 145 (2001), 151-218, arXiv:math.AG/9803036.

[81] Mann E., Reichelt T., Logarithmic degenerations of Landau-Ginzburg models for toric orbifolds and global $t t^{*}$ geometry, arXiv:1605.08937.

[82] Matsumura H., Commutative ring theory, 2nd ed., Cambridge Studies in Advanced Mathematics, Vol. 8, Cambridge University Press, Cambridge, 1989.

[83] Matusevich L.F., Miller E., Walther U., Homological methods for hypergeometric families, J. Amer. Math. Soc. 18 (2005), 919-941, arXiv:math.AG/0406383.

[84] Milnor J., Singular points of complex hypersurfaces, Annals of Mathematics Studies, Vol. 61, Princeton University Press, Princeton, N.J., University of Tokyo Press, Tokyo, 1968.

[85] Némethi A., Zaharia A., Milnor fibration at infinity, Indag. Math. (N.S.) 3 (1992), 323-335.

[86] Oda T., Park H.S., Linear Gale transforms and Gel'fand-Kapranov-Zelevinskij decompositions, Tohoku Math. J. 43 (1991), 375-399. 
[87] Orlov D.O., Projective bundles, monoidal transformations, and derived categories of coherent sheaves, Izv. Math. 41 (1993), 133-141.

[88] Pandharipande R., Pixton A., Zvonkine D., Relations on $\overline{\mathcal{M}}_{g, n}$ via 3-spin structures, J. Amer. Math. Soc. 28 (2015), 279-309, arXiv:1303.1043.

[89] Parusiński A., On the bifurcation set of complex polynomial with isolated singularities at infinity, Compositio Math. 97 (1995), 369-384.

[90] Pham F., La descente des cols par les onglets de Lefschetz, avec vues sur Gauss-Manin, Astérisque 130 (1985), 11-47.

[91] Reichelt T., Sevenheck C., Logarithmic Frobenius manifolds, hypergeometric systems and quantum $\mathcal{D}-$ modules, J. Algebraic Geom. 24 (2015), 201-281, arXiv:1010.2118.

[92] Ruan Y., The cohomology ring of crepant resolutions of orbifolds, in Gromov-Witten theory of spin curves and orbifolds, Contemp. Math., Vol. 403, Amer. Math. Soc., Providence, RI, 2006, 117-126, arXiv:math.AG/0108195.

[93] Sabbah C., Hypergeometric period for a tame polynomial, C. R. Acad. Sci. Paris Sér. I Math. 328 (1999), 603-608, A longer version published in Port. Math. (N.S.) 63 (2006), 173-226, arXiv:math.AG/9805077.

[94] Sabbah C., Polarizable twistor D-modules, Astérisque 300 (2005), vi+208 pages, arXiv:math.AG/0503038.

[95] Sabbah C., Isomonodromic deformations and Frobenius manifolds. An introduction, Universitext, SpringerVerlag London, Ltd., London, 2007.

[96] Saito K., The higher residue pairings $K_{F}^{(k)}$ for a family of hypersurface singular points, in Singularities, Part 2 (Arcata, Calif., 1981), Proc. Sympos. Pure Math., Vol. 40, Amer. Math. Soc., Providence, RI, 1983, 441-463.

[97] Saito K., Period mapping associated to a primitive form, Publ. Res. Inst. Math. Sci. 19 (1983), $1231-1264$.

[98] Saito M., On the structure of Brieskorn lattice, Ann. Inst. Fourier (Grenoble) 39 (1989), 27-72.

[99] Sanda F., Fukaya categories and blow-ups, Ph.D. Thesis, Nagoya University, 2015, available at https: //doi.org/10.15083/00008467.

[100] Sanda F., Computation of quantum cohomology from Fukaya categories, arXiv:1712.03924.

[101] Sanda F., Shamoto Y., An analogue of Dubrovin's conjecture, Ann. Inst. Fourier (Grenoble), to appear, arXiv:1705.05989.

[102] Shadrin S., BCOV theory via Givental group action on cohomological fields theories, Mosc. Math. J. 9 (2009), 411-429, arXiv:0810.0725.

[103] The Stacks Project Authors, Stacks Project, 2020, https://stacks.math.columbia.edu/.

[104] Teleman C., The structure of 2D semi-simple field theories, Invent. Math. 188 (2012), 525-588, arXiv:0712.0160.

[105] Tseng H.-H., Orbifold quantum Riemann-Roch, Lefschetz and Serre, Geom. Topol. 14 (2010), 1-81, arXiv:math.AG/0506111.

[106] Tyomkin I., Tropical geometry and correspondence theorems via toric stacks, Math. Ann. 353 (2012), 945-995, arXiv:1001.1554.

[107] Wasow W., Asymptotic expansions for ordinary differential equations, Pure and Applied Mathematics, Vol. 14, Interscience Publishers John Wiley \& Sons, Inc., New York - London - Sydney, 1965.

[108] Zariski O., Samuel P., Commutative algebra, Vol. II, Graduate Texts in Mathematics, Vol. 29, SpringerVerlag, New York - Heidelberg, 1975.

[109] Zong Z., Equivariant Gromov-Witten theory of GKM orbifolds, arXiv:1604.07270. 